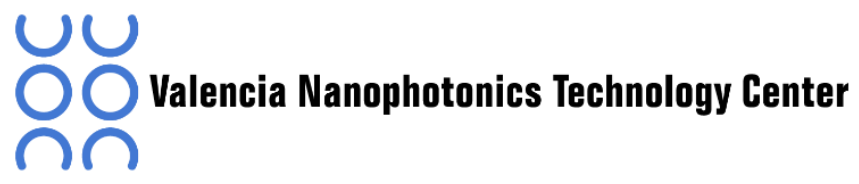

Thesis for the degree of Doctor of Philosophy

\title{
Advanced Signal Processing for Pulse-Amplitude Modulation Optical Transmission Systems
}

\section{Cristian Prodaniuc}

\author{
Supervisors: \\ Prof. Dr. Roberto Llorente Sáez \\ Dr. Nebojša Stojanović
}

January 2019 


\section{Acknowledgements}

The Ph.D. research has been a very interesting and exciting experience which helped me grow as an engineer and researcher. The lessons learned during this period will stay with me forever. While I have invested time and energy to complete this thesis, I wouldn't have gotten very far just on my own. In this opening section of the thesis I would like to give my thanks to all who have helped me reach my goals.

This Ph.D. Thesis would not have been possible without the support of Huawei German Research Center (GRC), who granted the access to state-of-the-art research facilities, and the Nanophotonics Technology Centre (NTC) of the Universitat Politècnica de València (UPV), who accepted and guided me as a doctorate student. I would like to thank especially my supervisors, Dr. Nebojša Stojanović from Huawei GRC and Prof. Dr. Roberto Llorente from the UPV; without their support I would not be where I am now. I will also like to thank my other colleagues from Huawei GRC for their friendship and advices.

I would like to dedicate this thesis and my work to those who were close to me during the last five years. To all my friends who helped me to be happy and to enjoy life, a big warm thank you. And to my mother a special thank you, as nothing would have been possible without you. 


\section{Abstract}

Non-coherent optical systems are currently employed in short-reach optical networks. These cover transmission distances ranging from 100 meters or less to up to $80 \mathrm{~km}$. The most common format currently found on the market is the four wavelength $(\lambda) 100 \mathrm{Gbps}(4 \lambda \times 25 \mathrm{Gbps})$ wavelength division multiplexing (WDM) transceiver. In recent years the non-coherent optical transmissions have been moving from $100 \mathrm{Gbps}$ to $400 \mathrm{Gbps}(100 \mathrm{Gbps} / \lambda)$. Since in the short-reach market the volume of optical devices being deployed is very large (at least in comparison with the long-haul market) the cost-per-unit of the devices is very important and it should be as low as possible. The $100 \mathrm{Gbps} / \lambda$ can be achieved by employing high bandwidth, high-performance components; however, this approach would significantly raise the cost of non-coherent optical transceivers, making this solution not feasible. Another approach is to use low-cost commercially available components and then improve performance by adding advanced signal processing (DSP) techniques to the system. The goal of this thesis is to investigate some of the most common DSP techniques used in non-coherent optical systems and also to investigate novel algorithms which could be applied to the scenario described above. In order for a DSP technique to be considered viable for the non-coherent WDM networks it has to effectively mitigate at least one of the three main impairments affecting such systems, those being the following: bandwidth limitations, chromatic dispersion (CD) and noise, be it optical noise generated by optical amplifiers or electrical noise generated by the electrical components.

A series of algorithms are proposed and examined in this thesis, and their performance is analyzed in simulations and also experimentally. For each algorithm we will have a look at how to generate/use the said algorithm and then break down the advantages and disadvantages of employing such an algorithm in the context of an optical transmission. Below a list detailing all the techniques that have been investigated in this thesis is given (in order of appearance):

- Feed-forward equalization (FFE): this is the most common equalizer and it is basically employed in every high-speed non-coherent optical transmission. It is easy to implement and can compensate for important bandwidth limitations. Its efficiency scales logarithmically (more or less) with its complexity.

- Maximum likelihood sequence estimation (MLSE): the MLSE is the optimum detector and thus provides the best performance when it comes to dealing with CD and bandwidth limitations. Its main drawback is its high complexity which scales exponentially with linear increases in $\mathrm{CD}$ or bandwidth limitations. 
- Geometrical constellation shaping: in multilevel intensity modulation schemes the distance between amplitude levels can be adjusted (such that they are no longer equidistant) in order to increase the signal's tolerance to noise

- Probabilistic shaping: another technique designed specifically for multilevel modulation schemes; it adjusts the probability of each amplitude level such that the tolerance to optical noise is increased

- Partial response signaling (PRS): it is a DSP approach where controlled inter-symbol interference (ISI) is intentionally introduced in such a way that the resulting signal requires less bandwidth. Its most common implementation is the duobinary (DB) signaling which, in tandem with the FFE, can significantly reduce the effects of bandwidth restriction in the transmission system. PRS can be customized to also mitigate $\mathrm{CD}$, effectively increasing transmission distances in non-coherent optical systems up to three times.

- Digital pre-emphasis (DPE): this technique consists in applying the inverse of the transfer function of the system to the signal at the transmitter side which reduces the impact of bandwidth limitations on the signal at the receiver side.

- Trellis-coded modulation (TCM): a modulation scheme that combines forward error correction (FEC) elements with set-partitioning techniques and multidimensional modulation to generate a signal that is more resistant to noise. On the downside this modulation scheme has increased sensitivity to ISI.

- Multidimensional set-partitioned modulation: very similar with TCM but without any FEC elements. It has lower gains than TCM in terms of noise tolerance but is less sensitive to ISI.

By using the techniques enumerated above it is possible to achieve $100 \mathrm{Gbps} / \lambda$ transmission speed when employing low-cost components. Even more, bit rates higher than $200 \mathrm{Gbps}$ are demonstrated in this thesis, showing that the non-coherent approach should still be viable also for the next generation of $800 \mathrm{Gbps}(4 \lambda \times 200 \mathrm{Gbps}) \mathrm{WDM}$ transceivers. 


\section{Resumen}

Los sistemas ópticos no-coherentes se emplean actualmente en redes ópticas de corto alcance y cubren distancias de transmisión que van desde 100 metros o menos hasta $80 \mathrm{~km}$. El formato más común que se encuentra actualmente en el estado del arte comercial se corresponde con sistemas con multiplexación por división de onda (WDM, wavelength division multiplexing) de cuatro longitudes de onda $(\lambda)$ con un régimen binario de $100 \mathrm{Gbps}(4 \lambda \times 25 \mathrm{Gbps})$. En los últimos años, las transmisiones ópticas no-coherentes están evolucionando de 100 Gbps a 400 Gbps (100 Gbps por $\lambda$ ). Dado que el mercado de sistemas ópticos de corto alcance comprende un volumen muy grande de unidades desplegadas (en comparación con el mercado de sistemas de transmisión a larga distancia), el coste por unidad es muy importante y debe ser lo más bajo posible. El régimen binario de $100 \mathrm{Gbps}$ / $\lambda$ pueden lograrse empleando componentes de gran ancho de banda y altas prestaciones; sin embargo, esto elevaría significativamente el coste de los transceptores ópticos no-coherentes, limitando la viabilidad comercial de esta solución. Otro enfoque más apropiado consiste en utilizar componentes disponibles comercialmente de relativamente bajo coste y, posteriormente, mejorar las prestaciones del sistema mediante procesado de señal digital (DSP, digital signal processing) o analógico en el sistema. El objetivo de esta tesis es investigar algunas de las técnicas de procesado más comunes utilizadas en sistemas ópticos no-coherentes y también investigar algoritmos nuevos que podrían aplicarse al escenario descrito anteriormente. Para que una técnica DSP sea considerada viable para la red WDM no-coherente, tiene que mitigar de manera efectiva al menos una de las tres limitaciones principales que afectan a dichos sistemas, que son las siguientes: limitaciones de ancho de banda, limitaciones por dispersión cromática $(\mathrm{CD}$, chromatic dispersion) y el ruido, considerando tanto el ruido óptico generado por los amplificadores ópticos como el ruido eléctrico generado por los componentes presentes en el sistema.

En esta tesis se proponen y examinan una serie de algoritmos, su rendimiento se analiza mediante simulación y también de manera experimental en laboratorio. Para cada algoritmo, se describe cómo generar/usar dicho algoritmo y a continuación se procede a evaluar sus ventajas e inconvenientes. A continuación, se proporciona una lista que detalla todas las técnicas que se investigan en esta tesis (en orden de aparición):

- Feed-forward equalizer (FFE): este es el ecualizador más común y se emplea básicamente en todas las transmisiones ópticas no coherentes de alta velocidad. Es fácil de implementar y puede compensar grandes limitaciones en el ancho de banda. Su eficiencia se escala logarítmicamente con su complejidad.

- Estimación de la secuencia de máxima verosimilitud (MLSE, maximum likelihood sequence estimation): el MLSE es el detector óptimo y, por lo tanto, proporciona las mejores prestaciones en detección cuando se abordan las limitaciones por CD y de ancho 
de banda. Su principal inconveniente es la alta complejidad, que crece exponencialmente con incrementos lineales frente a las limitaciones por CD o ancho de banda.

- Conformación de constelaciones geométricas: en los esquemas de modulación de intensidad multinivel, la distancia entre los niveles de amplitud se puede ajustar adecuadamente (de manera que no son equidistantes) a fin de aumentar la tolerancia frente a la relación señal a ruido.

- Conformación probabilística: otra técnica diseñada específicamente para esquemas de modulación multinivel. Esta técnica ajusta la probabilidad de cada nivel de amplitud de modo que se incremente la tolerancia al ruido óptico.

- Señalización de respuesta parcial (PRS, partial-response signalling): este es un enfoque DSP donde una interferencia entre símbolos controlada (ISI, inter-symbol interference) se introduce intencionalmente de tal manera que la señal resultante requiere menos ancho de banda. Su forma más común es la señalización duobinaria (DB), que en conjunto con la FFE puede reducir significativamente los efectos de las restricciones de ancho de banda en el sistema de transmisión. La técnica PRS se puede personalizar para combatir también el efecto de CD, lo que permite aumentar las distancias de transmisión en sistemas ópticos no coherentes hasta tres veces.

- Pre-énfasis digital (DPE, digital pre-emphasis): esta técnica consiste en aplicar el inverso de la función de transferencia del sistema a la señal en el lado del transmisor, lo que reduce el impacto de las limitaciones de ancho de banda en la señal en el lado del receptor.

- Modulación codificada Trellis (TCM, Trellis-coded modulation): esquema de modulación que combina elementos de corrección de errores (FEC, forward error correction) con técnicas de partición de conjuntos y modulación multidimensional para generar una señal más resistente al ruido. En el lado negativo, este esquema de modulación aumenta la sensibilidad al ISI.

Al utilizar las técnicas enumeradas anteriormente, es posible lograr un régimen binario de transmisión óptica de $100 \mathrm{Gbps} / \lambda$ empleando componentes de bajo coste. En esta tesis se demuestran incluso regímenes binarios de más de $200 \mathrm{Gbps}$, lo que indica que el enfoque nocoherente puede ser una solución viable para la próxima generación de transceptores ópticos WDM funcionado a $800 \mathrm{Gbps}(4 \lambda \times 200 \mathrm{Gbps})$. 


\section{Resum}

Actualment, s'utilitzen sistemes òptics no coherents en xarxes òptiques de curt abast. Estes xarxes cobreixen distàncies de transmissió que oscil·len entre 100 metres o menys fins a $80 \mathrm{~km}$. El format més comú que es troba actualment al mercat de sistemes de telecomunicació és el transceptor de multiplexació de divisió d'ona (WDM, wavelength division multiplexing) de quatre longituds d'ona $(\lambda)$ de $100 \mathrm{Gbps}(4 \lambda \times 25 \mathrm{Gbps})$. En els últims anys, les transmissions òptiques no coherents s'estan movent de $100 \mathrm{Gbps}$ a $400 \mathrm{Gbps}(100 \mathrm{Gbps} / \lambda)$. Atès que en el mercat de curt abast el volum de dispositius òptics que s'està desplegant és molt gran (almenys en comparació amb el mercat de llarg recorregut), el cost per unitat dels dispositius és molt important $i$ ha de ser el més baix possible. Els $100 \mathrm{Gbps} / \lambda$ es poden aconseguir mitjançant l'ús d'amples de banda elevats, components d'alta qualitat; no obstant això, aquest enfocament augmentaria significativament el cost dels transceptors òptics no coherents, cosa que fa que aquesta solució no sigui desitjable. Un altre enfocament és utilitzar components de baix cost comercialment disponibles i millorar el rendiment afegint processament de senyal digital (DSP, digital signal processing) al sistema. L'objectiu d'aquesta tesi és analitzar algunes de les tècniques DSP més habituals utilitzades en sistemes òptics no coherents i també investigar algorismes nous que es podrien aplicar a l'escenari descrit anteriorment. Per tal que una tècnica DSP es consideri viable per a la xarxa WDM no coherent, ha de mitigar efectivament almenys una de les tres principals limitacions que afecten aquests sistemes, que són les següents: limitacions d'ample de banda, dispersió cromàtica $(\mathrm{CD}$, chromatic dispersion) i soroll, ja sigui un soroll òptic generat per amplificadors òptics o sorolls elèctrics generats pels components elèctrics.

En aquesta tesi s'examina una sèrie d'algoritmes, el seu rendiment s'analitza en simulacions però també experimentalment. Per a cada algoritme, veurem com generar / utilitzar aquest algoritme i descompondre els avantatges i desavantatges d'utilitzar aquest algoritme. A continuació es mostra una llista que detalla totes les tècniques de que es presenten en aquesta tesi (en ordre d'aparició):

- Feed-forward equalizer (FFE): aquest és l'equalitzador més comú i s'utilitza bàsicament en totes les transmissions òptiques no coherents d'alta velocitat. És fàcil d'implementar i pot compensar grans quantitats de limitacions d'ample de banda. La seva eficiència escala de forma logarítmica amb la seva complexitat.

- Estimació de la seqüència de probabilitat màxima (MLSE, maximum likelihood sequence estimation): el MLSE és el detector òptim i, per tant, proporciona el millor rendiment quan es tracta de limitacions d'ample de banda i de CD. El seu principal inconvenient és la seva gran complexitat que augmenta exponencialment amb increments lineals en CD o en sistemes amb limitacions d'ample de banda. 
- Formació geomètrica de la constel-lació: en esquemes de modulació d'intensitat multinivell es pot ajustar la distància entre els nivells d'amplitud (de manera que ja no són equidistants) per augmentar la tolerància del senyal al soroll

- Confecció probabilística: una altra tècnica dissenyada específicament per als esquemes de modulació multinivell; ajusta la probabilitat de cada nivell d'amplitud de manera que augmenta la tolerància al soroll òptic

- Senyalització de resposta parcial (PRS, partial-response signalling): és un enfocament DSP on la interferència entre símbols controlats (ISI, inter-symbol interference) s'introdueix intencionalment de manera que el senyal resultant requereix menys ample de banda. La seva forma més comuna és la senyalització duobinària (DB), que juntament amb el FFE pot reduir significativament els efectes de les restriccions d'ample de banda en el sistema de transmissió. El PRS es pot personalitzar per combatre els efectes del CD, augmentant de manera efectiva les distàncies de transmissió en un sistema òptic no coherent fins a tres vegades.

- Pre-èmfasi digital (DPE, digital pre-emphasis): aquesta tècnica consisteix a aplicar la inversió de la funció de transferència del sistema a la senyal al costat del transmissor que redueix l'impacte de les limitacions d'ample de banda en la senyal al costat del receptor.

- Modulació codificada en enreixat (TCM, Trellis-coded modulation): esquema de modulació que combina els elements de correcció d'errors avançats (FEC, forward error correction) amb tècniques de partionament de conjunts i modulació multidimensional per generar un senyal més resistent al soroll. En contraprestació, aquest esquema de modulació augmenta la sensibilitat a l'ISI.

- Modulació multidimensional amb particions conjuntes: molt similar a TCM però sense elements FEC. Té guanys menors que TCM en termes de tolerància al soroll, però no és tan sensible a l'ISI.

Mitjançant l'ús de les tècniques enumerades anteriorment, és possible aconseguir un règim binari de transmissió òptica de $100 \mathrm{Gbps} / \lambda$ quan s'utilitzen components de baix cost. S'han demostrat règims binaris de més de $200 \mathrm{Gbps}$ en aquesta tesi, que indiquen que la tecnologia no-coherent és viable també per a la nova generació de sistemes transceptors òptics WDM funcionant a $800 \mathrm{Gbps}$ $(4 \lambda \times 200 \mathrm{Gbps})$. 


\section{Contents}

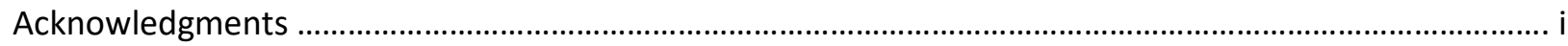

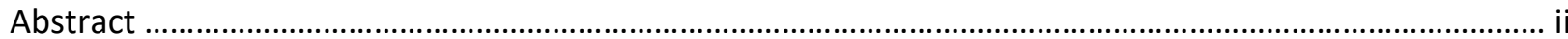

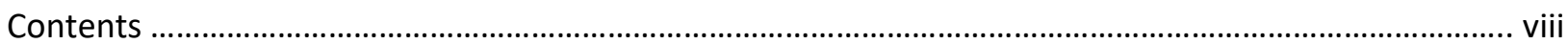

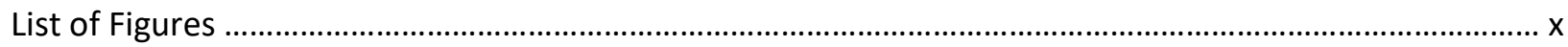

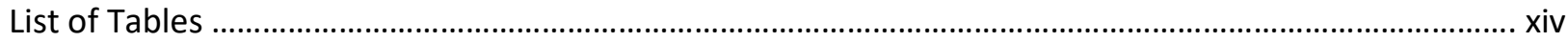

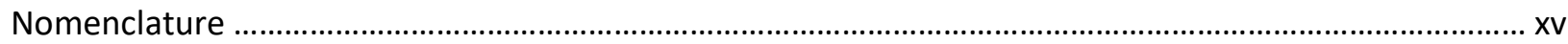

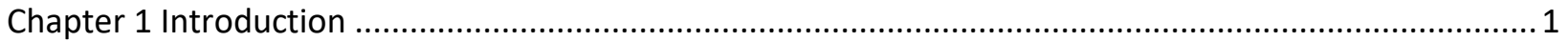

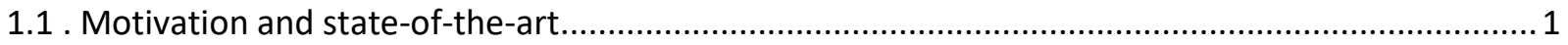

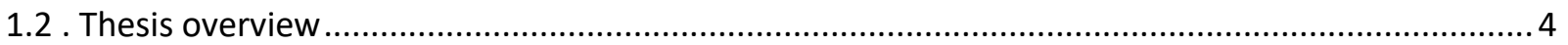

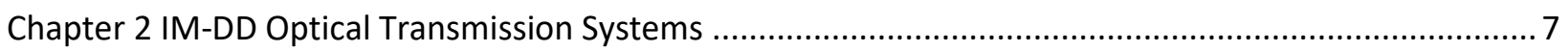

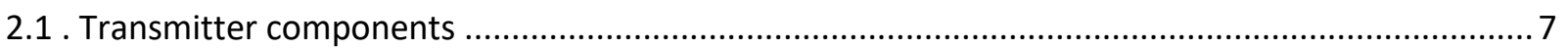

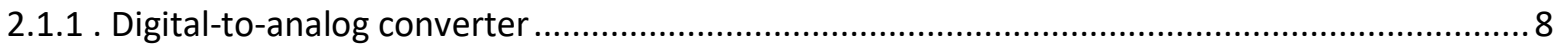

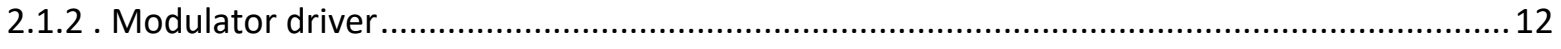

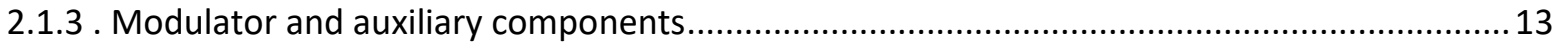

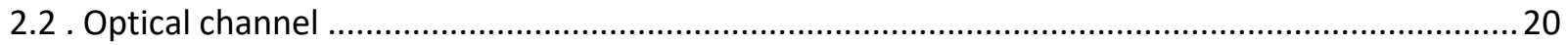

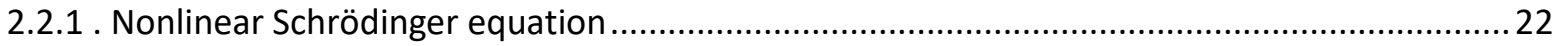

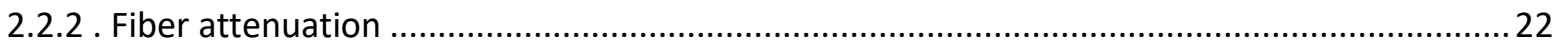

2.2.3 . Optical noise and the optical signal-to-noise ratio .............................................................23

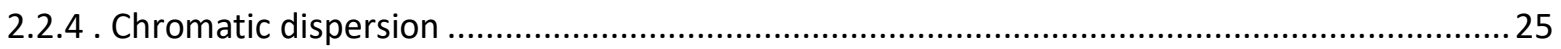

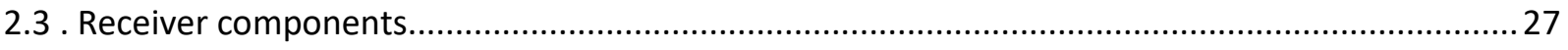

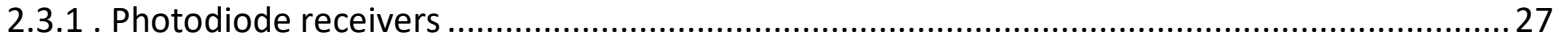

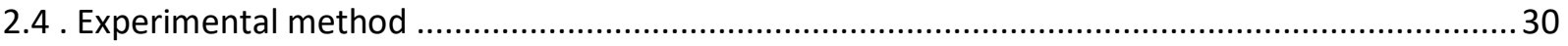

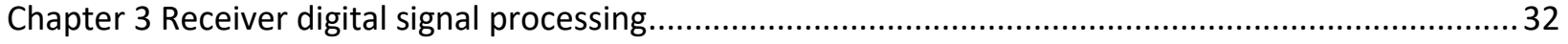

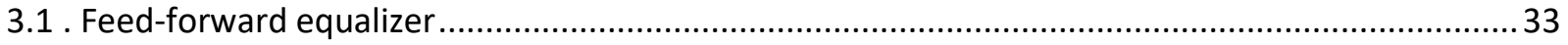

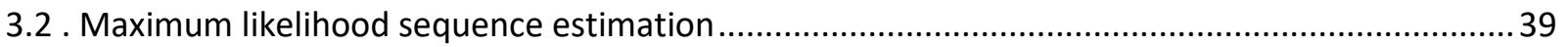

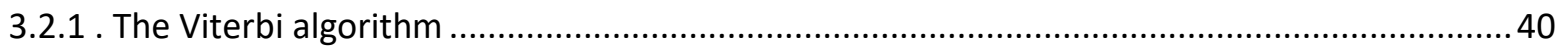

3.2.2. Simulation and experimental analysis of MLSE performance ............................................44

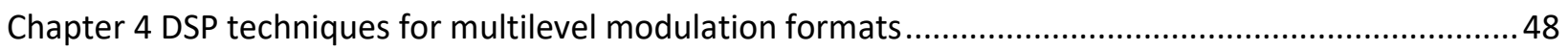

4.1. Multilevel intensity modulation generation ........................................................................... 48 


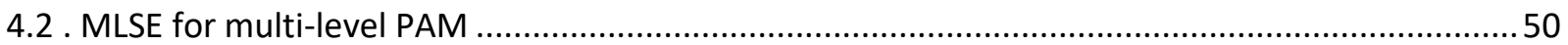

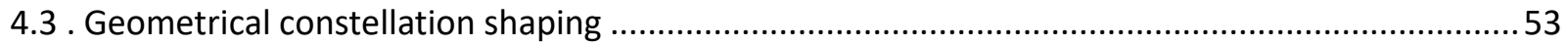

4.3.1. Geometrical shaping for mitigating the effects of noise .................................................5

4.3.2. Geometrical shaping for mitigating the effects of chromatic dispersion ..............................61

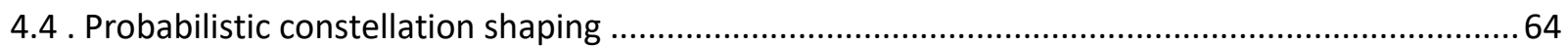

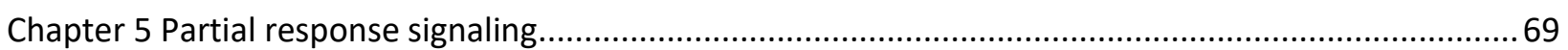

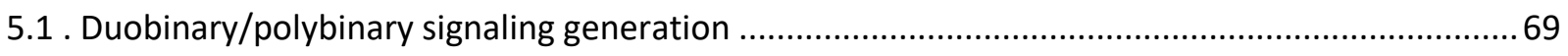

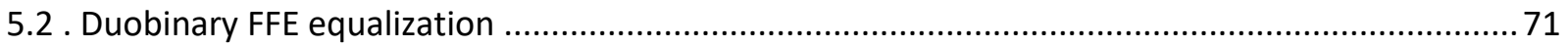

5.3 . Low-cost PAM solutions for $100 \mathrm{Gbps} /$ lambda transmissions ...................................................... 74

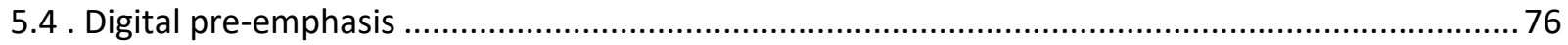

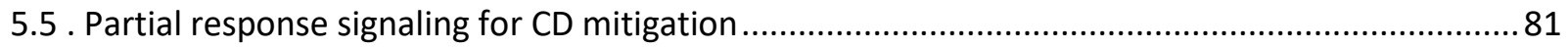

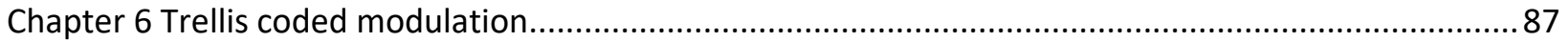

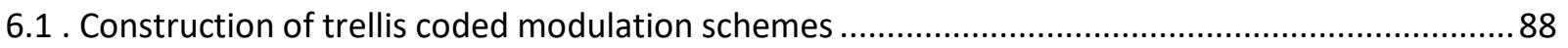

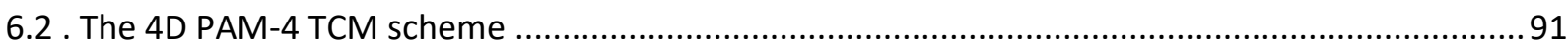

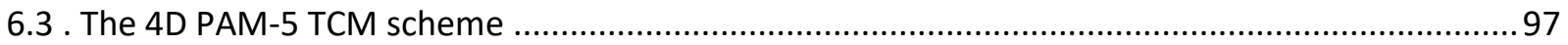

Chapter 7 Multidimensional multilevel set-partitioned modulation.................................................... 106

7.1. Generation of multidimensional set-partitioned modulation schemes ......................................107

7.2 . The 3D PAM-8 multidimensional set-partitioning modulation.................................................. 110

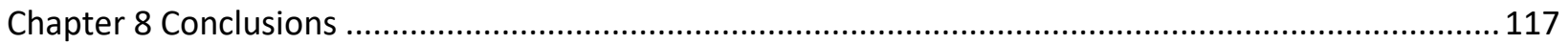

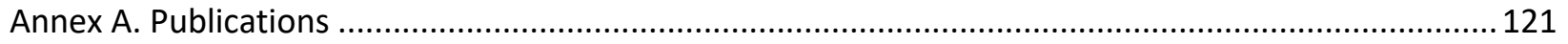

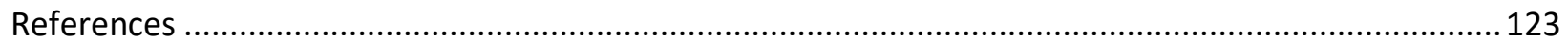




\section{List of figures}

Figure 1.1. Optical components sales (history and prediction - 2010 to 2023) [3] ................................. 2 Figure 1.2. Block diagram of a $100 / 400 \mathrm{Gbps} 4 \lambda$ WDM transceiver (EML - electro-absorption modulated laser, MUX - multiplexer, DMUX - demultiplexer, EDFA - erbium doped fiber amplifier, TR -timing

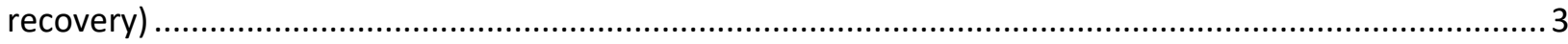
Figure 2.1. Block diagram of a basic optical transmission system (MD - modulator driver, EDFA - erbium

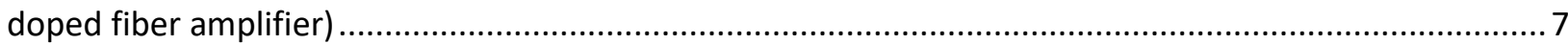

Figure 2.2 . DSP blocks of a basic communication transmitter.......................................................... 8 Figure 2.3. Eye diagrams at the output of the BPG (SHF 12301A): 28 GBaud OOK (left), 28 GBaud PAM-4

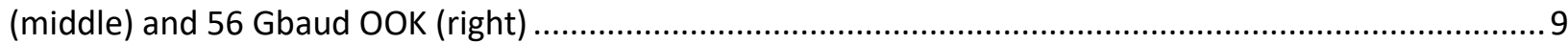

Figure 2.4. Generation of higher order modulations with the BPG................................................... 9 Figure 2.5. Fujitsu DAC transfer function (left) and the eye diagrams at the output of the Fujitsu DAC for

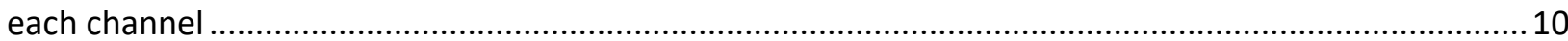

Figure 2.6. Spectrum comparison between two experimental setups, one using the DAC and the other the AWG (the rest of the components are identical) (left side) and the back-to-back (BTB), 28 GBaud PAM-4 eye diagrams after DAC/AWG and after receiver for the same setups (right side) ......................12

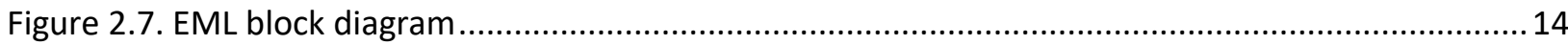

Figure 2.8. biasT circuit (a) and the bias versus output power curve of a commercially available $10 \mathrm{G}$

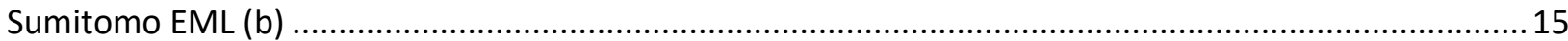

Figure 2.9. Basic block diagram of the transmitter side when employing a MZM ................................17

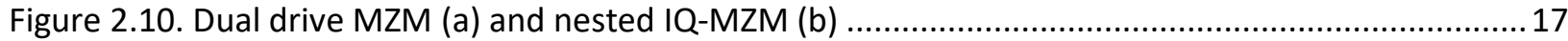

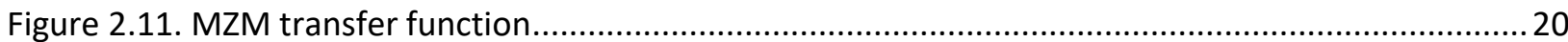

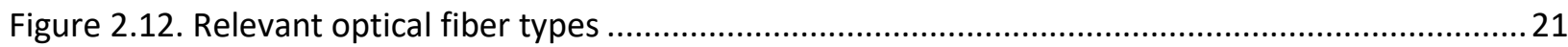

Figure 2.13. Fiber loss coefficient relative to wavelength for SSMF..................................................23

Figure 2.14. Optical transmission system employing EDFAs ..........................................................24

Figure 2.15. Illustration of OSNR measurement [45]. Noise power is measured on both sides of the signal

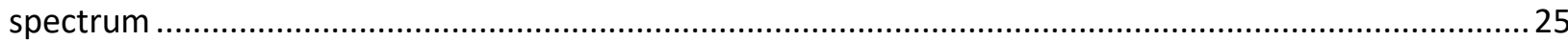

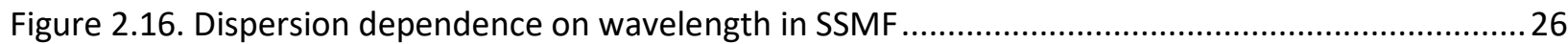

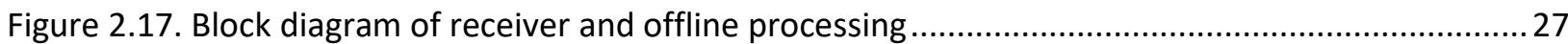

Figure 2.18. Performance of PAM-4 and DB PAM-4, 28 GBaud, BTB optical transmission illustrating the

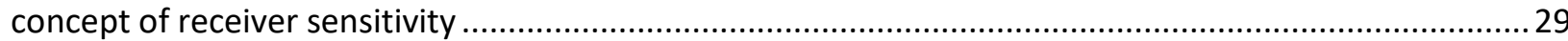

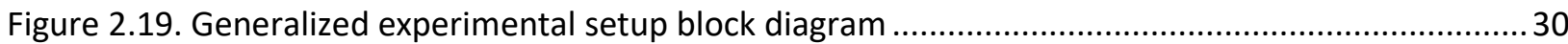

Figure 3.1. CFP optical module and the most commonly used DSP blocks ............................................32

Figure 3.2. Basic structure of a FFE filter (top) and a DFE filter (bottom) …...........................................33

Figure 3.3. The tap values for an 11-tap FIR filter for a 28 GBaud, PAM-4 BTB transmission (simulation). Pre-cursors are denoted with negative numbers and post-cursors with positive numbers. The bandwidths of the transmitter and of the receiver are varied to 25,18 and $10 \mathrm{GHz}$ .34 Figure 3.4. The influence of bandwidth limitations and reflections on the tap values of a 71-tap FFE for a high speed 90 GBaud PAM-4 BTB optical transmission

Figure 3.5. Initial tap value when the system transfer function is unknown (left) and for a specific known system (right). The values on the right side will always vary depending on the system 
Figure 3.6. The influence of $\mu$ on tap coefficients convergence for $\mu=0.01$ (a), $\mu=0.001$ (b), $\mu=0.0001$ (c)

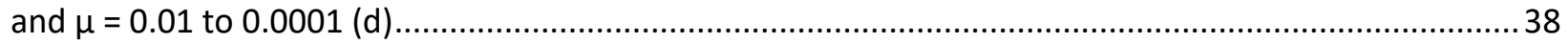

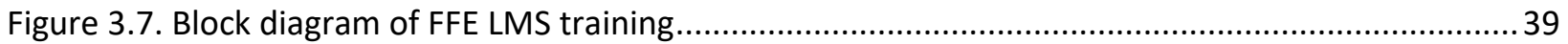

Figure 3.8. Tree diagram of Viterbi algorithm (a) and trellis diagram of the Viterbi algorithm (b)........... 40

Figure 3.9. Block diagram of a channel affected by ISI and AWGN .................................................. 42

Figure 3.10. Numeric example of the Viterbi algorithm (trellis diagram decoding) ................................. 43

Figure 3.11. Block diagram of 100G optical transmission system .....................................................44

Figure 3.12. Simulation comparison between one Sample/Symbol and two Sample/Symbols 16-state MLSE for a 28 GBaud, OOK, CD = $700 \mathrm{ps} / \mathrm{nm}$ transmission (a) and simulation of achievable transmission length versus required OSNR for a $28 \mathrm{GBaud}$, OOK system, with and without a 16-state MLSE..............45

Figure 3.13. Experimental setup for testing one samples/symbol and two samples/symbol MLSE performance

Figure 3.14. Experimental performance comparison between one sample/symbol and two sample/symbol MLSE for a 0, 40 and $80 \mathrm{~km}$ optical link (a) and received power sensitivity performance comparison (at -336, 336 and $1008 \mathrm{ps} / \mathrm{nm}$ ) between one sample/symbol and two sample/symbol MLSE

(b)

Figure 4.1. PAM-n generation

Figure 4.2. Transmission reach for $28 \mathrm{Gbps}$ OOK (red), PAM-4 (green) and PAM-8 (blue) when using no DSP or a 64-state MLSE (1-sample or 2-sample) - simulation results.

Figure 4.3. Experimental setup for testing MLSE performance in a 56 Gbps PAM-4 optical transmission 52 Figure 4.4. Simulation analysis on the performance of 16-state MLSE with CD of $170 \mathrm{ps} / \mathrm{nm}$ and $28 \mathrm{Gbps}$, PAM-4 (a) and BER variations in 56 Gbps, PAM-4 experiments after 0,4 , and 8 km links (b) .... .52 Figure 4.5. Equidistant levels PAM eye diagrams before DAC for PAM-4 (left), PAM-8 (middle) and PAM16 (right) .53

Figure 4.6. Transmission block diagram and signal notations ..........................................................54

Figure 4.7. Influence of AWGN on ESL PAM-4 (a) and on unequally spaced levels PAM-4 (b) .................55 Figure 4.8. BER in relation with second and third level amplitude for the following scenarios: $\sigma e l, T x=0.03, \sigma o p t=0, \sigma e l, R x=0.03$ (a), $\sigma e l, T x=0, \sigma o p t=0.06, \sigma e l, R x=0.015$ (b), $\sigma e l, T x=0.005, \sigma o p t=0.04, \sigma e l, R x=0.015$ (c), $\sigma e l, T x=0.015, \sigma o p t=0.03, \sigma e l, R x=0.015$ (d), $\sigma e l, T x=0.02, \sigma o p t=0.02, \sigma e l, R x=0.02$ (e), $\sigma e l, T x=0.03, \sigma o p t=0.015, \sigma e l, R x=0.015$ (f). The red dots indicate optimum levels from simulation and the cyan dots show the levels calculated with equation (62)

Figure 4.9. Experimental setup for testing the effects of BER performance of geometrical constellation shaping

Figure 4.10. Experimental performance of ESL, s-ESL and OSL PAM-4 for BTB (a) and of ESL and OSL for 5 and $10 \mathrm{~km}$ links. Baud rate $=28 \mathrm{GBaud}$ 61 Figure 4.11. Generation of optical PAM-4 with a BPG and IQ-MZM using just the I branch of the modulator (a) or using both the I and the $Q$ branches (b)

Figure 4.12. Optical eye diagram at the output of the MZM, when one branch is employed to generate PAM-4 (a) and when two branches are employed (b).....

Figure 4.13. The constellation of ESL PAM-4 (a) and of USL PAM-4 (b) and the corresponding eye

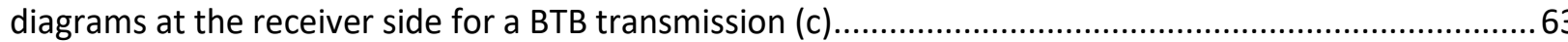
Figure 4.14. Experimental setup for testing USL PAM-4 CD mitigation capabilities 
Figure 4.15. Performance comparison between equally spaced levels (blue) and unequally spaced levels (magenta) PAM-4 (a), received eye diagrams for equally spaced levels (left column) and unequally spaced levels (right column) PAM-4 after 0, 5 and $10 \mathrm{~km}$ (b) and the unequally spaced levels PAM-4 constellation with corresponding optical power levels (at the receiver the levels will be inversed) (c) ...64 Figure 4.16. K vs. zero level probability for OOK (a) and for PAM-4 (b). Simulation of BER performance of OOK and PAM-4 with and without probabilistic shaping (c)

Figure 4.17. Experimental setup for testing the performance of probabilistic shaping.

Figure 4.18. OSNR performance comparison between classic PAM-4 and PAM-4 with probabilistic shaping for: BTB, with an 11-tap FFE (a) and $5 \mathrm{~km}$ with 11-tap FFE, 10 and $15 \mathrm{~km}$ with 11-tap FFE plus 64state MLSE (b)

Figure 5.1. DB codec with differential encoding (a) and with MLSE decoding (b) and spectral comparison

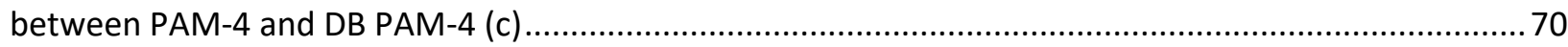
Figure 5.2. Experimental setup for testing DB FFE performance together with the spectrum of the DAC and system spectrums

Figure 5.3. Performance comparison between classic PAM-4 and DB PAM-4 at baud rates from 28 to 56

GBaud

Figure 5.4. Experimental setup for testing $100 \mathrm{Gbps}$ PAM transmissions.

Figure 5.5. 28 GBaud PAM-4 eye diagrams after DAC (a) and after ADC (b) and 19 GBaud PAM-8 eye diagrams after DAC (c) and after ADC (d)

Figure 5.6. 56 and 112 PAM-4 sensitivity vs. BER curves (a) and PAM-4 vs. PAM-8 sensitivity performance comparison (b). DSP: 81-tap FFE followed by a 16-state (for PAM-4) or 64-state (for PAM-8) MLSE .......75 Figure 5.7. Block diagram of a basic optical transmission system ........................................................77

Figure 5.8. The spectrums for a 112 Gbps PAM-4 employing DPE .....................................................78

Figure 5.9. Experimental setup for testing DPE techniques at high baud rates ......................................79

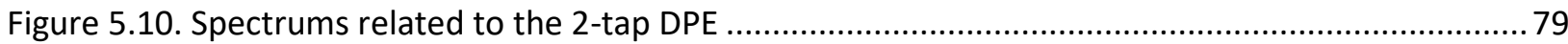

Figure 5.11. Sensitivity performance comparison between PAM-4, DB PAM-4 and DB PAM-4 with a 2-tap DPE filter (a) and FFE complexity vs. performance curves for DB PAM-4 with and without DPE (b). All results are for a $112 \mathrm{Gbps}$, BTB transmission

Figure 5.12. Spectrum comparison (for a 28 Gbps transmission) between OOK, DB and PRS (a) and CD tolerance comparison (for the same $28 \mathrm{Gbps}$ transmission) between OOK, DB and PRS in low (10 GHz bandwidth) and high bandwidth ( $20 \mathrm{GHz}$ bandwidth) scenarios (b)....

Figure 5.13. Pulse shapes (first column) for OOK (first row), DB (second row), and PRS (third row) and the corresponding eye diagrams for BTB (second column), $15 \mathrm{~km}$ (third column), and $30 \mathrm{~km}$ (fourth column).

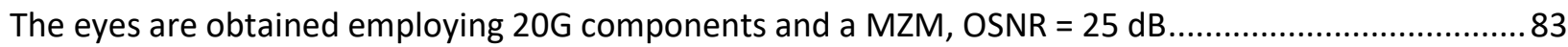

Figure 5.14. Symbol mapping for different modulation/modulator combinations................................. 84

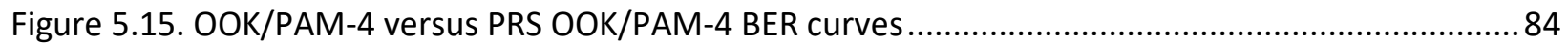

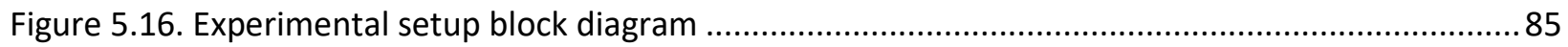

Figure 5.17. OOK versus PRS transmission length (a) and OSNR sensitivity curves for OOK and PRS for

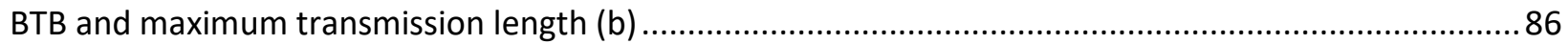

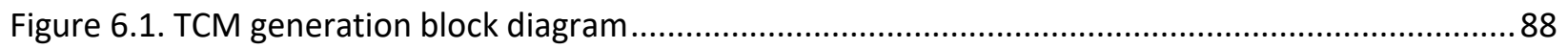

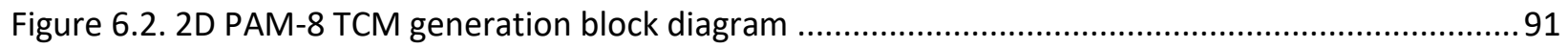

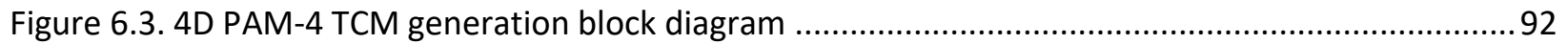

Figure 6.4. 3D PAM-4 TCM constellation representation by subsets .................................................93

Figure 6.5. Convolutional encoder block diagram ......................................................................... 94 
Figure 6.6. Simulation comparison between PAM-4 and 8-state and 16-state convolutional code 4D

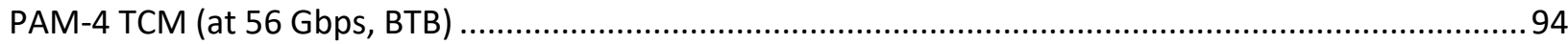

Figure 6.7. 4D PAM-4 TCM vs. PAM-4 experimental setup block diagram ...........................................95

Figure 6.8. Performance comparison between 8-state and 16- state TCM PAM-4 (before and after trellis decoder) and PAM-4. BER curves are obtained by processing the experimental data (a) and by tail extrapolation (b). BER curves for PAM-4 and 8-state TCM for BTB, 10, 20, 30 and $40 \mathrm{~km}$ (c) and for 21,

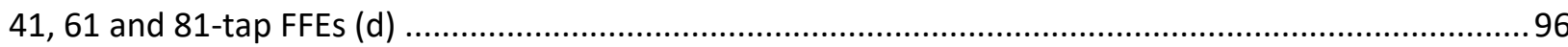

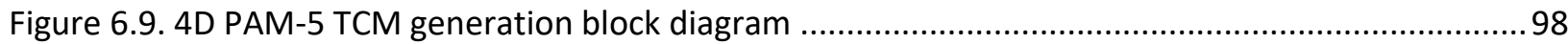

Figure 6.10. 3D PAM-5 TCM constellation representation by subsets ....................................................99

Figure 6.11. Experimental setup for testing the 4D PAM-5 TCM ....................................................99

Figure 6.12. The transfer function of a simulated PAM-4 signal, of the DAC, and of the overall system ..99 Figure 6.13. Eye diagrams of $4 D$ PAM- 4 and PAM-5 TCMs, after DAC, after ADC and after FFE. The eyes presented in this figure are obtained for a $56 \mathrm{Gbps}$, BTB transmission 100 Figure 6.14. The number of FFE taps versus the input power required to obtain a BER of $3 \times 10^{-4}$ for PAM4, PAM-4 TCM and PAM-5 TCM schemes (a) and Comparison of performance for TCM and PAM-4 with and without MLSE (b) 102

Figure 6.15. Comparison of performance of PAM-4 TCM (squares), PAM-5 TCM (triangles) and of PAM-4 (circles) for BTB (a) and links of $10 \mathrm{~km}$ (b), $20 \mathrm{~km} \mathrm{(c),30} \mathrm{km} \mathrm{(d)} \mathrm{and} 40 \mathrm{~km}$ (e) at $56 \mathrm{Gbps}$ 103 Figure 6.16. Power sensitivity comparison between 4D PAM-4 and PAM-5 TCM and PAM-4 at 56 (a), 64 (b), 72 (c), 80 (d), 88 (e), 96 (f), 104 (g), $112 \mathrm{Gbps}(\mathrm{h})$, and the gain of TCM over PAM-4 at bit rates between 56 and 112 Gbps (i) 104

Figure 7.1. Multidimensional set-partitioned modulation generation block diagram ........................... 107

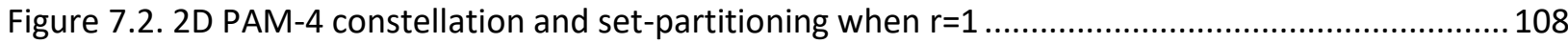

Figure 7.3. The 2D PAM-8 subsets (a) and 2D DB PAM-8 subsets after the receiver (b)....................... 109

Figure 7.4. 3D PAM-8 set-partitioning modulation generation block diagram ....................................... 110

Figure 7.5. Performance comparison between PAM-8 and 3D PAM-8 in the presence of noise and without bandwidth constraints

Figure 7.6. Performance comparison between PAM-8 and 3D PAM-8 (continuous lines) and DB PAM-8

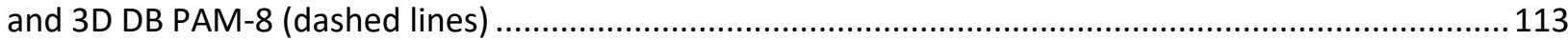

Figure 7.7. 3D PAM-8 experimental setup and system transfer function 114

Figure 7.8. BER performance comparison between PAM-8 and 3D PAM-8 for bit rates from 56 to 180 Gbps (continuous lines) and between DB PAM-8 and 3D DB PAM-8 for bit rates between 180 and 240 Gbps (dashed lines)..... 


\section{List of tables}

Table 1. Gray coding and mapping for OOK, PAM-4, PAM-8 and PAM-16 .........................................50

Table 2. Optimum modulation levels for minimizing the effects of optical noise...................................55

Table 3. The experimental normalized amplitude values for ESL, s-ESL and OSL PAM-4........................60

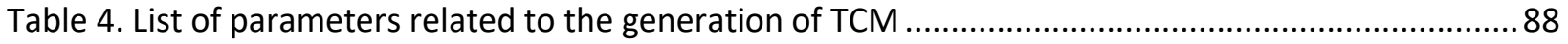

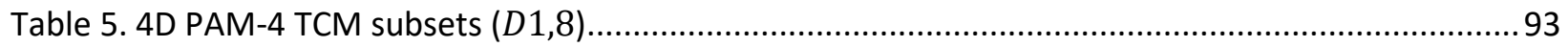

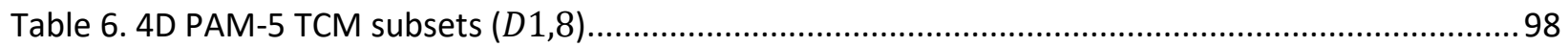

Table 7. The dmin and baud rate in relation with the number of redundancy bits for the 4D PAM-4 scheme 


\section{Nomenclature}

\begin{tabular}{|c|c|}
\hline $3 D$ & 3-dimensional \\
\hline 4D & 4-dimensional \\
\hline ADC & analog-to-digital converter \\
\hline AGC & automatic gain controller \\
\hline ASIC & application specific integrated circuit \\
\hline AWG & arbitrary waveform generator \\
\hline AWGN & additive white Gaussian noise \\
\hline $\mathrm{BCH}$ & Bose-Chauduri-Hocquenghem \\
\hline BER & bit-error rate \\
\hline BPG & bit-pattern generator \\
\hline BTB & back-to-back \\
\hline Bw & bandwidth (used only in figures to save space) \\
\hline CAP & carrier-less amplitude phase modulation \\
\hline CAPS & combined amplitude-phase shift \\
\hline$C D$ & chromatic dispersion \\
\hline CW & continuous wave \\
\hline DAC & digital-to-analog converter \\
\hline DB & duobinary \\
\hline DC & direct current \\
\hline DCF & dispersion compensating fiber \\
\hline DCM & dispersion compensation module \\
\hline DFB & distributed feedback laser \\
\hline DFE & decision feedback equalizer \\
\hline DML & directly modulated laser \\
\hline DMT & discrete multi-tone \\
\hline DMUX & demultiplexer \\
\hline DPE & digital pre-emphasis \\
\hline DSP & digital signal processing \\
\hline EAM & electro-absorption modulator \\
\hline EDFA & erbium doped fiber amplifier \\
\hline EML & electro-absorption modulated laser \\
\hline ER & extintion ratio \\
\hline ESL & equally spaced levels \\
\hline FEC & forward error correction \\
\hline FFE & feed-forward equalizer \\
\hline FIR & finite impulse response \\
\hline GRC & Huawei German research center \\
\hline GVD & group-velocity dispersion \\
\hline
\end{tabular}




\begin{tabular}{|c|c|}
\hline IIR & infinite impulse response \\
\hline IM-DD & Intensity modulation and direct detection \\
\hline ISI & inter-symbol interference \\
\hline LMS & least mean squared \\
\hline LPF & low-pass filter \\
\hline LSB & least significant bit \\
\hline LTID & linear time-invariant discrete \\
\hline $\max$ & maximum \\
\hline MD & modulation driver \\
\hline MLSE & maximum likelihood sequence estimation \\
\hline MSB & most significant bit \\
\hline MSE & mean-square error \\
\hline MUX & multiplexer \\
\hline MZM & Mach-zehnder modulator \\
\hline NF-OOK & narrowly filtered on-off keying \\
\hline NLSE & nonlinear Schrödinger equation \\
\hline NRZ & non-return to zero \\
\hline OBF & optical bandpass filter \\
\hline OFDM & orthogonal frequency division multiplexing \\
\hline OOK & on-off keying \\
\hline OSA & optical spectrum analyzer \\
\hline OSL & optimally spaced levels \\
\hline OSNR & optical signal-to-noise ratio \\
\hline PAM-4 & pulse amplitude modulation 4 \\
\hline PAPR & peak-to-average power ratio \\
\hline PB & polybinary \\
\hline PD & photodiode \\
\hline PMD & polarization-mode dispersion \\
\hline PRBS & pseudo-random bit sequence \\
\hline PRS & partial response signaling \\
\hline PtP & peak-to-peak \\
\hline ROSA & receiver optical sub-assembly \\
\hline RS & Reed-Solomon \\
\hline RTO & real-time oscilloscope \\
\hline s-ESL & squared equally spaced levels \\
\hline SNR & signal-to-noise ratio \\
\hline SOVA & soft output Viterbi algorithm \\
\hline SSMF & standard single-mode fiber \\
\hline TCM & trellis coded modulation \\
\hline TEC & temperature controller \\
\hline TIA & trans-impedance amplifier \\
\hline TOSA & transmitter optical sub-assembly \\
\hline UPV & universitat politecnica de Valencia \\
\hline
\end{tabular}


USL unequally spaced levels

VCSEL Vertical-cavity surface-emitting laser

VOA

variable optical attenuator

WDM

wave division multiplexing

$\lambda$

wavelength

$d_{\min }$

minimum distance 


\section{Chapter 1 Introduction}

\subsection{Motivation and state-of-the-art}

Since the early days of telecommunications there has been a demand for faster data transmissions and larger transmission capacity. This 'status quo' has not changed too much until now. Although modern telecommunication systems have the ability to transmit very large amounts of data, much larger than in the early days of telecom, it never seems to be enough to satisfy demand [1]. As such there is still room for research and improvement in this area. A major development in the field of telecommunications was the introduction of optical transmissions over standard single-mode optical fiber. Nowadays, optical transmission systems provide the backbone of telecom networks. They are employed anywhere from intercontinental transmissions and down to metro access and inter- and intra-datacenter networks. For long range transmissions, i.e. longer than $80 \mathrm{~km}$, optical communications systems employ coherent solutions (i.e. all the characteristics of the transmitted signal are recovered by the receiver - power, phase, polarization) due to their reduced signal-tonoise ratio (SNR) requirements. For short-reach optical networks intensity-modulation and direct detection (IM-DD) implementations are preferred. Coherent transmissions provide better quality and higher capacity; however, they are also more expensive and require higher complexity signal processing at the receiver side. While for long distance connections only the coherent solution can provide good enough performance, for short-reach transmissions, where the fiber dispersion and attenuation values are relatively low, non-coherent implementations can replace the more expensive coherent solutions. The short-reach optical infrastructure has been expanding in the last years, being pushed by metro-access networks and more significantly by intra- and inter-datacenter connections, which are supporting the cloud infrastructure [2], as can be observed in Figure 1.1. These networks require very high volumes of optical transceivers, making the price of such devices the number one priority. That is why in these scenarios the non-coherent approach is preferred, as, at least for the time being, it is significantly less expensive than its coherent counterpart. Since price is the number one concern in non-coherent optical systems, the best components are not always employed which can sometimes lead to unacceptable performance. To solve this problem DSP and FEC are needed. In order to guarantee bit-error rates (BER) below a certain threshold (usually the BER should be below $10^{-15}$ ) FEC is required. 


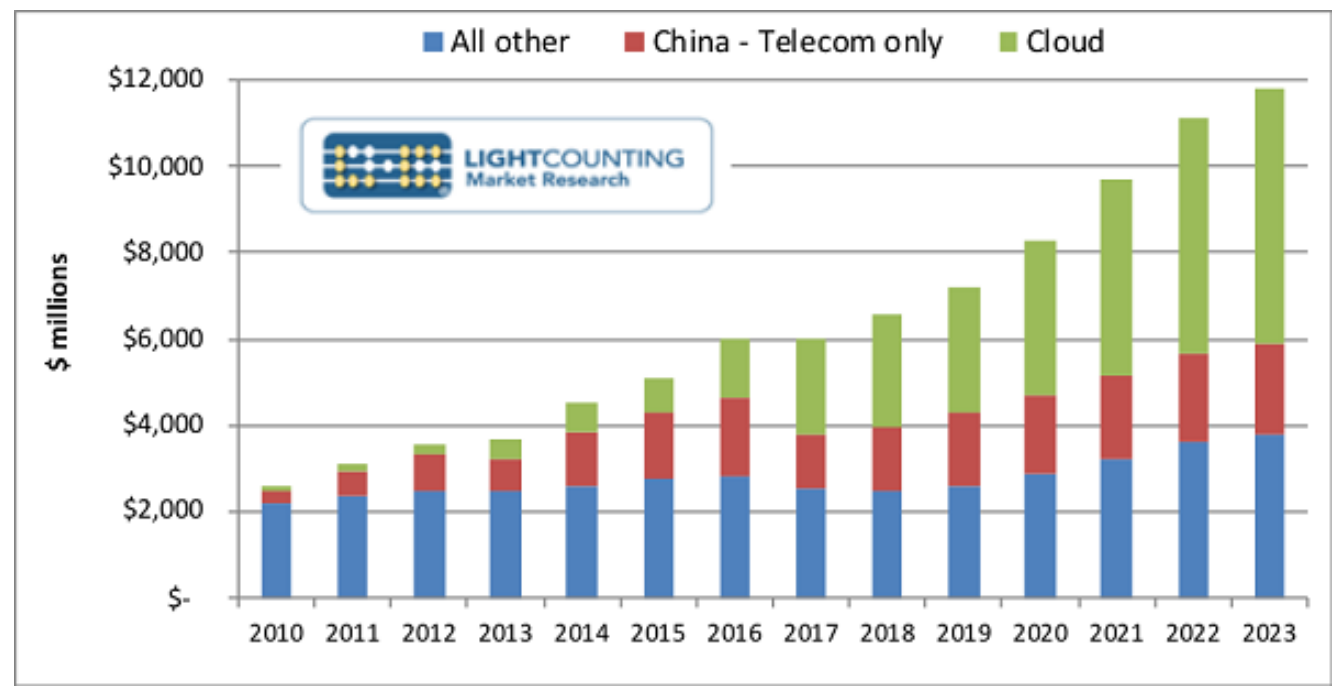

Figure 1.1. Optical components sales (history and prediction - 2010 to 2023) [3]

To achieve the optical transmission BER requirements, any FEC code requires also that the BER of the signal before error correction is below a certain threshold, referred here to as FEC threshold. This threshold depends on the capabilities of the FEC, more powerful codes with higher overhead can have FEC BER thresholds of more than $10^{-2}$ but will consume a lot of resources. Most often the thresholds of $4 \times 10^{-3}$ or $3 \times 10^{-4}$ are employed [4], [5]. These error correction requirements imply that the received optical signal should have a BER below the FEC thresholds. However, given the high transmission bitrates used today (25 Gbaud/lambda or more) and the limited bandwidth of the components, the quality of the received signal of an IM-DD transmission will almost never be good enough to satisfy the FEC requirements. Here is where the DSP comes in; DSP algorithms are employed to reduce the BER below the threshold required by the FEC while enabling us to employ low-cost components. As such DSP becomes an essential and integral part of any state-ofthe-art non-coherent optical system. In recent years a lot of attention has been given to this area in the optical communications community. Due to its importance in modern non-coherent optical systems and the high potential from a research point of view, the subject of DSP in optical IM-DD transmissions has been chosen as the main topic for this thesis.

Current state-of-the-art commercially available non-coherent optical transceivers operate at 100 or 400 Gbps. While the 100 Gbps version has been thoroughly researched and standardized, the $400 \mathrm{Gbps}$ architecture is still being revised and standardization efforts have only recently begun [6]. The basic structure of these transceivers can be observed in Figure 1.2. In order to reach these high transmission bitrates (i.e. 100 and $400 \mathrm{Gbps}$ ) multiple channels are multiplexed together. The standard multiplexing technology employed in optical communications is the WDM, where each channel is transmitted on a slightly different wavelength of the light [7]. In modern IM-DD optical systems four wavelengths are usually multiplexed, giving us a data rate per channel of 25 or 100 Gbps for the 100 or 400 Gbps transceivers, respectively. Obviously, the $25 \mathrm{Gbps} / \lambda$ is much easier to achieve than the $100 \mathrm{Gbps} / \lambda$, thus it makes use of different, less expensive components and less complex DSP. 


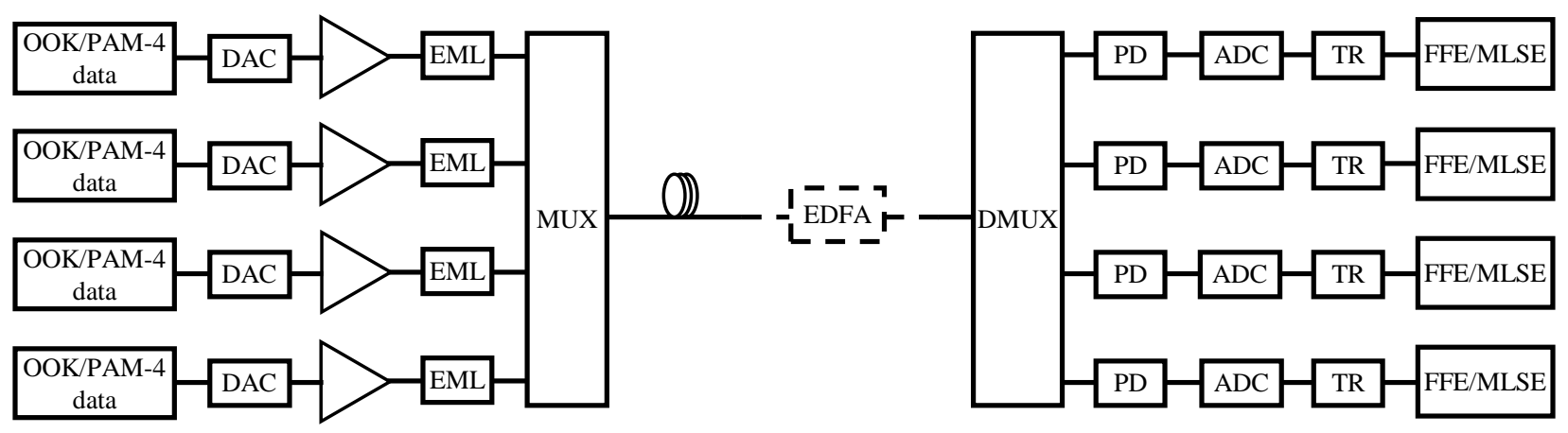

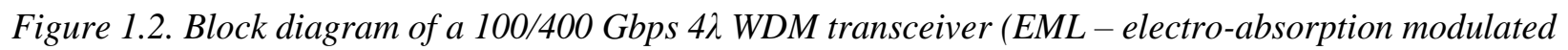
laser, MUX - multiplexer, DMUX - demultiplexer, EDFA - erbium doped fiber amplifier, TR -timing recovery)

For a non-coherent optical transmission, the main bandwidth limiting components are the digitalto-analog and analog-to-digital converters (DAC/ADC), the modulator and the photodiode (PD) receiver. The 100 Gbps systems typically employ the $10 \mathrm{G}$ generation (refers to components with a 3-dB bandwidth of around $10 \mathrm{G}$ or slightly higher) of components. To keep the system complexity as low as possible the basic on-off keying (OOK) modulation is used. For transmissions below 10 $\mathrm{km}$ the DSP consists of just a FFE, where 11 taps or less are usually sufficient to equalize the received signal. The $100 \mathrm{Gbps}$ transceivers are employed also over longer distances of $40 \mathrm{~km}$ [8]. In these scenarios the amount of CD generated by the link is very high. As we will see in chapter 3 , the FFE is very sensitive to dispersion and thus is incapable of bringing the BER below the FEC threshold. That is why for longer links the MLSE algorithm is preferred, as it can compensate better the effects of CD (at the cost of increased DSP complexity). The DSP structure of the transceiver gets more complex for the $400 \mathrm{Gbps}$ scenario. There are a couple of big issues that come up when tackling such high-speed optical transmission systems. First issue is that, while the data rate requirements are quadrupled, the bandwidth of the components should be only double (referred to as $20 \mathrm{G}$ components) that of the components used for the $100 \mathrm{Gbps}$ transceivers. The second major problem is that the amount of dispersion generated by the link grows by the squared baud rate. Thus, if we were to employ the same modulation as in the $100 \mathrm{Gbps}$ case, the required baud rate would be four times higher for the $400 \mathrm{Gbps}$ transceivers resulting in 16 times the amount of $\mathrm{CD}$, effectively reducing transmission distance by 16 times. The first way of tackling this problem is to investigate other, more complex, modulation formats. Most of the research into this topic has been targeted towards the pulse-amplitude modulation 4 (PAM-4) [9], [10], the discrete multi-tone (DMT) modulation [11], which can be regarded as the non-coherent version of the orthogonal frequency-division multiplexing (OFDM) technology, and the carrier-less amplitude phase modulation (CAP) [12]. Due to its relatively low complexity (when compared with the other two options, making standardization efforts easier) and good performance, PAM-4 is currently the preferred solution [6]. In the early stages of this thesis it was assumed that PAM-4 will be the chosen modulation for the 400 Gbps generation of transceivers and thus most of the research efforts have been focused around the PAM-4 or other modulations derived from PAM-4. By employing PAM-4 the required baud rate is halved when compared with the OOK modulation. 
Thus, in order to achieve a bit rate of $100 \mathrm{Gbps} / \lambda$, a 50 GBaud PAM-4 signal is sufficient. In such a way the required bandwidth is only two times higher than that required by the $100 \mathrm{Gbps}$ systems, instead of four times, and the amount of CD in the link is increased by only four times, whereas with OOK it would have been increased by 16 times. The increase in the number of amplitude levels from 2 with OOK to 4 with PAM-4 does not come only with benefits. The higher number of levels translates into lower spacing between levels and thus a lower tolerance to noise. Since in the move from 100 to $400 \mathrm{Gbps}$ the effects of bandwidth, dispersion and noise are all amplified, the DSP that has been employed for the $100 \mathrm{Gbps}$ systems is no longer sufficient. One approach is to increase the power of the FFE and MLSE by increasing the number of taps or states respectively and to combine these two DSP algorithms together [13]. The main part of this work has been dedicated to investigating other DSP methods of improving performance, methods such as digital pre-emphasis (DPE) [14], DB signaling [15] or TCM [4].

The advance to $400 \mathrm{Gbps}$ transceivers has been difficult. The question of where should be the crossing point from non-coherent to coherent has been raised again. There is no consensus yet on this issue, however it is the opinion of the author that the higher the bit rate the lower this crossing point will be. While the $100 \mathrm{Gbps} / \lambda$ systems are still being worked on, research focus is shifting towards the next generation of transceivers which is aiming for a $200-250 \mathrm{Gbps} / \lambda$ bit rate. While there is still room for IM-DD systems in this new $800 \mathrm{Gbps}$ to 1 Tbps generation, the maximum distance that it will cover will probably be less than $10 \mathrm{~km}$ and its main focus would be on interand intra-datacenter applications. Since the datacenter market is very large and still growing and it requires high volumes of transceivers, it is more than enough to justify further research and investment into non-coherent solutions.

\subsection{Thesis overview}

This Ph.D. Thesis has been developed jointly by the Huawei German Research Center (GRC, formerly named European Research Center - ERC) and by the Nanophotonics Technology Centre (NTC) of the Universitat Politècnica de València (UPV). The objectives of this dissertation have been aligned to match both academic and industrial criteria and are the fruit of a continued joint work. As should be apparent from the previous section the focus of the work presented here is on DSP algorithms for short-reach IM-DD optical transmission systems. The main two objectives were to research DSP which could be employed in non-coherent optical transceivers in order to increase transmission rates and reduce the cost of the equipment and to disseminate the research in top-tier conferences and journals in order to increase the visibility of the NTC's Optical Networks Group and of Huawei's GRC Optical and Quantum Technologies department (the teams which the author was a member of during this Ph.D. research).

The thesis is split into a total of eight chapters, each focusing on a specific topic. A more detailed description of each chapter is presented below: 
- Introduction: in the first chapter the goals of the thesis are discussed. It starts by introducing the motivations behind this work and then a brief picture of the state-of-the-art is presented. In the second part of this chapter an overview of the thesis is given.

- IM-DD optical transmission systems: before going into details about DSP algorithms an overview of the non-coherent optical transmission systems is presented. The essential components of these systems are introduced, being split into three areas - transmitter components, optical link and receiver components. Since there are plenty of books giving a wider overview of the components, in this chapter the focus is on the role of each component in an experimental setup and on how they can be used, providing information that was hard to find by the author and would have been very useful at the beginning of the $\mathrm{PhD}$.

- Receiver digital signal processing: in this chapter the most widely used (in the context of IM-DD optical systems) receiver DSP techniques are presented; namely the FFE and the MLSE. Their capabilities and performance are analyzed and some of the early simulation and experimental results (employing FFE and/or MLSE) are shown.

- DSP techniques for multilevel modulation formats: this thesis is focused mainly on multilevel intensity modulation formats (any intensity modulation with more than two amplitude levels, e.g. PAM-4, PAM-8). This chapter starts by introducing the generation process of multilevel modulations. Next the performance of MLSE in the context of multilevel modulation is analyzed. The second half of this chapter takes a look at DSP techniques which apply only to multilevel modulation formats, namely geometrical and probabilistic shaping. Constellation shaping is a hot topic in coherent optical transmissions and it is shown here that it can be adapted for IM-DD systems and that it provides significant gains in terms of OSNR sensitivity and CD tolerance, requiring very low implementation complexity.

- Partial response signaling: the most critical limitation of high-speed short-reach noncoherent optical systems is the limited bandwidth of components. In this chapter some partial response signaling techniques, like DB and DPE, are investigated to combat the effects of low-bandwidth components. For both of these approaches important sensitivity gains are demonstrated when employed in conjunction with FFE and/or MLSE. In the last section of this chapter it is shown that partial response signaling can be used in noncoherent optical transmissions to also mitigate the effects of $\mathrm{CD}$.

- Trellis coded modulation: while the focus of this thesis is not FEC, in this chapter it is shown that error correction elements can be integrated into the modulation process in order to improve overall performance, generating a multidimensional multilevel modulation format. This modulation is referred to as TCM. In the first part of this chapter the generalized generation of TCM is presented while in latter part two particular examples of TCM are introduced: the 4-dimensional (4D) PAM-4 TCM and the 4D PAM-5 TCM. Their 
performance is analyzed in both simulations and experimentally, demonstrating the advantages and drawbacks of TCM.

- Multidimensional multilevel set-partitioned modulation: in this chapter a modified version of TCM is introduced. This so-called multidimensional set-partitioning modulation does not employ any FEC elements like TCM does, greatly reducing the complexity of this technique. While the maximum achievable sensitivity gains are lower than those of TCM, the modulation introduced in this chapter can be used in a wider range of transmission scenarios. After presenting the basics of the multidimensional multilevel set-partitioned modulation a specific implementation of said modulation is examined, namely the 3dimensional (3D) PAM-8 set-partitioning modulation. The performance of this modulation is analyzed both theoretically and experimentally, and the results of this analysis are shown in the last section of this chapter.

- Conclusions: the most valuable conclusions drawn during this $\mathrm{Ph} . \mathrm{D}$. research are summarized here and some paths for further improvement of results are suggested. 


\section{Chapter 2}

\section{IM-DD Optical Transmission Systems}

In this chapter the main components required for an IM-DD optical transmission are introduced. These can be split into three groups: transmitter side, optical channel and receiver side components. The block diagram of a basic optical IM-DD transmission is presented in the figure below. The continuous black line blocks represent components that are always required, while the dashed blue line is used for components which are relevant only for specific scenarios (they will be discussed later in this chapter).

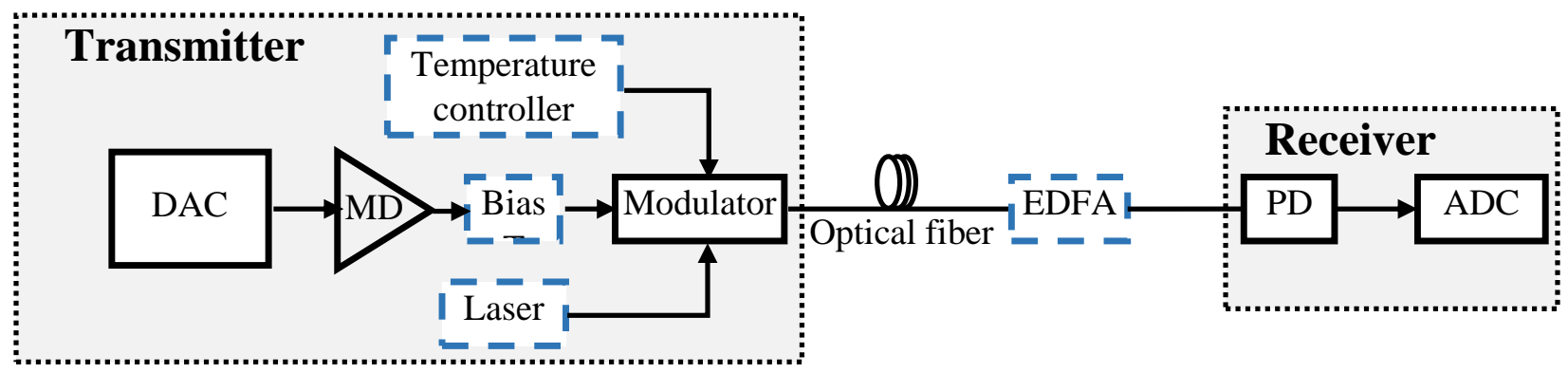

Figure 2.1. Block diagram of a basic optical transmission system (MD-modulator driver, EDFAerbium doped fiber amplifier)

Each block is approached initially from a theoretical point of view. Afterwards, a MATLAB (MATLAB combines a desktop environment tuned for iterative analysis and design processes with a programming language that expresses matrix and array mathematics directly [16]) model is constructed, where its functionality and limitations are simulated. In this way different DSP algorithms can be tested in simulation in order to prove their viability, before any experiments are carried out. It is worth mentioning that sometimes the mathematical model of various components is not $100 \%$ accurate or it does not consider all the properties of said components. That is why promising simulation results might not always translate well in practice and thus it is always necessary to experimentally verify these results. For each of the blocks in Figure 2.1 the various components employed in experiments are presented and their advantages and disadvantages are discussed in this chapter.

\subsection{Transmitter components}

Contemporary optical systems operate at very high bit rates, usually exceeding 28 Gbps per lambda. At these high speeds all electrical components introduce bandwidth limitations to some 
degree. That is why it is more convenient and more cost effective to implement the signal processing in the digital domain. In Figure 2.2 the essential transmitter side DSP blocks are displayed. Note that the transmitter DSP is not limited to these blocks and additional algorithms can be added (e.g. digital pre-emphasis) in order to improve performance.

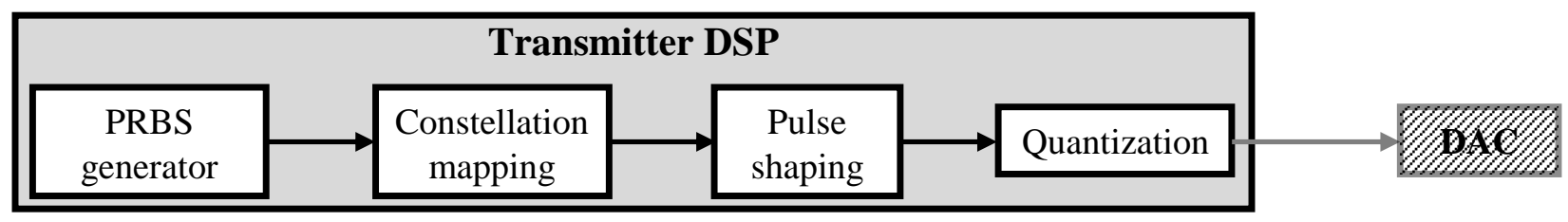

Figure 2.2 . DSP blocks of a basic communication transmitter

When simulating an IM transmitter, the first step is to generate a pseudo-random bit sequence (PRBS). A basic MATLAB function (e.g. rand) can be employed to generate the bit sequence. The length of the PRBS should be around $\left(2^{15}-1\right) *\left(N_{\text {bits per symbol }}\right)$ bits or longer in order to approximate a real transmission where the bit sequence is not cyclical. Otherwise the performance of a simulation or experiment might appear better than the real implementation, which is undesirable [17]. The next step is to map the PRBS onto the modulation constellation. If the OOK modulation is used then this step is not necessary. After the modulation symbols are obtained, pulse shaping is performed. The last step before the data is converted into the analog domain is to quantize the signal. The number and values of the quantization levels is dependent on the DAC.

\subsubsection{Digital-to-analog converter}

The DAC is an essential component of any digital communication system. When simulating the DAC there are several impairments that have to be considered. The most critical problem with DACs in the case of high-speed optical communications is the limited bandwidth. Most commercially available DACs have a 3-dB bandwidth somewhere between 10 and $20 \mathrm{GHz}$, while current transmission rates exceed 28 GBaud. Another issue is the limited number of bits used to quantize one sample (i.e. quantization noise). The lower the number of bits quantizing one sample (i.e. the resolution of the DAC) the higher the quantization noise. There is an inverse proportional relation between the bandwidth of the DAC and its resolution. Another important parameter of the DAC is the sampling rate (i.e. number of samples per symbol). The higher it is the more accurately the digital signal can be reproduced by the DAC.

During this thesis several types of equipment were employed to fulfill the role of DAC. One such equipment is the bit-pattern generator (BPG). Unlike regular DACs, the BPG does not require any of the DSP shown in Figure 2.2. Its output is a pre-determined stream of bits (it is basically a PRBS generator) meaning it can generate only the OOK modulation. This would be the equivalent of a DAC with a resolution of just one bit. The SHF 12103A BPG [18] used in our experiments has a bandwidth of more than $25 \mathrm{GHz}$ (the vendor does not provide an exact number or a transfer function curve, this number is estimated from our experimental results). It has four differential outputs that can generate a bit stream at rates between 3 and $28 \mathrm{Gbps}$ and two more multiplexed 
differential outputs that go up to $56 \mathrm{Gbps}$. In order to operate the BPG an external clock source is required. A clock signal of $28 \mathrm{GHz}$ is needed to run the BPG at the maximum data rate (i.e. 56 Gbps). In the figure below some sample eye diagrams at the output of the BPG are shown:
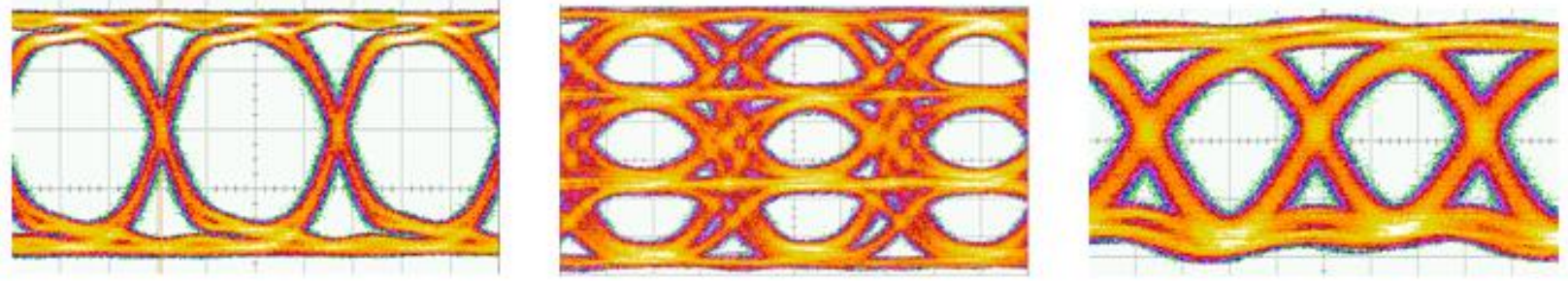

Figure 2.3. Eye diagrams at the output of the BPG (SHF 12301A): 28 GBaud OOK (left), 28 GBaud PAM-4 (middle) and 56 Gbaud OOK (right)

The main drawback of the BPG is that signal pre-processing (e.g. digital pre-emphasis) is not possible at the transmitter side. All of the basic functions that normally would be implemented in MATLAB, like PRBS generation or pulse shaping are done by the equipment, and more advanced techniques are not possible. Another issue is that the output of the BPG is an OOK non-return to zero (NRZ) signal. In order to generate a higher order modulation (e.g. PAM-4) some extra components are required, as illustrated in Figure 2.4. Depending on baud rate they will add extra bandwidth limitations in the systems. Furthermore, if the amplitude of the signal at the output of the BPG is too large then the power combiner will add some undesired reflections. An example of a PAM-4 signal generated with this method is shown in Figure 2.3 (the middle eye diagram). The highest bit rate can be achieved by adding the two 56 Gbps outputs in order to generate a 112 Gbps PAM-4 signal.

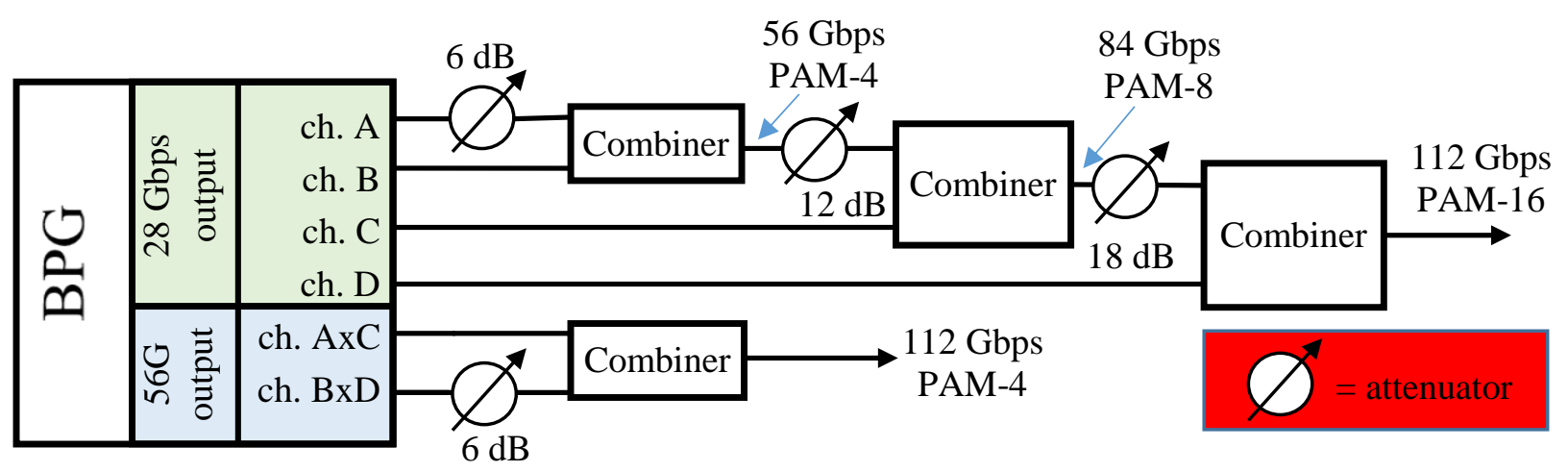

Figure 2.4. Generation of higher order modulations with the BPG

Because of the drawbacks presented previously, the BPG is of limited use from a transmitter DSP point of view. That is why most of the experiments carried out for this thesis were done using a Fujitsu DAC with 8-bit resolution and a sampling rate of $84 \mathrm{GSamples/sec.} \mathrm{It} \mathrm{has} \mathrm{a} \mathrm{total} \mathrm{of} \mathrm{four}$ differential outputs (8 total output ports) and each of these channels can output a custom signal. The DAC requires an external clock source to operate. The clock should have a rate equal to the sampling rate of the DAC divided by 32 (i.e. $2.625 \mathrm{GHz}$ ). Because of the external clock source, 
some clock tones can be observed in the spectrum at the output of the DAC (Figure 2.5 - spike centered at $21 \mathrm{GHz}$ ). The memory is structured in blocks of 128 bytes and has a maximum capacity of $1 \mathrm{MB}$. This block structure means that the number of samples that are loaded must be a multiple of 128. The data loaded into the memory is repeated in order to create a continuous signal. The peak-to-peak (PtP) amplitude of the output signal is roughly $400 \mathrm{mV}$. The main limitation of the DAC is its bandwidth which stands at $10-15 \mathrm{GHz}$ (varies slightly depending on selected output channel - see Figure 2.5, right side). The transfer function of the converter and the eye diagrams at the outputs of the DAC are shown in Figure 2.5. It can be observed here that the quality of the eye varies from channel to channel. In our experiments the negative output from channel 2 was used, unless specified otherwise.
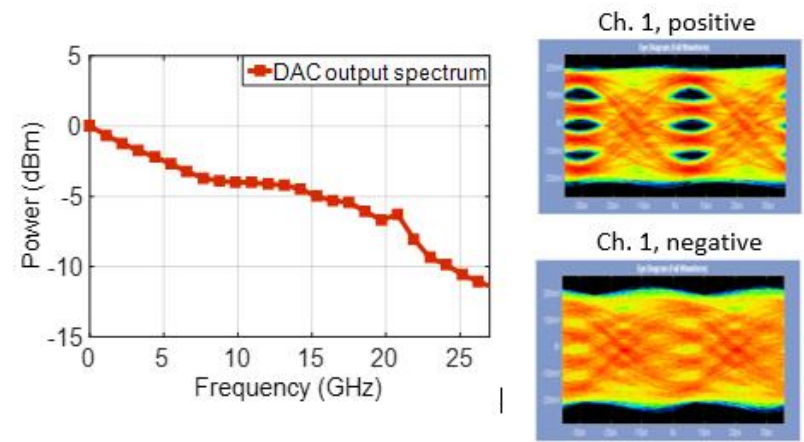

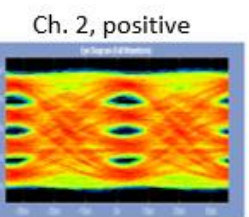

Ch. 2, negative

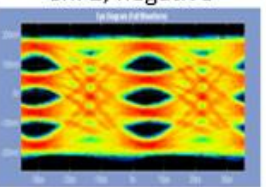

Ch. 3, positive

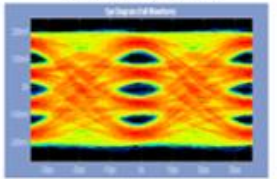

Ch. 3 , negative

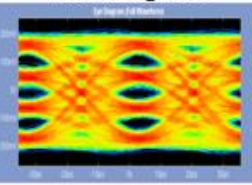

Ch. 4, positive

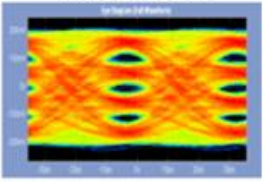

Ch. 4, negative

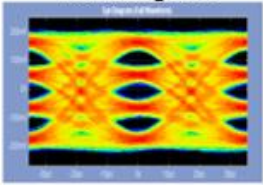

Figure 2.5. Fujitsu DAC transfer function (left) and the eye diagrams at the output of the Fujitsu DAC for each channel

With the 8-bit resolution a maximum of 256 amplitude levels can be represented. Normally higher order intensity modulation (i.e. PAM-16 or more) are impractical due to high SNR and optical signal-to-noise ratio (OSNR) sensitivity, thus at most three bits are necessary to generate the modulation levels. The rest can be employed by the transmitter DSP algorithms. The samples loaded into the DAC are quantized over 256 equidistant integer values, ranging from 0 to 255 . If a PRBS sequence $B$ (string of $O$ 's and 1 's) of length $m=N_{s} * m o$ (it must be a multiple of the modulation order, $m o$ ) is generated then the following process can be employed to generate the appropriate DAC data (see Figure 2.2 for a basic block view of this process):

$$
\begin{gathered}
m o=\log _{2}\left(N_{\text {levels }}\right), \quad N_{\text {levels }} \text { is the number of modulation levels } \\
S=\left[\begin{array}{cccc}
B_{1} & B_{2} & \cdots & B_{m o} \\
B_{m o+1} & B_{m o+2} & \cdots & B_{2 * m o} \\
\vdots & \vdots & \ddots & \vdots \\
B_{\left(N_{S}-1\right) * m o+1} & B_{\left(N_{s}-1\right) * m o+2} & \cdots & B_{N_{s} * m o}
\end{array}\right] \cdot\left[\begin{array}{c}
2^{m o-1} \\
2^{m o-2} \\
\vdots \\
2^{0}
\end{array}\right], \\
\text { where length }(S)=N_{S}=\frac{m}{m o} \\
N_{S p s_{D A C}}=\frac{D A C_{s r}}{B_{\text {rate }}}
\end{gathered}
$$




$$
\begin{gathered}
N_{S a m_{D A C}}=N_{S} \cdot N_{D A C_{s p s}} \\
N_{\text {Sam }_{D A C}}=N_{\text {Sam }_{D A C}}-\left[N_{S a m_{D A C}} \%(128 * 84)\right] \\
N_{S}=\frac{N_{S a m_{D A C}}}{N_{S p s_{D A C}}} \\
S=S_{1 \text { to } N_{S}},
\end{gathered}
$$

where $N_{s p s_{D A C}}$ is the number of samples per symbol of the DAC, $D A C_{s r}$ is the DAC sampling rate, $B_{\text {rate }}$ is the baud rate, $N_{S a m_{D A C}}$ is the number of values that are loaded in the DAC. Equation (6) gives us the final number of symbols to be loaded. The symbols vector from (2) needs to be cut down to the new $N_{S}$ value (equation (7)). This approach works only for integer baud rates (refers to the value in $\mathrm{GHz}$, not in $\mathrm{Hz}$ ). While the algorithm could be adjusted to account also for non-integer values, for most of these the required $N_{S a m_{D A C}}$ would be larger than the total memory of the DAC. Each symbol in sequence $S$ is repeated an integer number of times $N_{S p s_{T x}}$ (integer number $>=2$ ). At this point the pulse shaping can be applied, usually in the form of a Bessel filter or a raised cosine filter [19]. The most appropriate shaping depends a lot on the experimental setup (amount of nonlinearities in the system, number of samples per symbol at the output of the DAC, transmission length) and thus should be optimized for each experiment. After this the data is resampled to $N_{s p s_{D A C}}$ samples per symbol. If the whole process is done correctly the length of the data after the resampling should be equal to $N_{S a m_{D A C}}$. The last step before loading the data into the DAC is the quantization. If we denote the data before quantization as $T_{S a m}$ (transmitted samples) then:

$$
\begin{gathered}
A_{\text {max }}=\max \left(T_{\text {Sam }}\right) \\
A_{\text {min }}=\min \left(T_{\text {Sam }}\right) \\
T q_{\text {Sam }}=\text { round }\left[\left(\frac{T_{\text {Sam }}-A_{\text {min }}}{A_{\text {max }}-A_{\text {min }}}\right) *\left(2^{N_{\text {res }}}-1\right)\right]
\end{gathered}
$$

where $\max ($.$) is the maximum value of a vector, \min ($.$) is the minimum value of a vector,$ round(.) is the rounded value of a number or vector (i.e. $x . y=x$ if $y<0.5$ or $x+1$ if $y \geq$ $0.5)$ and $T q_{\text {Sam }}$ are the quantized values of $T_{\text {Sam }}$. The data loaded into the DAC are $T q_{\text {Sam }}$.

Due to the high resolution the effects of quantization noise are small. Furthermore the quality of the DAC output is not affected too much by the limited sampling rate (i.e. $D A C_{s r}$ ), although it does limit the maximum baud rate (at least one sample/symbol is required, thus the baud rate shouldn't be higher than $D A C_{s r}$, transmissions with less than one sample/symbol are possible but come with a high performance penalty). The biggest impediment when employing a DAC is its limited bandwidth. In simulations, in order to simplify implementation, only the bandwidth of the DAC is represented, while the quantization noise and other impediments are ignored. The limited 
bandwidth is represented by a low-pass filter (LPF), like the Bessel filter. Another more accurate method is to capture the transfer function of the DAC and use it instead of the LPF. While more accurate this approach is also more time consuming and is employed only in some specific scenario (i.e. when using bandwidth pre-compensation).

The last piece of equipment employed fulfilling the role of DAC is the arbitrary waveform generator (AWG), more precisely the Keysight M8196A AWG [20]. The AWG has a bandwidth of $32 \mathrm{GHz}$ and a sampling rate of up to $92 \mathrm{GSamples} / \mathrm{sec}$. At its core is a DAC very similar to the DAC presented in the previous paragraphs. However, this time the DAC is integrated with additional components which help increase the bandwidth of the equipment (see Figure 2.6, left side). Additionally, a MATLAB user interface is provided which allows a more streamlined user experience. Several modulation formats can be chosen through this interface. If a modulation that is not provided in the user interface is desired, then the user has access to the MATLAB code of the AWG and can implement it there. When doing this the same rules apply as in the case of the Fujitsu DAC (see equations (1) to (10)). The AWG allows us to compensate for the transfer function of the AWG itself and, if a Keysight real-time oscilloscope (RTO) is available, to compensate for the whole transmission system. Another advantage is that we can control the PtP voltage at the output, voltage which ranges from 80 up to $1000 \mathrm{mV}$. Due to the high bandwidth and slightly higher sampling rate the AWG is very well suited for very high-speed experiments (i.e. baud rate > 56 Gbaud). The improved performance of the AWG over the DAC comes however with some drawbacks - the cost, size and power consumption are all higher/bigger than those of the stand-alone DAC. As a result, the AWG is only viable for lab experiments and research and is not suitable for use in commercial products.
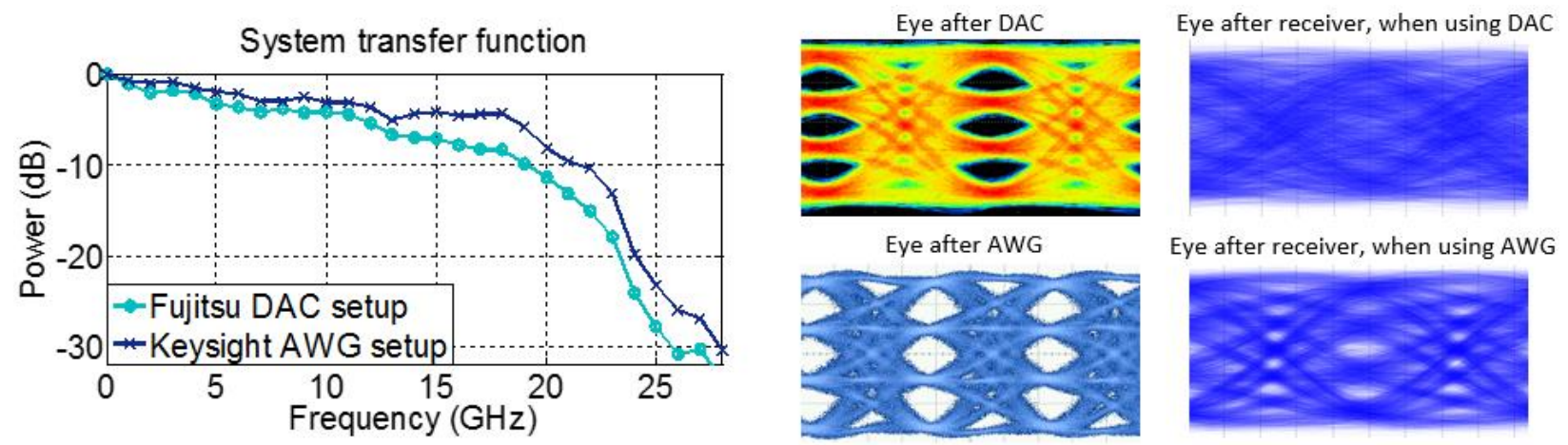

Figure 2.6. Spectrum comparison between two experimental setups, one using the DAC and the other the $A W G$ (the rest of the components are identical) (left side) and the back-to-back (BTB), 28 GBaud PAM-4 eye diagrams after DAC/AWG and after receiver for the same setups (right side)

\subsubsection{Modulator driver}

All the modulators which were used over the duration of this thesis require inputs with an amplitude of $2 \mathrm{~V}$ or more. If the signal has lower PtP amplitude then the full modulation range of the modulator is not used, which leads to higher SNR/OSNR sensitivity and in general worse performance. The equipment employed to generate the electrical signal cannot generate high 
enough PtP voltages (BPG - max. $750 \mathrm{mV}$, DAC $400 \mathrm{mV}$, AWG - max. $1000 \mathrm{mV}$ ), thus an extra amplifier is required between the DAC and the modulator in order to obtain an optimum optical signal. This amplifier is referred to in this work as modulator driver (MD). This component has usually a high bandwidth (50 GHz or even more for commercially available options) and good linearity. Typically, the signal is amplified by up to $24 \mathrm{~dB}$ by the MD. The driver also increases the noise level and causes a drop in SNR. For state-of-the-art amplifiers this drop is not very large, standing at about $0.3 \mathrm{~dB}$ for single stage amplifiers (e.g. SHF 827, they have lower gain, around 10-12 dB) and at $0.5 \mathrm{~dB}$ for two-stage amplifiers (e.g. SHF 807). Because some modulators or other equipment like oscilloscopes can be damaged if the input power is too large, it is very important to know the amplitude of the signal at the output of the MD. Knowing the amplitude of the signal at the input of the amplifier, the following formula can be used to calculate the amplitude at the output:

$$
A_{\text {out }}=A_{\text {in }} \cdot 10^{\frac{L}{20}}
$$

where $A_{\text {out }}$ is the amplitude of the signal at the output of the MD, $A_{\text {in }}$ is the amplitude of the signal at the input of the MD and $L$ is the amplification value in $\mathrm{dB}$. If the value of $A_{\text {out }}$ is too large some attenuators should be added to reduce the effective value of $L$. For some MDs the amplification value can also be varied.

\subsubsection{Modulator and auxiliary components}

The last step at the transmitter side after the signal has been amplified is to convert it into the optical domain. The component that has as input the electrical signal and as output an optical signal is generally referred to as an optical modulator. State-of-the-art research reports several devices capable of performing the optical modulation. These are: the Mach-Zehnder modulator (MZM) [21], [22], the electro-absorption modulated laser (EML) [14], [23], [15], the directly modulated laser (DML) [24], [25] and the vertical-cavity surface-emitting laser (VCSEL) [26], [27], [28]. The first two options are employed the most in contemporary short-reach commercially available transceivers, while VCSELs are favored for intra-datacenter networks. In this thesis the MZM and EML are the main modulators used in the experimental setups.

\subsubsection{Electro-absorption modulated laser}

The EML is composed out of two main components, a continuous wave (CW) laser source and an electro-absorption modulator (EAM), which are integrated together and usually put inside a hermetically sealed case. In order to obtain the best performance with such a device a constant temperature should be maintained inside its casing. That is why it is common practice to also integrate a thermistor and a cooler together with the laser and the modulator. The block diagram of an EML can be seen in the figure below [29]: 


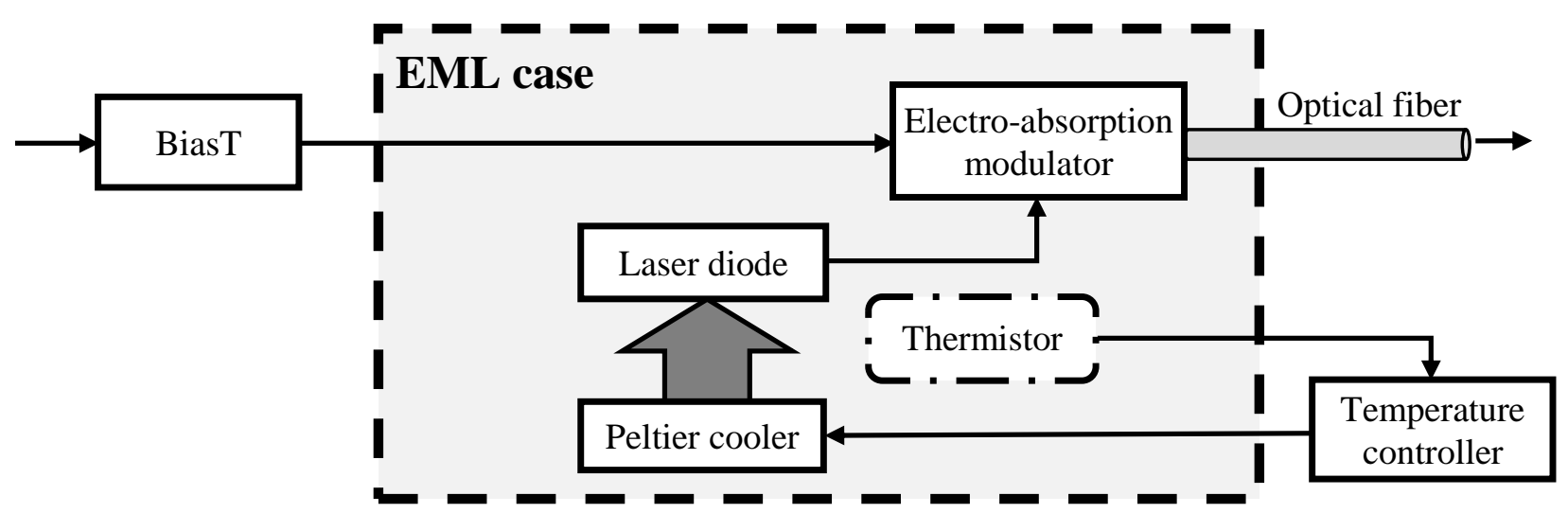

Figure 2.7. EML block diagram

In order to function properly the EML requires the use of two additional external components, a biasT and a temperature controller. The biasT is a circuit that sets the direct current (DC) component of a signal (see Figure 2.8(a)). The output signal from the DAC has a DC equal to 0. In order to position the signal properly on the transfer function (i.e. output power versus applied voltage curve) of the modulator a DC bias is often, if not always, required. Different modulation formats might need different biases also. Furthermore, the EAM can be seen as a reversed bias pi-n diode [30], meaning that a negative DC bias is necessary for it to function. Improper biasing of the signal at the input of the EML can lead to the damaging of the EAM. The performance of the EML is also dependent on the temperature. In order to adjust this an external device called temperature controller (TEC) is required. The TEC receives feedback from the thermistor which is integrated in the EML. The user has to set the desired temperature of the EML in the TEC. If the TEC setting and the thermistor feedback temperature are not the same the TEC sends back a signal to the EML's Peltier cooler, which in turn adjusts the temperature according to the feedback from the TEC. In order to save cost and power consumption, some EMLs can operate safely without the it.

The many elements of an EML and the multitude of interactions and physical effects which occur inside this type of device make it a very complex task to build an accurate simulation. However, a few approximations can be made and some effects can be ignored in order to greatly simplify the task at hand, without losing too much precision. The effects of temperature on the EML are not considered (the thermistor, cooler and TEC are left out of the simulation). Only the two main components are considered, the laser diode and the EAM. For the laser a simplified model is used which ignores such effects as the wavelength drift with temperature or side mode generation [31]:

$$
E_{\text {laser }}(t)=\left[E_{C W}(t)+R I N(t)\right] * \exp [1 j * \operatorname{laserPN}(t)]
$$

where $E_{\text {laser }}$ is the output optical field, $E_{C W}$ is a continuous wave signal ( $E_{C W}=$ $\left.\sqrt{10^{-3} * 10^{P_{\text {laser }} / 10}}[\mathrm{dBm}]\right), R I N$ is the relative intensity noise and laserPN is the laser phase noise. In most cases the model can be simplified even further without a significant accuracy loss, to the point where the output signal is a perfect continuous wave: 


$$
E_{\text {laser }}(t)=\sqrt{10^{-3} * 10^{P_{\text {laser }}(t) / 10}}[\mathrm{dBm}]
$$

The EAM operates by exploiting the lengthening of the wavelength of the absorption edge of a semiconductor in the presence of an electrical field [29], [32]. This effect is widely known as the Franz-Keldysh effect [33], [34]. In the ideal case the electric field would modulate only the absorption characteristic, however in reality it modulates also the refractive index [29] resulting in the generation of quadratic phase changes which are translated to a linear chirp in the simplest case, i.e. a linear instantaneous frequency change dependent on the wavelength. While in principle an undesired effect, negative values of the chirp can extend the transmission distance in the presence of CD (although the BTB performance would be worse). The chirp values of an EML are however much lower than in the case of a DML, this being a major reason why EMLs are generally preferred over DMLs.

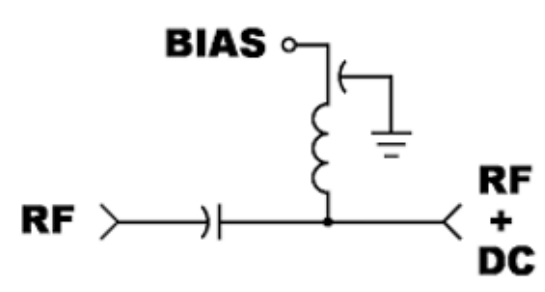

a)

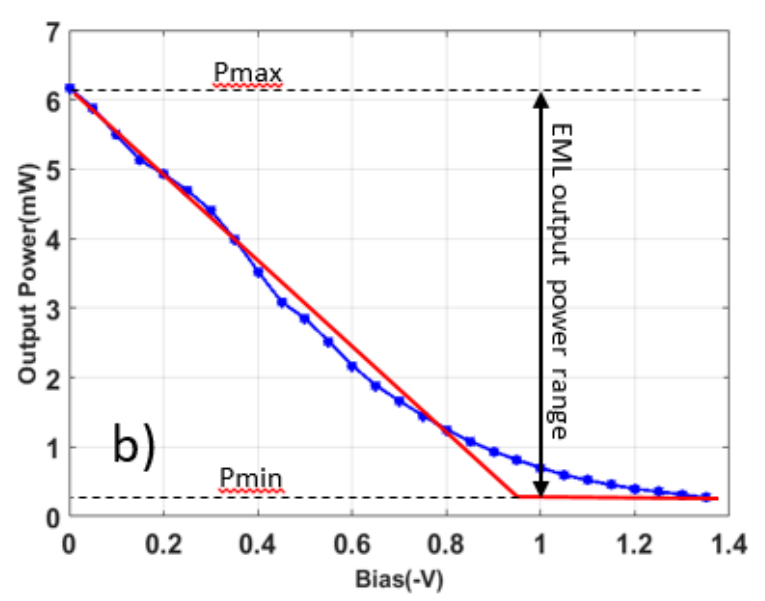

Figure 2.8. biasT circuit (a) and the bias versus output power curve of a commercially available $10 G$ Sumitomo EML (b)

In Figure 2.8(b) the relationship between the input electrical field and output optical power of an EML is shown. The curve can be considered linear for a bias between 0 and $-0.95 \mathrm{~V}$ (see the red line in Figure 2.8(b)). If the bias is decreased further the output power becomes saturated. An important parameter of all optical modulators related to this saturation power is the so-called extinction ration (ER), which is defined as the ratio between the maximum achievable power and the minimum (saturation) power:

$$
E R[d B]=10 * \log _{10}\left(\frac{P_{\max }}{P_{\min }}\right)
$$

The linear approximation of the modulator transfer curve implies that the relationship between the input electrical field and the output optical power is also linear. This means that, in its simplest form, the optical field at the output of the EML will be the square root of the input electrical field:

$$
E_{\text {opt }}(t)=E_{\text {laser }}(t) * \sqrt{E_{\text {el }}(t)}
$$


In experiments the ER of the modulator plays an important role in the overall performance, and thus it should be taken into consideration also for simulations.

$$
\begin{gathered}
e r=10^{\frac{E R[d B]}{10}} \\
E^{\prime}{ }_{e l}(t)=\frac{1}{e r}+\left(1-\frac{1}{e r}\right) *\left[\frac{E_{e l}(t)-\min \left(E_{e l}\right)}{\max \left(E_{e l}\right)-\min \left(E_{e l}\right)}\right] \\
E_{\text {opt }}(t)=E_{\text {laser }}(t) * \sqrt{E^{\prime}{ }_{e l}(t)}
\end{gathered}
$$

where $e r$ is the absolute value of the extinction ration and $E_{e l}$ is the electrical signal at the input of the EML. Another factor which should be taken into consideration, especially for simulations in the C-band, is the chirp. The complete equation for the EML, which takes into account both ER and chirp is given below [31]:

$$
E_{\text {opt }}(t)=E_{\text {laser }}(t) * \sqrt{E^{\prime}{ }_{\text {el }}(t)} * \exp \left[j * \frac{\alpha}{2} * \ln \left(E^{\prime}{ }_{\text {el }}(t)\right)\right]
$$

where $\alpha$ is the chirp factor coupling the amplitude and phase changes of the optical field [29] and $\ln ($.$) is the natural logarithm operator.$

When simulating an EML it is important to consider one more factor besides the laser and the EAM, that factor being the bandwidth of the device. The electric components of the EML limit its bandwidth. Most of the EMLs that were for this work have a 3-dB bandwidth ranging from 10 $\mathrm{GHz}$ up to $30 \mathrm{GHz}$. For high speed transmissions (i.e. 56 GBaud or more) even the latest models of EMLs introduce large bandwidth penalties. In simulation an additional LPF filter is needed after the implementation of equation (19) in order to reproduce the effects of the modulator bandwidth. A Bessel filter is chosen for the job, just as the one used when simulating the bandwidth limitations of the DAC.

EMLs are the most common solution for optical modulators employed in short-reach IM-DD systems. Their popularity is due to several factors, the most important ones being its small form factor and the small price of such a device. Compared to DML they have a much smaller chirp value and thus can operate at higher data rates. EMLs operating in both O-band and C-band are commercially available, with state-of-the-art devices having bandwidths of $25 \mathrm{GHz}$ or more.

\subsubsection{Mach-Zehnder modulator}

The most commonly used modulator in optical telecommunications is the MZM. The MZM is an interferometric device used to control the amplitude of an optical wave. It is mostly employed in coherent transmissions, although, due to its good performance and flexibility it is not uncommon to see it used also in IM-DD transmissions. Other advantages of the MZM over pure intensity modulators like the EML are that it enables the use of dispersion compensation techniques at the transmitter side and it allows for some more complex modulation formats (these aspects are 
discussed in more detail in later chapters). Furthermore, the MZM is less sensitive to temperature changes than the EML and thus does not require a TEC. The biasing voltage(s) can be applied directly to the component's pins such that an external biasT circuit is not needed. The biggest advantage of this type of devices is the modulation bandwidth, which can be in excess of $60 \mathrm{GHz}$, although most modern commercially available MZMs have bandwidths around 30 to $40 \mathrm{GHz}$. The MZM is not integrated together with a laser diode and thus an external laser source is required. The main drawback of MZM when compared to the other modulator alternatives for non-coherent optical communications is its higher price. In the figure below a basic block diagram of a transmitter employing a MZM is presented:

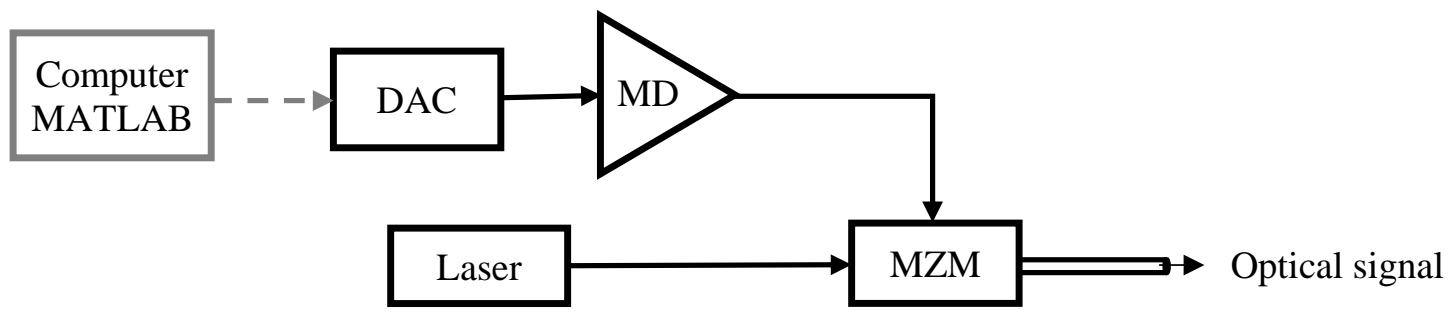

Figure 2.9. Basic block diagram of the transmitter side when employing a MZM

A MZM is an optical waveguide which splits into two arms. To each of the two branches a drive voltage is applied. After that the arms join back together to generate a single optical output (see Figure 2.10(a)). The variation of the applied drive voltage causes a change of the refractive index in the arms of the MZM which in turn will cause different phase shifts [35]. If no driving voltages are applied then the phase shift of the modulator signal should be the same for both arms, resulting in the same output as the input (i.e. constructive interference). When the phase shift difference between the two branches is equal to $\pi$ then no light is transmitted (i.e. destructive interference). The two voltages can be varied such as to obtain any output power level between 0 and the power of the input optical signal.

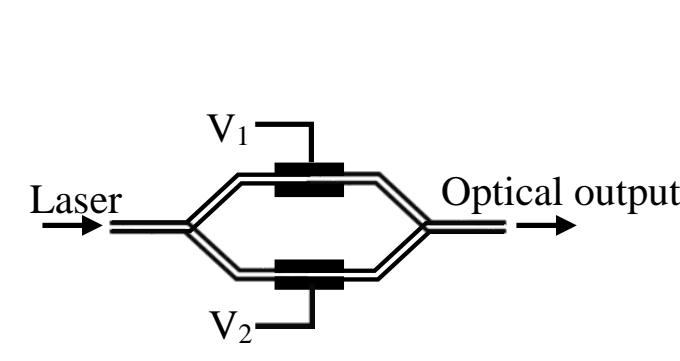

a)

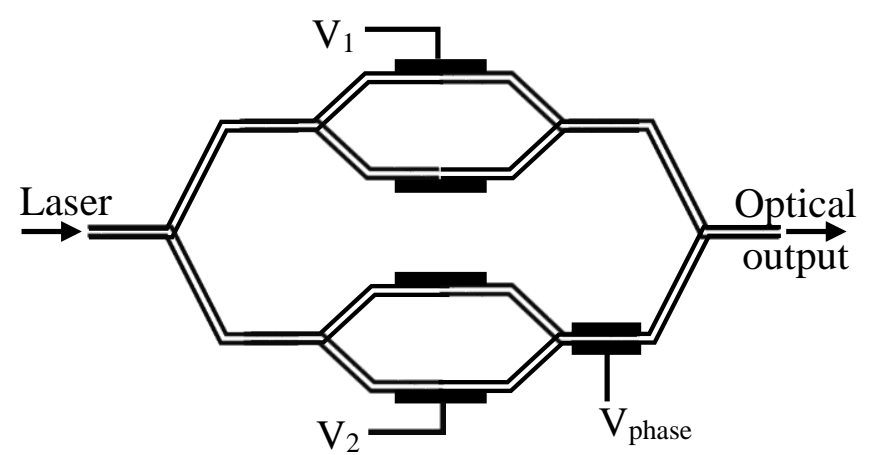

b)

Figure 2.10. Dual drive MZM (a) and nested IQ-MZM (b)

There are several variants of MZM, from a basic single-drive MZM to dual polarization IQ-MZM (IQ refers to modulators that can work for both amplitude and phase modulation). The most 
common is the dual-drive MZM. The following equation gives us the optical field at the output of the modulator, ignoring the ER of the modulator [35]:

$$
E_{\text {out }}(t)=E_{\text {laser }}(t) *\left[\frac{1}{2} * \exp \left(\frac{j \pi V_{1}(t)}{V_{\pi}}\right)+\frac{1}{2} * \exp \left(\frac{j \pi V_{2}(t)}{V_{\pi}}\right)\right]
$$

where $V_{1}$ and $V_{2}$ are the driving voltages of upper and lower arm of the modulator, $E_{\text {laser }}$ is the laser signal (see equation (12)) and $V_{\pi}$ is the biasing voltage value required to shift the phase of the optical signal by $\pi$ or 180 degrees. There are a few configurations that are common for a dualdrive MZM. One of them is to have no driving voltage for one arm. Since in this case only one driving voltage is required this means that also only one $\mathrm{MD}$ and one DAC are needed, thus reducing the costs of the transmitter. This is very advantageous for IM-DD optical transmissions since cost is the main concern here. If we assume $V_{2}=0$ then the second exponential term in (20) becomes 1, resulting in the following formula for the optical field at the out of MZM running in single-drive mode:

$$
E_{\text {out }}(t)=\frac{E_{\text {laser }}(t)}{2} *\left[\exp \left(\frac{j \pi V_{1}(t)}{V_{\pi}}\right)+1\right]
$$

The two other MZM configurations are known as push-pull and push-push. The push-pull configuration implies that $V_{1}=-V_{2}$, while in the case of push-push $V_{1}=V_{2}$. Because the absolute amplitude of the driving voltages is the same, for these two configurations a single driving voltage can be employed (just like the single-drive case). The push-pull and push-push modes also have the advantage that they provide chirp free modulation, unlike their single-drive counterpart. In order to better illustrate this, let us have a closer look at (20). If Euler's formula (see equation (22)) is applied then the result is equation (23):

$$
\begin{gathered}
e^{j x}=\cos (x)+j * \sin (x) \\
E_{\text {out }}(t)=\frac{E_{\text {laser }}(t)}{2} *\left[\cos \left(\frac{\pi V_{1}(t)}{V_{\pi}}\right)+j * \sin \left(\frac{\pi V_{1}(t)}{V_{\pi}}\right)+\cos \left(\frac{\pi V_{2}(t)}{V_{\pi}}\right)+\right. \\
\left.j * \sin \left(\frac{\pi V_{2}(t)}{V_{\pi}}\right)\right]
\end{gathered}
$$

On equation (23) the following trigonometric formula is applied:

$$
\sin (x)+\cos (x)=2 \sin \left(\frac{x+y}{2}\right) * \cos \left(\frac{x-y}{2}\right)
$$

in order to obtain:

$$
E_{\text {out }}(t)=E_{\text {laser }}(t) *\left[\cos \left(\frac{\pi\left(V_{1}-V_{2}\right)}{2 \pi}\right)\right] *\left[\cos \left(\frac{\pi\left(V_{1}+V_{2}\right)}{2 \pi}\right)+j * \sin \left(\frac{\pi\left(V_{1}+V_{2}\right)}{2 \pi}\right)\right]
$$

Finally, Euler's formula (equation (22)) is applied in (25) to get: 


$$
E_{\text {out }}(t)=E_{\text {laser }}(t) * \cos \left(\frac{\pi\left(V_{1}-V_{2}\right)}{2 V_{\pi}}\right) * \exp \left(\frac{j \pi\left(V_{1}+V_{2}\right)}{2 V_{\pi}}\right)
$$

In (26) the $\cos$ factor represents the amplitude modulation and the exponential factor represents the phase modulation. It is easy to see that if the modulator is operated in push-pull configuration then the phase modulation is 0 (thus the chirp free modulation) and if it is operated in push-push configuration then the amplitude modulation is 0 . Using the same logic as in equations (22)-(26), the equation for the single-drive configuration, (21), becomes:

$$
E_{\text {out }}(t)=E_{\text {laser }}(t) * \cos \left(\frac{\pi V_{1}(t)}{2 V_{\pi}}\right) * \exp \left(\frac{j \pi V_{1}(t)}{2 V_{\pi}}\right)
$$

In this case there is always some phase modulation of the optical carrier (i.e. chirp) and thus the push-pull configuration is preferable for IM-DD systems that employ MZMs. The push-pull configuration enables also the design of IQ MZMs. By employing two nested MZM in push-pull mode and biasing the phase shift in one of the branches by 90 degrees (the phase biased branch is the $\mathrm{Q}$ branch and the unbiased one is the $\mathrm{I}$ branch) a modulation of both intensity and phase is achieved, as illustrated in Figure 2.10(b).

An important factor that was ignored in previous calculations (for simplicity) is the ER. The same formula as in the case of the EML applies here (see equation (14)). In the case of the MZM the ER is related to the power split ratio of the optical input between the two arms of the modulator. In the ideal case this ratio should be 1 (thus the 0.5 factor for each of the exponential terms in equation (20)), resulting in an ER which goes towards infinity. However, in reality this is never the case. The variable $\alpha$ is used to quantify the effect of the ER (i.e. of the power split ratio between arms) on the optical field and it is defined as follows:

$$
\alpha=\frac{1}{2 * 10^{\frac{E R}{10}}}
$$

By introducing this new term equation (20) becomes:

$$
E_{\text {out }}(t)=E_{\text {laser }}(t) *\left[\left(\frac{1}{2}-\alpha\right) * \exp \left(\frac{j \pi V_{1}(t)}{V_{\pi}}\right)+\left(\frac{1}{2}+\alpha\right) * \exp \left(\frac{j \pi V_{2}(t)}{V_{\pi}}\right)\right]
$$

Another part that was omitted in (20) is the biasing voltage. For a basic MZM like the one in Figure 2.10(a) only one bias voltage is required, while for an IQ-MZM or a dual-polarization IQ-MZM more are needed (three and six respectively). In the case of IM-DD transmissions the basic MZM is sufficient and thus only this case in considered in the equations presented in this section.

$$
E_{\text {out }}(t)=E_{\text {laser }}(t) *\left[\left(\frac{1}{2}-\alpha\right) * \exp \left(\frac{j \pi\left(V_{1}(t)+V_{\text {bias }}\right)}{V_{\pi}}\right)+\left(\frac{1}{2}+\alpha\right) * \exp \left(\frac{j \pi\left(V_{2}(t)-V_{\text {bias }}\right)}{V_{\pi}}\right)\right]
$$

This equation is employed for simulating the MZM in all the simulation results which are presented in this thesis. In order to better illustrate how it works, equation (30) is graphically represented in 
Figure 2.11. The driving voltage is represented with green line, its position on the curve being determined by the value of $V_{\text {bias }}$. The output power is shown with red line and the red-hash area represents output levels unachievable due to ER. In IM-DD optical systems the bias of the driving voltage is usually adjusted to the quadrature point (i.e. intersection points between MZM transfer function and black dotted line in Figure 2.11). The proper driving and biasing of a MZM is not a trivial task and varies from application to application. More details about these topics are given in later chapters when discussing specific applications and experiments.

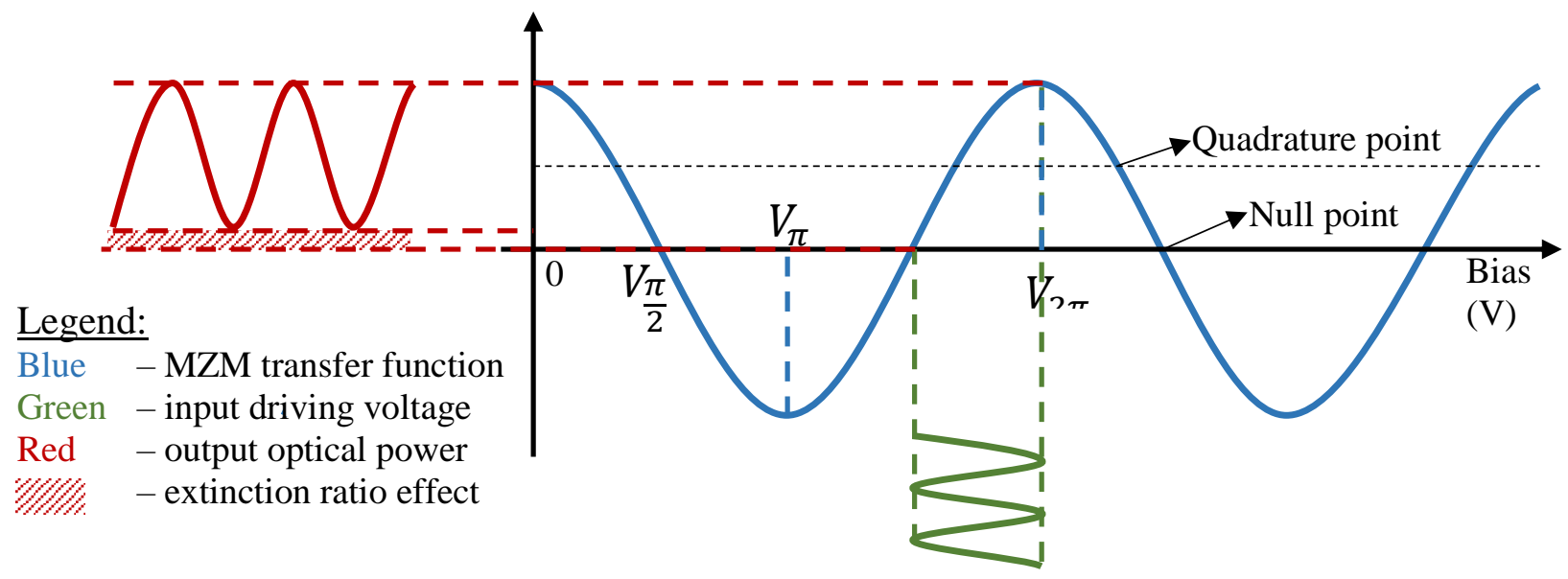

Figure 2.11. MZM transfer function

In general, MZMs can achieve better performance than EMLs in terms of bandwidth and noise. Another advantage is their high versatility, enabling modulation formats or DSP techniques that would otherwise be unavailable when using other types of modulators. In optical IM-DD transmissions longer than $10-20 \mathrm{~km}$, in the C-band, it also allows for transmitter pre-compensation of CD. The main drawback of the MZM is its high cost. Thus, in most real-life implementations of IM-DD optical systems other modulators are preferred (e.g. EML, DML). For research however MZM are very popular and they enable a wider range of techniques than the other options.

\subsection{Optical channel}

As is obvious from the previous sections, the transmission medium of choice for this work is the optical fiber. Optical networks have revolutionized the speed and range at which data are transmitted and have greatly contributed to the evolution of telecommunications. While coherent optical systems enable transmission over thousands of kilometers [36], they are expensive and DSP heavy. That is why for shorter distances of up to $80 \mathrm{~km}$ non-coherent alternatives are usually preferred. IM-DD optical systems typically employ standard single-mode fiber (SSMF) as a transmission medium. In the experiments performed for this thesis only SSMF has been used, however, there are also other types of fiber which are considered by the industry, like the multimode and multicore [37], [38] fibers. A basic overview of the most common fiber types is depicted in the following picture: 
Single-mode fiber-most common, supports two polarizations, only type of fiber used for distances larger than a few

o

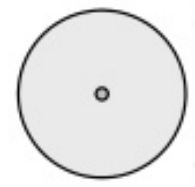

Multicore fiber - has more than one core, the cores might exhibit coupling

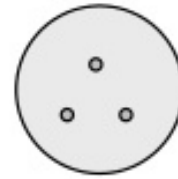

3-core

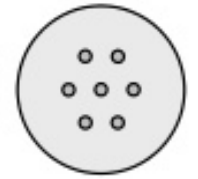

7-core

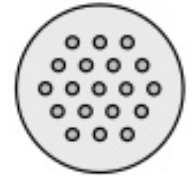

19-core
Multi-mode fiber - can support more than one spatial mode, only usable up to a few hundred meters

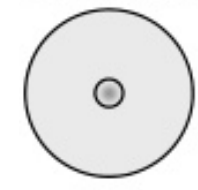

Few-mode

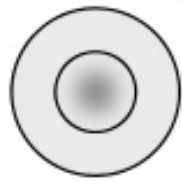

Multi-mode
Hollow-core fiber - core is made out of air, not glass like in the case of the other fibers

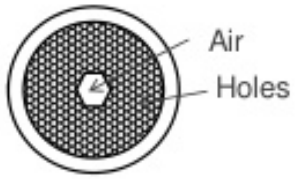

Polarization maintaining fiber - maintains linear polarization during propagation
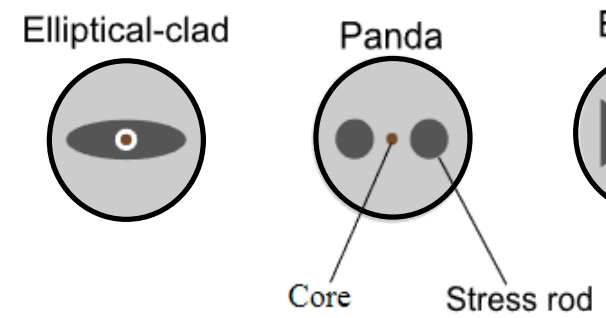

Figure 2.12. Relevant optical fiber types

When transmitting through optical fiber there are a multitude of challenges and effects that have to be overcome. They can be grouped into two main categories: linear and nonlinear. In IM-DD optical systems the linear effects are stronger. The three most critical effects, the compensation of which is an important part of this work, are fiber power attenuation, optical noise and CD (other major impairments are the bandwidth of electrical components and the electrical noise; however, these are independent of the optical domain and are not examined in this chapter). Another effect in fibers is the polarization-mode dispersion (PMD), which in long distance transmissions at high baud rates leads to portions of the transmitted signals in different polarization modes arriving at slightly different times [39] [40], thus introducing pulse broadening and ISI. In short-reach scenarios however, PMD is not too large and can be largely ignored.

The main causes of nonlinearities in fibers are the Kerr-effect (i.e. the dependence of the refractive index of the core material on power of the light [41]) and the inelastic scattering phenomenon. Since the nonlinear phenomena are more pervasive at higher optical power, they are typically less severe than the linear impairments in short-reach optical transmissions, where the received optical power is around $0 \mathrm{dBm}$ or lower. Depending on the number of channels transmitted through the optical link, the Kerr-effect can generate the following nonlinear effects: self-phase modulation, 
cross-phase modulation and four-wave mixing; while the inelastic scattering is responsible for the Raman and Brillouin scatterings. These nonlinear effects are not analyzed in more detail in this dissertation; however, more details can be found in chapter 4 of [36].

\subsubsection{Nonlinear Schrödinger equation}

The propagation of an optical pulse through a fiber can be accurately described by the so-called nonlinear Schrödinger equation (NLSE) [42]:

$$
i \frac{\partial A(L, t)}{\partial L}=-i \frac{\alpha}{2} A(L, t)+\frac{\beta_{2}}{2} \frac{\partial^{2} A(L, t)}{\partial t^{2}}-\gamma|A(L, t)|^{2} A(L, t),
$$

where $A$ is the varying amplitude of the optical pulse and $L$ is the link length (the distance travelled within the fiber at time $t$ ). On the right side of the equation the main impairments in the optical medium are represented by: $\alpha$ for the fiber attenuation, $\beta_{2}$ for dispersion and $\gamma$ for the nonlinearities within the optical fiber. The linear part of the NLSE can be extended in a Taylor series in the frequency domain, where normally only the terms up to the third order are retained [36]:

$$
\beta_{l} \approx \beta_{0}+\beta_{1}(\Delta \omega)+\frac{\beta_{2}}{2}(\Delta \omega)^{2}+\frac{\beta_{3}}{6}(\Delta \omega)^{3}
$$

where $\beta_{1}$ is the inverse of the group velocity and $\beta_{2}$ and $\beta_{3}$ are the second and third order dispersion parameters. Converting equation (32) into the time domain and adding it to the NLSE gives us a more complete formula for the propagation of a pulse in a single-mode fiber:

$$
\begin{aligned}
i \frac{\partial A(L, t)}{\partial L}=- & -i \frac{\alpha}{2} A(L, t)-i \beta_{1} \frac{\partial A}{\partial t}+\frac{\beta_{2}}{2} \frac{\partial^{2} A(L, t)}{\partial t^{2}}+i \frac{\beta_{3}}{6} \frac{\partial^{3} A}{\partial t^{3}} \\
& -\gamma|A(L, t)|^{2} A(L, t)
\end{aligned}
$$

In practice $\beta_{1}$ represents just a constant delay which does not affect the quality of the pulse and the third order dispersive effects are insignificant and thus $\beta_{3}$ can be considered as being equal to zero if the pulse is not shorter than $5 \mathrm{ps}$ and $\beta_{2}$ is not very small [36]. Taking these observations into account the NLSE (i.e. equation (31)) can be employed to evaluate the propagation of a pulse through SSMF, instead of the extended formula for pulse propagation (i.e. equation (33)).

\subsubsection{Fiber attenuation}

A pulse that is travelling through a fiber is gradually losing power. The amount of lost power scales linearly with the distance travelled through the fiber. In the NLSE the fiber attenuation coefficient is represented by $\alpha$. Its value is dependent on the wavelength of the light and it is lowest in the $\mathrm{C}$ and L-bands, that is between 1530 and $1565 \mathrm{~nm}$ and between 1565 and $1625 \mathrm{~nm}$ respectively. The dependence of the fiber attenuation coefficient on wavelength, between $1250 \mathrm{~nm}$ and $1700 \mathrm{~nm}$, is shown below, in Figure 2.13. 


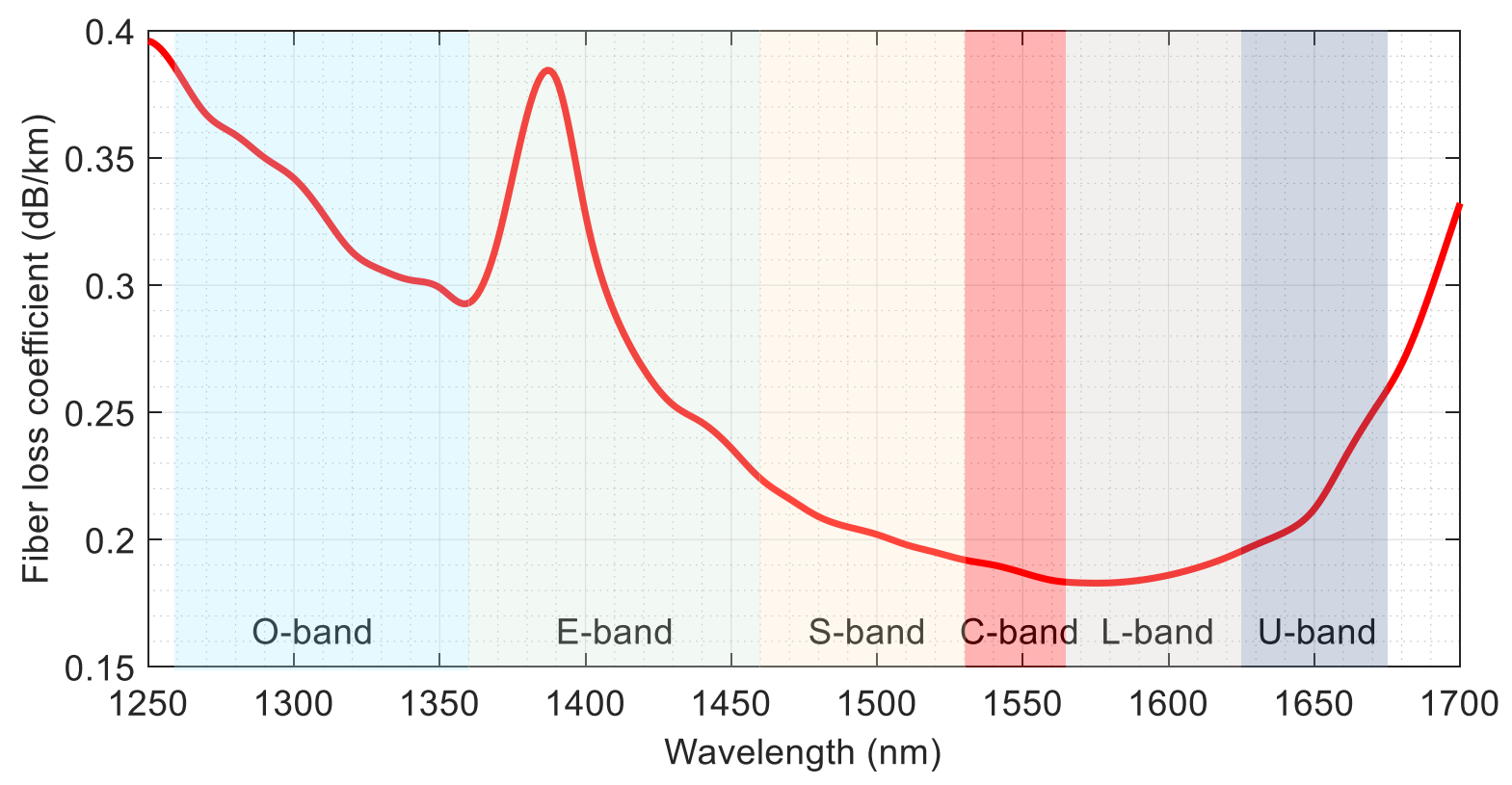

Figure 2.13. Fiber loss coefficient relative to wavelength for SSMF

The two most commonly used wavelength bands are the $\mathrm{O}$ and the C-band. In the O-band the power attenuation stands at around $0.3-0.35 \mathrm{~dB} / \mathrm{km}$ while in the C-band attenuation is below 0.2 $\mathrm{dB} / \mathrm{km}$. The typical output optical power of an EML or MZM is around $4 \mathrm{~dB}$. Since in the O-band the use of EDFA is undesirable, transmissions in this band (at high speeds of 28 GBaud or more) are usually limited to approximately $30-40 \mathrm{~km} \mathrm{[10],} \mathrm{[43].}$

\subsubsection{Optical noise and the optical signal-to-noise ratio}

The most common wavelength bands employed for optical transmissions are the O-band and the $\mathrm{C}+\mathrm{L}$ bands. For applications like intra-datacenter communications, where the maximum link lengths are of only up to a few kilometers, no optical amplification is required. However, for applications like metro-access, where transmission distances can reach or exceed $20 \mathrm{~km}$, the optical power attenuation can be too large for the receiver to properly detect the original transmitted signal. In this scenario a way to amplify the signal in the optical domain is needed. The most common type of optical amplifier used in modern optical systems is the EDFA. While the best option currently available, it is by no means a perfect device. Every time an optical signal is amplified with the help of an EDFA its OSNR is decreased. The EDFA works by doping the fiber core with erbium, which causes the erbium to be ionized. Amplification occurs if more ions are in the excited state than in the ground state. When an excited ion decays to the ground state, it emits a photon of similar energy and random phase, influencing both the phase and the amplitude of the signal [36]. This effect is referred to as spontaneous emission and it is also the source of the noise generated by EDFAs.

The NLSE described in section 2.2.1 can be updated to account for the case when EDFAs are employed in the link. When doing this, equation (31) becomes [36]: 


$$
i \frac{\partial A(L, t)}{\partial L}=i \frac{1}{2}\left(g_{0}-\alpha\right) A(L, t)+\frac{\beta_{2}}{2} \frac{\partial^{2} A(L, t)}{\partial t^{2}}-\gamma|A(L, t)|^{2} A(L, t)+i f_{n}(L, t),
$$

where $g_{0}$ is the gain coefficient and the term $f_{n}(L, t)$ represents the fluctuations induced by spontaneous emission (i.e. the noise added by the amplifier).

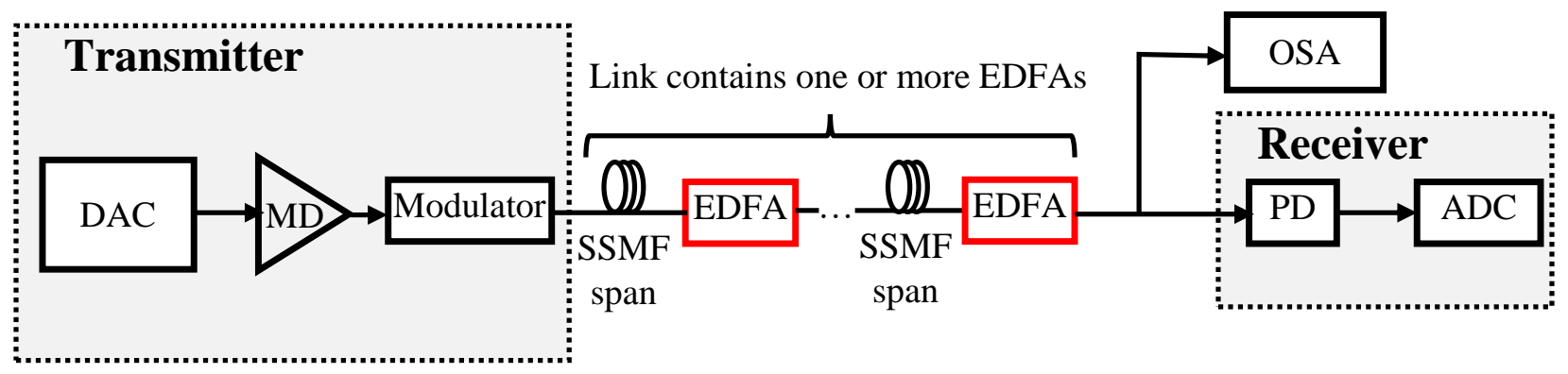

Figure 2.14. Optical transmission system employing EDFAs

If an optical transmission does not use any EDFA, then the optical noise can be ignored. In this case the performance of an optical system is usually evaluated against the received optical power sensitivity. If one or more EDFAs are present in the link, like in Figure 2.14, then the situation changes and the optical noise can become a major problem. While the classic SNR is considered in the electrical domain, in optical transmissions which employ one or more EDFAs it is useful to define the concept of OSNR. Just like regular SNR, the OSNR is the ratio between the power of the signal and the power of the noise, only this time they are measured in the optical domain instead of the electrical one.

$$
\text { OSNR }=\frac{P_{\text {signal,optical }}}{P_{\text {noise }, \text { optical }}}
$$

A way of measuring the OSNR is described next, based on the standard test method described in [44]. A signal propagating through fiber will occupy a limited bandwidth (dependent on the data rate, bandwidth of setup and wavelength). The noise on the other hand is broadband (i.e. it occupies a much larger spectrum than the signal itself). Based on these two observations we can measure the power of the noise outside the signal band and then measure the power in-band (which accounts for the signal plus the noise), around the central wavelength. Having these two values, the OSNR can be calculated as:

$$
\text { OSNR }=\frac{\left(P_{\text {inband }}-P_{\text {outband }}\right)}{P_{\text {outband }}}
$$

In experiments an optical spectrum analyzer (OSA) is employed to visualize the spectrum and to measure the required spectral powers (the whole process can be automatized in MATLAB). The signal power is measured first and then the out of band noise. Typically, the out-band noise is measured in a $12.5 \mathrm{GHz}$ band. It is also important to place the noise measuring band where the 
signal power is negligible (at least two times the signal baud rate away from the central wavelength should be enough).

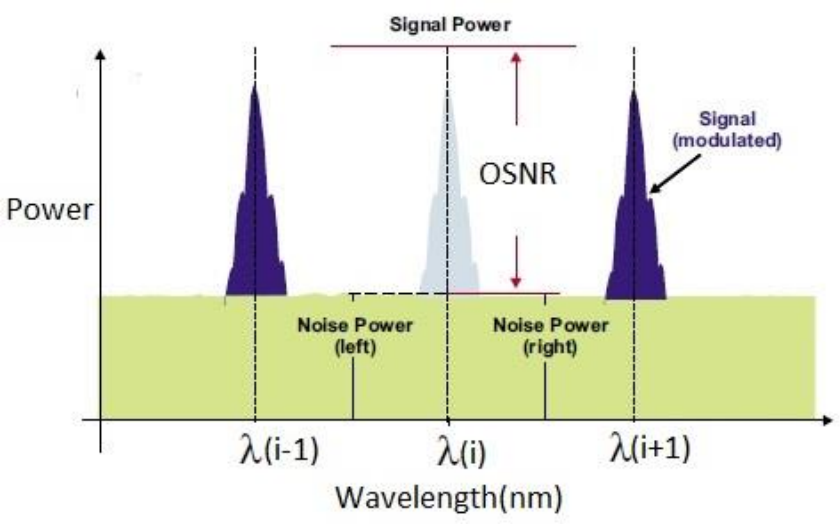

Figure 2.15. Illustration of OSNR measurement [45]. Noise power is measured on both sides of the signal spectrum

The accuracy of the measurement can be improved by performing noise measurements in outbands on both sides of the signal, as shown in Figure 2.15, or at multiple locations and then averaging the power of the noise. When $P_{\text {outband }}$ is small it might also be helpful to slightly increase the in-band when measuring $P_{\text {inband }}$. However, for most application the basic method will provide enough accuracy.

\subsubsection{Chromatic dispersion}

When propagating through a SSMF the different spectral components of an optical pulse travel at different speeds, an effect that is referred to as group-velocity dispersion (GVD). As a result of this, the spectral components arrive at the photodetector at different times, causing the received optical pulse to extend over more than one-unit interval (i.e. one period). This broadening of the pulse is known in general terms as fiber dispersion.

There are several types of dispersion which manifest in the optical fibers [46]:

- Material dispersion - as the name suggest, it is dependent on the material used to manufacture the fiber. The refractive index of these materials varies with wavelength, causing the different spectral components of an optical signal to travel at different velocities. In SSMFs material dispersion is the most dominant dispersive effect. For a graphical representation of the dependence of the material dispersion on the wavelength, see Figure 2.16.

- Waveguide dispersion - it is related to the geometry of the fiber and represents the process by which an electromagnetic signal is distorted by virtue of the dependence of the phase and group velocities on wavelength as a consequence of the geometric properties of the waveguide. The value of the waveguide dispersion can be positive or negative. For SSMF this value can be seen in Figure 2.16. 
- Modal dispersion - it occurs in multimode fibers and represents the fact that the propagation velocity of an optical signal is not the same for all modes.

- Polarization mode dispersion (PMD) - due to the asymmetries and imperfections of the SSMF the two polarizations of light, which in the ideal waveguide travel at the same speed, are propagating at different velocities.

For this work the modal dispersion and the PMD were not taken into consideration. Modal dispersion is a major concern in multimode fibers but in SSMF it can be ignored and the PMD is mostly negligible for short-reach optical transmissions. The waveguide and the material dispersion are the two components of CD.

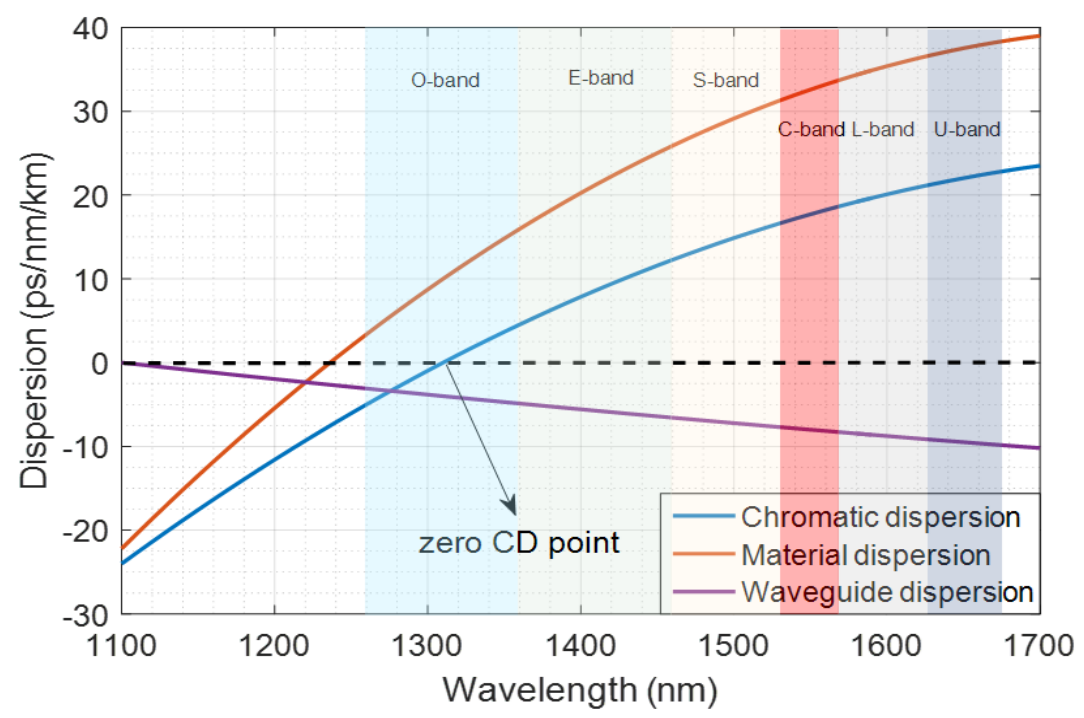

Figure 2.16. Dispersion dependence on wavelength in SSMF

CD manifests as a broadening of a transmitted pulse which will introduce ISI at the receiver if its value is large enough. The amount of $\mathrm{CD}$ is dependent on four main parameters: the dispersion coefficient of the fiber, the wavelength of the transmitted optical signal, the baud rate and the length of the transmission. For SSMF the dispersion is around $17 \mathrm{ps} / \mathrm{nm} / \mathrm{km}$ in the C-band. In the O-band this value is close to 0 and thus $\mathrm{CD}$ has almost no effect on short-reach transmissions. In the C-band, at high baud rates, $\mathrm{CD}$ becomes the main limitation when it comes to link length. Since $\mathrm{CD}$ is linear and deterministic it can be compensated for. The three most common solutions are to pre-compensate for $\mathrm{CD}$ at the transmitter side, to use a $\mathrm{CD}$ pre-compensation module or to employ CD pre-compensating optical fibers. While very large $\mathrm{CD}$ values can be compensated with these methods, in short-reach scenarios $(<80 \mathrm{~km})$ the dispersion is not large enough to justify the use of extra hardware or more expensive fibers, and in most cases the CD can be overcome by the use of the MLSE algorithm. 


\subsection{Receiver components}

In order to recover the transmitted information, the signal must be converted back into the electrical domain. This is done with the help of a photodiode. Once in electrical domain the signal is sampled by an ADC and the resulting digital data is processed in MATLAB.

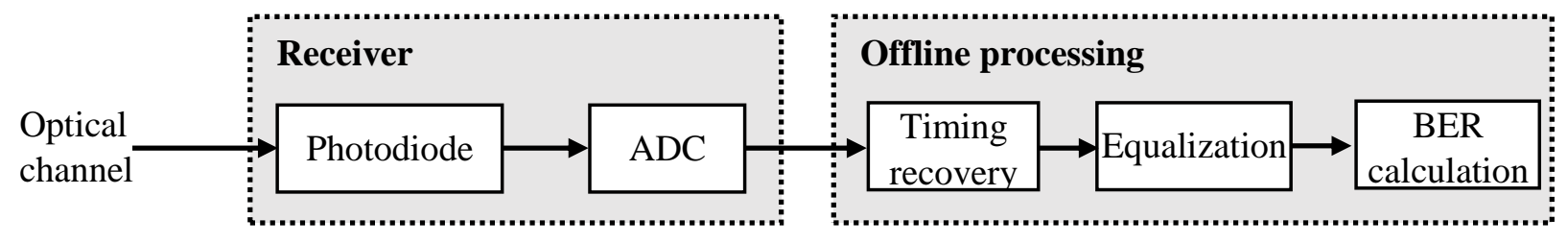

Figure 2.17. Block diagram of receiver and offline processing

In high-speed optical communications the transmitted data is not going to be error free, on the contrary, the BER of the received data is usually very high. Typically, after the data are captured timing recovery is performed first in order to obtain the best sampling phase and to mitigate the effects of timing jitter. After timing recovery equalization is performed and other DSP algorithms might be employed in order to reduce the BER. This research is focused mainly on this part and thus more details about the offline DSP are presented in the next chapters. The performance of a transmission is evaluated by calculating the BER. Optical communication systems employ some form of FEC codes to ensure a BER of 0 (i.e. BER $<10^{-15}$ ). The target of the equalization is to bring the BER below the FEC threshold (i.e. below the maximum BER value at which the chosen FEC code guarantees 0 errors at its output).

\subsubsection{Photodiode receivers}

Non-coherent receivers use $p-i-n$ photodiodes to detect the optical signal and convert it into the electrical domain. These photodiodes operate by the square law principle, meaning that they detect the power of an optical signal and output an electrical current which is proportional to the received power. The complexity of such receivers is very low (resulting in very low cost also), however they cannot recover the phase or polarization information of a signal. The three most important parameters of a non-coherent photodetector are the noise, the bandwidth and the sensitivity.

There are three main types of noise generated by a photodiode receiver: the shot noise, the dark noise and the thermal noise. The shot noise is the random fluctuation of the current generated by the photo-receiver. This fluctuation is caused by the fact that the electric current is a stream of electrons that are generated at arbitrary times. This holds true even if the received power at the input of the photodiode is constant. The current generated by the receiver can be expressed as [47]:

$$
I(t)=\sum_{n=1}^{N_{e}} q h_{c}\left(t-t_{n}\right) \cong \bar{I}+i_{s}(t),
$$


where $N_{e}$ is the total number of electrons, $q$ is the magnitude of the electron charge, $h_{c}$ gives the shape of the current produced by each received photon and $t_{n}$ is the arrival time of the $n^{\text {th }}$ photon. The equation in the middle of (37) can be expressed also as the sum of the average photocurrent, $\bar{I}$, and the fluctuating part of the current, $i_{s}(t)$, as shown in the same equation. The fluctuating part of the current is the process which generates the noise and it is a stationary random process characterized by the Poisson probability density function and white spectral power density [48]. The shot noise variance is obtained from the autocorrelation function of the square of the varying part of the current [36]:

$$
\sigma_{s}^{2}=\left\langle i_{s}^{2}(t)\right\rangle=2 \int_{0}^{f_{n}} S_{s}(f)=2 q \bar{I} f_{n},
$$

where $f_{n}$ represents the effective noise bandwidth of the receiver and $S_{s}(f)$ is the spectral density of the shot noise.

The dark noise is caused by the so-called dark current. The dark current is the current generated by the photodiode in the absence of any received light. This implies also that the dark noise is independent of the received power. It increases the more defects are present in the diode and with the photoreceiver's reverse biasing. The shot noise is a stationary random process with white spectral power density. The spectral power density of the dark current can be expressed as:

$$
S_{d}=2 q I_{d}
$$

The dark current can be added to equation (38), resulting in the following formula for the combined noise variance of the shot noise and dark noise:

$$
\sigma^{2}=2 q\left(\bar{I}+I_{d}\right) f_{n}
$$

The thermal noise is caused by the random movement of electrons inside a conductor or semiconductor. This random motion generates a fluctuating current at all times, even in the absence of any received signal. In the case of optical receivers, the main contributor to the thermal noise is the load resistor. Some photoreceivers might integrate a so-called trans-impedance amplifier (TIA), which will also add additional thermal noise. This type of noise is represented as a stationary Gaussian random process and its spectral energy can be expressed as:

$$
S_{T}=\frac{2 k_{B} T}{R}
$$

where $k_{B}$ is the Boltzmann constant, $T$ is the temperature in Kelvin degrees and $R$ is the resistance of the component that generates the thermal noise. The variance of this noise can be seen as the autocorrelation function of the current generated by the random movement of the electrons (similar to the shot noise variance): 


$$
\sigma_{T}^{2}=\left\langle i_{T}^{2}(t)\right\rangle=2 \int_{0}^{f_{T}} S_{T}(f) d f=\frac{4 k_{B} T}{R} f_{T}
$$

where $f_{T}$ represents the effective noise bandwidth and is equal to $f_{n}$ from (38). Putting equations (40) and (42) together gives us the total variance of the noise generated by the photodiode:

$$
\sigma^{2}=2 q\left(\bar{I}+I_{d}\right) * f_{n}+\frac{4 k_{B} T}{R} f_{T}
$$

The sensitivity of a photodetector is another important parameter to be considered. It can be seen as the ratio between the current generated by the photodiode and the received power. The higher the ratio the better. In simulations and experiments the sensitivity is usually defined as the minimum input power to the receiver required to achieve a BER below a pre-determined FEC threshold. The performance of an IM-DD optical transmission is often evaluated in terms of receiver sensitivity.

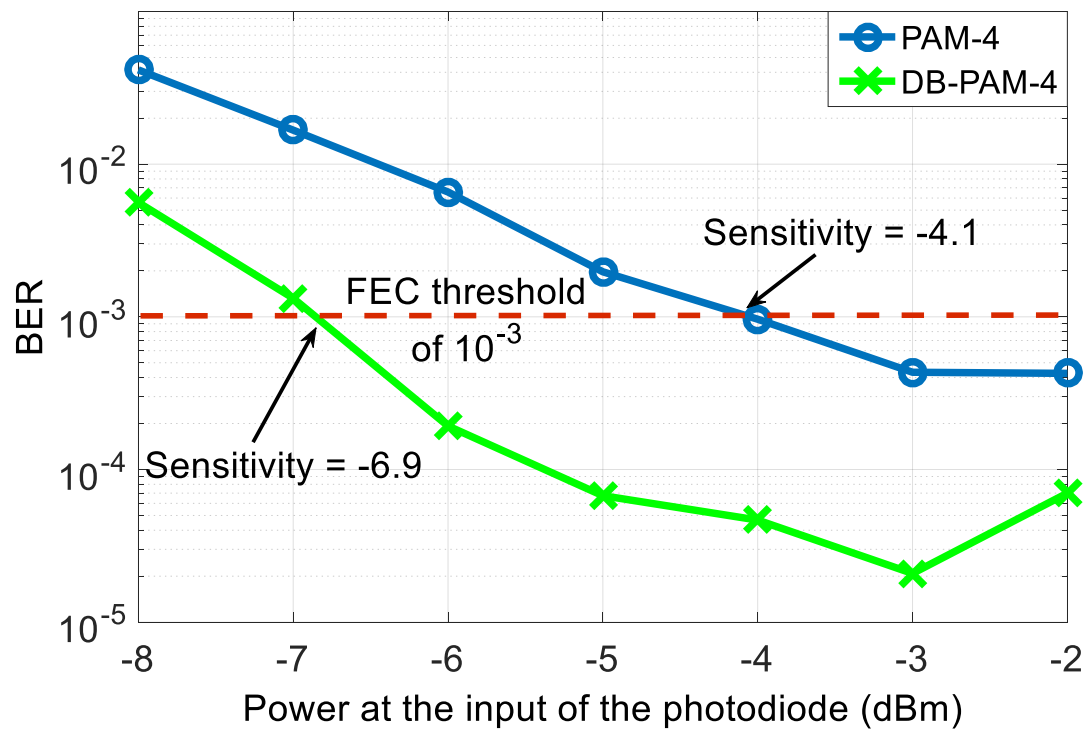

Figure 2.18. Performance of PAM-4 and DB PAM-4, 28 GBaud, BTB optical transmission illustrating the concept of receiver sensitivity

In the above figure the sensitivity of a photodiode is evaluated. First a FEC threshold is chosen, in this case $10^{-3}$. A PAM-4 and a DB PAM-4 are transmitted at a rate of 28 GBaud. The power at the input of the receiver is varied from $-8 \mathrm{dBm}$ up to $-2 \mathrm{dBm}$. In the case of PAM-4 the BER goes below the FEC threshold at around $-4.1 \mathrm{dBm}$ and in the case of DB PAM-4 at around $-6.9 \mathrm{dBm}$. Note than this definition of sensitivity is heavily influence by other factors like baud rate, transmission length and bandwidth. It is often used to characterize the performance of the whole optical system, not just that of the receiver. If we observe the BER performance of both PAM-4 and DB PAM-4 from Figure 2.18 we can see that the BER stops improving at a received power of around $-3 \mathrm{dBm}$. Further increasing the power of the optical signal will not improve the performance of the system. This is because the photodiode becomes saturated and the current it generates will 
not increase further. The sensitivity of a receiver can be thus more accurately defined as the minimum received power at which the best BER is obtained. While this value can be slightly influenced by things like modulation type and bandwidth, it is mainly influenced by the photodiode and will not vary too much depending on the optical system.

Like all other components presented in this chapter the photodetector has a limited bandwidth, bandwidth which can severely limit the performance of high-speed optical transmissions. Optical receivers employed in today's commercially available 4-lambda $100 \mathrm{G}$ optical transmission systems usually have bandwidths comparable with those of the modulator and are in the range of 10-20 GHz. More advanced photodiodes can have around $40 \mathrm{GHz}$ of bandwidth, while state of the art receivers have been demonstrated up to $100 \mathrm{GHz}$ [49].

\subsection{Experimental method}

Now that the most important components of an IM-DD optical transmission system have been introduced, we can discuss the basic experimental workflow. The main goal of this section is to offer an overview of the experimental method which was applied to all of the experiments presented in this thesis. While for each experimental scenario the best suited components might be different, the roles they serve stay more or less the same, allowing us to give a generalized view of the experimental setup. For example, in all setups the data at the transmitter side has to be converted from digital to analog, however sometimes a BPG might be enough to do this (when no DSP is required at the transmitter) and other times a DAC or AWG are necessary. Below, in Figure 2.19 , the experimental setup template is illustrated.

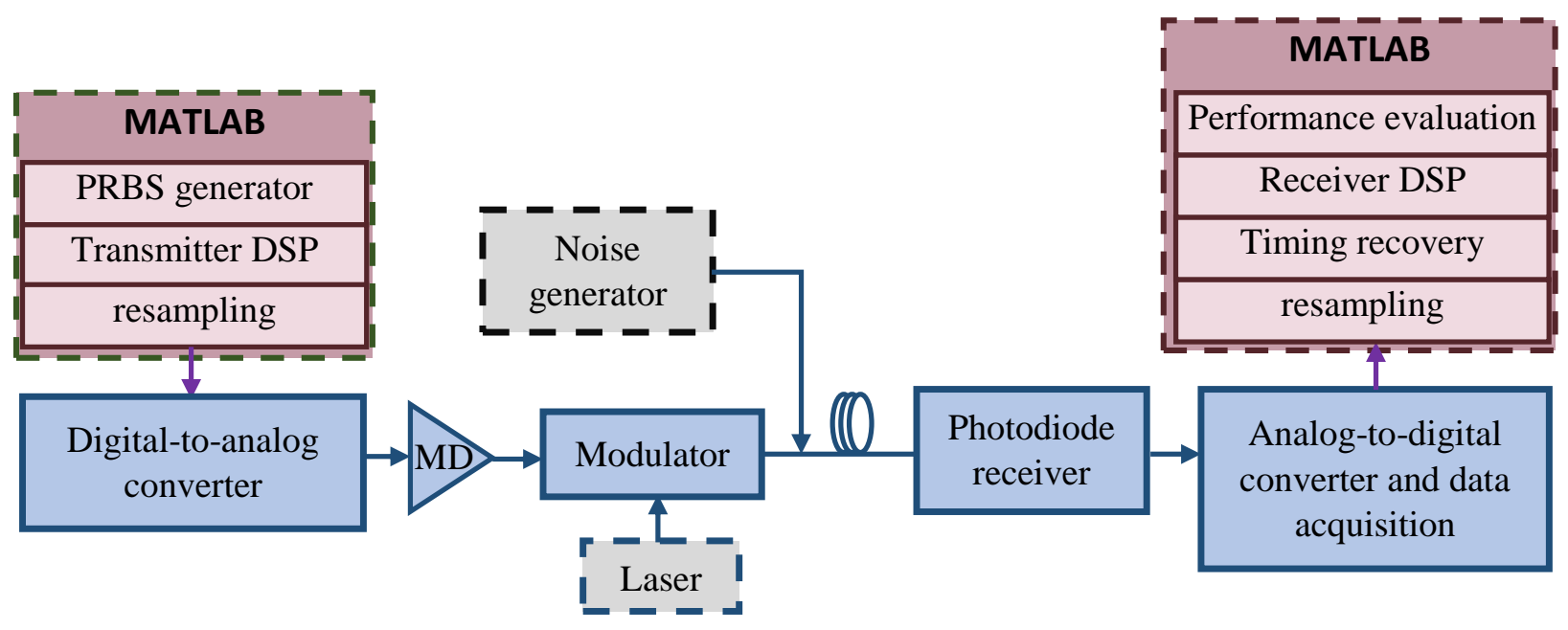

Figure 2.19. Generalized experimental setup block diagram

Before an experimental setup can be built there are a few steps to consider. The first step is to determine which optical transmission scenario needs to be investigated and to determine which impairments are dominant in said scenario. For example, if we want to achieve a BTB, 112 Gbps PAM-4 transmission then, gwiven the high required baud rate, the bandwidth of the components 
and the ISI introduced by them would be the biggest concern, while if the target is a $40 \mathrm{~km}, 56$ Gbps PAM-4 transmission the dominant impairment would be CD. Once the transmission scenario is set, we can start designing DSP algorithms, which would first be tested in MATLAB simulations. If their performance in simulations is deemed satisfactory then they need to be tested with an experimental setup.

Before building the physical experimental setup, the signal corresponding to chosen scenario has to be created in the digital domain. In this thesis MATLAB was used for this purpose. We begin by generating a PRBS. Next follows the transmitter DSP algorithms which involve the generation of the chosen modulation (e.g. PAM-4, PAM-8, TCM) and the implementation of any DSP algorithms that have to be applied at the receiver side (e.g. digital pre-emphasis, constellation shaping). Next step is to resample the signal to the sample rate required by the DAC in order to achieve the targeted transmission baud rate (see more details in section 2.1.1), and to convert the digital signal into the analog domain. However, if a BPG is employed then no transmitter DSP is required (or possible for that matter). In this thesis the BPG was employer only once, see section 4.3.2. The amplitude of the signal at the output of the digital-to-analog converter (be it DAC, AWG or BPG) is never high enough to properly drive the modulator, thus a MD is required. Next the signal is sent to the optical modulator. In the experiments presented in this work EMLs and MZMs were employed. In the case of EML a bias-T is also needed before the modulator to adjust the signal bias, while in the case of the MZM an external CW laser source is required. The modulator converts the electrical signal into an optical signal. The optical link consists of SSMF fiber with length ranging from 0 to $80 \mathrm{~km}$. In the case an optical noise mitigation DSP algorithm is tested, a noise source is required in order to control the amount of optical noise in the link. In the experiments presented here an EDFA is fulfilling this role. The optical signal is converted back into the electrical domain by a PD. In some experimental setups presented in this thesis the PD is integrated together with a TIA, in which case the receiver is referred to as a receiver optical subassembly (ROSA). The signal has to be converted back to digital after which data acquisition takes place. Both of these functionalities are fulfilled by a real-time oscilloscope. The acquired data, referred to here as offline data, is then processed in MATLAB. It is resampled, usually to 2 samples/symbol, and the timing recovery is performed. Next the data is processed by the receiver DSP. While for some scenarios the tested DSP algorithm might be placed at the transmitter side, in order to achieve low enough BERs in high-speed optical transmissions some sort of transmitter side equalization is always required. The final step in the experimental workflow is to evaluate the performance. The metric used for this purpose is the BER. The target is to bring the BER below a threshold which is determined by the FEC used by the optical system. The BER is calculated at certain OSNR values (usually when noise mitigation algorithms are evaluated) or at different received power values. From these BER versus OSNR or BER versus received power curves the minimum OSNR or received power at which the BER goes below the FEC threshold is calculated, referred to in this work as noise sensitivity or received power sensitivity, respectively. 


\section{Chapter 3}

\section{Receiver digital signal processing}

From a business point of view the goal of non-coherent optical transmission systems is to ensure the lowest cost possible for short-reach networks. While coherent systems outperform IM-DD systems and they enable very long transmission distances, they cannot yet compete in price in the short-reach market. Future technology advancements might enable cheaper coherent systems but right now and for the short-term future non-coherent is the preferred solution for cost-effective transmissions systems with ranges under $80 \mathrm{~km}$ (although coherent devices for short-reach already exist [50]). Since cost is the number one consideration, IM-DD optical systems do not use the most expensive components and push transmission rates as high as possible, resulting in a heavily degraded signal at the receiver. All non-coherent systems have some form of FEC in order to ensure BERs below $10^{-15}$ (which is considered error free). However, since FEC algorithms can be very complex, some compromises must be made between complexity and error correcting capabilities. This implies that the FEC BER thresholds (the maximum BER at the input of the FEC block that guarantees error free FEC output) in IM-DD optical systems are not very high. In contemporary high speed non-coherent transmissions the received data is heavily deteriorated and the resulting BER is typically above the FEC threshold. To bring the BER below the FEC threshold DSP is necessary and thus it is an essential part of all modern non-coherent optical transceivers.

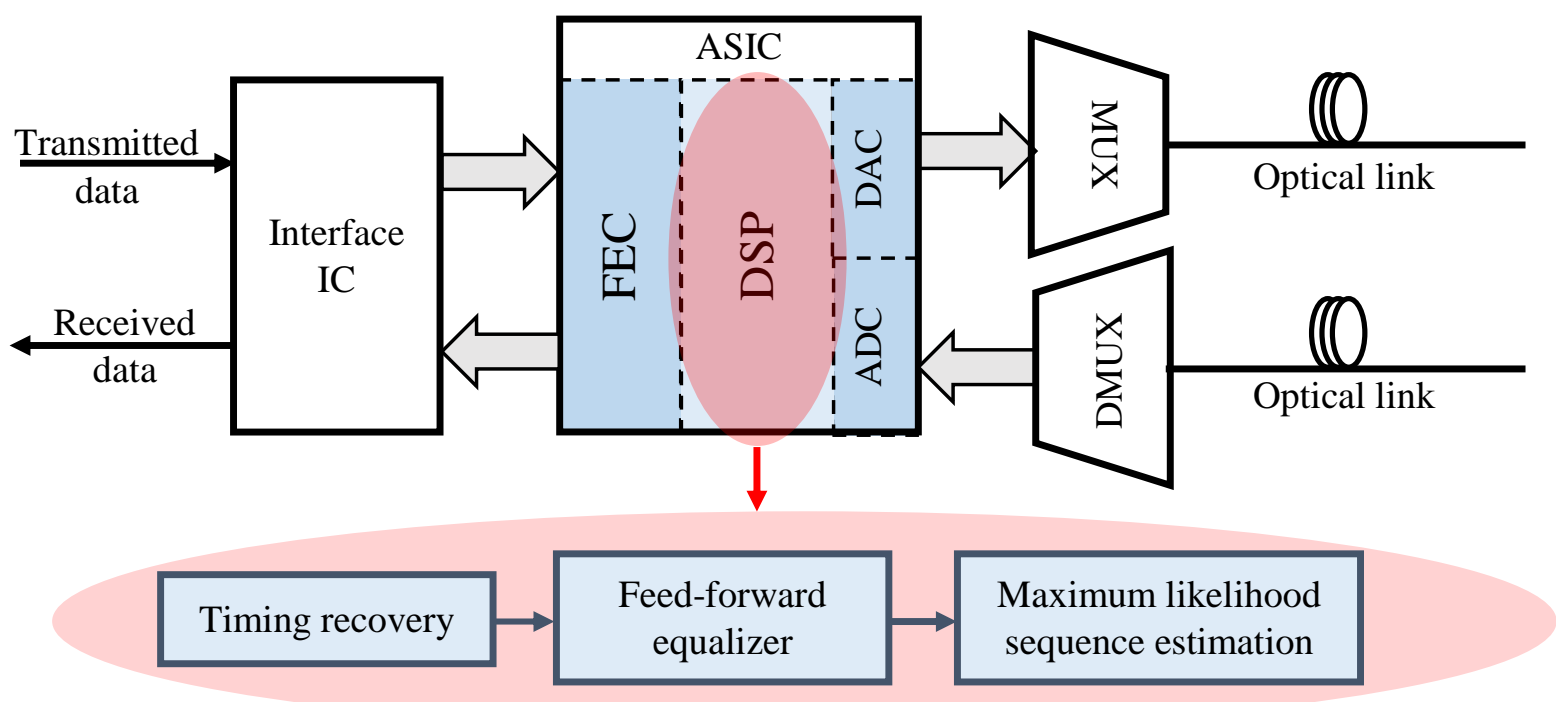

Figure 3.1. CFP optical module and the most commonly used DSP blocks 
While most of this thesis is dedicated to DSP techniques which are employed in IM-DD systems, in this chapter we focus on some of the most widely spread algorithms: the FFE and the MLSE. Timing recovery is also a crucial part of these systems; however, it was outside the scope of this thesis and is not analyzed in detail here. For the experiments performed during this thesis the Mueller and Müller [51] and Gardner [52] timing recovery algorithms were employed. More details on timing recovery can be found in [53], [54].

\subsection{Feed-forward equalizer}

The FFE is the most common piece of DSP employed in IM-DD optical systems. Its main purpose is to compensate at the receiver side the transfer function of the whole system. The non-coherent systems can be interpreted as linear time-invariant discrete (LTID) systems. If $x$ [n] is the transmitted data and $y[n]$ is the received data the LTID can be expressed as [55]:

$$
y[n]=-\sum_{k=1}^{K} u_{k} y[n+K-k]+\sum_{l=0}^{L} w_{l} x[n+L-l]
$$

where $u_{k}$ are the feedback coefficients and $w_{l}$ are the feed-forward coefficients. If at least one of the feedback coefficients is non-zero then the system has an infinite impulse response (IIR). If all feedback coefficients are equal to zero then the system has a finite impulse response (FIR). The FFE is in essence a FIR filter. There exist also IIR filter designs, referred to as decision feedback equalizers (DFE). While they can bring some gain to an optical system [56], the use of DFEs is not as widespread as that of FFE due to error propagation and instability, and thus are not presented in detail here. The block diagrams of a FIR filter and an IIR filter are shown in the figure below.

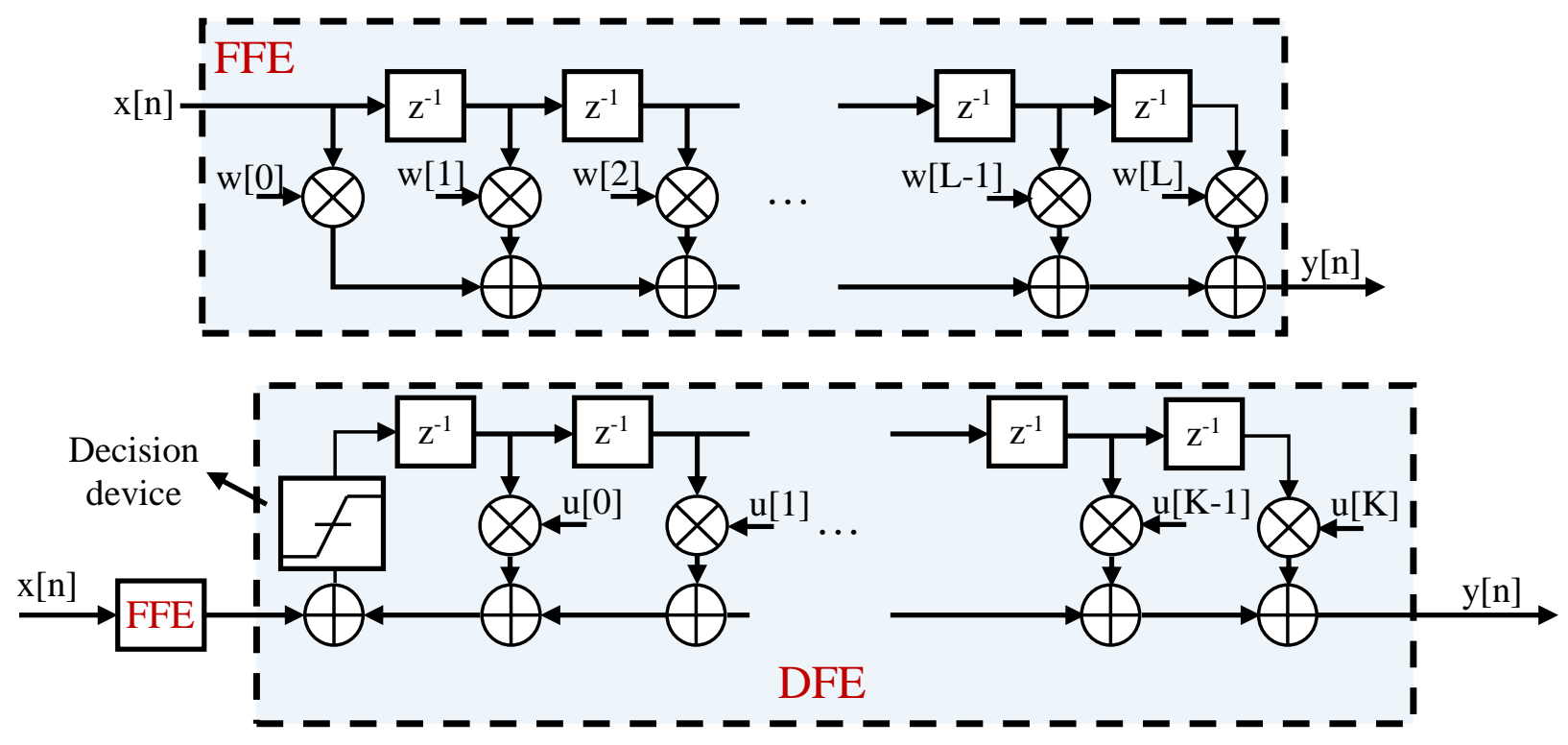

Figure 3.2. Basic structure of a FFE filter (top) and a DFE filter (bottom) 
In Figure 3.2 a time domain variant of the FFE is shown. In frequency domain the FFE tries to inverse the transfer function of a transceiver while in time domain it corrects the pulse response. Usually in IM-DD systems the FFE is designed in the time domain. There are three main parameters to consider when building such a filter. One is the number of coefficients (more commonly referred to as taps) of the filter, $L$, another one is the value for each of the coefficients or taps and the last one is the spacing between taps.

The number of taps required to properly compensate for a transmission varies greatly from case to case. The taps can be split into two categories: post-cursors and pre-cursors. If we refer to the sample that is filtered at a certain time as the central tap (tap number 0 ) then post-cursors are the taps after the central tap (positive numbered taps) and pre-cursors are the taps before the central tap (negative numbered taps). The post-cursors compensate for the influence of future samples on the current sample and the pre-cursors compensate for the influence of past samples. The bandwidth of the system is one major factor when considering this number. The higher the ratio between the baud rate of the data and the $3-\mathrm{dB}$ bandwidth, the higher the number of taps required.

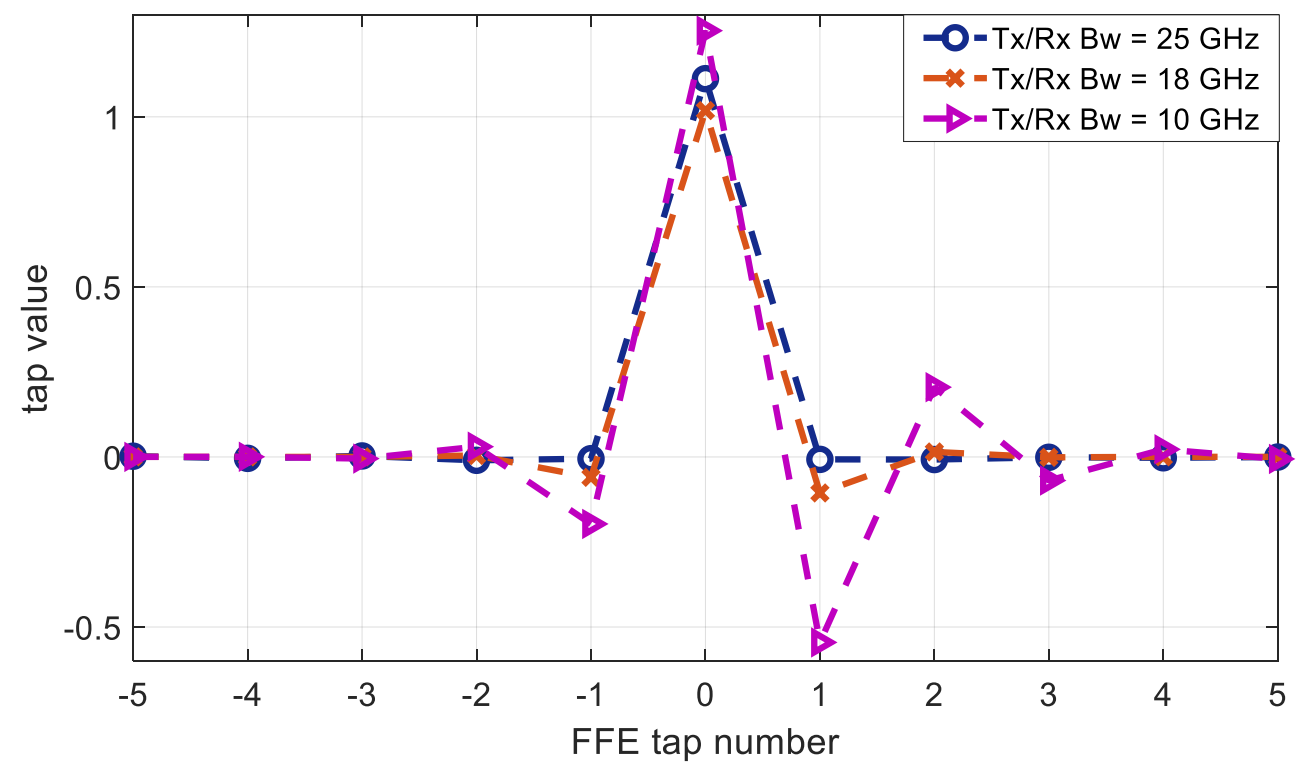

Figure 3.3. The tap values for an 11-tap FIR filter for a 28 GBaud, PAM-4 BTB transmission (simulation). Pre-cursors are denoted with negative numbers and post-cursors with positive numbers. The bandwidths of the transmitter and of the receiver are varied to 25,18 and $10 \mathrm{GHz}$

To better illustrate this fact a simulation was performed where an 11-tap FIR filter is implemented. The bandwidth of the transmitter and of the receiver are also simulated. The goal is to see how variations in bandwidth affect the tap values and the results are displayed in Figure 3.3. If a 28 Gbaud PAM-4 signal is employed and the bandwidth of the transceiver is set to $25 \mathrm{GHz}$ then there are almost no bandwidth limitations in the system and thus the values of the FFE taps are practically 0 (excepting the central tap of course). When lowering the bandwidth to $18 \mathrm{GHz}$ the values of the first pre- and post-cursors are no longer 0 . This means that the ISI will affect the two 
neighbors of a symbol. Lowering the bandwidth further down to $10 \mathrm{GHz}$ increases the required FFE taps to six (two pre-cursors and four post-cursors).

In most practical implementations 21 taps or less are sufficient to compensate for the bandwidth limitations of the transmission system. However, there are other phenomena which can influence the required number of taps, such as the presence of electrical reflections and/or CD. The higher the symbol rate of the transmission, the more severe are the effects of such impairments. In Figure 3.4 the effects of very limited bandwidth and of reflections can be observed in a very high-speed scenario (i.e. 90 GBaud PAM-4). In this case the overall 3-dB bandwidth of the experimental setup is more than four times lower than the baud rate. As a result, around 12 post-cursor taps and 7-8 pre-cursor taps are required to compensate for the bandwidth limitations. However, if we were to use a 21-tap FFE the performance after the equalizer would not be so good. This is due to reflections caused by imperfect connections in the system. In order to improve the quality of the received signal more pre-cursor taps have to be added to mitigate the effects of said reflections. CD leads to a broadening of the transmitted optical pulse and introduces ISI in the system. While in the context of short-reach optical transmissions it is rare that the ISI introduce by CD affects more than a few symbols around the transmitted one, its effects are much stronger than in the case of bandwidth limitations or reflections. As a result FFEs will struggle to mitigate its influence and in the case of extreme dispersion the training of the FIR equalizer can fail and destroy performance. There are other DSP techniques that are much more effective at combating CD, some of which will be introduced later in this work.

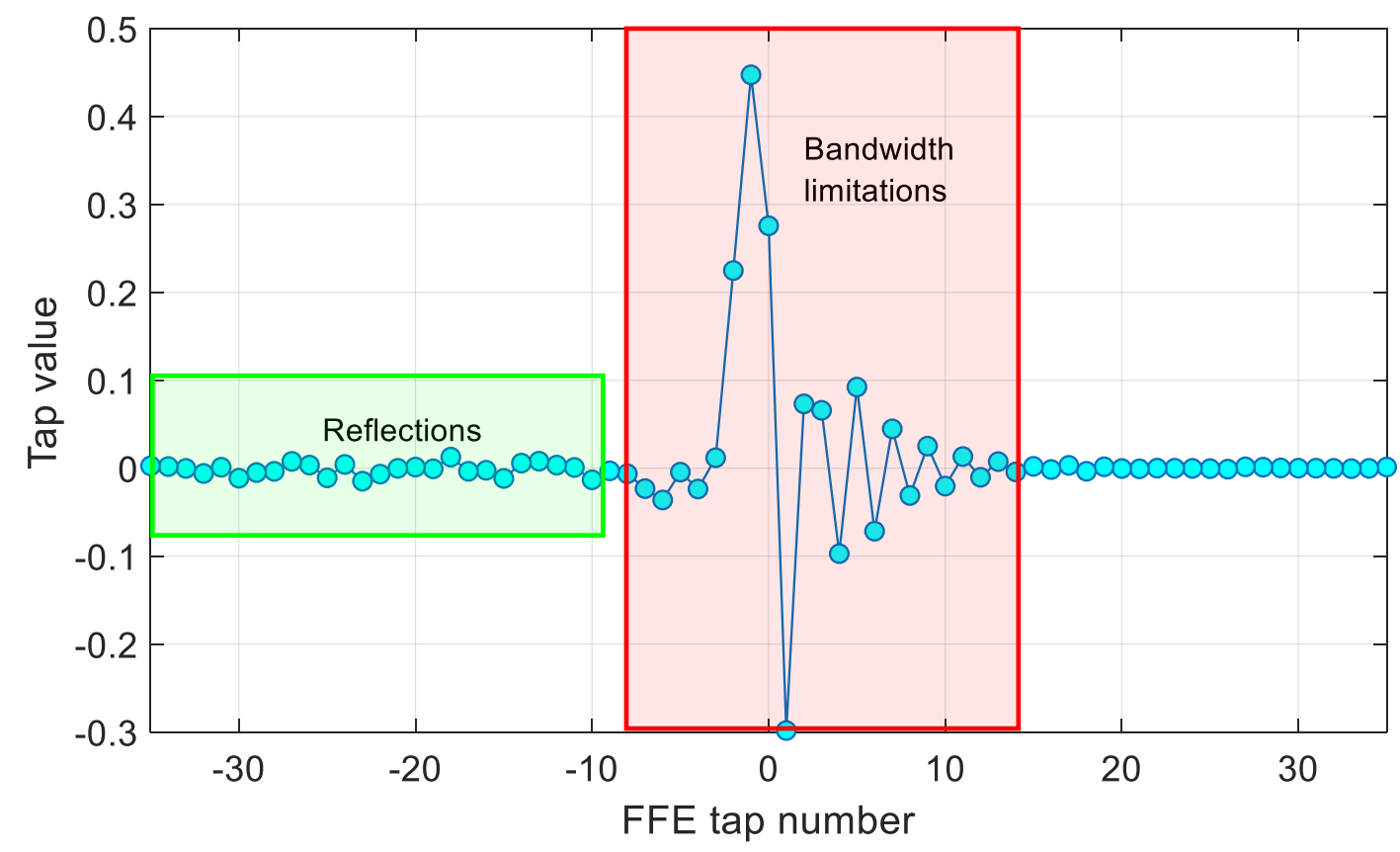

Figure 3.4. The influence of bandwidth limitations and reflections on the tap values of a 71-tap FFE for a high speed 90 GBaud PAM-4 BTB optical transmission 
In all of the examples presented in this section the spacing between the filter taps is equal to $T$ (representing the period of the signal, $\frac{1}{\text { Baud rate }}$ ). This is true also for most of the experimental results which are presented in later chapters. In real-life high-speed IM-DD optical systems the filter taps spacing is also often equal to $T$ or in some cases it is equal to $\left(T * \frac{A D C_{\text {sampling rate }}}{\text { Baud rate }}\right)^{-1}$. While having the taps closer together might improve performance in some scenarios, it comes at the price of increased complexity. If, for example, we were to design a FFE with $\frac{T}{2}$ spacing between taps the filter would require twice the number of taps as a $T$ space filter in order to compensate for the same impulse response. In the majority of cases the gain provided by decreasing the spacing filter taps spacing is too small to justify the increase in complexity.

The main part of designing a FFE filter is setting the tap coefficient values. Since these values are highly dependent on the channel and the system, they will vary a lot from scenario to scenario. Thus, some algorithm for calculating the value of the taps is needed. There are two major approaches to this problem:

- Training mode, where the transmitted data is assumed to be known or partially known.

- Adaptive blind mode, where adaptive refers to the fact that the values of the taps are continuously adjusted and blind refers to the fact that the transmitted symbols are unknown.

Both modes employ the least mean squared (LMS) algorithm to calculate the taps. This algorithm is adapted for all the modulation formats which are presented in this work and all the tap coefficients employed in the processing of simulation or offline data are obtained via LMS. The version of LMS detailed here employs the mean-square error (MSE) criterion, which implies adjusting the tap coefficients of the equalizer such that the value of the MSE is minimized.

In order to explain the LMS algorithm let us first consider just the training mode. The notations from Figure 3.2 are used, where $x[n]$ is the $n^{\text {th }}$ transmitted symbol, $y[n]$ is the estimate of the same symbol at the output of the FFE, $L$ is the number of linear feed-forward taps and $w$ are the tap coefficients. The goal of the LMS algorithm is to determine, based on the received data or a training sequence, the optimum tap coefficients. The first step is to initialize the tap values. There are two main ways to approach the initialization problem. If nothing is known about the channel then the starting point is assumed to be the ideal channel, meaning that all the taps coefficients are 0 except a single one which is set to 1 . The tap coefficient that is initialized to 1 corresponds to the current symbol and is referred to as the central tap. For example, if we have an 11-tap FFE and we would want the central tap to represent the currently transmitted symbol and have five pre and five post-cursors then tap number six would be initialized to 1 and the rest to 0 . If the transfer function of the system is known or partially known, then the tap coefficients can be initialized according to this transfer function. In real life optical systems the taps of the FFE are initialized according to a known channel model. Since the optical channel is relatively stable, if the channel model is accurate to begin with, then no adaptive training of the taps is required. However, in the 
experiments presented in this work, the channel is assumed unknown and the tap values are initialized to 1 for the current symbol tap and 0 for the rest.
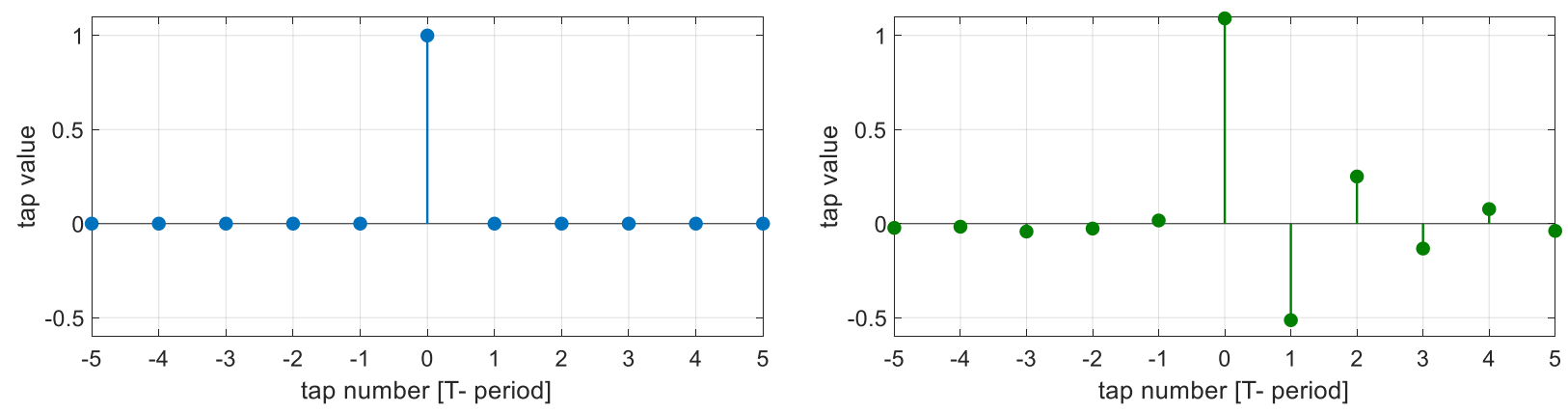

Figure 3.5. Initial tap value when the system transfer function is unknown (left) and for a specific known system (right). The values on the right side will always vary depending on the system

After the tap's coefficient have been initialized the training process can start. The first step is to pass the received sample through the FFE. The output of the filter can be expressed as:

$$
y[n]=\sum_{l=0}^{L} w_{l} x[n+L-l]
$$

The MSE criterion which is used to calculate the error is defined as [19]:

$$
\varepsilon[n]=\bar{y}[n]-y[n],
$$

where $y[n]$ is the $\mathrm{n}^{\text {th }}$ output of the equalizer, $\bar{y}[n]$ is the $\mathrm{n}^{\text {th }}$ transmitted information symbols and $\varepsilon[n]$ is the error value. In training mode $\bar{y}$ is exactly the transmitted symbols. After the error is calculated, the next step is to update the tap coefficient values:

$$
\left(\begin{array}{c}
w_{0}[n+1] \\
w_{1}[n+1] \\
\vdots \\
w_{L}[n+1]
\end{array}\right)=\left(\begin{array}{c}
w_{0}[n] \\
w_{1}[n] \\
\vdots \\
w_{L}[n]
\end{array}\right)+\mu \varepsilon[n] *\left(\begin{array}{c}
x[n] \\
x[n+1] \\
\vdots \\
x[n+L]
\end{array}\right),
$$

where $\mu$ is the gradient step coefficient. This coefficient controls the speed with which the tap values will converge. Its optimum value varies from scenario to scenario. When dealing with a fast time variant channel the value of $\mu$ should be set high (typically 0.1 to 0.01 ). However, in the case of high-speed optical systems the channel is more or less stable, and thus the gradient step coefficient can be set to lower values (e.g. 0.001 to 0.0001 ). If $\mu$ is very high (around 0.01 to 1 ) then the LMS algorithm will converge very fast but it might fail to properly converge, locking to a false local minimum, or it might not even converge at all. In Figure 3.6 it can be seen that the tap coefficient values change faster in the beginning of the training but as they approach the optimum values the speed with which they vary decreases. As a result $\mu$ can also vary in time in order to optimize the convergence speed - at the start of the training process it can be set to higher values 
(in most of the experiments performed for this thesis $\mu$ can start at around 0.01) and as the error differential $\Delta \varepsilon=\operatorname{abs}(\varepsilon[n+1]-\varepsilon[n])$ drops, so should the value of $\mu$ decrease (typically down to 0.0001). The new tap coefficients from (47) replace the old ones in equation (45) and the algorithm (i.e. equations (45) to (47)) repeats again for a new sample. If the system is time-variant then the LMS training can run for the entire duration of the transmission. When the transmission system is time invariant then the training can be stopped once the value of $\varepsilon$ drops below a certain threshold (which should be very close to 0 ). In Figure 3.6 a few examples of how the value of $\mu$ influences the LMS training convergence are given (for an 11-tap FFE) for a PAM-4, 56 GBaud BTB transmission. When the value of $\mu$ is too high (i.e. $\mu=0.01$, top left subplot) the tap coefficients fail to properly converge. If $\mu$ is reduced by a factor of 10 (top right subplot) then the coefficients properly converge and the training is successful. If $\mu$ is further reduced by a factor 10 down to 0.0001 (bottom left subplot) then the convergence is very slow and the training sequence will not be long enough for the tap values to stabilize. The convergence process can be accelerated by starting with a high value for $\mu$ and gradually decreasing it value (bottom right subplot).
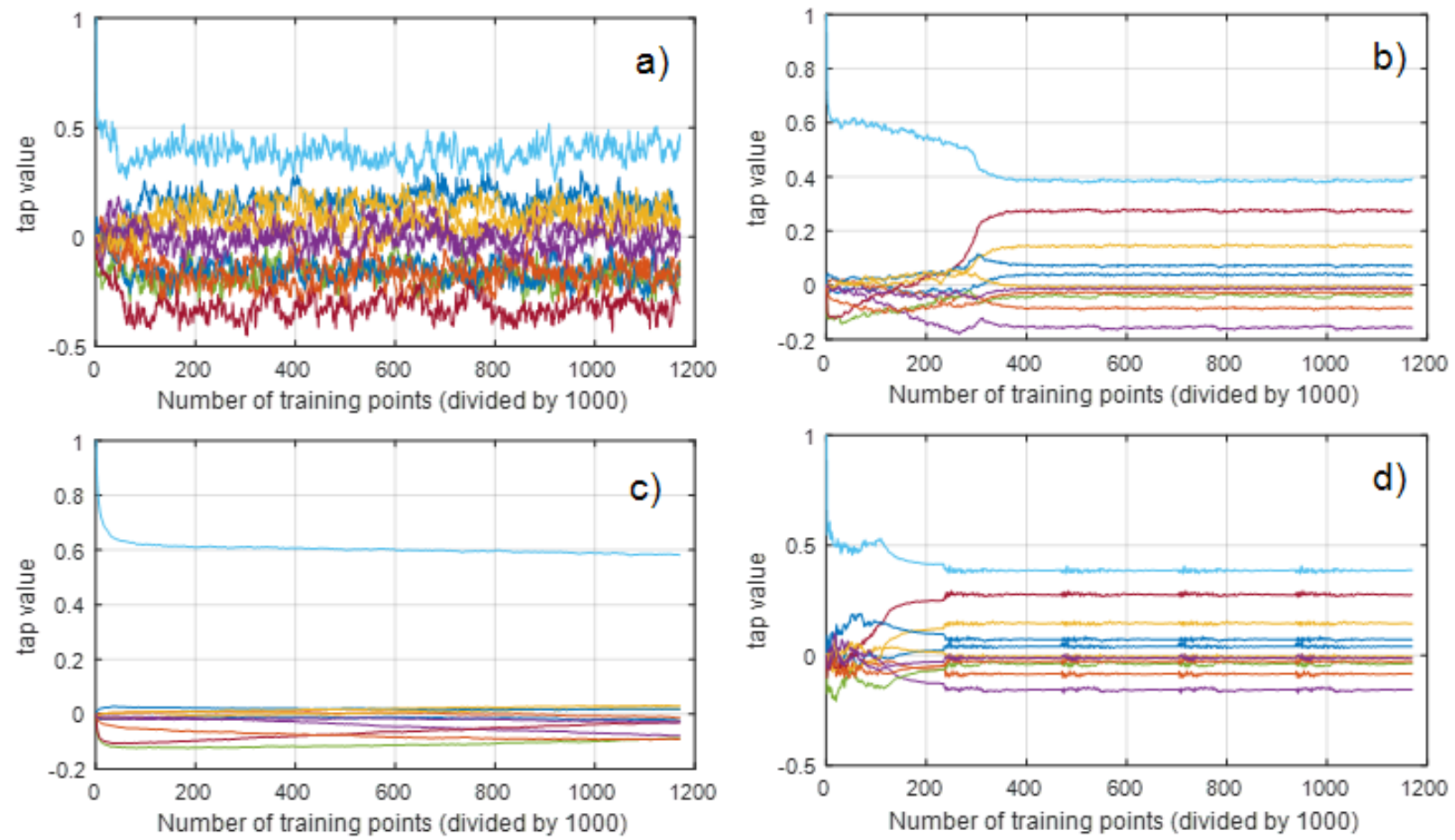

Figure 3.6. The influence of $\mu$ on tap coefficients convergence for $\mu=0.01(a), \mu=0.001(b), \mu=0.0001$ (c) and $\mu=0.01$ to 0.0001 (d)

In the case of a blind equalizer the same LMS algorithm can be used, with the only difference being that $\bar{y}$ from the MSE criterion (equation (46)) is no longer the known training sequence but instead represents the decision on the output of the FFE, meaning the modulation level closest to the value of the FFE output (e.g. if PAM-4 is transmitted with levels $0,1,2$ and 3 and the ${ }^{\text {th }}$ output of the FFE is 1.7 then the $n^{\text {th }}$ decision on the output of the FFE is 2). In the case of optical systems with high ISI many of the decisions made during the blind training will be incorrect resulting in 
higher values for $\varepsilon$. This results in slower and less accurate convergence of the tap coefficients and in extreme cases even in the failure of the training. A block diagram overview of the FFE LMS algorithm, including both training and blind modes, is shown in Figure 3.7.

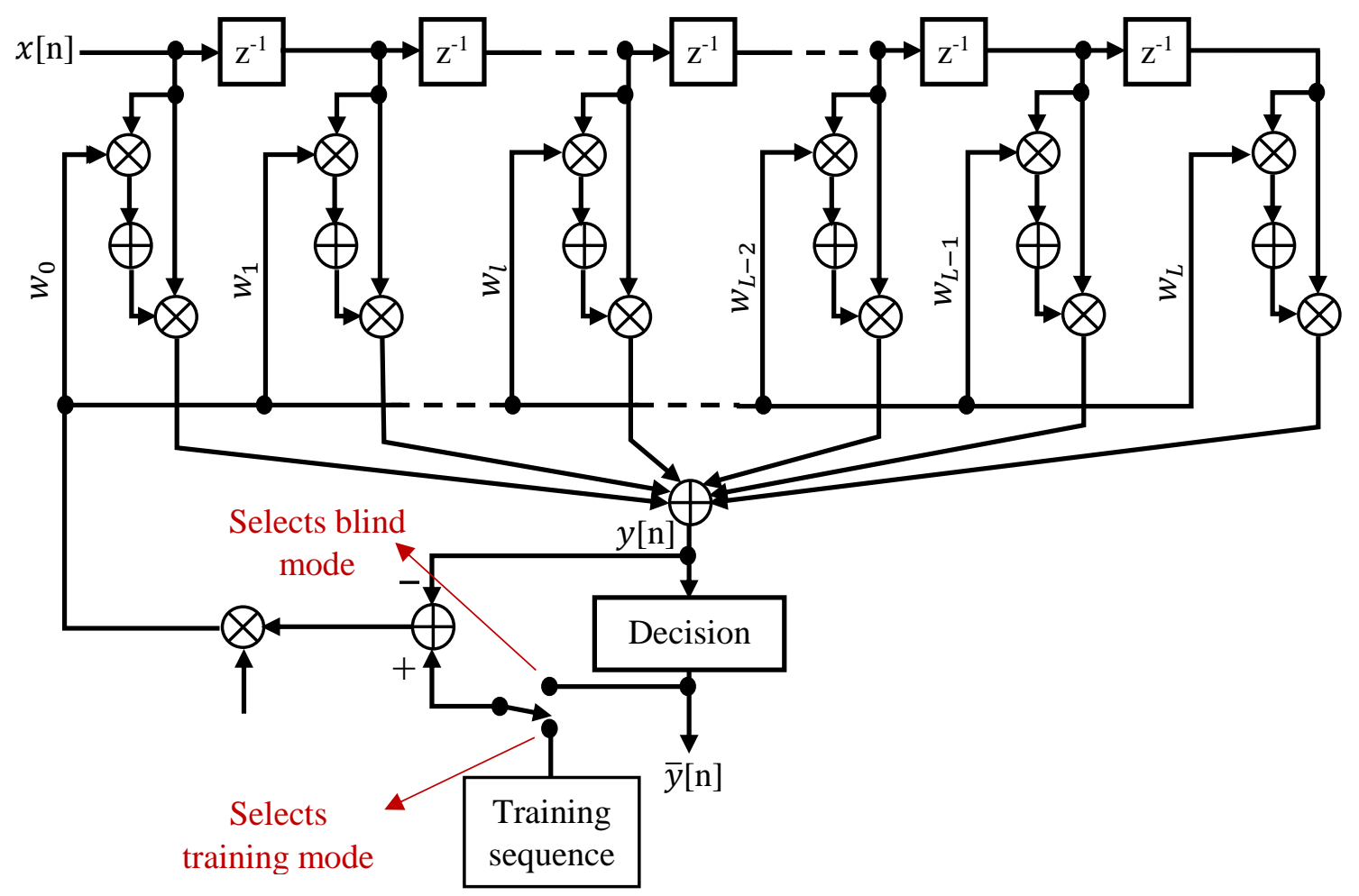

Figure 3.7. Block diagram of FFE LMS training

The FFE is employed in almost all of the experimental work done during this thesis. The only scenario where FFE is sometimes bypassed is when the CD values in the link are high. Since the experimental performance of the FIR equalizer is evaluated many times in the following chapters, a more in-depth analysis of the FFE's capabilities is not presented in this chapter.

\subsection{Maximum likelihood sequence estimation}

When the bandwidth limitations or the CD influence on the IM-DD optical transmission become too high the FFE struggles to accurately detect the transmitted signal. In these cases a better detection algorithm is required. This is where the MLSE comes into play. The MLSE is the optimum receiver DSP in terms of performance and guarantees the best detection. Its main drawback is its very high implementation complexity, which grows exponentially with the number of taps, unlike the FFE which has a linear increase in complexity with the number of taps. Deciding whether to use FFE, MLSE or a combination of both is a case by case decision. In later chapters (i.e. chapter 4 and 5) all of these three options will be explored experimentally. 


\subsubsection{The Viterbi algorithm}

The MLSE equalizer uses the Viterbi algorithm in order to detect the most likely transmitted symbol at any given time. The Viterbi algorithm gets its name from Andrew Viterbi who first introduced it in 1967 as a decoder for convolutional codes [57]. Shortly after it was adapted for use as a detection algorithm in transmission system affected by ISI [58], [59], [60]. The Viterbi algorithm works by looking at all the possible transmitted sequences and, based on a model of the channel and the received data, dec ide which of the sequences is the most probable.

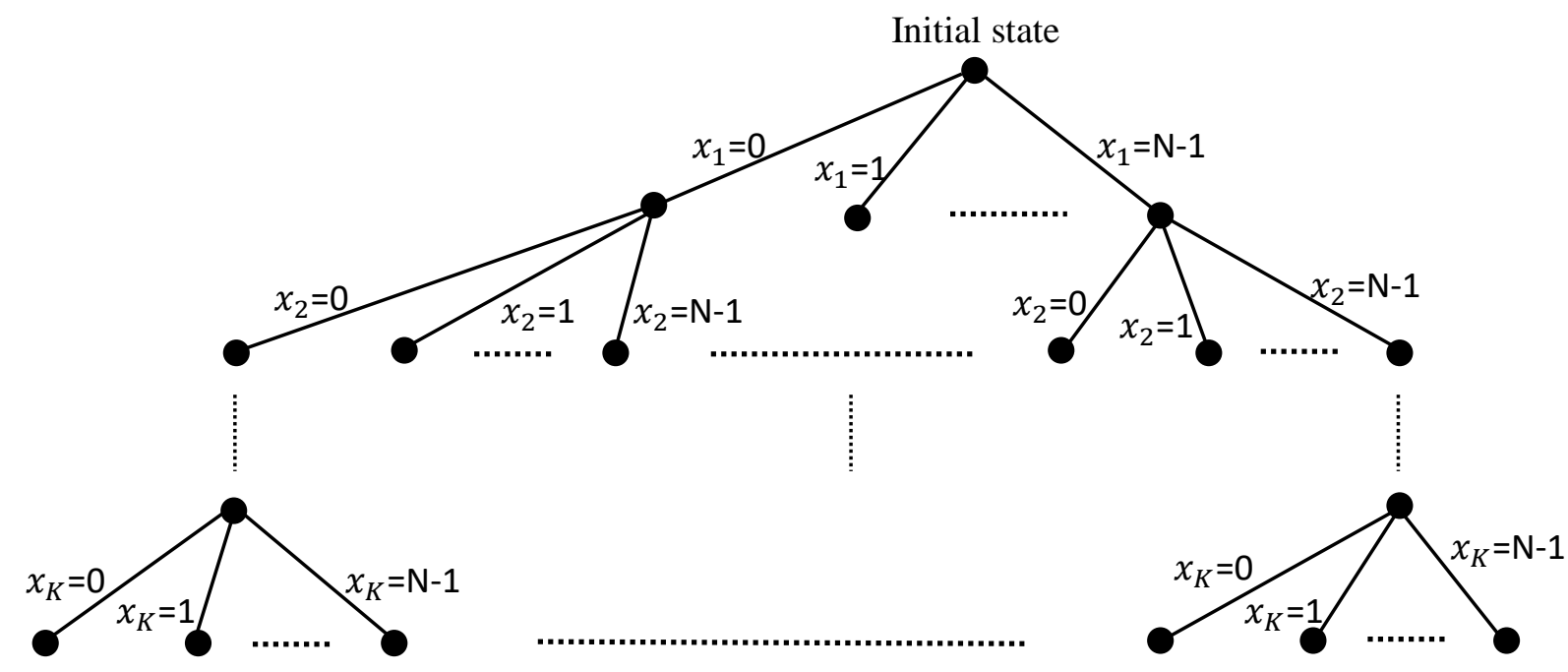

a)
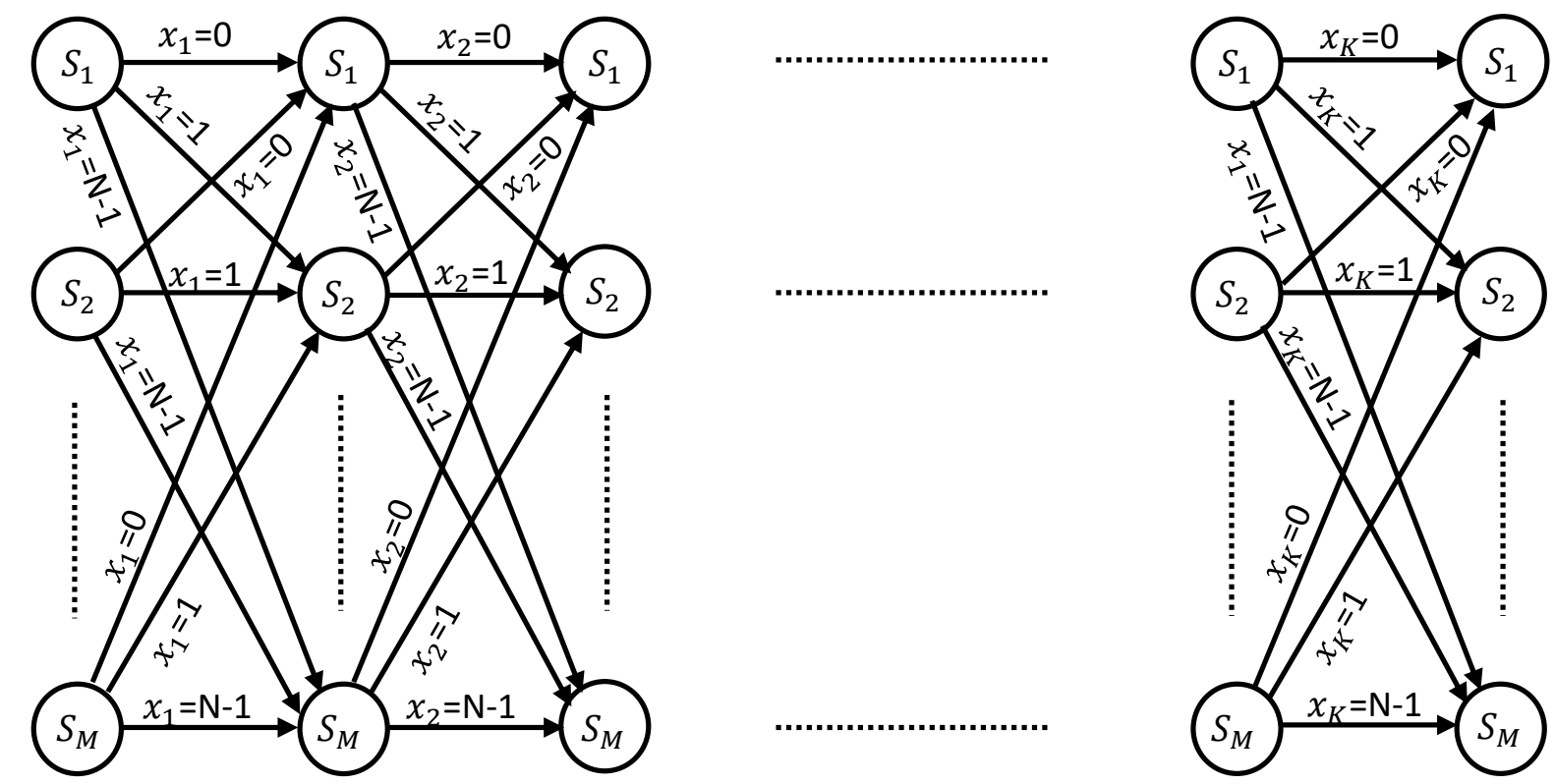

b)

Figure 3.8. Tree diagram of Viterbi algorithm (a) and trellis diagram of the Viterbi algorithm (b) 
Two generalized representations of the Viterbi algorithm are given in Figure 3.8. The first representation is called the tree diagram (Figure 3.8(a)). Its complexity and size increase exponentially with the length of the input sequence and thus is impractical for any but the shortest sequences. That is why the second representation (Figure 3.8(b)), known as a trellis diagram, is the most widespread representation of the Viterbi algorithm. In these representations the most important parameters of MLSE are introduced. The data at the input of the trellis is denoted by $x_{k}$, where $k$ is an integer number between 1 and $K$. The parameter $K$ represents the number of transitions or the number of stages of the trellis. The larger this number the more accurate the MLSE equalizer but also the higher the complexity. Another important parameter is the so-called MLSE memory length, denoted by $L$. In order to provide the best results this value should be equal to the channel memory (i.e. the number of symbols affected by ISI, see Figure 3.9). The memory length $L$ should not be confused with $K$, the length of the trellis. The two should however be directly proportional in order to achieve optimum performance and $K$ should always be larger than the MLSE memory length. The detected symbols at the beginning of the trellis are not very reliable. That is why when using MLSE the trellises are overlapped. The length of the overlapping interval is denoted by $q$ and should be at least five times the memory length [19]. Increasing the overlapping interval beyond $5 \mathrm{~L}$ will provide very small gains which do not justify the increase in complexity.

$$
q \geq 5 L
$$

Each stage of the trellis consists of a constant number of states, $M$. The number of states is determined by two other parameters: $N$, the number of levels of the modulation format (e.g. for PAM-4 the number of levels $N=4$ ) and $L$, the memory length.

$$
M=N^{L}
$$

In relation to (49) the number of branches between two stages of the trellis, $N B$, can also be defined:

$$
N B=N^{L+1}
$$

Let us now discuss how the Viterbi algorithm actually works. As previously stated in this chapter, the MLSE is the optimum detector for channels with ISI and additive white Gaussian noise (AWGN). Let's assume a channel with a memory length of $L$, as the one shown below. 


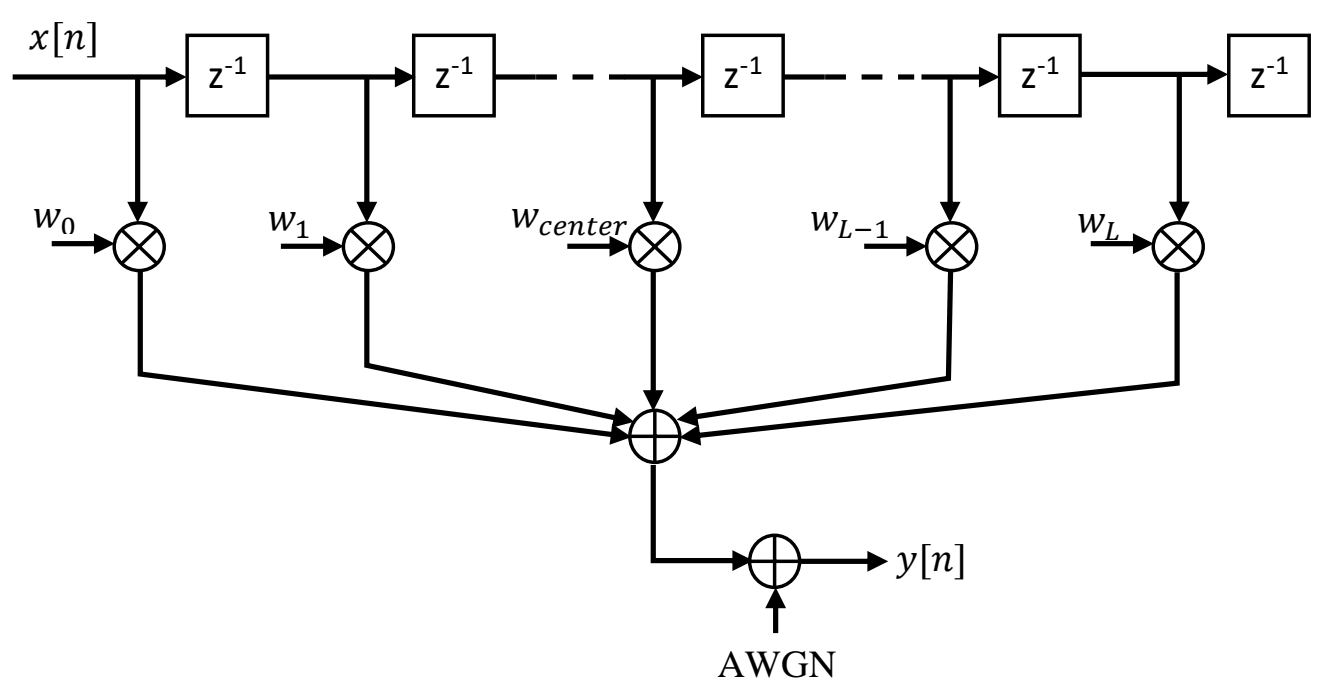

Figure 3.9. Block diagram of a channel affected by ISI and AWGN

The memory length of the MLSE is also denoted by $L$ and should be equal to the channel memory. The input data to the MLSE equalizer is denoted as $x$ and the transmitted modulation is PAM- $N$, $N$ representing the number of levels. The Viterbi trellis will have $M$ states (see equation (49)) and $N B$ branches (see equation (50)). For each transition from one state to another (i.e. for each received symbol) the branch metrics are calculated. This is done by calculating the Euclidian distance between the current received sample and the expected value for each branch. At each stage in the trellis there will be $N$ branches converging in each state. The new stage state metrics are calculated by summing each of the $N$ branches with the metric of the previous states corresponding to each branch and keeping the lowest of these values. This process is repeated for each stage until the end of the trellis is reached. Once the metrics of all states and branches in the trellis have been calculated a trace-back process is started in order to detect the most probable sequence. The first step of this process is to find the state in the last stage of the trellis with the lowest metric. From this state the trellis is processed backwards, at each stage going from the current state to the state with the lowest metric. Each chosen transition from one state to another will give us one detected symbol.

In order to better illustrate the Viterbi algorithm let's consider a numerical example. For simplicity let's assume we transmit a PAM-2 (or OOK) signal over a $10 \mathrm{~km}$ link at a speed of $56 \mathrm{Gbps}$ and that we use a MLSE equalizer with a memory length $L=2$ to detect the data. That means that we have a 4-state MLSE and eight branches for each transition and for each of these branches a metric based on a predefined channel model (see Metrics table in Figure 3.10). The scenario described above is implemented in simulation. The MLSE is stopped after processing the first 100 symbols so we can have a look at how the Viterbi algorithm operates. The trellis for symbols 101 to 108 together with all the relevant numerical values are displayed below. 


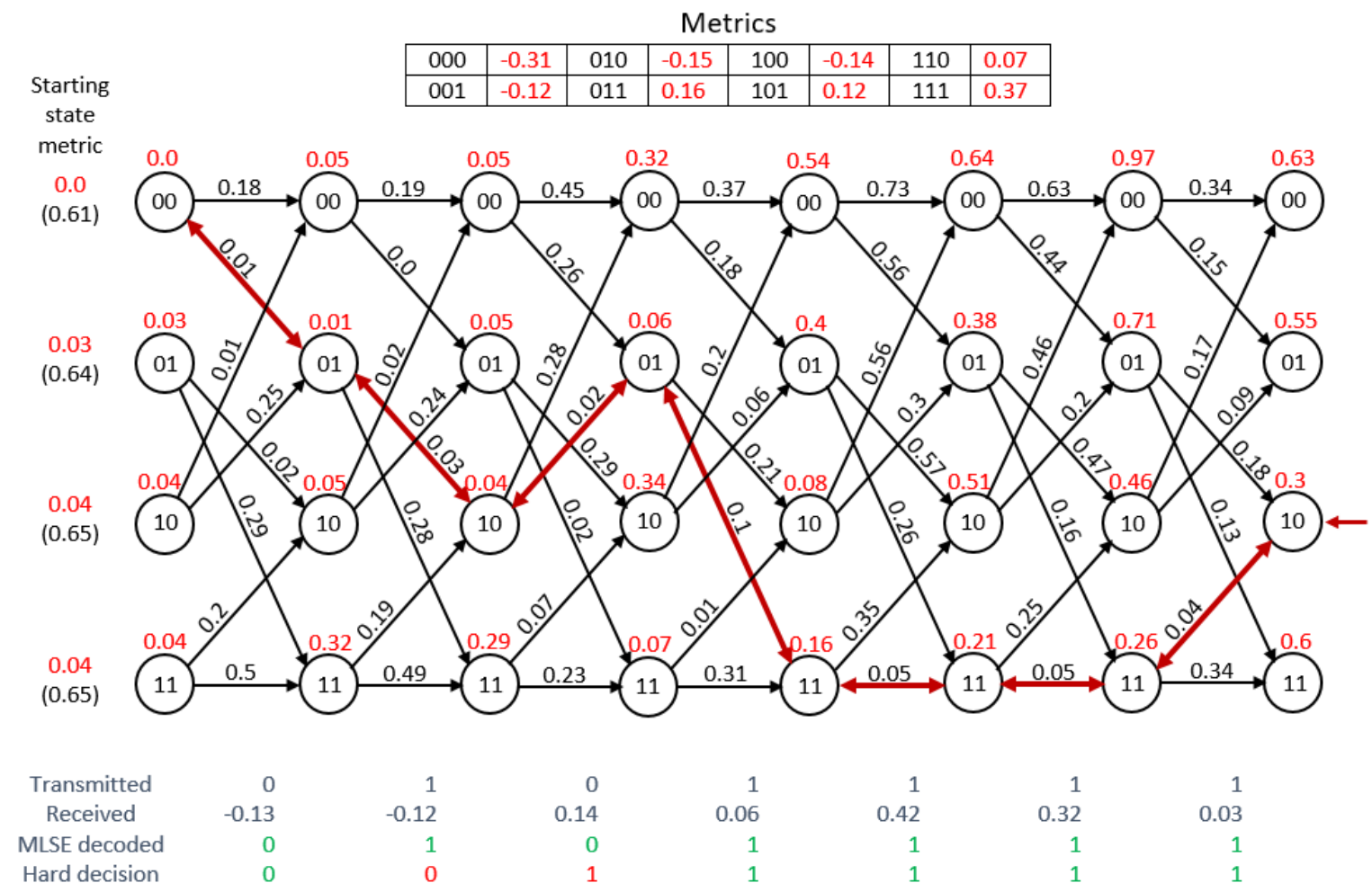

Figure 3.10. Numeric example of the Viterbi algorithm (trellis diagram decoding)

In the trellis diagram the 'shortest' trace-back path is highlighted with red and below it the transmitted symbols, received values, the MLSE detected symbols and the hard decision detection symbols are shown. It can be seen that due to the high $C D$ values the hard decision (the optimum threshold used in this example is -0.04 , lower means 0 , higher means 1 ) detection fails for two of the symbols in Figure 3.10. On the other hand, the MLSE equalizer is able to properly detect all the transmitted data. Please note that for this particular scenario the trellis length should be at least 10. However, due to space limitations and in order to keep the diagram readable, the length of the diagram from Figure 3.10 was cut down to eight stages.

In order for the MLSE to work the metrics for each possible state transition have to be calculated. Just like in the case of the taps of the FFE, the state transition metrics for the MLSE can be obtained in two ways: training mode and blind mode. In training mode a known symbol sequence is transmitted. At the receiver side, for each of the state transitions the corresponding received values are averaged in order to calculate the metric. If we consider the example in Figure 3.10 the training would start by taking the first state transition in the table, '000', look for this pattern in the transmitted data and for each time the '000' sequence is detected its corresponding received value is saved. After all the training sequence has been scanned the metric for ' 000 ' is calculated by doing the mean of all the saved values. Then the process is repeated for ' 001 ' and so on until the metrics for all state transitions are computed. 
In blind mode the process is the same only now the transmitted sequence is unknown and thus hard decision detection is required first. Of course that means that sometimes the detection is going to be wrong, thus the metrics calculated in blind mode might not be as accurate as the ones from training mode and longer sequences might be needed to acquire good metrics. The blind training can be performed also adaptively, meaning that the state transition metrics are updated constantly as new data is received. In real life implementations of MLSE for IM-DD optical transmissions an adaptive training is not always required since the optical channel is relatively stable. Usually for each transmission scenarios which the MLSE should cover a channel model (i.e. the values of state transition metrics) is trained offline.

The classic MLSE normally operates at one sample per symbol. However, in situations where the system performance after the MLSE equalizer is not good enough, there is the option of using two or more samples per symbol. This type of MLSE algorithm is referred to as fractionally spaced MLSE [61]. In this case for each state transition a number of metrics equal to the number of samples per symbol are calculated. In general, fractionally spaced MLSE depends less on timing recovery and sampling phase and provides better performance than the MLSE relying on only one sample per symbol, at the cost of increased complexity.

\subsubsection{Simulation and experimental analysis of MLSE performance}

For 100G non-coherent solutions [62] MLSE can be an attractive alternative for dealing with bandwidth limitations and CD. These solutions usually employ OOK as the modulation format

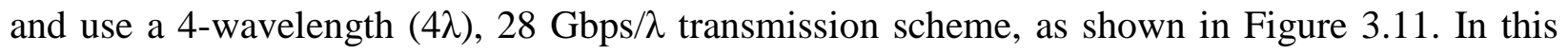
section the MLSE is shown to enable transmissions over $80 \mathrm{~km}$ of SSMF (in simulation and experimentally) employing transmission schemes as the one presented above and using low-cost $10 \mathrm{G}$ bandwidth components.

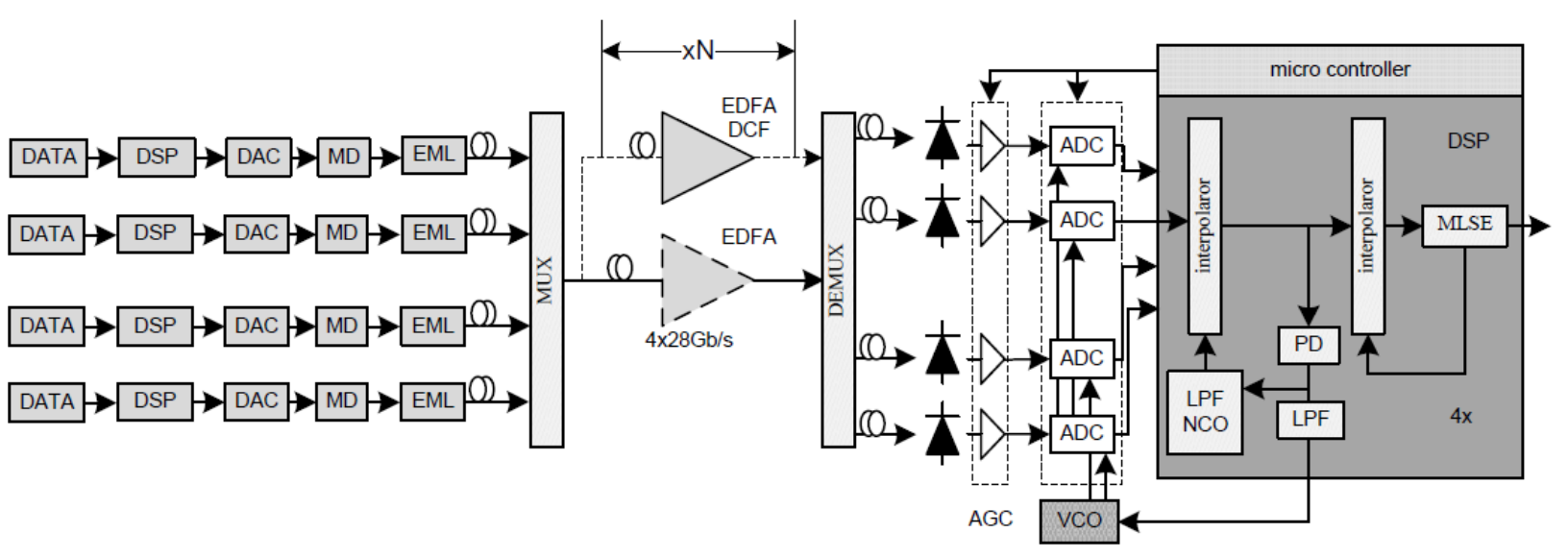

Figure 3.11. Block diagram of 100G WDM optical transmission system

Implementations of multi-lambda optical transmission systems, like the one in Figure 3.11, are too complex and required too many components to be efficiently implemented experimentally. When designing such a solution the first step is to prove the feasibility of a single-lambda version. For 
example, if a single-lambda transmission of OOK at 28 GBaud (25 GBaud for data, the rest for FEC) is proven at BERs below a certain threshold (which is dependent on the FEC being used) then the proposed solution might be feasible for the complete $100 \mathrm{G}$ product.

A simulation of a single-wavelength version of the system in Figure 3.11 was implemented. In order to evaluate MLSE performance with one and two samples per symbol for an OOK transmission, a 16-state MLSE is simulated, the CD is set to $700 \mathrm{ps} / \mathrm{nm}$ and the OSNR to $19 \mathrm{~dB}$. Transmitter and receiver bandwidths were set to 10 and $20 \mathrm{GHz}$. BER performances over two symbols are presented in Figure 3.12(a). In the case of $20 \mathrm{GHz}$ bandwidth, at the best sampling point, two samples per symbol achieved about six times better performance than single sample MLSE. Two samples per symbol MLSE is also sensitive to sampling phase because 16-state MLSE is at the limit for the simulated CD value. The bandwidth decreasing by a factor of two leads to much worse performance of a two-sample MLSE while single-sample MLSE did not suffer significantly. The single-sample MLSE performs better at the best sampling point.

It is interesting to see what is the maximum achievable transmission distance when employing a 16-state MLSE equalizer. For this purpose another simulation is performed where the transmission distance is varied from 0 to $100 \mathrm{~km}$ (with the distortion value set to $17 \mathrm{ps} / \mathrm{nm} / \mathrm{km}$ ) and the OSNR is varied from 10 up to $40 \mathrm{~dB}$. The bandwidth of the transmitter and receiver are set to $25 \mathrm{GHz}$ and the data rate is 28 GBaud. The goal is to determine the lowest OSNR values where the BER is below $10^{-3}$. The performance of an OOK transmission without MLSE is compared with that of an OOK transmission which employs a 16-state MLSE (with one or two samples/symbol). The results of this simulation are displayed in Figure 3.12(b). The OOK without equalization can reach up to $16 \mathrm{~km}$. Adding the 16-state MLSE increases the transmission length by more than four times, where the single-sample version of MLSE enables links of up to $80 \mathrm{~km}$ and the two-sample MLSE extends the reach to $88 \mathrm{~km}$ and improves also the OSNR requirements.
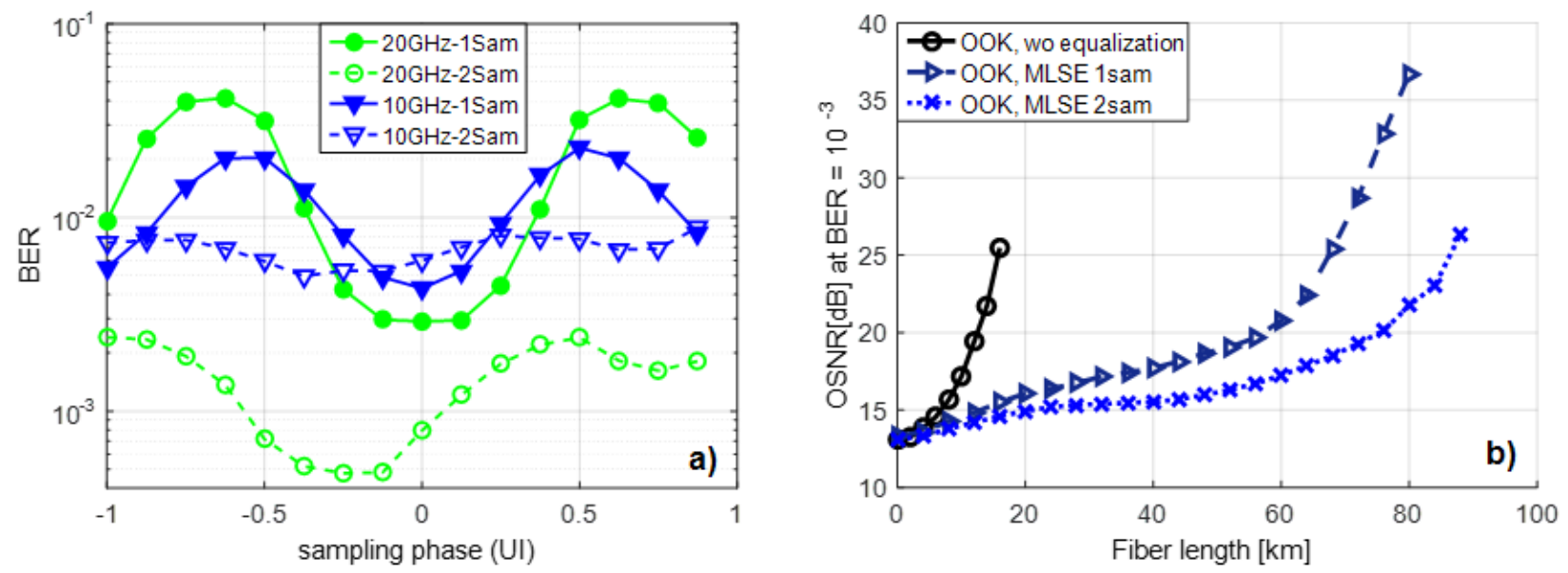

Figure 3.12. Simulation comparison between one Sample/Symbol and two Sample/Symbols 16-state MLSE for a $28 \mathrm{GBaud}$, OOK, CD = $700 \mathrm{ps} / \mathrm{nm}$ transmission (a) and simulation of achievable transmission length versus required OSNR for a $28 \mathrm{GBaud,OOK}$ system, with and without a 16-state MLSE 
The next step was to use experimental data to check the simulation results. We used the experimental setup presented in Figure 3.13. At the transmitter's side, a PRBS is used to generate the offline data using MATLAB. Pre-distortion of the signal is performed to compensate for the limited bandwidth of the transmitter. Then, the pre-distorted data are up-sampled to two Sam/s and are loaded to a DAC with an 8-bit resolution and $13 \mathrm{GHz} 3-\mathrm{dB}$ analog bandwidth. The DAC operates at $56 \mathrm{GSam} / \mathrm{s}$ leading to the generation of $28 \mathrm{Gbps}$ NRZ-OOK electrical signal at its differential outputs which are fed into a 25-GHz 3-dB bandwidth RF driver and then sent to a 10GHz transmitter optical sub-assembly (TOSA). The TOSA consists of a distributed feedback laser (DFB) and EAM modulator integrated in the same module. Next, the 28-Gb/s NRZ-OOK optical signal is launched over SSMF, amplified by an EDFA, and filtered through a 50-GHz optical filter. At the receiver side, the received optical power is changed using a variable optical attenuator (not presented in the figure) and the signal is finally detected on a 10-GHz ROSA. The ROSA consists of a $\mathrm{p}-\mathrm{i}-\mathrm{n}$ photodiode integrated together with a TIA. The received electrical eye diagrams in a BTB case and after $80 \mathrm{~km}$ for the received optical power of Pin=-10 dBm are also shown in Figure 3.13. The $80-\mathrm{km}$ eye diagram is completely closed, indicating that without enhanced equalization recovery is impossible.

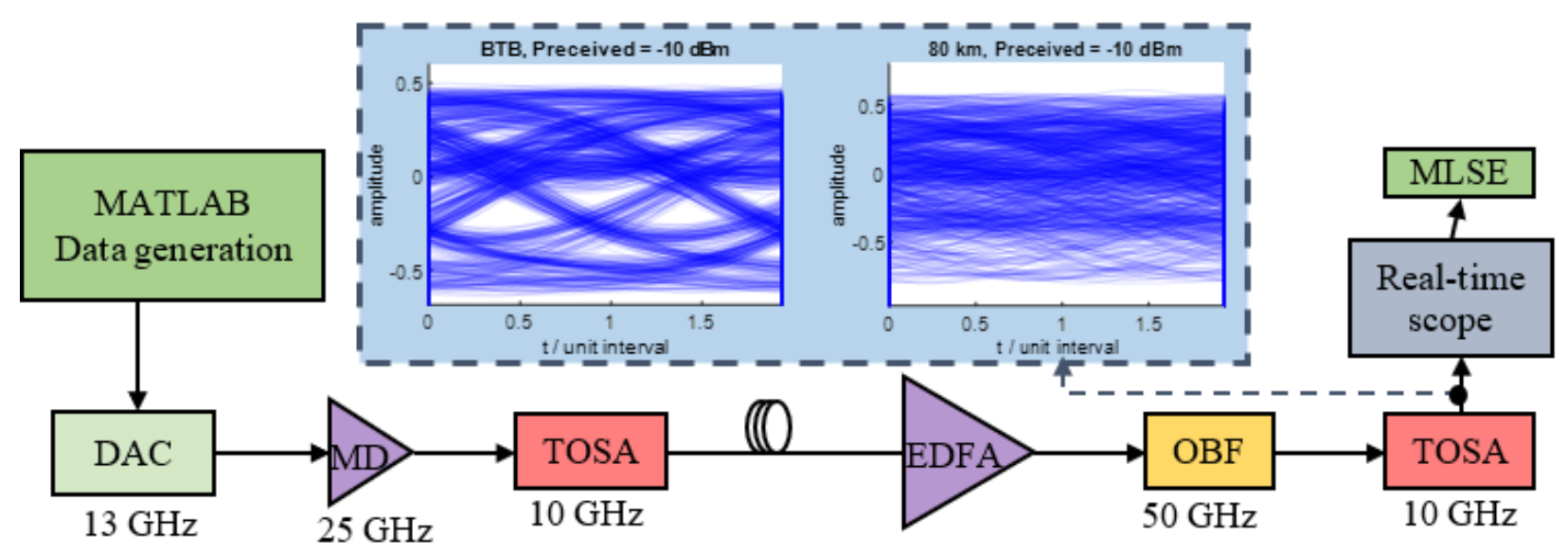

Figure 3.13. Experimental setup for testing one samples/symbol and two samples/symbol MLSE performance

The performance of 1-sample and 2-sample MLSE was estimated at BERs between $10^{-3}$ and $10^{-4}$. Links of 0,40 , and $80 \mathrm{~km}$ at input optical power of $-18,-16$, and $-14 \mathrm{dBm}$, respectively, were analyzed. A 16-state MLSE was used in the first two cases while a 64-state MLSE is simulated in the 80-km link. BER variations over two UIs are presented in Figure 3.14(a). One and two samples MLSE provide similar performance. In the BTB case and 40-km link, two samples are more resilient to the sampling phase while this advantage disappears at very high CD value after the 80 $\mathrm{km}$ link. Note that the single-sample MLSE equalizer has slightly better performance at the best sampling phase compared to the two-sample MLSE equalizer. In general, based on these results, it can be concluded that two samples per symbol are not required in strongly bandlimited systems. This agrees with the simulation results presented in Figure 3.12(a). One of the most critical points 
is to find the best sampling phase. Sampling phase variations introduce serious penalties in singlesample MLSE. We analyzed the BER minima and maxima closest to the sampling phase 0 . In the $80-\mathrm{km}$ case, at 0.5 UI phase, BER is greater than $10^{-2}$ while the minimum BER of $5 \times 10^{-5}$ is achieved at a -0.25 phase. For the same link, two samples yielded a BER minimum equal to $7 \times 10-5$ at a 0.25 while the maximum BER of 0.002 is located at a 0.375 phase. In this region, one sample experienced one-decade higher BER variations than a two-sample MLSE.
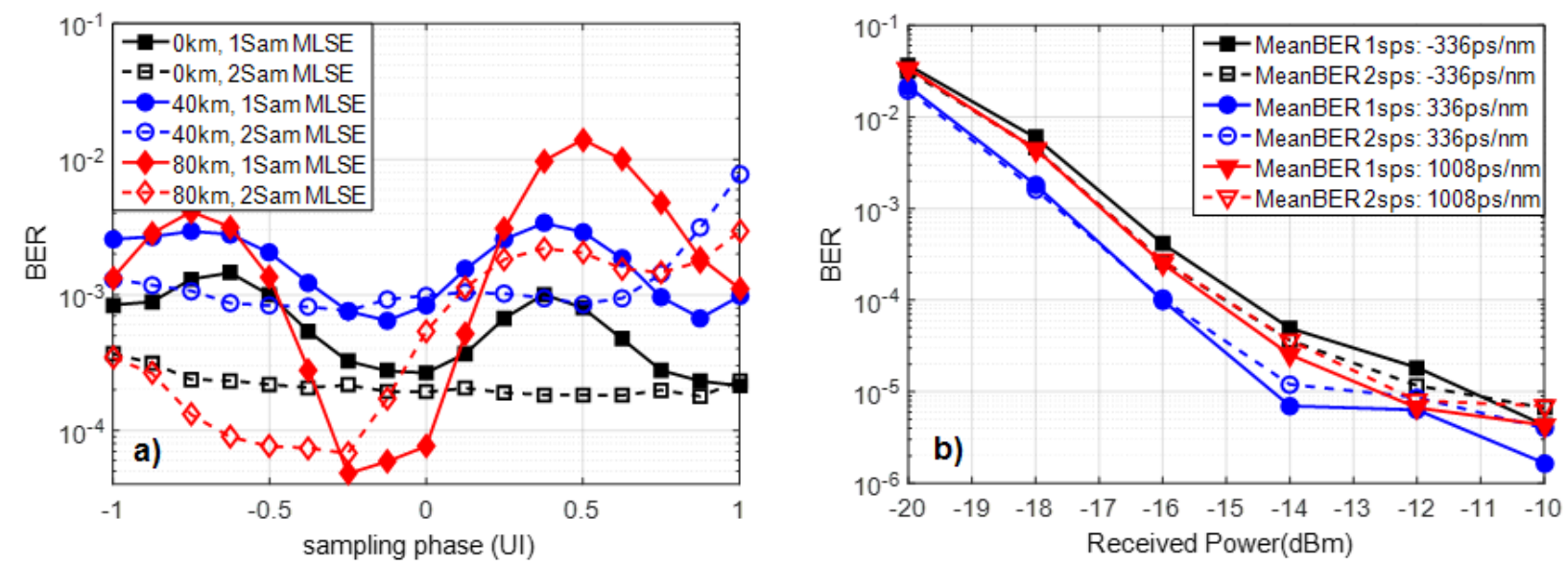

Figure 3.14. Experimental performance comparison between one sample/symbol and two sample/symbol MLSE for a 0, 40 and $80 \mathrm{~km}$ optical link (a) and received power sensitivity performance comparison (at 336, 336 and $1008 \mathrm{ps} / \mathrm{hm}$ ) between one sample/symbol and two sample/symbol MLSE (b)

In the next experiment, we used three links with CD values of $-336,336$, and $1008 \mathrm{ps} / \mathrm{nm}$. Negative dispersion was achieved by using a dispersion compensating fiber (DCF) fiber with negative dispersion. Optical power was varied from -10 to $-22 \mathrm{dBm}$. The 64-state MLSE equalizer was used in $1008 \mathrm{ps} / \mathrm{nm}$ link while in the other two cases, a 16-state MLSE is simulated. Minimum BER estimated at the best sampling phase versus input optical power for one- and two-sample MLSE is shown in Figure 3.14(b). BER is averaged over three data sets to get more accurate results. Two samples per symbol MLSE performs slightly better at negative dispersion. In the other two channels, there was no significant difference. This is an additional proof that a much more complex fractionally spaced MLSE is not required in the transmission scenario utilizing cheap optical components with cut-off frequency lower than the Nyquist frequency. 


\section{Chapter 4 DSP techniques for multilevel modulation formats}

In the last years the global internet traffic has seen a constant increase and estimates predict a continuation of this trend [2]. For the data center and short-reach metro-access sectors, where IMDD transmissions are preferred, the current $4 \lambda \times 10 \mathrm{G}$ and $4 \lambda \times 25 \mathrm{G}$ WDM implementations are trying to keep up with the ever-increasing demand. For this reason, an upgrade to 400G metroaccess WDM optical networks is currently in progress [6]. Since cost is a major concern in shortreach systems, it is preferable that future 400G WDM networks are implemented using inexpensive components. This type of components imposes heavy bandwidth restrictions which make the traditional OOK implementation impractical in a high-speed/low-bandwidth scenario. While single-wavelength OOK has been proven at speeds of $100 \mathrm{Gbps}$ [63], [64] or even higher [65], very high bandwidth components or complex multiplexing schemes are required for such implementations. This is why research is focused towards higher order modulation formats like PAM-4 [66], [67], DMT [11] or CAP [12]. Out of these modulation schemes PAM-4 seems to be the most likely candidate [68]. That is why the main focus of this chapter is on multilevel intensity modulation formats such as PAM-4 or PAM-8. By 'multilevel' we refer to any optical intensity modulation that has more than two amplitude levels (i.e. more amplitude levels than OOK).

\subsection{Multilevel intensity modulation generation}

Because cost and power consumption are major concerns when it comes to short-reach and datacenter interconnection systems, intensity modulation is the only viable solution at the moment. In recent years OOK has been the preferred solution for short-reach optical transceiver products. Its success is due to its simplicity. It is easy to generate, easy to detect at the receiver side and easy to design DSP for. However, in the last few years, the speed requirements have increased very fast, while the bandwidth of the commercially available components did not increase at the same rate. This has resulted in OOK not performing very well at high baud rates or requiring multiple DACs and/or multiplexers (MUX) [63], [65]. In order to avoid or at least mitigate these complications with OOK, the research focus in the optical telecommunications industry has shifted to multilevel PAM, especially PAM-4. 


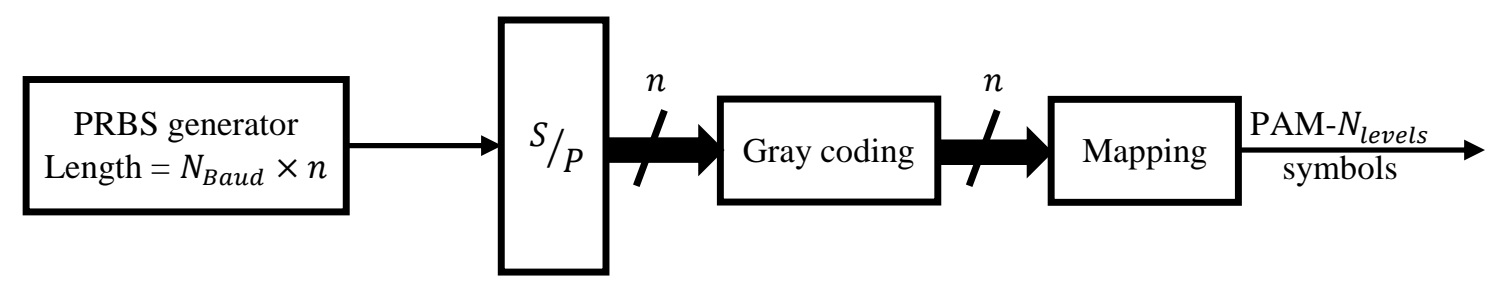

Figure 4.1. PAM-n generation

To generate any higher order PAM the block diagram in Figure 4.1 can be employed. The first step is to define the number of bits/symbol of the modulation $-n$, also referred to in this work as the order of the modulation. This number would be, for example, equal to 2 for PAM- 4 or 3 for PAM-8. The number of levels of a PAM modulation is equal to 2 to the power of $n$ :

$$
N_{\text {levels }}=2^{n}
$$

A PRBS is generated with a length equal to the number of symbols that we want to transmit multiplied by the number of bits/symbol.

$$
P R B S_{\text {length }}=N_{\text {baud }} * n
$$

It must be noted that in experiments the PRBS length cannot be too long. This length is limited by the memory of the DAC or AWG, which is typically $500 \mathrm{kB}$ to $1 \mathrm{MB}$. Thus, depending on the baud rate of the transmitted signal, the $N_{\text {baud }}$ must be equal or smaller than the memory of the DAC divided by the number of bits/sample of the DAC (i.e. usually 8). That is why in most experiments the length of the PRBS is limited to values around $2^{15}$ to $2^{16}$. In order to generate a continuous, time unlimited signal the DAC will repeat the data in its memory over and over again.

$$
\max \left(N_{\text {baud }}\right)=\frac{\text { Memory }_{D A C}}{N_{\text {sps }_{D A C}} * N_{\text {bits }_{\text {sample }}} \text { DAC }}
$$

Once the PRBS is generated, the bit stream is converted from serial to parallel, with the number of parallel streams equal to $n$, the order of the modulation. The next step is to perform Gray encoding on the parallel bit stream. This is done because levels which are neighbors can have more than one bit difference between them (e.g. level 2 and 3 of PAM-4 - 10 and 01 have a two bits difference), which means that a symbol error after transmission can result in two or more bit errors. The Gray coding remaps the bits corresponding to the modulation levels in such a way that any level will have only one bit difference when compared to its two nearest neighbors. If the most significant bit (MSB) is considered the first and left-most bit and the least significant bit (LSB) is considered the last and right-most bit, then the Gray coding can be written as:

$$
\text { Grey bits }=b_{1}, b_{1} \oplus b_{2}, \ldots, b_{n-1} \oplus b_{n}
$$

The effective Gray coding for PAM-4, PAM-8 and PAM-16 modulations is shown in Table 1. The last step of generating the PAM is to map the Gray encoded bits onto their respective PAM symbols. The mapping for PAM-4, PAM-8 and PAM-16 modulations is presented in Table 1. 
Table 1. Gray coding and mapping for OOK, PAM-4, PAM-8 and PAM-16

\begin{tabular}{|c|c|c|c|c|c|c|c|c|c|c|c|c|}
\hline Modulation & \multicolumn{4}{|c|}{ Serial-to-parallel } & \multicolumn{4}{|c|}{ Gray coding } & \multicolumn{4}{|c|}{ Mapping } \\
\hline OOK & \multicolumn{4}{|c|}{0} & \multicolumn{4}{|c|}{0} & \multicolumn{4}{|c|}{0} \\
\hline & \multicolumn{4}{|c|}{1} & \multicolumn{4}{|c|}{1} & \multicolumn{4}{|c|}{1} \\
\hline PAM-4 & \multirow{2}{*}{\multicolumn{4}{|c|}{00}} & \multicolumn{4}{|c|}{$00 \quad 11$} & \multicolumn{4}{|c|}{$0 \quad 2 / 3$} \\
\hline & & & & & \multicolumn{4}{|c|}{$01 \quad 10$} & \multicolumn{4}{|c|}{$1 / 3 \quad 1$} \\
\hline \multirow[t]{4}{*}{ PAM-8 } & \multicolumn{4}{|c|}{$\begin{array}{ll}000 & 100\end{array}$} & \multicolumn{4}{|c|}{$000 \quad 110$} & \multicolumn{4}{|c|}{$\begin{array}{ll}0 & 4 / 7\end{array}$} \\
\hline & \multicolumn{4}{|c|}{101} & \multicolumn{2}{|r|}{001} & \multicolumn{2}{|l|}{111} & \multicolumn{4}{|c|}{$1 / 7 \quad 5 / 7$} \\
\hline & \multicolumn{4}{|c|}{110} & \multicolumn{4}{|c|}{101} & \multicolumn{4}{|c|}{$2 / 7 \quad 6 / 7$} \\
\hline & \multicolumn{4}{|c|}{111} & \multicolumn{4}{|c|}{100} & & $3 / 7$ & 1 & \\
\hline \multirow[t]{4}{*}{ PAM-16 } & 0000 & 0100 & 1000 & 1100 & 0000 & 0110 & 1100 & 1010 & 0 & $4 / 15$ & $8 / 15$ & $12 / 15$ \\
\hline & 0001 & 0101 & 1001 & 1101 & 0001 & 0111 & 1101 & 1011 & $1 / 15$ & $5 / 15$ & $9 / 15$ & $13 / 15$ \\
\hline & 0010 & 0110 & 1010 & 1110 & 0011 & 0101 & 1111 & 1001 & $2 / 15$ & $6 / 15$ & $10 / 15$ & $14 / 15$ \\
\hline & 0011 & 0111 & 1011 & 1111 & 0010 & 0100 & 1110 & 1000 & $3 / 15$ & $7 / 15$ & $11 / 15$ & 1 \\
\hline
\end{tabular}

Depending on the desired baud rate and experimental scenario, after the PAM symbols are generated, some additional DSP might be employed (e.g. digital pre-emphasis, pulse shaping, resampling) before the data is ready for the DAC. How to prepare the data for the DAC has been explained already in chapter 2.1.1. Additional transmitter DSP techniques, like DPE are discussed later in this thesis in chapters 5.4 and 5.5.

\subsection{MLSE for multi-level PAM}

As stated before and as in the case of OOK, the most common DSP employed in higher order PAM optical systems are the FFE and the MLSE [15], [27]. The FFE is very useful when equalizing the channel and in dealing with bandwidth limitations. When moving from OOK to PAM-4 or PAM8 the FFE has the advantage that its complexity increases linearly with the order of the modulation. Its performance is evaluated in the context of several other DSP techniques and experiments and thus no further details are given here.

The general architecture and performance of MLSE were described in chapter 3.3 and thus are not discussed here again. In this chapter we analyze the performance of MLSE with one and two samples per symbol, when PAM-4 or PAM-8 are employed as modulation formats. The first step was to design a simulation which considers a channel with CD and ASE noise and a 64-state MLSE equalizer and test the performance of OOK, PAM-4 and PAM-8 in this scenario. Compared with OOK, PAM-4 requires higher OSNR values in order to achieve the same BER. However, at the same bit rate, PAM-4 requires half the bandwidth and has better CD tolerance, as can be seen in Figure 4.2, where no bandwidth limitations were imposed and 64 states MLSE was used for equalization. OOK allows us to reach around $80 \mathrm{~km}$ fiber length while maintaining the BER below $10^{-3}$. For the same BER threshold PAM-4 can be used for fiber lengths of up to $120 \mathrm{~km}$. Increasing the order of the modulation further to PAM-8 allows for even longer transmissions (for the scenario 
described earlier in this paragraph) of up to roughly $140 \mathrm{~km}$. Again, this comes at the cost of increased OSNR sensitivity.

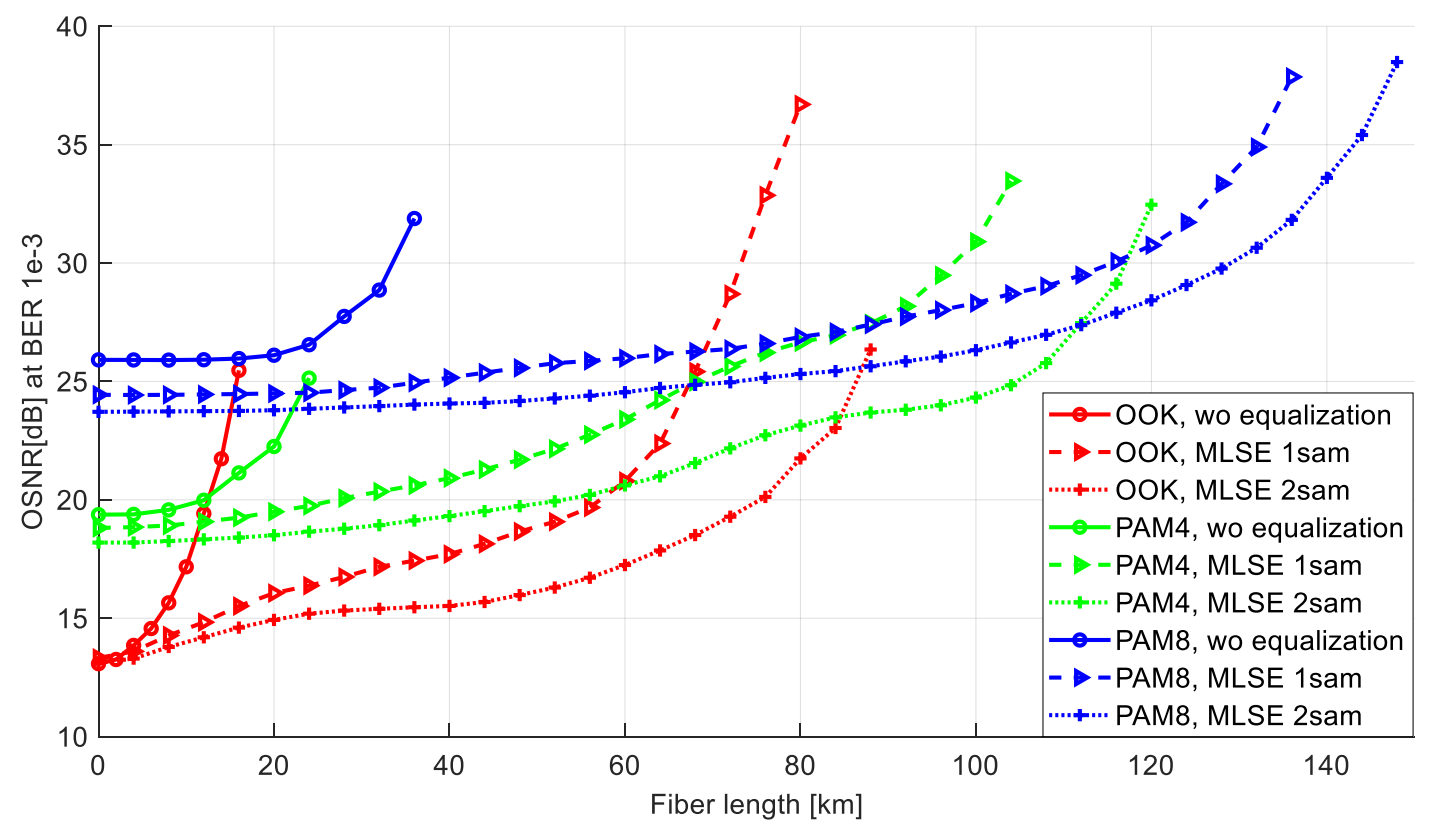

Figure 4.2. Transmission reach for 28 Gbps OOK (red), PAM-4 (green) and PAM-8 (blue) when using no DSP or a 64-state MLSE (1-sample or 2-sample) - simulation results

To assess the performance of one- and two-sample per symbol MLSE in the context of PAM-4 modulation, receiver and transmitter bandwidths of $10 \mathrm{GHz}$ and $20 \mathrm{GHz}$ are simulated, the OSNR is set to $24 \mathrm{~dB}$ and the $\mathrm{CD}$ to $170 \mathrm{ps} / \mathrm{nm}$. The baud rate is 14 GBaud, resulting in a bit rate of 28 Gbps. In Figure 4.4(a) are shown the simulation results with these parameters. As in the case of OOK modulation (see chapter 3.3.2), a 16-state MLSE was used. It must be mentioned that for PAM-4 the complexity of MLSE grows faster with the increase of memory bits (see equation (49)). In the case of $20 \mathrm{GHz}$ bandwidth the fractionally spaced MLSE performs two times better than its single sample counterpart, achieving a minimum BER of $4 \times 10^{-4}$, while the single sample MLSE achieves a minimum BER of $9 \times 10^{-4}$. When limiting the bandwidth to $10 \mathrm{GHz}$ the performance stays the same and even improves somewhat for the one sample per symbol MLSE. This can be attributed to the fact that a lower bandwidth also limits some of the noise while the MLSE can compensate almost entirely for the lost signal bandwidth.

As in the case of OOK, also for PAM-4 we setup an experiment to test the performance of fractionally spaced MLSE. The block diagram of this setup can be seen in Figure 4.3. Two PRBSs are generated and loaded into the BPG. The BPG then outputs two OOK signals which are fed to a couple of $25 \mathrm{GHz}$ bandwidth drivers. Next the amplified signals are sent to a $20 \mathrm{GHz}$ bandwidth IQ MZM which will modulate a CW laser and generate at its output the optical PAM-4 signal. The optical signal is then launched over SSMF of lengths 0,4 and $8 \mathrm{~km}$. The optical signal is amplified with the help of an EDFA and filtered by a $50 \mathrm{GHz}$ bandwidth optical bandpass filter (OBF). The optical eye diagrams after EDFA for 0 and $8 \mathrm{~km}$ can be seen in Figure 4.3. It shows that for $8 \mathrm{~km}$ 
the signal is already heavily distorted, more than can be attributed to $\mathrm{CD}$ alone. This is due to the chirp introduced by the IQ MZM when generating PAM-4 signal [69]. The receiver consists of a p-i-n photodiode. After the receiver a real-time oscilloscope is used to capture the data at a sampling rate of $50 \mathrm{GSamples/second.}$

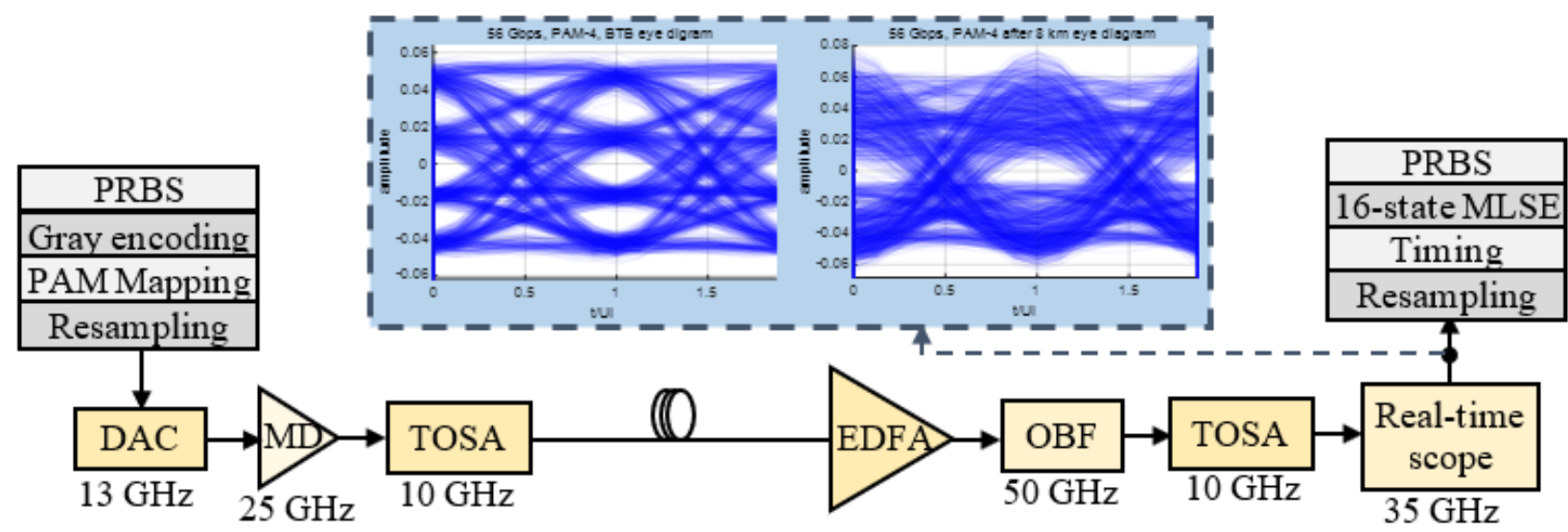

Figure 4.3. Experimental setup for testing MLSE performance in a 56 Gbps PAM-4 optical transmission
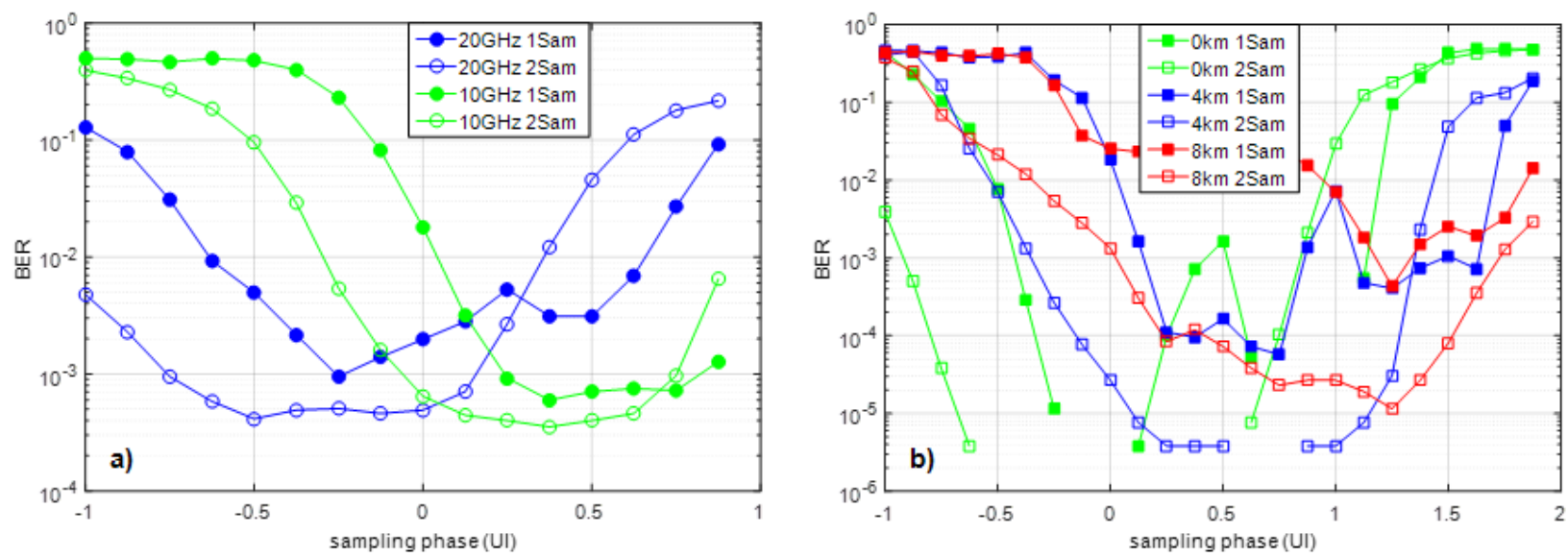

Figure 4.4. Simulation analysis on the performance of 16-state MLSE with CD of $170 \mathrm{ps} / \mathrm{nm}$ and 28 Gbps, PAM-4 (a) and BER variations in 56 Gbps, PAM-4 experiments after 0, 4, and $8 \mathrm{~km}$ links (b)

The performance of one- and two- sample MLSE equalizers for links of 0, 4 and $8 \mathrm{~km}$ is evaluated in Figure 4.4(b). For lengths of 0 and $4 \mathrm{~km}$ a 16-state MLSE was employed, while for $8 \mathrm{~km}$ a 64state MLSE was needed. The achieved BER for this experimental setup after MLSE equalization can be seen in Figure 4.4(b). The missing points in this figure represent a BER of 0. However, due to the limited amount of offline data that were processed ( $2^{19}$ symbols for each BER point), values below $10^{-5}$ are not very precise and a BER of 0 should be interpreted as a BER below $10^{-5}$. In the BTB case both the single sample and the fractionally spaced MLSE are able to eliminate all errors, the latter option being more stable. For the 4 and $8 \mathrm{~km}$ transmissions two samples perform much better. While with one sample MLSE we achieved BER of $5 \times 10^{-5}$ and $4 \times 10^{-4}$ for the 4 and $8 \mathrm{~km}$ links respectively, the 2 -sample MLSE will correct all errors when transmitting over a $4 \mathrm{~km}$ link and lower the BER to $10^{-5}$ for $8 \mathrm{~km}$. For both cases there is a significant improvement of over a 
decade (more than 10 times) in BER. It must be noted that the $3 \mathrm{~dB}$ bandwidth of the whole system in the PAM-4 experiment is around $20 \mathrm{GHz}$, such that the bandwidth limitations are less severe than in the case of the OOK experiment. Simulation results from Fig. 8 suggest that when bandwidth is not limited the MLSE equalizer with two samples will perform better.

In this section the capabilities of regular MLSE and of fractionally spaced MLSE equalizers have been demonstrated, both in simulations and experiments for multi-level PAM modulation techniques. Optical and electrical components for 10-G IM-DD systems can be used to carry a 28$\mathrm{Gb} / \mathrm{s}$ signal that is equalized by using the MLSE technique. For this purpose single sample MLSE gives approximately the same results as the fractionally spaced MLSE. Compared with two samples, a single sample MLSE decreases the application specific integrated circuit (ASIC) complexity by up to $30 \%$, depending on the level of parallelization and MLSE parameters selection, among others. If better components are used, such that the bandwidth is not too limited, then two samples MLSE equalization will bring significant gain in terms of BER which can overcome the cost of the $30 \%$ increase in complexity. Since in high baud rate IM-DD optical system the bandwidth of the components is rarely high enough (resulting in severe bandwidth limitations), the 1-sample MLSE is preferred for its lower complexity. Throughout the rest of this work the single sample MLSE is used, unless specified otherwise.

\subsection{Geometrical constellation shaping}

In this chapter the geometrical constellation shaping is introduced and its benefits are presented. A standard multi-level PAM signal at the transmitter side (before modulator) has equidistant levels. If we assume a minimum amplitude of $0 \mathrm{~V}$ and a maximum amplitude of $1 \mathrm{~V}$ then levels of OOK, PAM-4, PAM-8 and PAM-16 in the electrical domain are defined as in Table 1 (fourth column - 'mapping'). The eye diagrams of classic PAM-4, PAM-8 and PAM-16 before being loaded into the DAC are shown in Figure 4.5, where the equidistant levels can be clearly seen.
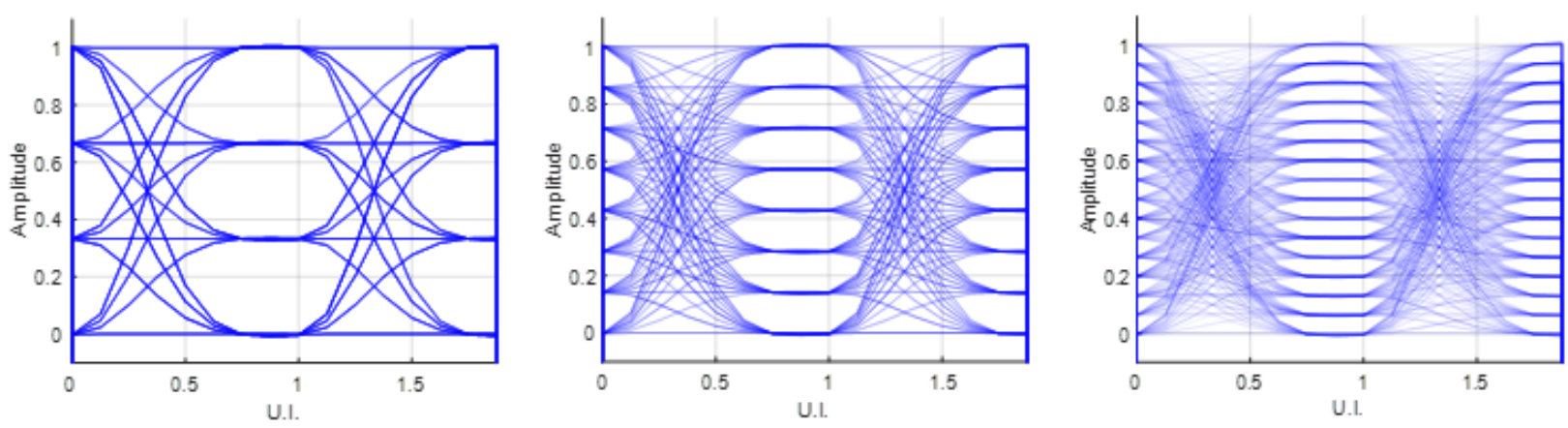

Figure 4.5. Equidistant levels PAM eye diagrams before DAC for PAM-4 (left), PAM-8 (middle) and PAM-16 (right)

In the case of intensity modulation formats the geometrical shaping of their constellations refers to the redistribution of the amplitude levels in such a way that they are no longer equidistantly distributed. This technique can be employed to mitigate the effects of white Gaussian optical noise 
or, as shown in section 4.3.2, to reduce the contribution of dispersion to the degradation of an optical transmission.

\subsubsection{Geometrical shaping for mitigating the effects of noise}

In an IM-DD optical transmission system there are two main types of noise - electrical and optical. The electrical noise is generated by the electrical components, such as the MD amplifier at the transmitter side and the PD and TIA at the receiver side. The optical noise is produced mainly by the EDFAs. For short-reach transmissions where EDFAs are not required (typically less than 20$30 \mathrm{~km}$ ) the optical noise can be ignored. On the other hand, when one or more EDFAs are present in the link the ASE noise becomes an issue. To explore further the effects of noise on the transmitted signal let us consider the following notations (see Figure 4.6): $x$ - the PAM signal at the input of the DAC, $u$ - the signal after the modulator, $z$ - the signal after EDFA and PD, $y-$ the signal after the ADC, $n_{e l, T x}$ - the electrical noise generated at the transmitter side, $n_{o p t}-$ the optical ASE noise generated by the EDFA and $n_{e l, R x}-$ the electrical noise generated at the receiver side. For simplicity the noise is assumed to be AWGN. While this is not always the case, this approximation is quite close to reality.

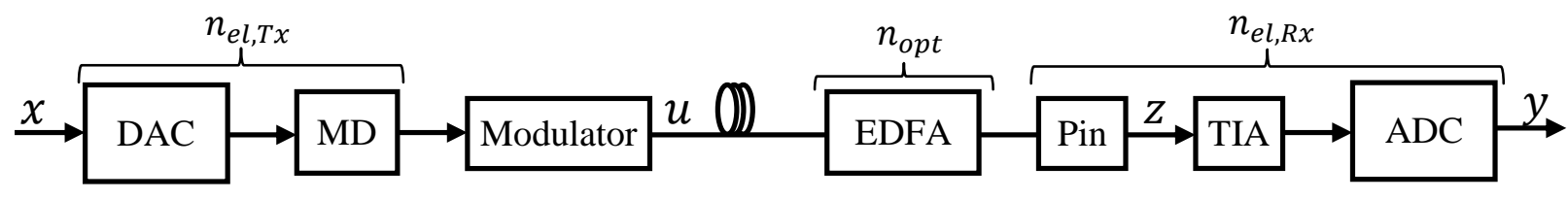

Figure 4.6. Transmission block diagram and signal notations

The signal $x$ is a PAM signal generated in MATLAB. The goal of this analysis is to determine the optimum amplitude levels of $x$ such that the influence of the noise and the BER of $y$ are minimized. The influence of each of the three noises defined above is minimized when the PAM signal onto which they are added has equidistant levels. Thus, if $x, u$ or $z$ have equidistant levels, the effect of $n_{e l, T x}, n_{\text {opt }}$ or $n_{e l, R x}$ respectively is at its lowest, as illustrated in Figure 4.7. So, if $x$ has equally spaced levels then the influence of $n_{e l, T x}$ on the BER would be minimized, if $u$ has equally spaced levels then the influence of the optical noise on the BER would be at its lowest and if $z$ has equally spaced levels then the influence of $n_{e l, R x}$ on the BER would be minimized.

Because of the modulator and Pin PD it is not possible to have all the three signals $(x, u$ and $z)$ with equidistant levels. Let's assume that the initial PAM signal generated in MATLAB has equally spaced levels (to which we will refer from now on as ESL) and that an EML is used as modulator. The signal $u$ at the output of the EML can be approximated with the square root of the transmitted signal:

$$
u=\sqrt{x+n_{e l, T x}}=\left(x+n_{e l, T x}\right)^{1 / 2}
$$


A PD receiver detects the power of the input optical signal, thus the signal after it can be expressed as:

$$
z=\left(u+n_{\text {opt }}\right)^{2}
$$

If we were to ignore the noise part of equations (55) and (56) then it would result that the levels of $x$ and those of $z$ are equal to each other and that they are ESL. Thus, it can be concluded that when an ESL PAM signal is generated at the transmitter side then the influence of the electrical noise on the performance of the system is minimized.
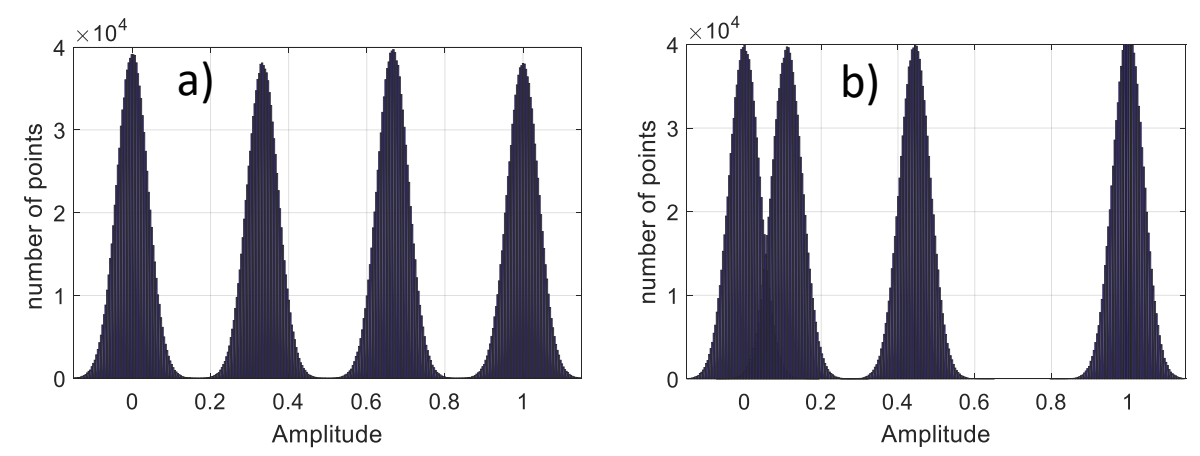

Figure 4.7. Influence of AWGN on ESL PAM-4 (a) and on unequally spaced levels PAM-4 (b)

If optical noise in the link is dominant over the electrical noise then we might wish to minimize its effects, meaning that the signal $u$ must have ESL. If the electrical noise at the transmitter side is ignored then:

$$
x=u^{2}
$$

Since $u$ is assumed to have ESL, then, based on equation (57), the levels of $x$ which minimize the effects of the optical noise are as defined in Table 2 for PAM-4, PAM-8 and PAM-16 modulations.

\begin{tabular}{|c|c|c|c|c|c|c|c|c|}
\hline Modulation & \multicolumn{4}{|c|}{$x$} & \multicolumn{4}{|c|}{$u=\sqrt{x}$} \\
\hline \multirow[t]{2}{*}{ PAM-4 } & \multirow{2}{*}{\multicolumn{4}{|c|}{$\begin{array}{lr}0 & 4 / 9 \\
1 / 9 & 1\end{array}$}} & \multicolumn{4}{|c|}{$\begin{array}{ll}0 & 2 / 3\end{array}$} \\
\hline & & & & & & $1 / 3$ & 1 & \\
\hline \multirow[t]{4}{*}{ PAM-8 } & \multicolumn{3}{|r|}{$16 / 49$} & & \multicolumn{2}{|r|}{0} & & \\
\hline & & $1 / 49$ & $25 / 49$ & & & $1 / 7$ & $5 / 7$ & \\
\hline & & $4 / 49$ & $36 / 49$ & & & $2 / 7$ & $6 / 7$ & \\
\hline & & $9 / 49$ & 1 & & & $3 / 7$ & 1 & \\
\hline PAM-16 & 0 & $16 / 225$ & $64 / 225$ & $144 / 225$ & 0 & $4 / 15$ & $8 / 15$ & $12 / 15$ \\
\hline & $1 / 225$ & $25 / 225$ & $81 / 225$ & $169 / 225$ & $1 / 15$ & $5 / 15$ & $9 / 15$ & $13 / 15$ \\
\hline & $4 / 225$ & $36 / 225$ & $100 / 225$ & $196 / 225$ & $2 / 15$ & $6 / 15$ & $10 / 15$ & $14 / 15$ \\
\hline & $9 / 225$ & $49 / 225$ & $121 / 225$ & 1 & $3 / 15$ & $7 / 15$ & $11 / 15$ & 1 \\
\hline
\end{tabular}

Table 2. Optimum modulation levels for minimizing the effects of optical noise

While when the electrical or optical noise are considered on their own the optimum amplitude levels of $x$ are easily determined, the optimization problem becomes more complex when both are 
present in the system at the same time. To solve this problem let's first expand on equations (55) and (56):

$$
\begin{aligned}
z=\left(u+n_{o p t}\right)^{2} & =\left[\left(x+n_{e l, T x}\right)^{\frac{1}{2}}+n_{o p t}\right]^{2} \\
& =x+n_{e l, T x}+2 \sqrt{x+n_{e l, T x}} * n_{o p t}+n_{o p t}^{2}
\end{aligned}
$$

Given the equation above, $y$ can be expressed as:

$$
y=z+n_{e l, R x}=x+n_{e l, T x}+2 \sqrt{x+n_{e l, T x}} * n_{o p t}+n_{o p t}^{2}+n_{e l, R x}
$$

Higher order noise elements like $n_{o p t}^{2}$ do not contribute significantly to the total noise and can be ignored when the standard deviation of $n_{\text {opt }}$ is much smaller than the amplitude difference between two neighboring modulation levels (which is generally the case). When considering AWGN the noise has a Gaussian distribution with the mean $\mu=0$ and a standard deviation $\sigma$. The effects of the noise on the amplitude of the signal in this case can be perfectly described by its standard deviation. Taking into account the previous considerations equation (59) can be rewritten as follows:

$$
y=x+\sigma_{e l, R x}+\sigma_{e l, T x}+2 \sqrt{x+n_{e l, T x}} * \sigma_{o p t}
$$

The electrical noise at the transmitter and receiver apply linearly onto the transmitted signal $x$, as shown by the first three elements on the right side of equation (60). Thus $\sigma_{e l, R x}$ and $\sigma_{e l, T x}$ are minimized when the levels of $x$ are equidistant. The optical noise however is added onto the square root of $x$ so, as shown also in Table 2, $\sigma_{o p t}$ is minimized for a squared distribution of the modulation levels. In order to optimize the levels of $x$ for all types of noise a level distribution in between the electrical optimum and the optical optimum is chosen, proportional to the standard deviations. Let's define first the total standard deviation of the noise as:

$$
\sigma_{\text {total }}=\sigma_{e l, T x}+\sigma_{o p t}+\sigma_{e l, R x}
$$

If we denote the linearly distributed levels (i.e. ESL - $[0,0.33,0.66,1])$ of the modulation as $x_{l, e q}$, then the optimum levels can be expressed as:

$$
x_{l_{\text {optimized }}}=\frac{\sigma_{e l, T x}+\sigma_{e l, R x}}{\sigma_{\text {total }}} * x_{l, e q}+\frac{\sigma_{o p t}}{\sigma_{\text {total }}} * x_{l, e q}^{2}
$$

In order to make equation (62) clearer let's consider the following numerical example:

$$
\sigma_{e l, T x}=0.015, \sigma_{o p t}=0.03, \sigma_{e l, R x}=0.015, \sigma_{\text {total }}=0 ., x_{l, e q}=(0,0.33,0.66,1)
$$

The optimum levels are calculated by replacing the values above in equation (62): 


$$
\begin{gathered}
x_{l_{\text {optimized }}}=\frac{0.015+0.015}{0.06} *\left(\begin{array}{c}
1 \\
0.66 \\
0.33 \\
0
\end{array}\right)+\frac{0.03}{0.06} *\left(\begin{array}{c}
1 \\
0.66 \\
0.33 \\
0
\end{array}\right)^{2} \\
x_{l_{\text {optimized }}}=0.5 *\left(\begin{array}{c}
1 \\
0.66 \\
0.33 \\
0
\end{array}\right)+0.5 *\left(\begin{array}{c}
1 \\
0.44 \\
0.11 \\
0
\end{array}\right)=\left(\begin{array}{c}
1 \\
0.55 \\
0.22 \\
0
\end{array}\right)
\end{gathered}
$$

In order to demonstrate the validity of this method a series of simulations were performed for a PAM-4 transmission at different values of $\sigma_{e l, T x}, \sigma_{o p t}$ and $\sigma_{e l, R x}$. In order to have a balanced comparison the value of $\sigma_{\text {total }}$ is set to 0.06 for all of the test cases. The top and bottom levels are set to 0 and 1 respectively. The value of the second level is varied between 0.02 and 0.35 and the value of third level is varied between 0.36 and 0.69 . The results of this simulation are displayed below.
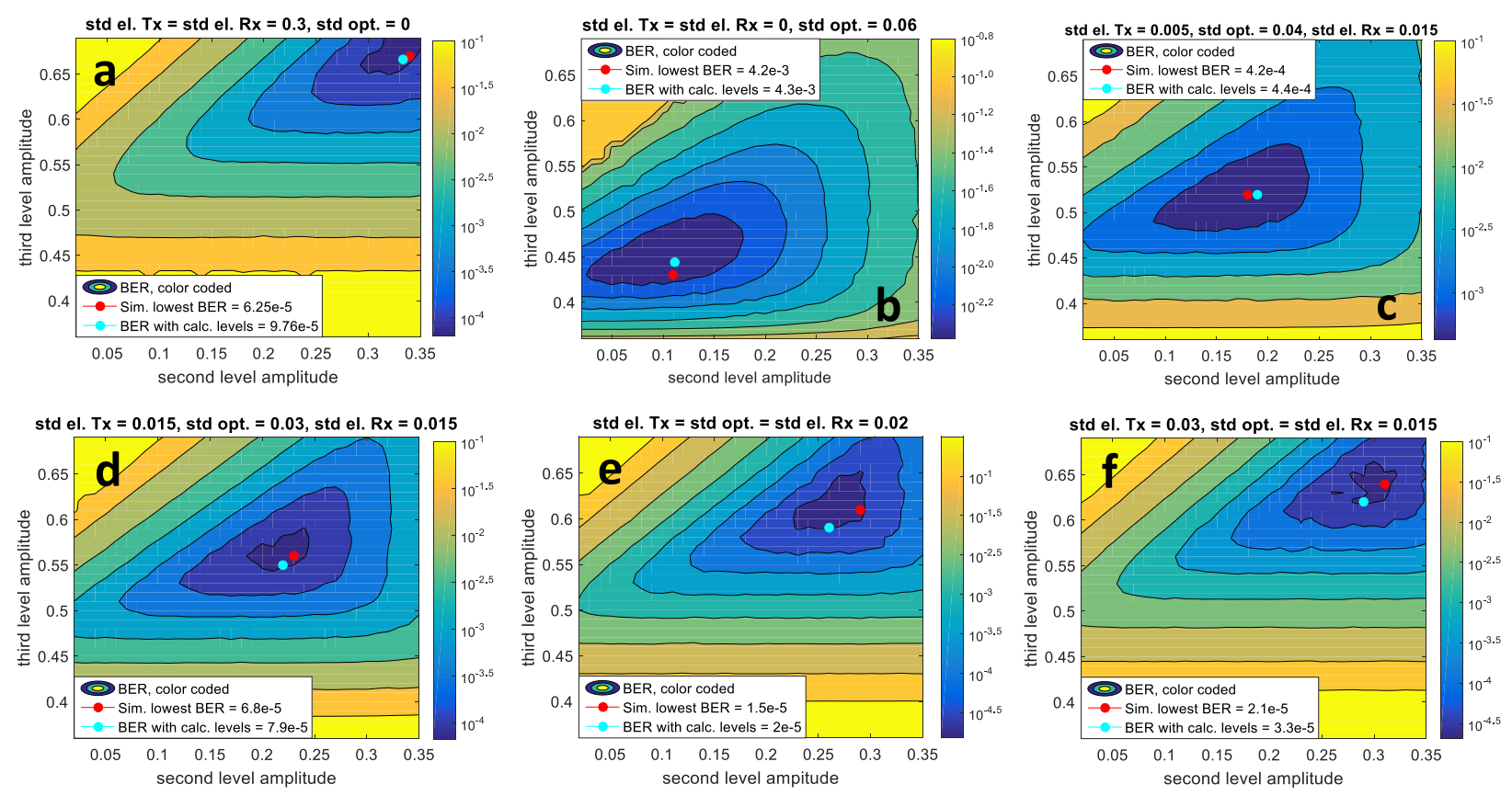

Figure 4.8. BER in relation with second and third level amplitude for the following scenarios: $\sigma_{e l, T x}=0.03, \sigma_{o p t}=0, \sigma_{e l, R x}=0.03(a), \sigma_{e l, T x}=0, \sigma_{\text {opt }}=0.06, \sigma_{e l, R x}=0.015(b), \sigma_{e l, T x}=0.005, \sigma_{o p t}=0.04$, $\sigma_{e l, R x}=0.015(c), \sigma_{e l, T x}=0.015, \sigma_{o p t}=0.03, \sigma_{e l, R x}=0.015(d), \sigma_{e l, T x}=0.02, \sigma_{o p t}=0.02, \sigma_{e l, R x}=0.02(e)$, $\sigma_{e l, T x}=0.03, \sigma_{o p t}=0.015, \sigma_{e l, R x}=0.015(f)$. The red dots indicate optimum levels from simulation and the cyan dots show the levels calculated with equation (62)

It can be observed in Figure 4.8 that the levels calculated with the method proposed here come very close to the absolute optimum values obtained by simulation (both in terms of amplitude levels and BER). The small difference between simulation and the calculated values is due to the fact that the modulation levels after modulator and after PD are already influenced by noise. This difference is quite small and can be ignored. While the PAM-4 modulation was employed in these 
simulations for simplicity, the technique described above can be extended also for higher order modulations with the same outcomes. In the case of OOK which has only two levels, geometrical constellation shaping is of course inapplicable.

The simulations above assumed an EML modulator. In the case when a MZM is used instead, the optimization process changes slightly. The amplitude transfer function of the MZM is no longer the square root of $x$, but instead is equal to the sinus function. If the whole modulator range is employed then equation (55) can be rewritten for the MZM case as:

$$
u=\sin \left(\left(x+n_{e l, T x}\right) * \frac{\pi}{2}\right)
$$

Given equation (63) then equation (62) becomes:

$$
x_{l_{\text {optimized }}}=\frac{\sigma_{\text {el,Tx }}}{\sigma_{\text {total }}} * x_{l, e q}+\frac{\sigma_{\text {opt }}}{\sigma_{\text {total }}} * \sin \left(x_{l, e q} * \frac{\pi}{2}\right)+\frac{\sigma_{e l, R x}}{\sigma_{\text {total }}} *\left[\sin \left(x_{l, e q} * \frac{\pi}{2}\right)\right]^{2}
$$

The sinus function is quite close to the square root one when the electrical signal is modulated properly, thus when a more simple approach is needed the transfer function of the MZM can be approximated with that of the EML such that equation (66) becomes the same as (62).

In order to demonstrate the validity of this approach in real life an experiment was devised, as described further in this paragraph. PAM-4 has become very popular in applications such as high speed data center interconnects [15], [70], [9] where small, cheap, reliable, and low power consumption transceivers are preferable. This is why this modulation format is chosen in this experiment, where an inter-datacenter single wavelength transmission is being emulated. MATLAB is used to generate the PRBS, map it to the PAM-4 symbols and then generate three types of constellation: a regular ESL PAM-4, a PAM-4 with levels which are optimized for optical noise mitigation, also referred to here as squared equally spaced levels (s-ESL) and a PAM-4 with levels optimized with equation (62) which is called here optimally spaced levels (OSL) PAM-4. The electrical signal is generated by means of AWG. The signal is then amplified and sent to a MZM which generates the optical signal. Due to the varying power of the three modulation schemes the bias of the modulator is adjusted to ensure the desired levels after receiver. ASE noise is generated by an EDFA and added to the signal. Experiments were carried out for the BTB scenario and for link lengths of 5 and $10 \mathrm{~km}$. After transmission an OBF removes the out-of-band noise. The optical signal is received by a PD and the input power is controlled by a variable optical attenuator (VOA) and kept at a constant value of $-2 \mathrm{dBm}$. The electrical eye diagrams of the three PAM-4 versions can be seen in Figure 4.9. Keep in mind that the levels appear inverted after the receiver (for this particular $\mathrm{PD}$, it is not a general rule). After the $\mathrm{PD}$ the signal is sampled by a real-time oscilloscope at a rate of 100 GSamples/sec. The offline data is then processed in MATLAB. Firstly, timing recovery is performed, followed by the automatic gain controller (AGC) and equalization which consists of a second order Volterra filter with 11 taps for the linear part and three taps for the nonlinear part. The linear part of a Volterra filter is the same as a FIR filter. 
The nonlinear part includes the kernels $\mathrm{x}^{2}(2), \mathrm{x}(1) \mathrm{x}(2)$, and $\mathrm{x}(2) \mathrm{x}(3)$. The last two kernels improve performance when ISI is significant. At the end the Gray BER is calculated in order to evaluate performance.

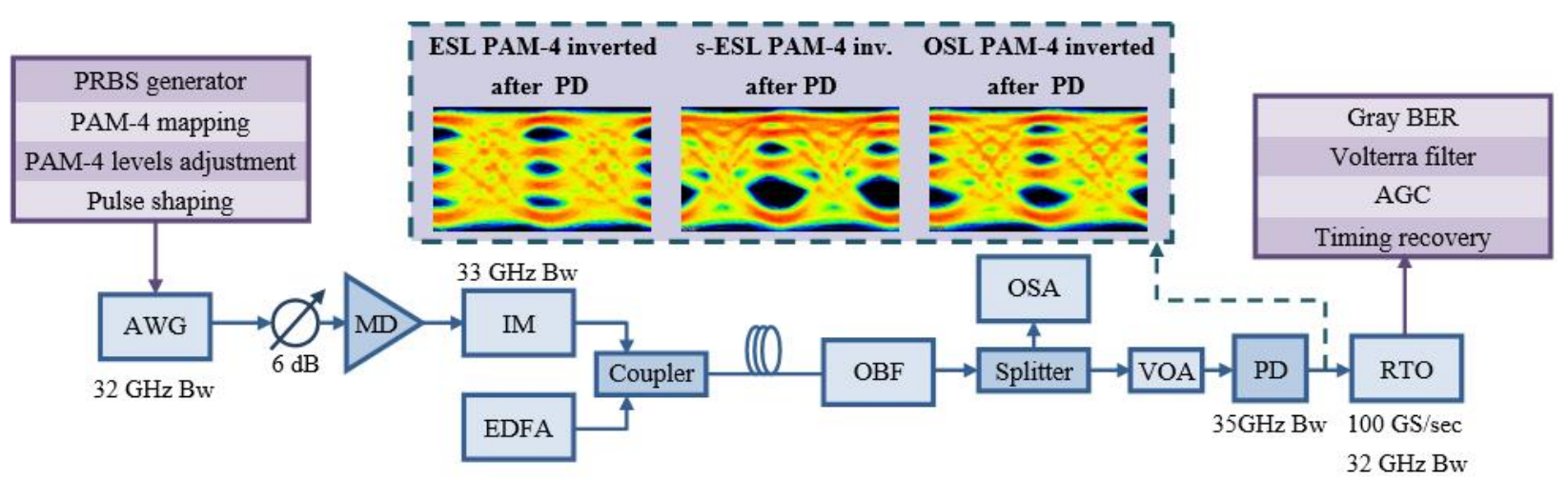

Figure 4.9. Experimental setup for testing the effects of BER performance of geometrical constellation shaping

While the ESL and s-ESL are straightforward to generate, in the case of OSL some information about the noise present in the system is required. First of all, we must consider that bandwidth limitations will interfere with the accuracy of OSL calculation. That is why high bandwidth components and a baud rate of $28 \mathrm{GBaud}$ are used. Then a transmission where no ASE noise is added is performed in order to estimate the electrical noise. From the histogram of the captured data the standard deviation of the electrical noise can be computed (i.e. after the equalizer so the influence of bandwidth limitations is mitigated). For this particular experimental setup the electrical noise $\sigma_{e l}$ calculated from the received histogram after the Volterra filter is equal to 0.021 when the amplitude of the signal is normalized to 1. The system in Figure 4.9 will introduce other impairments besides noise, such as reflections and nonlinear effects. Because of this the measurement of the electrical noise is not $100 \%$ accurate. The electrical noise is still the dominant effect so the measurement can be considered accurate enough for our purposes.

The next step is to calculate the standard deviation of the optical noise. For this purpose an EDFA is used to generate ASE noise and the OSNR is measured with the help of a spectrum analyzer. For this measurement an OSNR of $25 \mathrm{~dB}$ was chosen. Once we have the OSNR value the standard deviation of the noise can be easily obtained using the following equations:

$$
\begin{gathered}
\text { OSNR }[d B]=10 \log _{10}\left(\frac{P_{\text {signal }}}{\sigma_{\text {opt }}^{2}}\right) \\
\sigma_{\text {opt }}=\sqrt{\frac{P_{\text {signal }}}{10\left(\frac{\text { OSNR[dB] }}{10}\right)}}
\end{gathered}
$$

When the amplitude of the PAM-4 signal is normalized to 1 , the modulation levels are $0,1 / 3,2 / 3$ and 1 , resulting in a signal with the power: 


$$
P_{\text {signal }}=\frac{\sum_{i=1}^{N_{\text {levels }}} l_{i}^{2}}{N_{\text {levels }}}
$$

where $N_{\text {levels }}$ is the number of levels of the modulation and $l_{i}$ is the amplitude of the $i^{\text {th }}$ levels. Given equations (66) and (67) and the OSNR of $25 \mathrm{~dB}$ the value of $\sigma_{\text {opt }}$ is equal to 0.035 . Based on equations (61) and (62) the OSL can be calculated as:

$$
O S L=\frac{0.021}{0.056} *\left(\begin{array}{c}
1 \\
0.66 \\
0.33 \\
0
\end{array}\right)+\frac{0.035}{0.056} *\left(\begin{array}{c}
1 \\
0.66 \\
0.33 \\
0
\end{array}\right)^{2}=\left(\begin{array}{c}
1 \\
0.53 \\
0.2 \\
0
\end{array}\right)
$$

The values for the ESL and s-ESL are already known (see Table 3 below). Experiments with the three PAM-4 constellations are performed for BTB, $5 \mathrm{~km}$ and $10 \mathrm{~km}$ scenarios, using the setup from Figure 4.9. For the BTB case the results of processing the offline data are displayed in Figure 4.10. When comparing ESL with s-ESL it can be observed that although at low OSNR s-ESL can improve performance, when going to BERs below $10^{-3}$ ESL outperforms s-ESL, and the difference between the two grows larger as the BER value drops. This makes sense since s-ESL PAM-4 is optimized for optical noise and as OSNR rises the value of $\sigma_{\text {opt }}$ drops and the influence of the electrical noise grows. The best BTB performance is obtained when OSL is used. OSL shows an OSNR gain of $2 \mathrm{~dB}$ at a BER of $10^{-3}$ and of roughly $2.5 \mathrm{~dB}$ at BERs of $10^{-4}$ or lower, when comparing with ESL PAM-4. In Figure 4.10 results for ESL and OSL PAM-4 are shown in the presence of residual CD. It is assumed that either a dispersion compensation module (DCM) or DCF is employed to compensate for dispersion, while the residual CD is emulated by adding 5 and $10 \mathrm{~km}$ of standard fiber. The same Volterra filter as in the BTB case is employed here. Due to the poor performance shown in BTB the s-ESL is not considered anymore for the 5 and $10 \mathrm{~km}$ scenarios. In the $5 \mathrm{~km}$ transmission, OSL outperforms ESL by about $2 \mathrm{~dB}$ in terms of OSNR, similar to the BTB scenario. When increasing the fiber length to $10 \mathrm{~km}$ we can see that the OSL OSNR gain decreases down to around $1.1 \mathrm{~dB}$. At this transmission length $\mathrm{CD}$ becomes the major limiting factor and the Volterra filter can no longer properly compensate it, explaining the deteriorating OSL PAM-4 performance. At $15 \mathrm{~km}$ both OSL and ESL fail to achieve BERs below $10^{-3}$. The results presented in Figure 4.10 demonstrate the potential of geometrical shaping in IMDD systems. Considering its very low implementation complexity the OSL PAM-4 can help ameliorate DSP and FEC requirements, thus reducing the overall cost and power consumption of short-reach optical transmissions.

Table 3. The experimental normalized amplitude values for ESL, s-ESL and OSL PAM-4

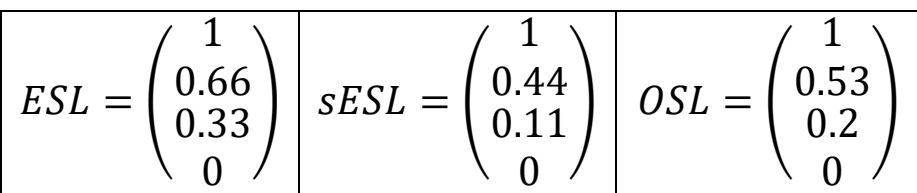



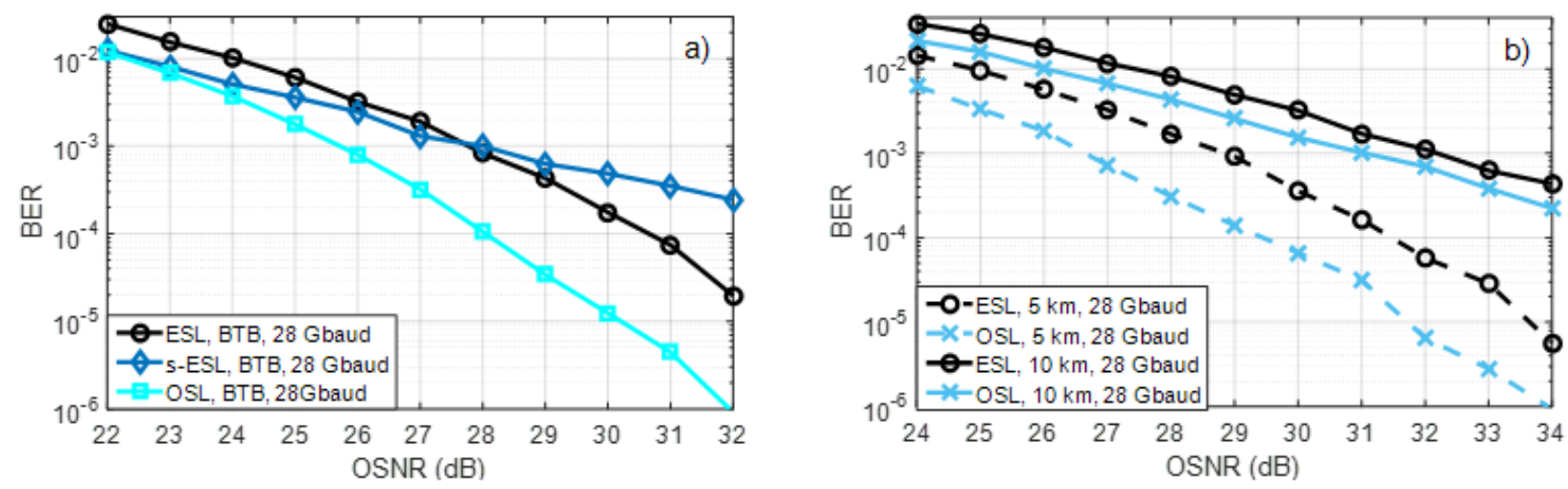

Figure 4.10. Experimental performance of ESL, s-ESL and OSL PAM-4 for BTB (a) and of ESL and OSL for 5 and $10 \mathrm{~km}$ links. Baud rate $=28$ GBaud

The OSL PAM-4 has been generated for an OSNR of $25 \mathrm{~dB}$ and its levels are slightly different for other OSNR values. Of course the amplitude levels can be optimized for each OSNR, however for simplicity the OSL calculated at $25 \mathrm{~dB}$ OSNR are used for the whole OSNR range of the experiments presented in this chapter (see Figure 4.10).

The classic PAM-4 constellation is not optimized from a geometrical point of view for IM-DD optical transmissions in the presence of ASE noise. A PAM-4 constellation with non-uniformly spaced levels which improves the noise tolerance of an optical system is proposed in this chapter. High OSNR gains of $1.5 \mathrm{~dB}$ or more are shown in simulation, while in experiments gains of around $2 \mathrm{~dB}$ are demonstrated in BTB and $5 \mathrm{~km}$ transmissions and of $1.1 \mathrm{~dB}$ for a $10 \mathrm{~km}$ transmission.

\subsubsection{Geometrical shaping for mitigating the effects of chromatic dispersion}

In specific scenarios geometrical constellation shaping can be employed to indirectly reduce the influence of $\mathrm{CD}$. The method presented in this section revolves around the use of PAM-4 with an IQ-MZM. Let's introduce the PAM-4 generation process which can be done in two ways when using an IQ-MZM. First approach is to directly generate the PAM-4 modulation at the output of the DAC. In the experiments presented in this section a BPG is employed. Thus, to generate the PAM-4 signal, two PRBS streams (i.e. OOK signal) are generated on two separate channels of the BPG. One of the streams is attenuated by $6 \mathrm{~dB}$ compared to the other, such that its power is halved. The two OOK signals are then sent to a combiner which adds them together, generating a classic PAM-4 modulation at its output. The electrical signal is then connected to the $I$ branch of the modulator, while the Q branch input remains empty, as shown in Figure 4.11(a). The optical eye diagram of the PAM-4 signal at the output of the modulator when the modulation is generated as described above can be seen in Figure 4.12(a). 
PRBS

Generator

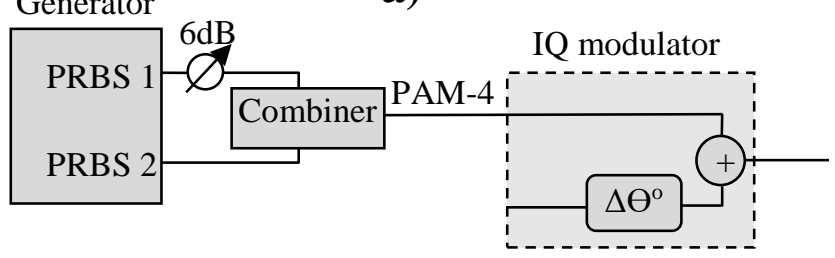

PRBS

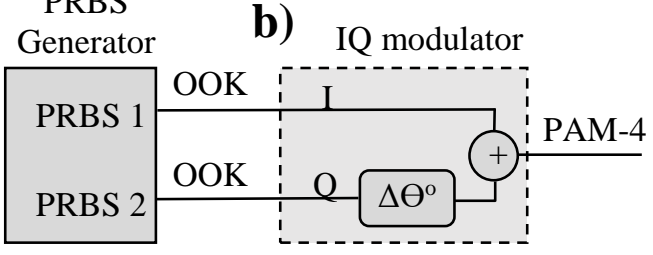

Figure 4.11. Generation of optical PAM-4 with a BPG and IQ-MZM using just the I branch of the modulator (a) or using both the I and the $Q$ branches $(b)$
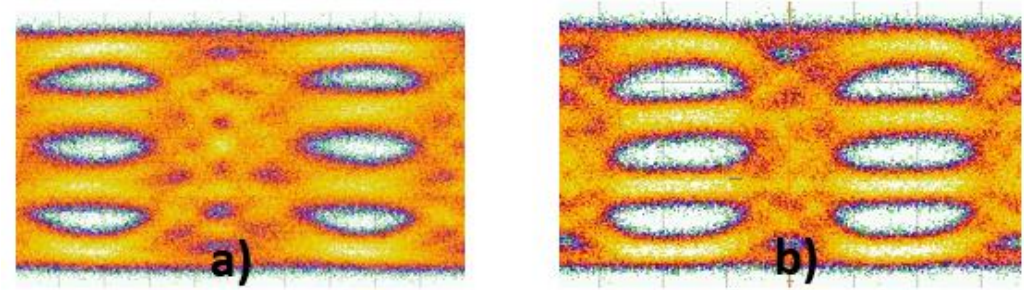

Figure 4.12. Optical eye diagram at the output of the MZM, when one branch is employed to generate PAM-4 (a) and when two branches are employed ( $b$ )

The second approach to generating the PAM-4 signal is to use the IQ-MZM to generate the four levels. Just like with the first method, two PRBS stream are outputted from the BPG. This time however they are not attenuated and no combiner is employed. One stream is connected to the $I$ branch of the MZM and the other to the $Q$ branch, as described in Figure 4.11(b). The optical eye at the output of the MZM is shown in Figure 4.12(b). Compared with the eye generated by the previous approach, the eye generated by this method is more open, indicating better performance. Thus, the second-generation process should be used.

If the optical noise is considered negligible then ESL PAM-4 is desired at both transmitter and receiver side. This implies that the optical power levels should also be equally spaced. In order to obtain equidistant power levels at the output of the IQ modulator, the phase bias of the Q branch needs to be $\pi / 2$ and the amplitude of one of the NRZ-OOK streams has to be $\sqrt{2}$ times larger than the other. Figure 4.13(a) shows the resulting constellation. It can be seen that the constellation points are not on the same axis, meaning that the signal is modulated in both intensity and phase domains. In an IM-DD system the phase modulation introduces chirp, which causes additional ISI when the signal is affected by CD. To counter this phenomenon, the phase bias between the two branches of the modulator must be set to zero. By doing this, it becomes impossible to generate an optical PAM-4 signal with ESL and thus the unequally spaced levels (USL) are obtained. In a BTB scenario this approach will provide worse BER than the equidistant levels PAM-4 for the same SNR requirements. However, when propagating through SSMF, the performance degradation is smaller in the case of USL PAM-4 and after a certain link distance it will provide better BER than its counterpart [69]. 


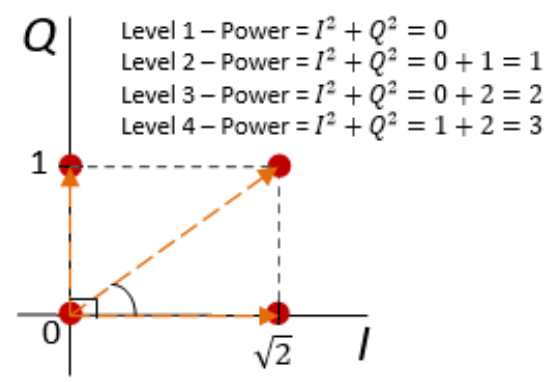

a)

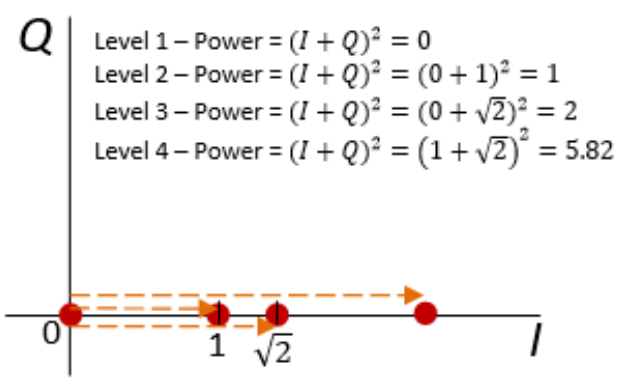

b)

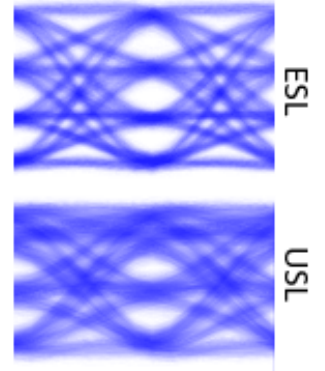

c)

Figure 4.13. The constellation of ESL PAM-4 (a) and of USL PAM-4 (b) and the corresponding eye diagrams at the receiver side for a BTB transmission (c)

In order to demonstrate the benefits of USL PAM-4 an experiment was implemented. The block diagram of this experimental setup is displayed in Figure 4.14. A BPG is used to generate two NRZ-OOK signals with the baud rate of 28 GBaud. One channel is attenuated by $6 \mathrm{~dB}$ more than the other $(\mathrm{Ch} 1=16 \mathrm{~dB}, \mathrm{Ch} 2=10 \mathrm{~dB})$. After a $24-\mathrm{dB}$ amplifier, the two signals will have the appropriate peak-to-peak voltage $(2 \mathrm{~V}$ and $1 \mathrm{~V})$ in order to operate the MZM in the linear region. The resulting constellations of the ESL and USL PAM-4 are shown in Figure 4.13(a) and (b). After passing through the SSMF fiber, the PAM-4 signal is detected by an InGaAs photodiode and sampled by a real-time scope at 50 GSamples/s. The PD employed here does not have a TIA, thus it requires high input optical power (i.e. 5-7 dBm). Since the power at the output of the MZM is of only $3 \mathrm{dBm}$ and the SSMF also attenuates the optical power (around $0.35 \mathrm{~dB} / \mathrm{km}$ ), an EDFA is necessary before the receiver.

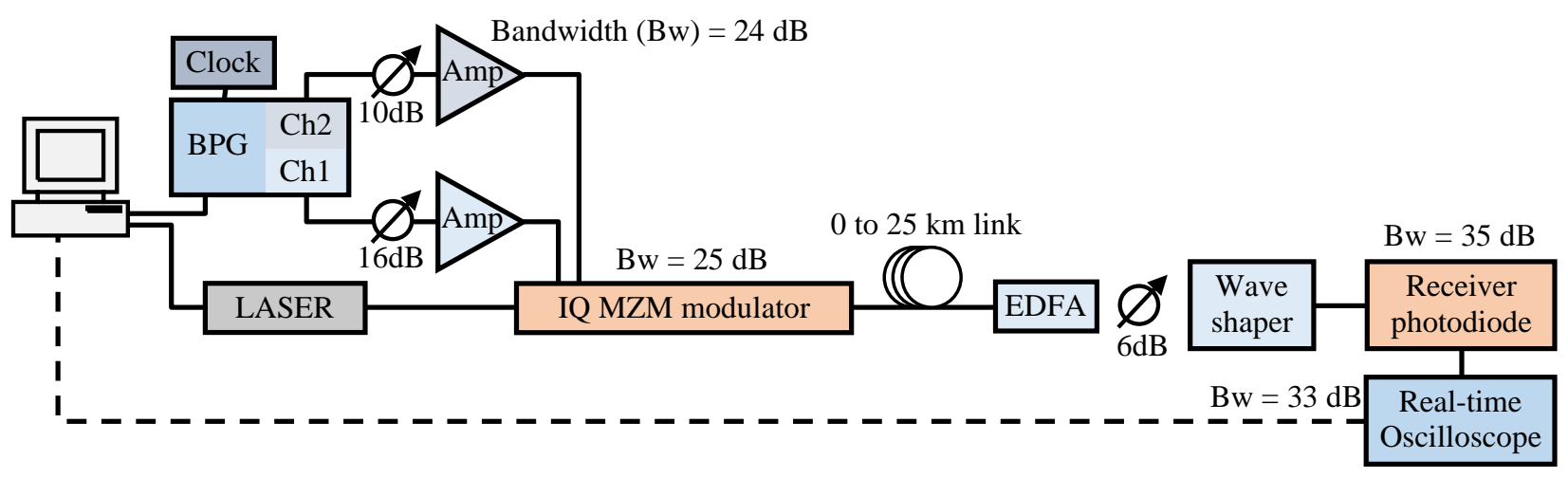

Figure 4.14. Experimental setup for testing USL PAM-4 CD mitigation capabilities

The performance of the two PAM-4 modulation schemes evaluated at link lengths of up to $25 \mathrm{~km}$ is presented in Figure 4.15(a), where a 16-state M LSE equalizer has been used. It can be seen that for short transmission distances of up to $8 \mathrm{~km}$ the equidistant levels PAM-4 performs better, due to its higher noise tolerance. However, for links of $10 \mathrm{~km}$ or longer, this scheme cannot achieve BER below the FEC threshold of $4 \times 10^{-3}$ (7\% Super-FEC). On the other hand, the unequally spaced levels modulation scheme is performing better after $8 \mathrm{~km}$ and can extend the transmission length 
to $25 \mathrm{~km}$. The eye diagrams in Figure 4.15(b) also show the difference between received signals affected by chirp (left side) and signals that are chirp free (right side).

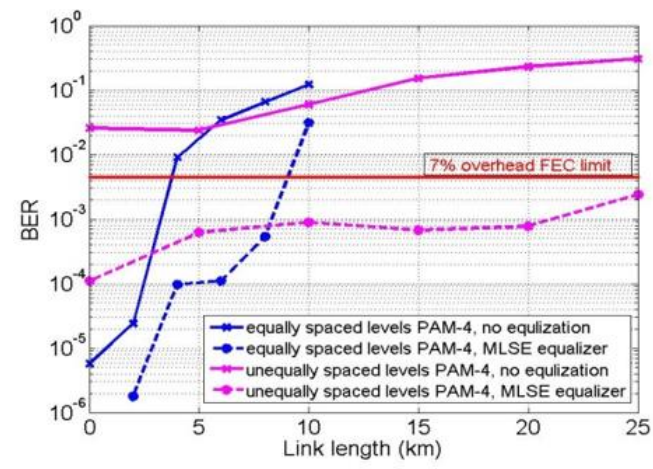

a)

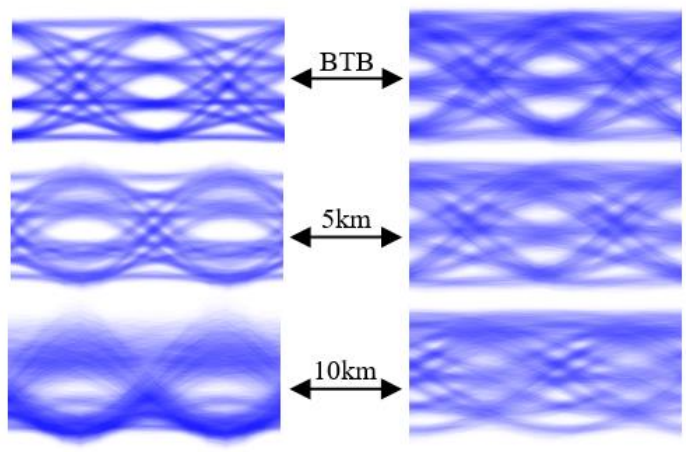

b)

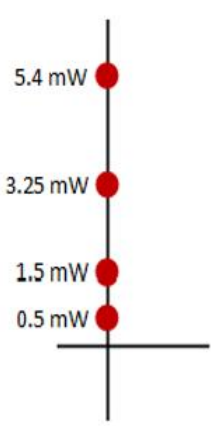

c)

Figure 4.15. Performance comparison between equally spaced levels (blue) and unequally spaced levels (magenta) PAM-4 (a), received eye diagrams for equally spaced levels (left column) and unequally spaced levels (right column) PAM-4 after 0, 5 and $10 \mathrm{~km}(\mathrm{~b})$ and the unequally spaced levels PAM-4 constellation with corresponding optical power levels (at the receiver the levels will be inversed) (c)

In this chapter the disruptive effects of the chirp introduced by an IQ modulator used for generating PAM-4 after propagating through SSMF are presented. A novel method based on unequally spaced levels that solves the chirp problem is proposed. The advantages and disadvantages of this solution are experimentally demonstrated and it is shown here that transmission lengths of up to $25 \mathrm{~km}$ at $56 \mathrm{~Gb} / \mathrm{s}$ are possible using this USL PAM-4 modulation scheme, compared with just up to $8 \mathrm{~km}$ with traditional ESL PAM-4.

\subsection{Probabilistic constellation shaping}

Probabilistic shaping is investigated mainly in coherent systems although this concept is also studied in non-coherent systems with four and eight-level PAM together with soft decision FEC [71]. The main reason is that next-generation high-speed data center interconnects will utilize optical fiber links with small, inexpensive, reliable, and low power consumption transceivers. The PAM-4 modulation format has become a promising candidate for such applications. In optically amplified links with amplifiers working in the saturation regime, PAM probabilistic shapers with very low complexity are preferable and can be used to improve OSNR performance, which is very critical for multilevel PAM formats. One approach for a distribution matcher design that transforms independent Bernoulli distributed input bits into output symbols with the specified distribution [72] comes from fixed-input fixed-output length transformation [73]. To achieve the designed probabilities, the distribution matchers require very long input sequence, which is unacceptable in practical systems. Some simplifications are already proposed in [74], [75]. In general, the distribution matchers seem to be very complicated and introduce too much latency.

In this chapter a simplified distribution matcher based on Hadamard matrix with negligible complexity and latency is proposed. The target is to improve the OSNR performance by increasing 
baud rate and exploiting the available system bandwidth. This can be compared with FEC which introduces additional redundancy. If a hard decision FEC with 7\% redundancy is used, in order to improve OSNR performance by $1 \mathrm{~dB}$ we have to implement much stronger and more complex FEC with almost $10 \%$ higher redundancy. It is shown here, both in simulations and experiments, that this can be easily done by shaping PAM-4 levels with less redundancy in IM-DD systems.

The main goal of the shaper is to increase the probabilities of lower PAM-4 levels (or to decrease the power) without changing the constellation. The optimum probabilities are calculated based on the OSNR value and Maxwell-Boltzmann density functions [76]. To generate a binary sequence with a specific probability of zero, several candidates are constructed and the most appropriate one is selected. In order to explain how the probabilistic shaping is done let's first assume that a NRZOOK sequence is transmitted. This bit sequence is split into multiple blocks with the same length. This length is denoted here by $M$. The blocks are sent to the probabilistic shaper which adds $K$ redundancy bits. To better explain how the shaper works let us assume a numerical example where $M=4$ and $K=3$. The encoder builds $2^{K}$ candidates which are based on the Hadamard matrix (square matrix with orthogonal rows). The $M \times M$ Hadamard matrix is constructed with the following elements:

$$
\text { Hadamard matrix }=\left(\begin{array}{llll}
0 & 0 & 0 & 0 \\
0 & 0 & 1 & 1 \\
0 & 1 & 0 & 1 \\
0 & 1 & 1 & 0
\end{array}\right), \text { Inverted Hadamard matrix }=\left(\begin{array}{llll}
1 & 1 & 1 & 1 \\
1 & 1 & 0 & 0 \\
1 & 0 & 1 & 0 \\
1 & 0 & 0 & 1
\end{array}\right)
$$

Four more rows are added, which are obtained by the inversion of the existing rows: '1111', '1010', '1100', and '1001'. The input sequence is then scrambled by each row and $K$ redundant bits (natural binary coding) are added to each candidate. If, for example, the bit sequence at the input of the shaper is ' 1111 ' then the candidates (i.e. codewords) are:

$$
\text { candidates } \left.=\left[\begin{array}{llll}
0 & 0 & 0 & 0 \\
0 & 0 & 1 & 1 \\
0 & 1 & 0 & 1 \\
0 & 1 & 1 & 0 \\
1 & 1 & 1 & 1 \\
1 & 1 & 0 & 0 \\
1 & 0 & 1 & 0 \\
1 & 0 & 0 & 1
\end{array}\right] \circ\left[\begin{array}{llll}
1 & 1 & 1 & 1 \\
1 & 1 & 1 & 1 \\
1 & 1 & 1 & 1 \\
1 & 1 & 1 & 1 \\
1 & 1 & 1 & 1 \\
1 & 1 & 1 & 1 \\
1 & 1 & 1 & 1 \\
1 & 1 & 1 & 1
\end{array}\right],\left[\begin{array}{lll}
0 & 0 & 0 \\
0 & 0 & 1 \\
0 & 1 & 0 \\
0 & 1 & 1 \\
1 & 0 & 0 \\
1 & 0 & 1 \\
1 & 1 & 0 \\
1 & 1 & 1
\end{array}\right]\right]=\left[\begin{array}{lllllll}
1 & 1 & 1 & 1 & 0 & 0 & 0 \\
1 & 1 & 0 & 0 & 0 & 0 & 1 \\
1 & 0 & 1 & 0 & 0 & 1 & 0 \\
1 & 0 & 0 & 1 & 0 & 1 & 1 \\
0 & 0 & 0 & 0 & 1 & 0 & 0 \\
0 & 0 & 1 & 1 & 1 & 0 & 1 \\
0 & 1 & 0 & 1 & 1 & 1 & 0 \\
0 & 1 & 1 & 0 & 1 & 1 & 1
\end{array}\right],
$$

where $^{\circ}$ represents the Hadamard product (element-wise product of two matrices with the same dimensions). The number of zeros for each candidate is counted and the codeword with the most zeros is selected. In this case the selected codeword is '0000100'.

By employing the method described in the previous paragraph the number of codewords is limited by the dimensions of the Hadamard matrix (i.e. $2 \times M$ ). This also means that the maximum value of $K$ is restricted by $M$ : 


$$
2^{K} \leq 2 M \rightarrow K \leq \log _{2}(2 M), \quad K \in \mathbb{N}
$$

For a $K$ which is smaller than $\log _{2}(2 M)$ only the first $2^{K-1}$ rows of the Hadamard matrix and their inverse are taken into consideration. The larger the ratio $\frac{K}{M}$ is, the higher the probability of ' 0 ' can be. Figure 4.16(a) shows the probability of zeros for different values of $M$ and $K$. For the example presented above ( $M=4, K=3)$ the probability of ' 0 ' is 0.79 . If a lower probability is desired using the same parameters then the probabilistic shaper should choose the codewords with the highest number of zeros when below the desired probability and the codewords with the lowest number of zeros when above the desired probability. This way, the averaged probability will vary around the target value.
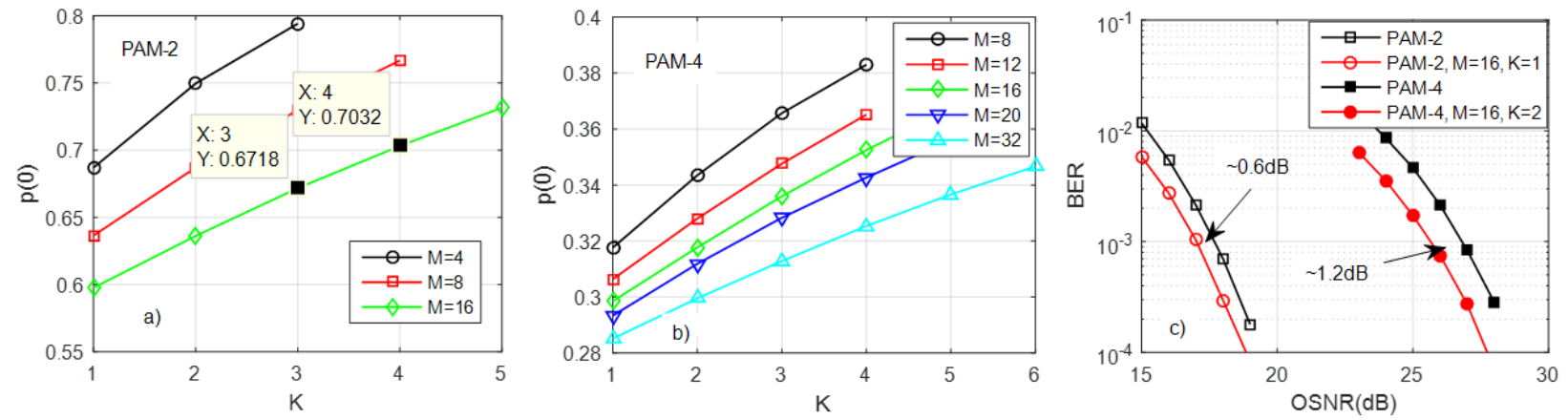

Figure 4.16. K vs. zero level probability for OOK (a) and for PAM-4 (b). Simulation of BER performance of OOK and PAM-4 with and without probabilistic shaping (c)

For the PAM-4 modulation two PRBS sequences are considered, one of them for the LSB and the other for the MSB. To keep the complexity low, the PAM-4 shaper controls only the MSB so that the first two levels have the same probability (probabilities of levels 1 and 2 and of levels 3 and 4 are equal). This simplified approach can be extended also to higher order modulations like PAM8 and PAM-16. The achievable probabilities for the first and second levels are shown in Figure 4.16(b). In systems with unlimited bandwidth, the probabilistic gain can be very large. However, in the real systems this is not the case. Therefore BTB, 28GBaud (net rate) OOK ( $M=16$ and $K=1$ ) and PAM-4 $(M=16$ and $K=2)$ systems are simulated with the total bandwidth of $20 \mathrm{GHz}$ and realistic IM-DD system parameters (noise, chirp, extinction ratio). The simulation results shown in Figure 4.16(c) indicate a gain at BER $=10^{-3}$ of 0.6 and $1.2 \mathrm{~dB}$ for OOK and PAM-4, respectively.

To prove the benefits of probabilistic shaping for IM-DD transmissions an experiment was devised. Since probabilistic shaping helps mitigate the effects of ASE noise, it is advisable to limit ISI and thus components with high bandwidth are employed, as seen in Figure 4.17. In MATLAB a $2^{16}$ length PRBS is generated. Then a distribution matcher (as described in this section) is used to generate the desired symbol probabilities. Input sequences of $M=16$ are considered and $K$ 's equal with 1,2, 3 or 4 are used. We refer to these probability shaping's as $\operatorname{PS}(M, K)$. After the distribution matcher the bits are mapped to PAM-4 symbol. The target is to achieve equidistant PAM-4 levels after receiver and thus a level adjustment is executed at the transmitter side. Next pulse shaping is performed and the data is then loaded into an AWG. The electrical signal from 
the AWG is amplified by $18 \mathrm{~dB}$ and then a single-drive MZM is employed to generate the optical signal. An EDFA is used as an ASE noise source and a coupler is employed to add the noise onto the optical signal. Experiments are performed for the BTB scenario and with up to $15 \mathrm{~km}$ worth of residual CD. After the link an OBF filters out the out-of-band noise. The optical power is controlled with the help of a VOA and is kept always at $-2 \mathrm{dBm}$. A high bandwidth PD is employed to transfer the signal back into the electrical domain and a real-time oscilloscope is used to capture the data at a rate of $100 \mathrm{GSamples/sec.} \mathrm{Timing} \mathrm{recovery} \mathrm{is} \mathrm{performed} \mathrm{on} \mathrm{the} \mathrm{offline} \mathrm{data,} \mathrm{followed}$ by the AGC and equalization. At the end, the Gray BER is calculated.

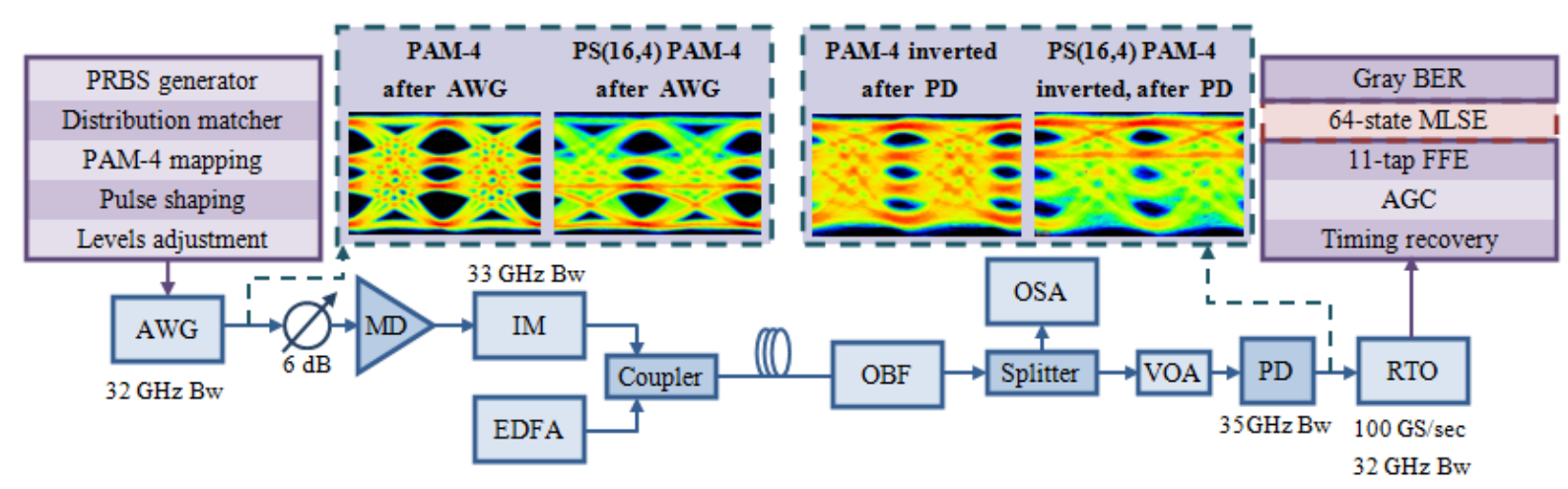

Figure 4.17. Experimental setup for testing the performance of probabilistic shaping
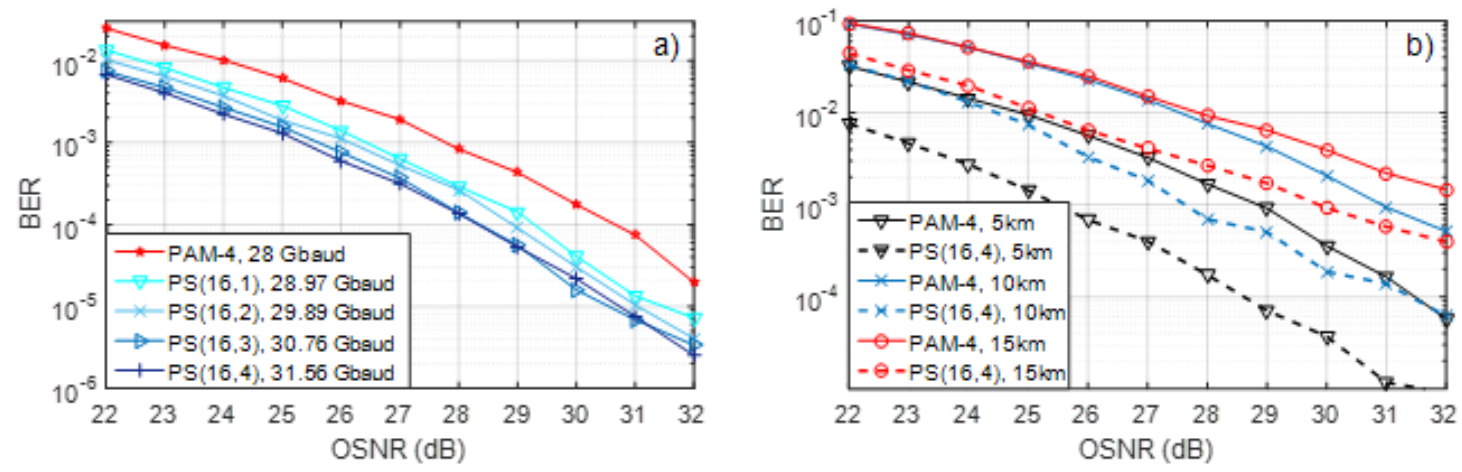

Figure 4.18. OSNR performance comparison between classic PAM-4 and PAM-4 with probabilistic shaping for: BTB, with an 11-tap FFE (a) and $5 \mathrm{~km}$ with 11-tap FFE, 10 and $15 \mathrm{~km}$ with 11-tap FFE plus 64-state MLSE (b)

In Figure 4.18 the experimental results are presented. First the performance of PAM-4 without and with probabilistic shaping is analyzed in the BTB scenario (Figure 4.18(a)). Four different values of $K$ (i.e. 1, 2, 3, and 4) are investigated. An 11-tap FFE is employed to remove any ISI the experimental setup might introduce. The weakest probabilistic shaping, PS(16,1), shows gains of around $1.3 \mathrm{~dB}$ in terms of OSNR, when compared with regular PAM-4. PS(16,2) and PS(16,3) increase the gain to approximately 1.7 and $2.4 \mathrm{~dB}$ respectively. Increasing redundancy further to $\mathrm{K}=4$ does not provide any further considerable gain, with PS $(16,4)$ improving the OSNR gain by roughly $2.5 \mathrm{~dB}$. The interactions between the components employed in the experiment add extra noise on top of the ASE noise. This effect could not be replicated accurately in our simulation, 
explaining why the experimental OSNR gain of probabilistic shaping is larger than the simulation gain. In Figure 4.18(b) the residual dispersion tolerance of probabilistically shaped PAM-4 is weighted against that of classic PAM-4. For this purpose the best performing probabilistic shaping in BTB was chosen (i.e. PS(16,4)). A transmission through DCF is assumed and the left-over CD is emulated by adding 5,10 , and $15 \mathrm{~km}$ worth of residual dispersion. In the $5 \mathrm{~km}$ scenario the difference between PAM-4 with and without shaping increases to around $3 \mathrm{~dB}$. When moving to 10 and $15 \mathrm{~km}$ the FFE is no longer sufficient to bring the BER below 10-3 and thus a 64-state MLSE is added. The OSNR gain provided by PS $(16,4)$ over normal PAM-4 is around $3 \mathrm{~dB}$ at 10 $\mathrm{km}$ and around $2.8 \mathrm{~dB}$ at $15 \mathrm{~km}$.

The experimental results shown here demonstrate the potential of probabilistic shaping in IM-DD transmissions. The high OSNR gains provided coupled with the low complexity of the distribution matcher can help to reduce the complexity of receiver DSP and of FEC, bringing down the overall complexity and power consumption of optical systems. This approach can be used with all multilevel PAM schemes. 


\section{Chapter 5 Partial response signaling}

As previously discussed, the continuous data traffic demand increase [77] is translated into a need for faster IM-DD optical systems, especially for the intra- and inter-datacenter networks. The $4 \lambda \times 10$ GBaud (i.e 40G systems) and 4 $4 \times 25$ GBaud (i.e. 100G systems) systems usually employ the NRZ-OOK modulation due to its ease of implementation, very low complexity (both for the modulation itself and the DSP algorithms) and high noise tolerance [78]. To accommodate the growing transmission speed demand, in the past few years the industry has been shifting from the $100 \mathrm{G}$ systems to the $400 \mathrm{G}(4 \lambda \times 100 \mathrm{GBaud})$. However, this increase in baud rate requirements has not been matched by the hardware, thus IM-DD optical systems have had to work with less and less bandwidth. As a result of this OOK cannot be used to the same effect for the 400G generation. That is why research focus has shifted to more spectrally efficient modulations such as PAM-4 [79], DMT [80] or CAP [12]. Out of these PAM-4 is the preferred choice due to its lower power consumption and complexity, when compared to the others. Since cost is a major concern when it comes to non-coherent transmissions, IM-DD systems require low-cost components. Typically, the bandwidth of the system is dictated by the bandwidth of the DAC, modulator and PD receiver, which stand at around $20 \mathrm{GHz}$ or higher. There are of course higher bandwidth components available, however these are usually either in-house solutions which are not commercially available or they are too expensive. The $100 \mathrm{Gbps}$ non-coherent transmissions have to work with an end-to-end 3-dB bandwidth of less than $15 \mathrm{GHz}$, meaning that even PAM-4 might not be enough. A solution to this problem is to use even higher order intensity modulations like PAM-8 or PAM-16 but these have very high SNR requirements which makes them impractical. Another solution, which is the main focus of this chapter, is to employ PRS techniques, in particular the DB signaling.

\subsection{Duobinary/polybinary signaling generation}

When a transmission system has a bandwidth less than half the symbol rate (less than the Nyquist frequency) it will be affected by ISI (i.e. the duration of one pulse is longer than the period of the signal, thus affecting neighboring pulses). In order to mitigate the effects of ISI introduced by bandwidth limitations some controlled ISI can be introduced, such that the spectral occupancy of the transmitted signal is reduced and thus less bandwidth is required. The most common form of PRS is referred to as DB [81], [82]. The basic idea behind it is to sum each transmitted symbol with its precursor neighbor, which will cause the spectral occupancy of the signal to be halved. 
There are two main approaches to the encoding/decoding of DB signals, the block diagrams of which are displayed in Figure 5.1. For the first method (see Figure 5.1(a)) a PAM signal is first created in the classic way (see Figure 4.1). After the symbols are generated a differential encoder is applied:

$$
b_{i}=\left(a_{i}-b_{i-1}\right) \text { modulo } N_{\text {levels }}, \quad i=1 \text { to } N \text {, }
$$

where $a_{i}$ are the symbols after the mapper, $b_{i}$ are the differentially encoded symbols and $N$ represents the number of transmitted symbols. Next the DB filter is applied, which is basically a $1+1 z^{-1}$ filter:

$$
c_{i}=b_{i}+b_{i-1}
$$

where $c_{i}$ are the transmitted DB symbols. A comparison between the regular PAM spectrum (i.e. spectrum of $a_{i}$ ) and the spectrum of the DB PAM (i.e. spectrum of $c_{i}$ ) can be seen in Figure 5.1(c). While the DB signal is far less vulnerable to the bandwidth limitations, it is still affected. When bandwidth of the system is around four times less than the baud rate, then transmission system itself can be used as a $1 z+1 z^{-1}$ filter thus removing the need for equation (71), helping improve the performance of DB signaling even further. At the receiver side the original PAM symbols can be recovered by doing a simple modulo operation:

$$
y_{i}=c_{i} \text { modulo } N_{\text {levels }},
$$

where $y_{i}$ is the decoded data and should be equal with $a_{i}$ and $N_{\text {levels }}$ is the number of levels of the modulation (e.g. for PAM-4 $N_{\text {levels }}=4$ ).
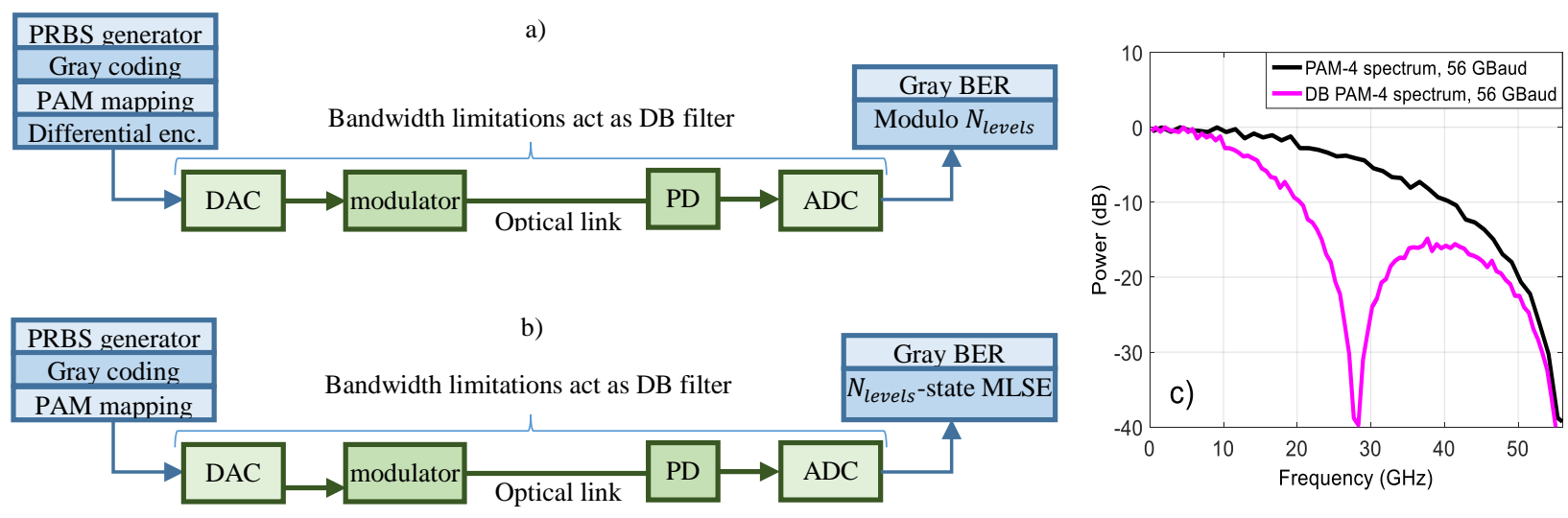

Figure 5.1. DB codec with differential encoding (a) and with MLSE decoding (b) and spectral comparison between PAM-4 and DB PAM-4 (c)

There is also the option to skip the differential encoder at the transmitter side, as shown in Figure 5.1(b). With this approach modulo $N_{\text {levels }}$ decoding is no longer viable, instead MLSE detection is needed. The memory length $L$ of the MLSE should be equal or larger than that of the length of the artificially introduced ISI (expressed in number of periods, $T$ ). In the case of DB, the length of the ISI is $1 T$, thus $L \geq 1$ resulting in a $N_{\text {levels }}$-state MLSE. 
The ISI can be extended to more than one symbol, resulting in a so-called polybinary (PB) signaling [82], [83]. In this case equations (70)-(72) become:

$$
\begin{gathered}
b_{i}=a_{i}-b_{i-1}-b_{i-2}-\ldots-b_{i-N_{T}} \\
c_{i}=b_{i}+b_{i-1}+\ldots+b_{i-N_{T}} \\
y_{i}=c_{i} \text { modulo } N_{\text {levels }}
\end{gathered}
$$

where $N_{T}$ is the number of periods affected by the inserted ISI. The main drawback of DB/PB modulation is that it increases the number of levels of the modulation which translates into increased noise sensitivity. This number of levels is determined by the number of levels of the initial modulation and the length of the artificially introduced ISI:

$$
\begin{gathered}
N_{\text {levels }, D B}=2 N_{\text {levels }}-1 \\
N_{\text {levels }, P B}=\left(N_{T}+1\right) N_{\text {levels }}-N_{T}
\end{gathered}
$$

If, for example, a DB PAM-4 signal is transmitted then the received signal has seven levels and if a PB PAM-4 signal with $N_{T}=3$ is transmitted then the received data have 13 levels. Due to the large increase in noise sensitivity, PB is rarely employed, DB being the preferred solution in heavily bandlimited IM-DD optical systems.

\subsection{Duobinary FFE equalization}

The structure of the FFE equalizer has been presented in chapter 3.2. In that chapter the training of the equalizer taps was also introduced. In the case of a DB signal the structure of the FFE stays the same. The only difference is that instead of detecting $N_{\text {levels }}$, now $2 N_{\text {levels }}-1$ (i.e. $N_{\text {levels,DB }}$ ) have to be detected. The same holds true for PB, instead of detecting $N_{\text {levels }}, N_{\text {levels,PB }}$ have to be detected. Thus equations (45)-(47) from chapter 3.2 can still be used here to describe the FIR filter and the training of the equalizer, the only difference being that $\bar{y}[n]$ now has $N_{\text {levels,DB }}$ (e.g. for PAM-4 $\bar{y}[n]$ has four levels while for DB PAM-4 it has seven levels).

The goal of this section is to determine if DB FFE can provide any gain over regular FFE and if so, in which scenarios does DB FFE perform better. For this purpose a series of experiments employing PAM-4 as modulation are performed. The block diagram of the experimental setup is shown in Figure 5.2. The modulated data is generated in MATLAB as described in chapter 4.1. This data is converted into an electrical signal with the help of a Fujitsu 8-bit DAC. The 3-dB bandwidth of the DAC is lower than $15 \mathrm{GHz}$ and its transfer function can be seen as inset to Figure 5.2. The amplitude of the signal at the output of the DAC is approximately $400 \mathrm{mV}$. However, the optimum amplitude required by the modulator is around $2 \mathrm{~V}$. As a result, a MD electrical amplifier is required to amplify the signal at the output of the DAC. In order to adjust the bias point a biasT is employed. The laser and the modulator are integrated together into a $20 \mathrm{GHz} 3-\mathrm{dB}$ bandwidth 
TOSA, which operates at $1300 \mathrm{~nm}$ wavelength. The optical link consists of SSMF. For this experiment the performance of non-DB and DB FFE is tested in a BTB scenario at baud rates ranging from 28 up to 56 GBaud. A VOA is added before the receiver in order to adjust the power of the optical signal. The conversion of the optical signal back into the electrical domain is done by a ROSA with differential output and a $3-\mathrm{dB}$ bandwidth of around $20 \mathrm{GHz}$. The ROSA comprises of a photodiode and a TIA. A $33 \mathrm{GHz}$ bandwidth real-time scope is used to capture offline data at a rate of $80 \mathrm{GSamples} / \mathrm{s}$. Offline an AGC is employed to remove the DC component of the received signal, being followed by the timing recovery. The FFE is trained to detect four or seven levels (i.e. detect PAM-4 or DB PAM-4). The equalizer is followed by a 4-state MLSE which can further improve performance and, in the DB case, detect the original four levels from the received data. In the end the Gray BER is calculated, allowing us to evaluate the performance of the optical transmission.

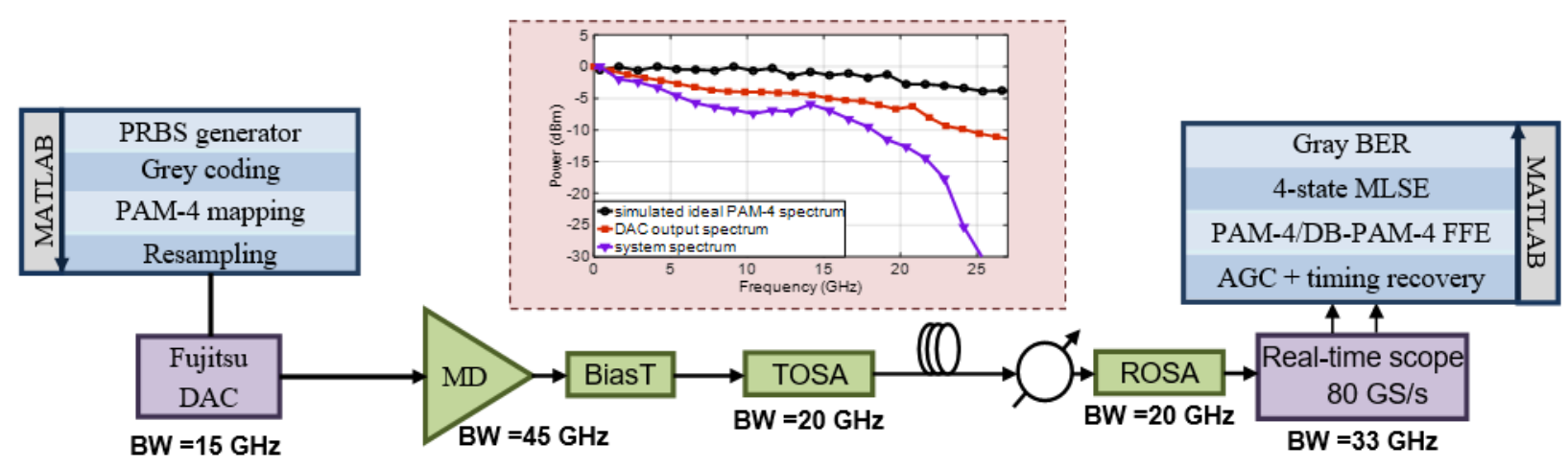

Figure 5.2. Experimental setup for testing DB FFE performance together with the spectrum of the DAC and system spectrums

The total 3-dB bandwidth of the experimental setup is lower than $5 \mathrm{GHz}$. Furthermore, the spectral power starts dropping very fast for frequencies higher than $15 \mathrm{GHz}$. The regular FFE tries to reconstruct the transmitted PAM-4 spectrum. If the system has a low bandwidth relative to the baud rate, the spectrum of the received signal might be closer to the DB PAM-4 spectrum than to the original PAM-4 spectrum. Thus, it should be easier for the equalizer to reconstruct the spectrum as a DB spectrum, resulting in better BER performance. Considering these considerations, it is expected that for the experimental setup in Figure 5.2 DB FFE should provide better results for high baud rates. By transmitting the same PAM-4 data at different baud rates we seek to determine if indeed DB equalization can outperform the classic FFE and, if yes, starting from which baud rate does $\mathrm{DB}$ equalization become superior to non-DB equalization. As stated before in this section of the thesis, a PAM-4 signal is transmitted BTB at the following baud rates: 28, 32, 36, 40, 44, 48, 52 and 56 GBaud. The sensitivity curves when using DB and non-DB FFEs are displayed in the figure below: 

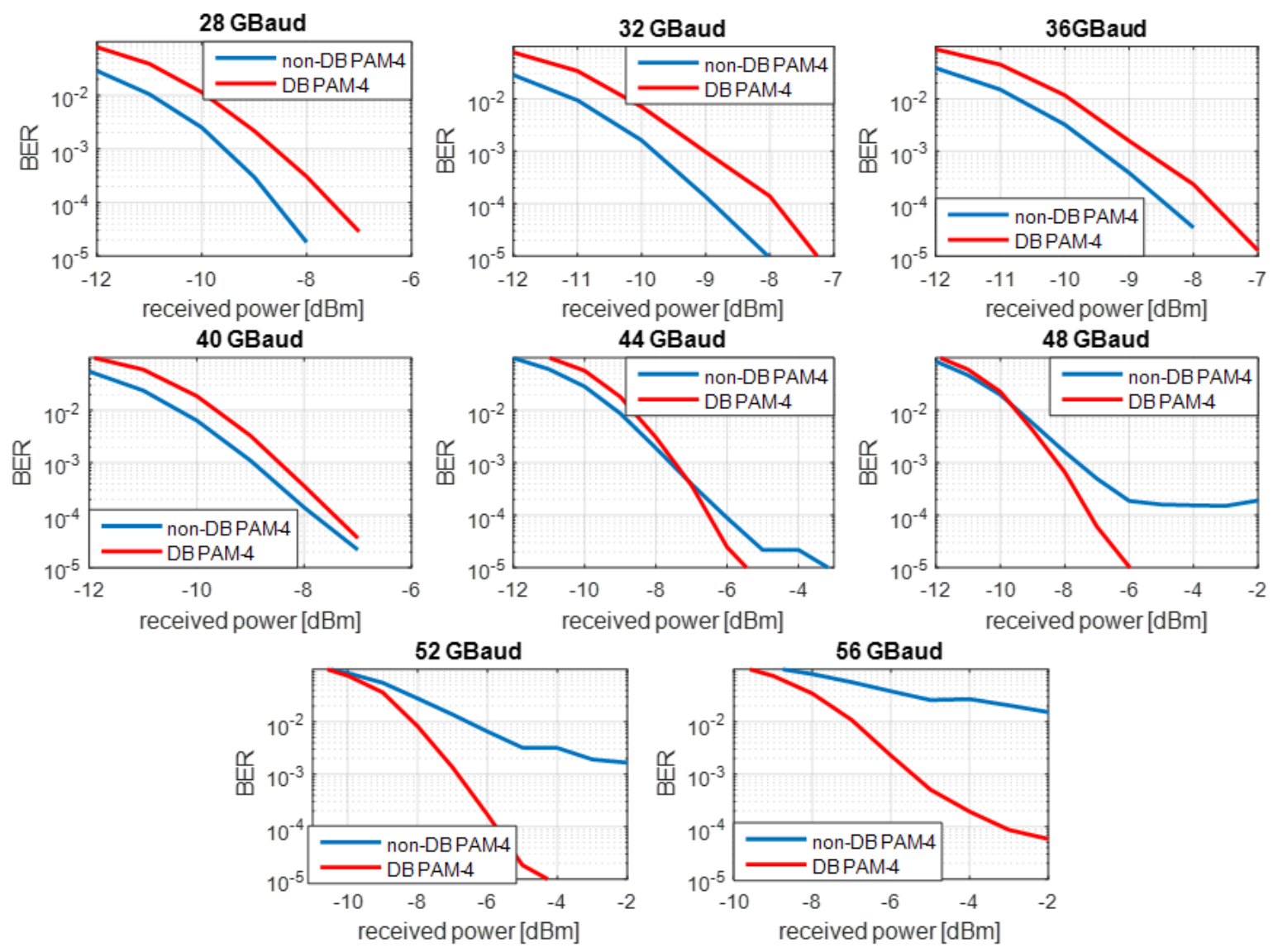

Figure 5.3. Performance comparison between classic PAM-4 and DB PAM-4 at baud rates from 28 to 56 GBaud

For the baud rates of 28, 32, 36 and 40 GBaud, the classic 4-level FFE approach provides better sensitivity than the 7-level DB FFE. However, it can be seen (in Figure 5.3) that the sensitivity gap between the two techniques gets smaller as the baud rate increase; if at 28 GBaud non-DB PAM-4 is roughly $1 \mathrm{~dB}$ better, at $40 \mathrm{GBaud}$ the difference between the two is reduced to less than half of $\mathrm{dB}$ for BERs below $10^{-3}$. The $44 \mathrm{GBaud}$ baud rate appears to be the transition point, where DB starts to outperform the non-DB approach. At higher baud rates the classic PAM-4 equalizer can no longer remove the error floor. This error floor rises with the transmission speed, thus if at 48 GBaud the BER floor stand at about $10^{-4}$, at 56 GBaud it is above $10^{-2}$. Transmission at 52 and 56 GBaud are not possible with regular the regular 4-level FFE approach at BERs below the KP4 FEC threshold of $3 \times 10^{-4}$. The DB FFE removes the BER floor the 48 and 52 GBaud transmissions and greatly increases the performance at baud rates higher than 44 GBaud.

It is experimentally proven in this section that DB signaling can indeed improve performance in bandlimited IM-DD systems and that it enables faster than Nyquist transmission rates. Since, for the most part, very high transmission rates are targeted in this work, DB is an essential DSP technique. 


\subsection{Low-cost PAM solutions for $100 \mathrm{Gbps} /$ lambda transmissions}

The short-reach non-coherent optical market is currently moving from the 100G WDM solutions to the 400G WDM solutions [84]. The preferred approach is to multiplex four wavelengths, with each wavelength carrying $100 \mathrm{Gbps}$ net rate. As discussed in previous chapters several options were considered for modulation but it seems like PAM-4 is preferred. That is why currently research efforts are focused on $100 \mathrm{Gbps} / \lambda \mathrm{IM}-\mathrm{DD}$ optical transmissions, mainly on reducing their cost and power consumption. In this section of the thesis a series of experiments for $100 \mathrm{Gbps} / \lambda$ systems using PAM are performed. The results are presented and analyzed here.

Let us start by introducing the experimental setup (Figure 5.4). Since it has been established that cost is a major concern, some low-cost, low-bandwidth components are employed. The data to be modulated are generated offline as described in previous chapters. For the conversion to the analog domain an 8-bit Fujitsu DAC with a bandwidth of around $15 \mathrm{GHz}$ is employed. Because the PtP voltage at the output of the DAC (i.e. roughly $400 \mathrm{mV}$ ) is not enough to drive the modulator, an amplifier is needed. The MD is followed by a high bandwidth biasT. The signal is converted to optical domain by a $20 \mathrm{GHz}$ TOSA. Since the TOSA operates at $1300 \mathrm{~nm}$ the influence of CD is negligible. In [85] it is shown that, when using a similar TOSA, PAM-4 performance is almost the same for BTB, 10, 20, 30 and $40 \mathrm{~km}$ links. Thus, the focus of the experiments presented here is only on the BTB scenario, as the BTB results can be extrapolated to transmission distances of up to $40 \mathrm{~km}$. A VOA is used to control the received optical power. The optical signal is converted back into electrical domain by a $20 \mathrm{GHz}$ bandwidth ROSA and then data acquisition is performed by a real-time scope at 80 GSamples/s. The captured data are processed offline. First, they are resampled and then an AGC is used to adjust the signal power, enabling fast and valid channel acquisition in equalization blocks. Next, timing recovery is performed, followed by a FFE and MLSE. In the end, the Gray BER is calculated.

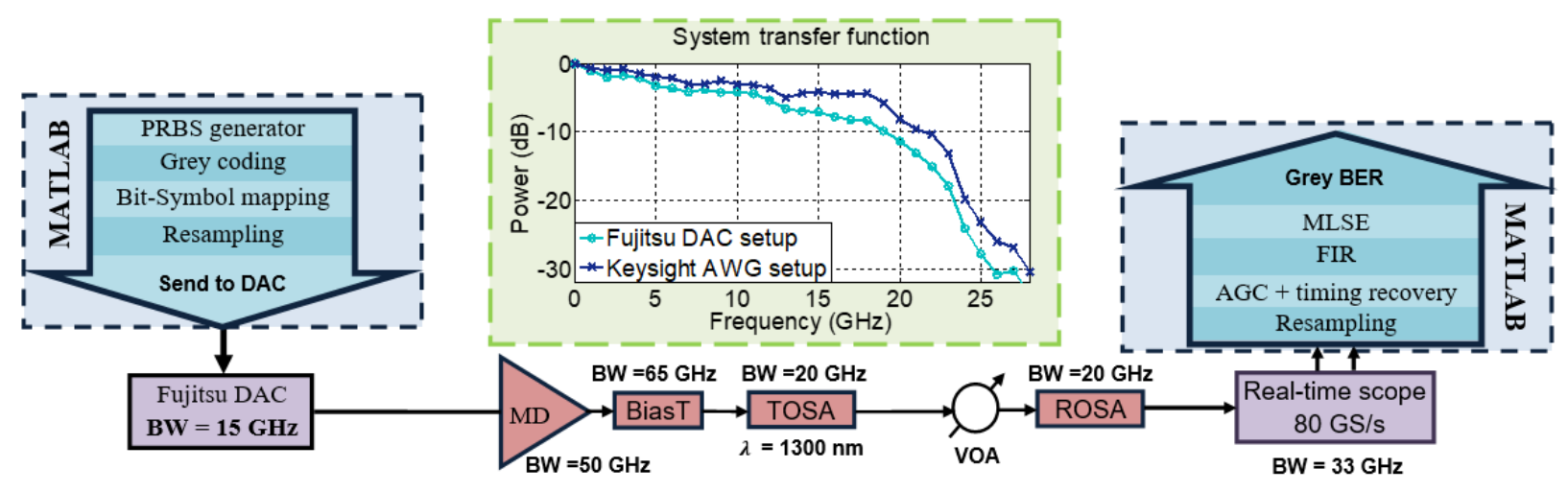

Figure 5.4. Experimental setup for testing 100 Gbps PAM transmissions 


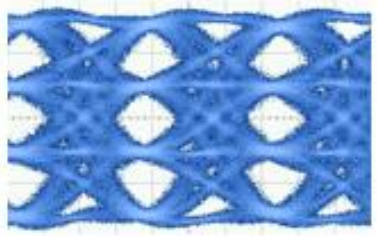

a)

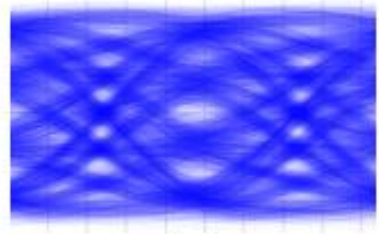

b)

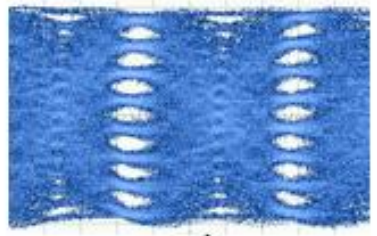

c)

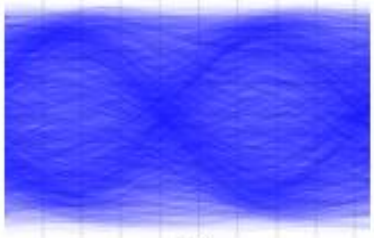

d)

Figure 5.5. 28 GBaud PAM-4 eye diagrams after DAC (a) and after ADC (b) and 19 GBaud PAM-8 eye diagrams after $D A C(c)$ and after $A D C(d)$

The overall bandwidth of the experimental setup, shown as inset in Figure 5.4, is severely limited. When using the DAC to generate the electrical signal the 3-dB cut-off frequency of the overall setup will be only $6 \mathrm{GHz}$. Furthermore, the spectral power starts dropping fast for frequencies higher than $18 \mathrm{GHz}$. The bandwidth limitations are reflected in the received eye diagrams, as can be observed from Figure 5.5. When calculating the BERs corresponding to the received PAM-4 eye diagram, it can be observed that the performance is very poor, where BERs are well above the FEC threshold (see Figure 5.6(a), the dotted black line). An 81-tap FFE followed by a 16-state MLSE are used in order to improve the BER. This combination of DSP algorithms ensures BERs below the HD-FEC limit of $4 \times 10-3$ at $-10.6 \mathrm{dBm}$ received power for a 56 Gbps PAM-4 signal and at $-5.5 \mathrm{dBm}$ for a $112 \mathrm{Gbps}$ PAM-4 signal, as shown in Figure 5.6(a). Because of the severe bandwidth limitations, at baud rates of 48 GBaud or higher (as demonstrated also in the previous section where a similar setup was used, see Figure 5.3) the received data is closer to a 7-level DB signal than it is to the transmitted PAM-4. As a result of this, the MMA used to train the tap values of the FFE is no longer able to properly converge. If the MMA algorithm is modified to converge to a 7-level signal, then the BER at $112 \mathrm{Gbps}$ can be lowered from $2 \times 10^{-4}$ down to $10^{-5}$ at a received power of $-2 \mathrm{dBm}$ and the sensitivity is improved from $-5.5 \mathrm{dBm}$ down to $-6.6 \mathrm{dBm}$, a more than 1 $\mathrm{dB}$ gain.
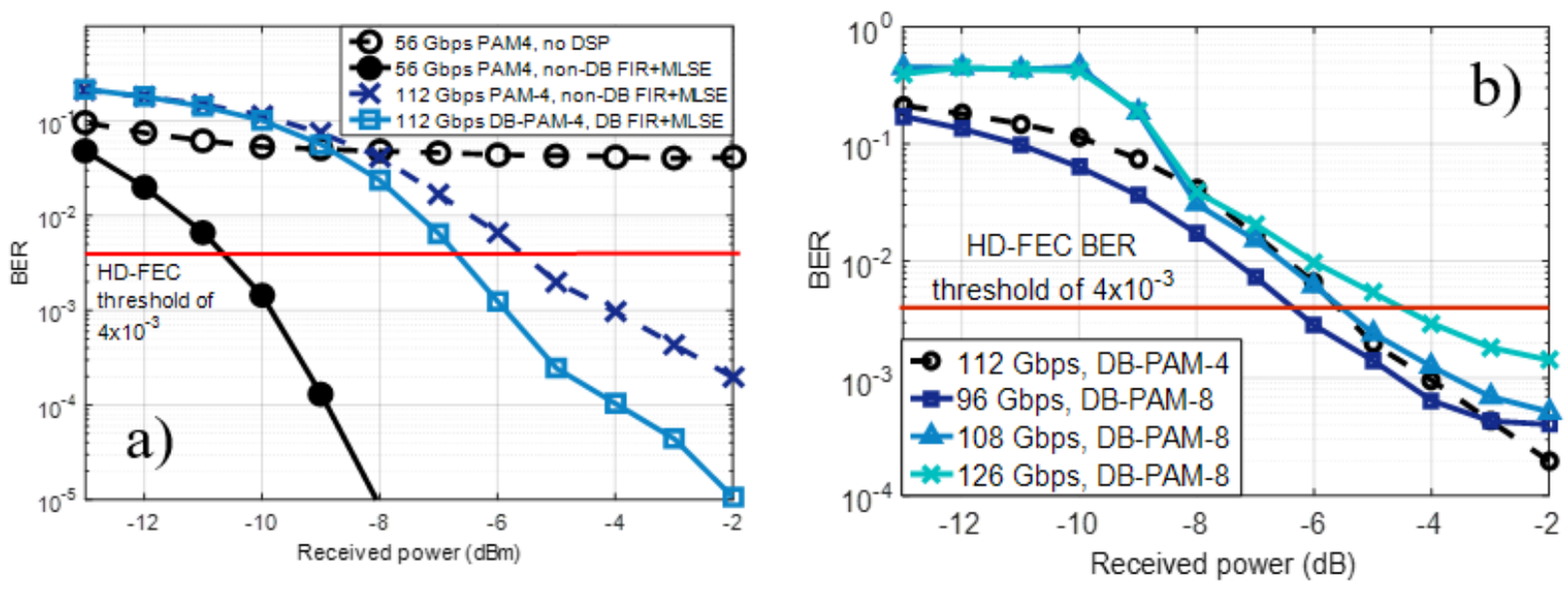

Figure 5.6. 56 and 112 PAM-4 sensitivity vs. BER curves (a) and PAM-4 vs. PAM-8 sensitivity performance comparison (b). DSP: 81-tap FFE followed by a 16-state (for PAM-4) or 64-state (for PAM-8) MLSE 
Due to the limited sampling rate of the DAC and real-time scope and because of the low bandwidth of the experimental setup, for data rates above $112 \mathrm{Gbps}$, BERs below $4 \times 10^{-3}$ are no longer achievable with PAM-4 or DB PAM-4 signaling. These limitations make the use of even higher order modulation formats attractive for 400G WDM networks. In order to test if further increasing the modulation order can enable higher bit rates, a PAM-8 experiment using the same setup as in Figure 5.4 is performed. The performance of PAM-8 in terms of BER was evaluated at 96, 108, and $126 \mathrm{Gbps}$ and the respective BER curves are shown in Figure 5.6(b). The PAM-8 scheme is more sensitive to the SNR than PAM-4 and as a result, at speeds of 108 Gbps, PAM-8 cannot achieve BERs lower than PAM-4 can at 112 Gbps. However, since it requires a lower baud rate than PAM-4, PAM-8 provides BERs below the HD-FEC threshold at data rates as high as 126 Gbps, as seen in Figure 5.6(b), improving upon the PAM-4 maximum bit rate of 112 Gbps. Increasing the bit rate beyond $126 \mathrm{Gbps}$ cannot be achieved with the proposed setup and at BERs below the FEC threshold.

It is demonstrated here that bit rates of $100 \mathrm{Gbps}$ and higher are possible with PAM-n schemes when using low-cost, 20G components. PAM-4 and DB PAM-4 are possible at speeds of up to 112 Gbps. We also prove that PAM-8 can enable higher data rates than PAM-4. Transmissions speeds of up to $126 \mathrm{Gbps}$ have been demonstrated using PAM-8. While it can enable faster transmissions, PAM- 8 has worse performance than PAM-4, when the two modulation schemes are compared at similar bit rates. Typically PAM-8 also exhibits a much higher error floor than PAM4. Thus, it can be concluded that for $100 \mathrm{G}$ single-lambda optical IM-DD transmission PAM-4 is the most suited amplitude modulation.

\subsection{Digital pre-emphasis}

In the case of short reach optical transmissions like metro-access networks or data-center interconnections, low cost and compact solutions are desirable. IM-DD systems are preferred in this scenario. The most widespread approach to IM-DD is to use the OOK modulation. While simple to implement and with a high tolerance to noise, it is also very sensitive to bandwidth limitations. Next generation short reach optical links are moving towards 400G WDM, with singlewavelength data rates of up to $100 \mathrm{~Gb} / \mathrm{s}$ [68]. Although OOK transmissions of up to $100 \mathrm{Gbps}$ have been demonstrated [64], they require high-cost, high-bandwidth components. With bandwidth requirements becoming more severe, higher order modulation formats like PAM-4, DMT or CAP are being considered [86], [67]. Out of these options the PAM-4 scheme seems most likely to be adopted in 400G WDM networks [68]. As shown in the previous section PAM-8 can also be employed instead of PAM-4, in order to reduce the bandwidth requirements of the transmission. The drawback is an increased sensitivity to noise, which makes PAM-8 less desirable. The aim of this section is to investigate a different approach to the bandwidth limitations problem, namely digital pre-emphasis (instead of the approach of increasing the modulation order).

As is made clear in this chapter, very high baud rate optical IM-DD transmissions suffer from extreme bandwidth limitations. Typically, a FIR filter is used at the receiver side to equalize the 
spectrum of the received signal in non-coherent systems. However, this filter cannot perfectly reproduce the spectrum of the transmitted signal due to the presence of other impairments, such as noise and nonlinear effects. Another approach is to pre-compensate for the transfer function of the transmission system at the transmitter side, in the digital domain, before the data is loaded into the DAC. This technique is known as DPE.

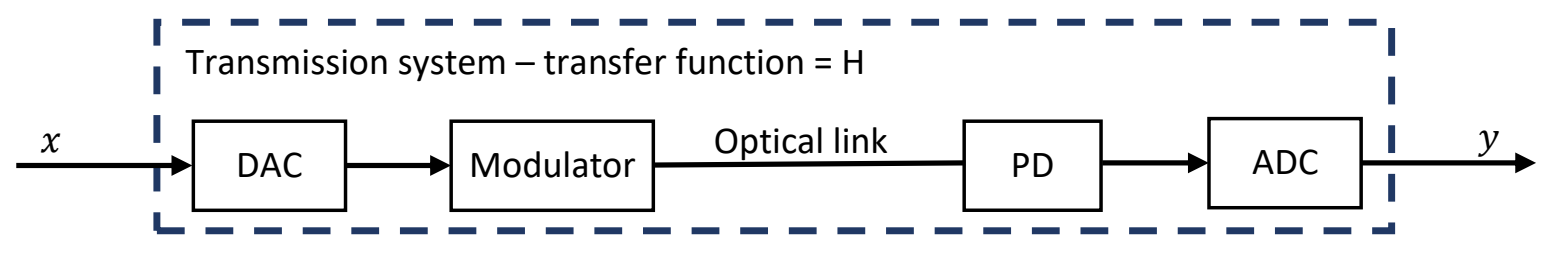

Figure 5.7. Block diagram of a basic optical transmission system

Let us consider the basic transmission system in Figure 5.7. In this block diagram $x$ is the transmitted data and its spectrum can be denoted with $X$, while $y$ is received data with the spectrum $Y$. The optical transmission system in this example introduces bandwidth restrictions on $X$ which can be referred to as the transfer function of the system and are denoted with $H$. The spectrum of the received signal can be expressed as:

$$
Y=H * X, \quad Y[d B]=X[d B]-H[d B]
$$

The ideal transfer function would be equal to $0 \mathrm{~dB}$ for all spectral components, meaning that $Y=$ $X$. Of course in reality that is never the case. In theory the optimum pre-emphasis function is the inverse of the system transfer function.

$$
H_{D P E}=H^{-1}, \quad H_{D P E}[d B]=-H[d B]
$$

If the pre-emphasis function is applied to the spectrum of the transmitted data then the channel will reverse the effects of the DPE and the received data would be equal to the original transmitted data (before DPE).

$$
X_{D P E}=X * H_{D P E} \rightarrow Y=X_{D P E} * H=X * H_{D P E} * H=X * H^{-1} * H=X
$$

In reality though this approach to DPE is faulty. In order to demonstrate the disadvantages of the method presented above a MATLAB simulation is implemented (which closely emulates the bandwidth of a real experimental setup), where a 56 GBaud PAM-4 signal is transmitted BTB through a bandlimited system. The spectrums $X, H, H^{-1}$ and the approximated $H^{-1}$ are shown in Figure 5.8. It can be seen in this figure that the spectral power at frequencies close to the Nyquist frequency is very low, dropping by more than $20 \mathrm{~dB}$. This means that at high frequencies the spectral power of the signal will be close or even below that of the noise. If we are to simply inverse the system transfer function the high frequencies power will be heavily boosted and, in doing so, the noise is also amplified, leading to a lower SNR. 


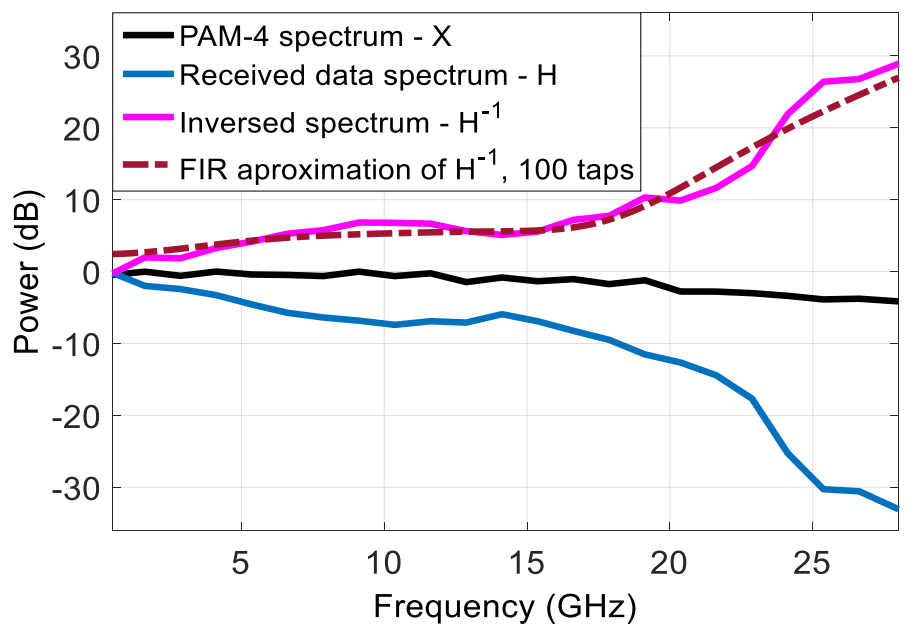

Figure 5.8. The spectrums for a 112 Gbps PAM-4 employing DPE

Another detrimental effect of heavily boosting the spectral power at high frequencies is an increase in the peak-to-average power ratio (PAPR). As the name implies the PAPR is the ratio between the maximum power of the signal and the average power. A classic PAM-4 signal has constant PtP amplitude over one UI. By amplifying the high frequencies the amplitude in the middle of the eye remains more or less constant but the amplitude between the eyes is increased, resulting in an increase in PAPR. Since the amplitude range of the DAC remains constant no matter what preprocessing is done on the signal prior to loading it in the DAC, the higher PAPR translates into lower amplitude difference between modulation levels at the output of the DAC and thus lower SNR. The two phenomena presented above make it so that the performance when the inversed transfer function of the transmission system is employed as the DPE function is significantly worse than when no DPE is used.

A different approach is needed to the pre-emphasis problem. Obviously, the high frequencies must not be fully compensated. Another issue not discussed in the previous paragraph is the high number of taps required to closely approximate $H^{-1}$ (see approximation with 100 taps in Figure 5.8, dotted line). An experiment is prepared in order to investigate a different approach to DPE in bandlimited systems and faster than Nyquist transmissions. Let us first have a look at the experimental setup, which is shown in Figure 5.9. Using MATLAB, a pseudo-random sequence of PAM-4 symbols is generated. The PAM-4 data are pre-distorted by a DPE filter, then resampled, and finally loaded into the DAC. An 8-bit Fujitsu DAC is employed, with a 3-dB bandwidth of roughly $15 \mathrm{GHz}$. The output of the DAC is amplified by a MD and passed through a biasT circuit, before being modulated by a $20 \mathrm{GHz}$ bandwidth TOSA. Since the TOSA is operating at $1300 \mathrm{~nm}$ wavelength, the effects of $\mathrm{CD}$ are negligible. For this reason these experiments are focused on the BTB scenario. A VOA is used to adjust the optical power. The signal is converted back into the electrical domain by a ROSA, with a bandwidth of roughly $20 \mathrm{GHz}$, and then data acquisition is performed by a real-time oscilloscope at 80 GSamples/s. The offline data are up-sampled to two samples/symbol and then timing recovery is performed. Because of the severe bandwidth 
limitations a FFE followed by MLSE are employed. The performance of the transmission is evaluated by calculating the Gray BER.

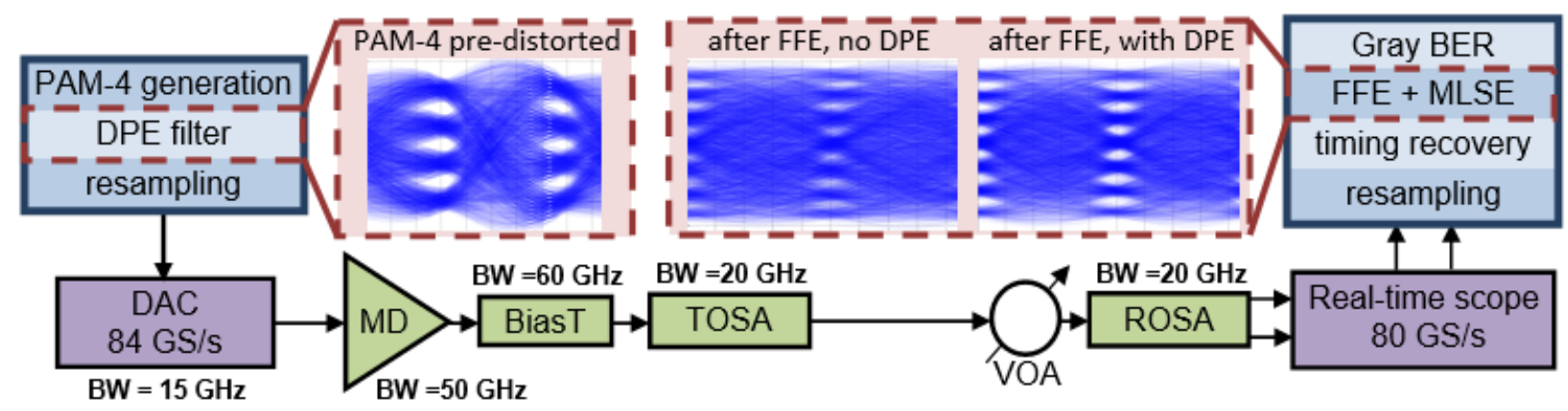

Figure 5.9. Experimental setup for testing DPE techniques at high baud rates

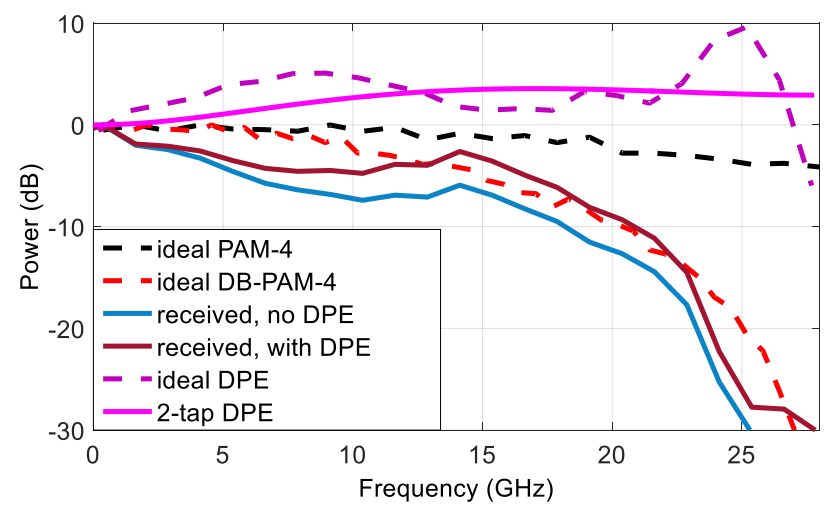

Figure 5.10. Spectrums related to the 2-tap DPE

In order to design a pre-emphasis function for this experimental setup, an analysis of its transfer function must be performed. In Figure 5.10 the spectrums of a PAM-4 and a DB PAM-4 signals are shown (dashed black line and dashed red line respectively), before being sent to the DAC. Ideally (i.e. no noise and perfect DPE) this should also be how their spectrums look after the ADC. In the same figure the spectral power of the PAM-4 signal after transmission is displayed with continuous blue line. It can be seen that, from a spectral point of view, the received signal spectrum, $Y$, is much closer to a DB spectrum than it is to the classic PAM-4 one. In chapters 5.2 and 5.3, where similar experimental setups are employed, the performance is better when assuming a DB signal after ADC. Thus, it makes perfect sense that the DPE should target to reproduce a DB PAM-4 signal at the receiver side. The target spectrum $Y_{D P E}$ becomes the DB PAM-4 spectrum in this case. The target pre-emphasis function $H_{D P E}$ can be expressed as:

$$
H_{D P E}=Y_{D P E}-Y
$$

and can be seen in Figure 5.10 with a purple dashed line. Now the $H_{D P E}$ no longer requires emphasis of more than $10 \mathrm{~dB}$ at high frequencies (as would have been the case if the classic PAM4 spectrum would have been targeted, see Figure 5.8). Since complex DSP is already used in this scenario (91-tap FFE + 16-tap MLSE), it is required that the complexity of the DPE is very low. 
That is why the number of FIR taps employed to approximate the target pre-emphasis function was limited to 2. The MATLAB function 'fir2' is used here to replicate as closely as possible the $H_{D P E}$ (with only a 2-tap FIR) and the resulting function is shown in Figure 5.10 with a continuous pink line. The equivalent DPE filter in time domain is $1-0.15 z^{-1}-0.1 z^{-2}$. When this DPE filter is added, the spectrum of the received signal will approach the one of the DB PAM-4, as seen with dark red continuous line in Figure 5.10.
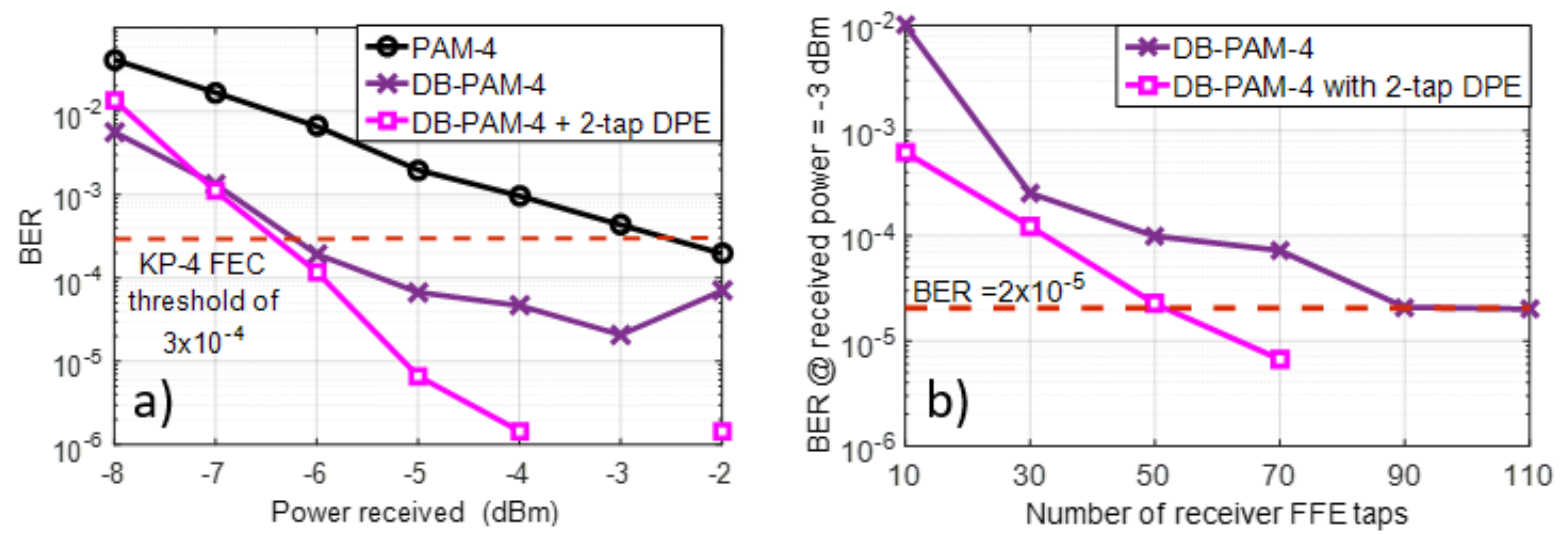

Figure 5.11. Sensitivity performance comparison between PAM-4, DB PAM-4 and DB PAM-4 with a 2tap DPE filter (a) and FFE complexity vs. performance curves for DB PAM-4 with and without DPE (b). All results are for a $112 \mathrm{Gbps}$, BTB transmission

In order to test the assumptions made in the previous paragraph a PAM-4 signal is transmitted BTB using the experimental setup in Figure 5.9. At the receiver side the equalizer is trained to detect first a regular PAM-4 signal and then a DB 7-level signal. The sensitivity curves of the PAM-4 and DB PAM-4 transmissions are shown in Figure 5.11(a). It can be seen in this figure that when employing DB signaling the BER is lowered by about one decade, regardless of the received power. While PAM-4 BERs can barely go below the KP4 FEC threshold of $3 \times 10^{-4}$, DB PAM-4 achieves BERs as low as $2 \times 10^{-5}$.

To further reduce the BER, DPE is employed. A 2-tap filter is considered for the pre-emphasis filter at the transmitter side. The desired pre-emphasis transfer function and its approximation by a 2-tap filter can be seen in Figure 5.10. By applying the DPE filter before loading the data into the DAC, the spectrum of the received data is brought closer to that of DB PAM-4. This translates into an improved eye diagram after the FFE, as shown in the inset to Figure 5.9, and into BERs 10 or more times lower than in the case of a transmission without pre-distortion, as illustrated in Figure 5.11(a). At a received power of $-3 \mathrm{dBm}$ the BER floor is removed (i.e. $B E R=0$ ) when preemphasis is used. However, due to the limited number of symbols processed $\left(3 \times 10^{21}\right)$, a BER of 0 should be interpreted as being lower than $10^{-6}$. The gain in terms of BER can be traded for reducing the complexity of the FFE. From Figure 5.11(b) we see that the minimum achievable BER without using pre-emphasis is $2 \times 10^{-4}$ and that at least 90 FFE taps are needed, while to accomplish the same thing when DPE is enabled only a 50-tap FFE is required. In general, the 2-tap DPE reduces the required number of taps at the receiver side by roughly $40 \%$. 


\subsection{Partial response signaling for CD mitigation}

The advent of new technologies like $5 \mathrm{G}$ and $4 \mathrm{~K}$ resolution is increasing the demands on shortreach metro-access networks. For transmission distances lower than $100 \mathrm{~km}$ coherent and noncoherent solutions are competing for market share. While coherent technologies provide better performance and higher data rates, they require more expensive components and complex DSP at the receiver side. That is why IM-DD implementations are usually preferred over coherent ones for next generation 100G/400G metro-access optical networks [68], [86], [87]. One of the main issues with IM-DD transmissions is that they cannot cover more than a few tens of kilometers at data rates of 28 Gbps or higher [23], [88] without the use of a DCM, DCF or CD pre-compensation. All of these solutions are imperfect and there is always some residual CD leftover after transmission. Employing a higher order modulation like PAM-8 can improve the residual CD tolerance by lowering the baud rate [89], however the increased SNR/OSNR requirements make such modulation unfeasible for most metro-access network scenarios. Another approach to handling the $\mathrm{CD}$ problem is to transmit the signal in the O-band, instead of the C-band. In the Oband, at wavelengths around $1310 \mathrm{~nm}$, the dispersive effects in the fiber are negligible. The downsides of this solution are that the optical fiber attenuation coefficient is higher in the O-band than in the C-band and that EDFAs operate in the $\mathrm{C}$ and L-bands. These problems limit the maximum range of an optical transmission at $1310 \mathrm{~nm}$ to around $40 \mathrm{~km}$ at bit rates of $56 \mathrm{Gbps}$ [85], [4].

Transmitting an optical signal over a SSMF will introduce phase distortion which scales squarely with frequency [90], [91]. This means that higher frequencies will be affected more by CD than lower frequencies. This, in turn, implies that the less bandwidth a signal's spectrum occupies the less said signal is affected by dispersive effects. In order to reduce the spectrum occupancy at higher frequencies without changing the baud rate it is necessary to introduce some controlled ISI. This technique is widely referred to as PRS. While PRS is normally employed in order to increase the spectral efficiency of a signal (see chapter 5.1), in this section it is presented as a way of increasing the $\mathrm{CD}$ tolerance of an optical transmission in the C-band. The advantages and disadvantages of this approach are also discussed here. As far as we know this is the first time PRS is optimized for improving CD tolerance in a short-reach IM-DD optical transmission and the only other similar approaches are the narrowly filtered on-off-keying (NF-OOK) modulation format [92] and the so-called combined amplitude-phase shift (CAPS) coding scheme [93], [94].

As made evident by the previous sections of Chapter 5, the most widespread version of PRS employed in today's non-coherent optical systems is the DB signaling, which is employed as a means of improving the performance of heavily bandlimited optical systems [15], [82]. Since the spectrum of a DB signal after transmitter is (approximately) half of that of the original signal, as shown in Fig. 1(a), it follows that this form of PRS should also help increase the CD tolerance of a transmission. In order to prove that this is the case a simulation was performed at $28 \mathrm{Gbps}$, emulating a $10 \mathrm{GHz}$ and a $20 \mathrm{GHz}$ bandwidth MZM transmitter and PIN receiver. No DSP algorithms were employed. The results of this simulation can be observed in Figure 5.12(b). In the 
BTB case with $20 \mathrm{GHz}$ components, OOK and DB OOK have approximately the same performance. However, when the bandwidth is limited at $10 \mathrm{GHz}$, the DB version will outperform classic OOK by roughly $2 \mathrm{~dB}$ in terms of OSNR. When looking at the CD tolerance of the two modulation schemes it can be seen that while OOK can handle a transmission over up to $14.7 \mathrm{~km}$ of fiber, DB can handle up to $22.4 \mathrm{~km}$, for OSNR values below $24 \mathrm{~dB}$. For bandlimited systems (i.e. the $10 \mathrm{G}$ bandwidth case) DB can help improve transmission distance by roughly 1.8 times, while for higher bandwidth systems (i.e. the 20G bandwidth case) the gain is only around 1.4 times. In the next paragraphs a new type of PRS, customized for increased CD tolerance, is introduced. To differentiate it from regular DB (which is also a form of PRS) we will refer to this customized PRS simply as PRS (while classic duobinary is abbreviated with DB).
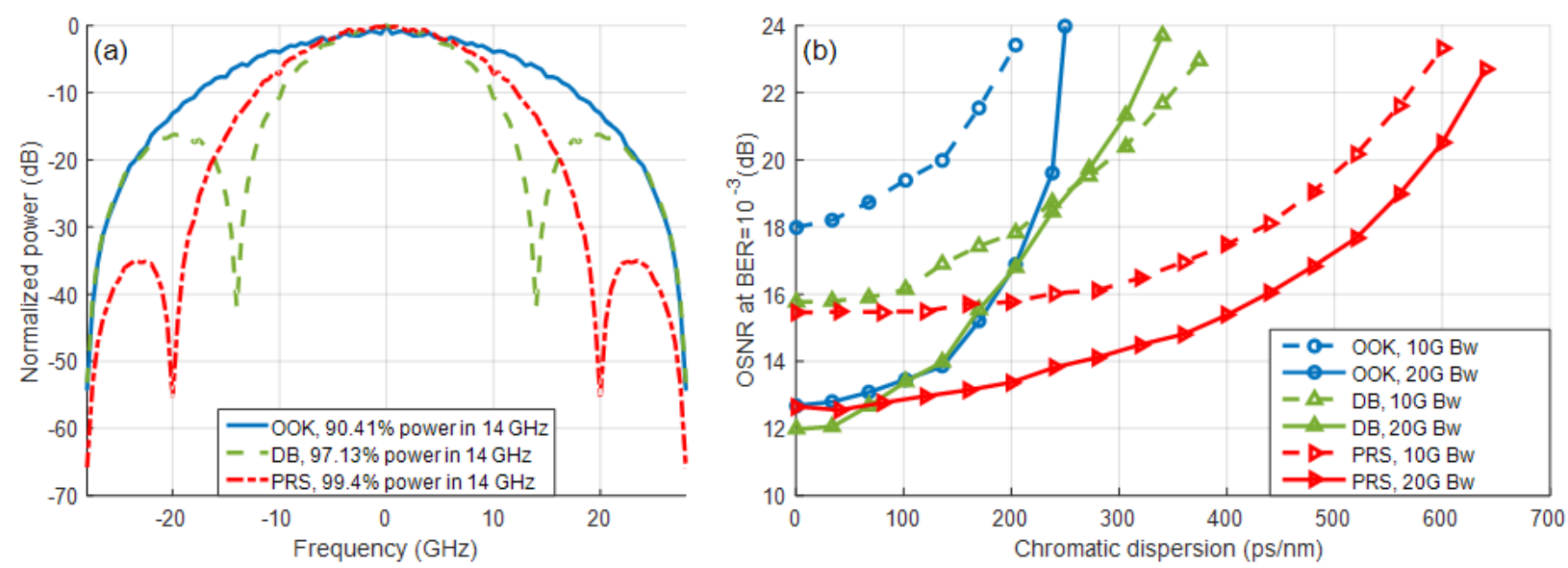

Figure 5.12. Spectrum comparison (for a 28 Gbps transmission) between $O O K, D B$ and $P R S(a)$ and $C D$ tolerance comparison (for the same 28 Gbps transmission) between $O O K, D B$ and PRS in low (10 GHz bandwidth) and high bandwidth (20 GHz bandwidth) scenarios (b)

From Figure 5.12(a) a correlation between the spectral power concentration of a signal and the CD tolerance of the same signal can be deduced. A 28 Gbps OOK signal has $90.41 \%$ of its spectral power concentrated at low frequencies (i.e. in a $14 \mathrm{GHz}$ bandwidth). Meanwhile its DB counterpart has $97.13 \%$ of its power concentrated in the -14 to $+14 \mathrm{GHz}$ frequency range. It follows that if a signal has even more spectral power concentrated at low frequencies then it should also be more efficient at handling CD. In order to maximize the spectral power at low frequencies the transmitted pulse should approximate the prolate spheroidal wave shape [95], [96], which is very similar to the Gaussian waveform shape. As a result, the DB pulse is adjusted to resemble more the Gaussian pulse (Figure 5.13, bottom left), thus obtaining a more CD tolerant form of PRS. In Figure 5.13, a comparison between the OOK, DB and improved PRS pulses and eye diagrams is shown. For simplicity and a fair comparison between DB and PRS the introduced ISI of the PRS is limited to just one symbol period. 

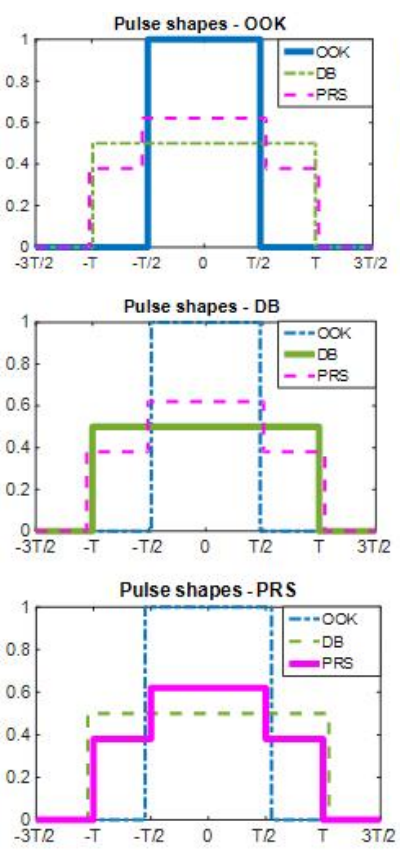

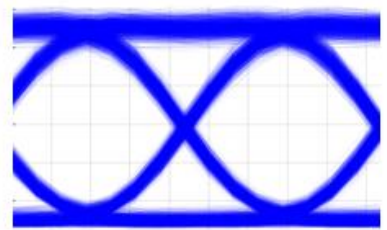

BTB eye diagrams

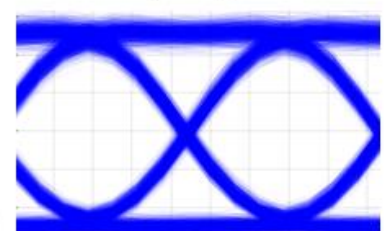

BTB eye diagrams

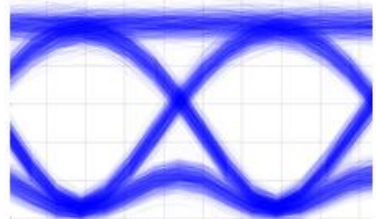

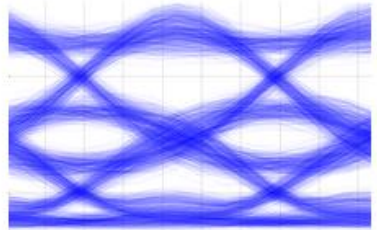

Eye diagrams after $15 \mathrm{~km}$

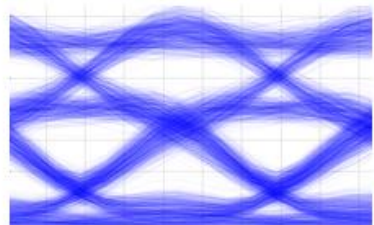

Eye diagrams after $15 \mathrm{~km}$

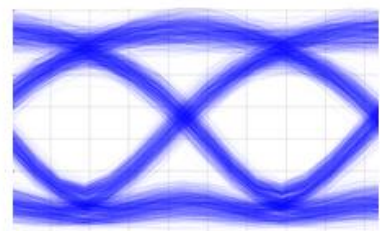

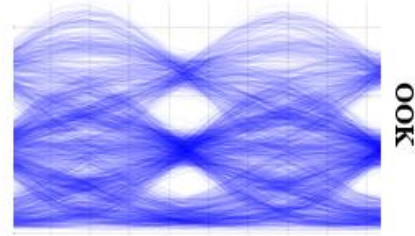

Eye diagrams after $30 \mathrm{~km}$

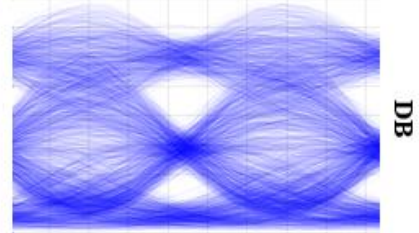

Eye diagrams after $30 \mathrm{~km}$

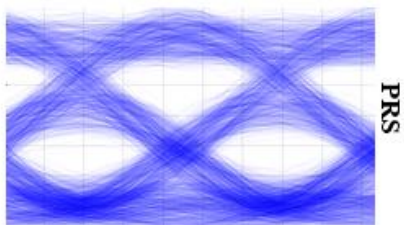

Figure 5.13. Pulse shapes (first column) for OOK (first row), DB (second row), and PRS (third row) and the corresponding eye diagrams for BTB (second column), $15 \mathrm{~km}$ (third column), and $30 \mathrm{~km}$ (fourth column). The eyes are obtained employing 20G components and a MZM, OSNR $=25 \mathrm{~dB}$

The performance of the PRS was tested in the same simulation scenario described in previous paragraphs and the results are displayed in Figure 5.12, together with those for OOK and DB OOK. It can be seen there that the spectrum of the PRS is even more concentrated than that of the DB at low frequencies, having $99.4 \%$ of its power within the -14 to $14 \mathrm{GHz}$ bandwidth (Figure 5.12(a)). This also translates into a significant increase in reach. When using PRS, link lengths of up to 37.6 $\mathrm{km}$ can be achieved in simulation, which is almost double of what is possible when using DB and approximately 2.5 times better than OOK (Figure 5.12(b)).

The PRS scheme can be extended to higher order intensity modulation formats, such as PAM-4. However, when employing the PRS with this kind of modulation schemes, there is an additional issue that has to be considered. So far the results shown in this section were obtained with a MZM. When using OOK in an IM-DD scenario only half of the modulation range of the MZM can be employed (either only the positive or only the negative region of the transfer curve - see Figure 2.11). On the other hand DB and PRS can use the whole range since their lowest and highest levels encode the same symbol, as illustrated in Figure 5.14. That is why there is no OSNR penalty for the 3-level DB and PRS when compared with the 2-level OOK, as seen in Figure 5.12(b) (the continuous line curves). In the case of DB PAM-4 however, the highest and lowest levels do not encode the same symbol anymore and thus the modulation is again restricted to just half the range of the modulator. For example, if we assume a MZM range from -3 to 3, then in the case of DB OOK both -3 and 3 will encode symbol 1, while in the case of DB PAM-4 -3 encodes PAM-4 symbol 0 and 3 encodes PAM-4 symbol 2 (the PAM-4 symbols being 0, 1, 2 and 3). 


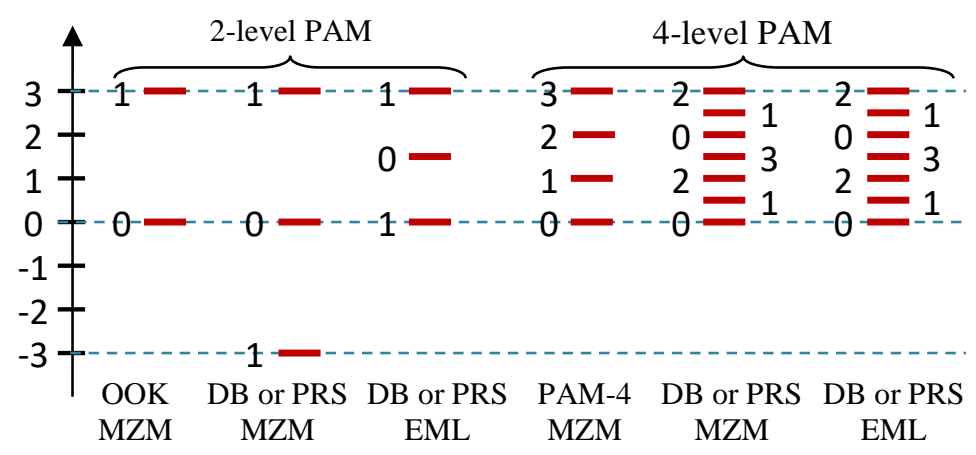

Figure 5.14. Symbol mapping for different modulation/modulator combinations

Another simulation is performed, this time at a bit rate of $50 \mathrm{Gbps}$ (a different bit rate than in the previous simulation is chosen to show that the relative gains provided by PRS are not influenced by the transmission speed), in order to evaluate the capabilities of PRS PAM-4. The results of this simulation are displayed in Figure 5.15. It can be observed in this plot that pure PAM-4 has more or less the same performance as PRS OOK in terms of CD tolerance but comes with a 4-5 dB OSNR penalty. PRS can help improve transmission length also when equalization is employed. To prove this a 16-state MLSE equalizer is implemented. For regular OOK the addition of the 16state MLSE more than doubles the link length, from 5 to $12 \mathrm{~km}$. The same holds true for PRS OOK, where adding MLSE extends reach from 11 to $22 \mathrm{~km}$. In the case of PAM-4 the addition of PRS increases the transmission distance by about 2.6 times (from 11 to $29 \mathrm{~km}$ ), having the downside of a $3 \mathrm{~dB}$ OSNR penalty over regular PAM-4 (as is to be expected when taken in consideration the explanation in the previous paragraph and Figure 5.14). Furthermore, by employing the 16-state MLSE the PRS reach can be almost doubled, from 29 to $52 \mathrm{~km}$. When comparing PRS OOK with PRS PAM-4 it can be observed that PAM-4 extends the link by approximately 2.4 times, with MLSE (from 22 to $52 \mathrm{~km}$ ) or without (from 12 to $29 \mathrm{~km}$ ). The high OSNR requirements of PRS PAM-4 and the limited linearity of commercially available electrical components make it difficult for this modulation scheme to be implemented experimentally and thus the experiments performed for this thesis are focused on PRS OOK (see next paragraphs).

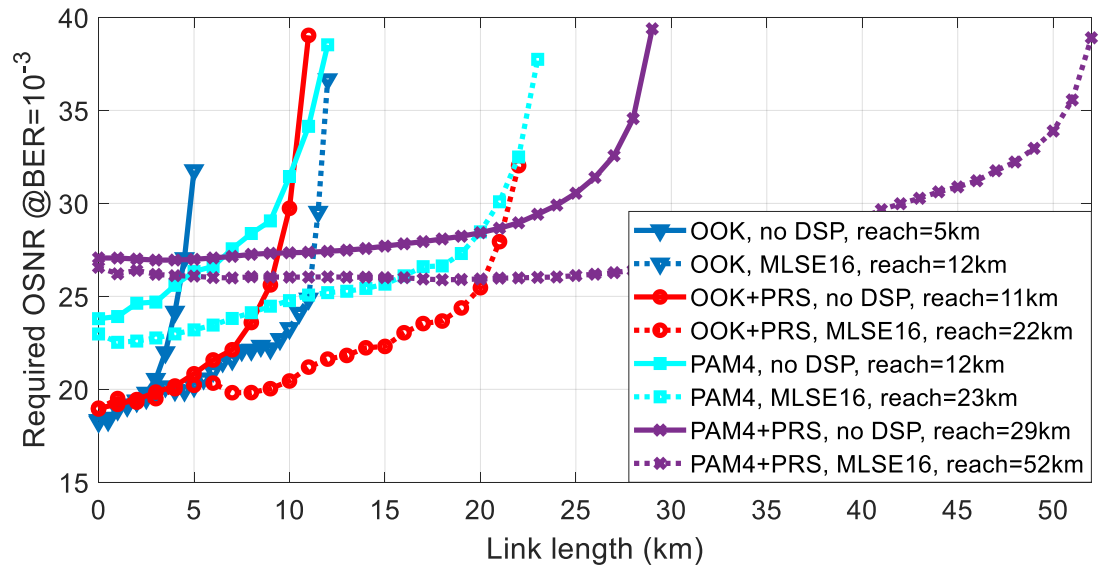

Figure 5.15. OOK/PAM-4 versus PRS OOK/PAM-4 BER curves 
In order to validate the simulation results an experimental setup was built, as shown in Figure 5.16. The first step is to generate a PRBS of length $2^{15}$ and then create the OOK or PRS signal from this PRBS. Next, a pulse shaping filter is applied (i.e. raised cosine). The data is then loaded into a 14 $\mathrm{GHz}$ bandwidth 84 GSamples/sec DAC which converts it to the analog domain. The signal is amplified and sent to the modulator. To take full advantage of the PRS properties a $25 \mathrm{GHz}$ bandwidth MZM is employed, however other modulators like the EML are valid options (although they come with an OSNR penalty cost for PRS, as described in previous paragraphs). After the signal is converted into the optical domain, ASE noise is added in order to control the OSNR. After 0 to $60 \mathrm{~km}$ of fiber an EDFA is employed, followed by an OBF which filters out the out-of-band noise. A $25 \mathrm{GHz}$ bandwidth PD is used to detect the signal and then an 80 GSamples/sec real-time oscilloscope captures the data. Next, timing recovery is performed, followed by either no receiver DSP whatsoever or by a 16-state MLSE. Finally, the Gray BER is calculated.

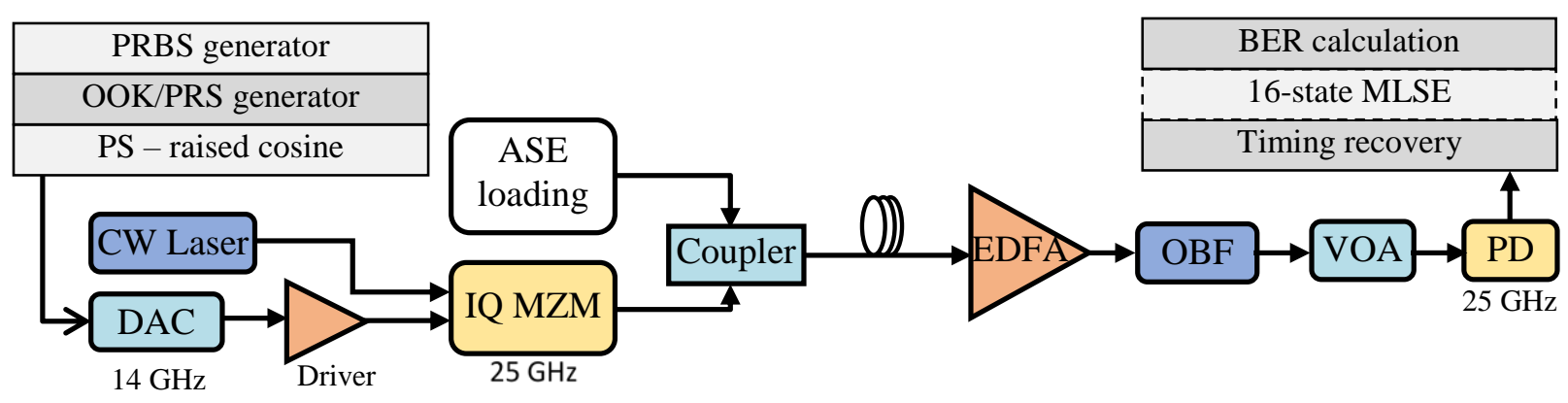

Figure 5.16. Experimental setup block diagram

The experiments were performed with link lengths of 0 (BTB), 10, 15, 20 and $25 \mathrm{~km}$ for OOK and 0 (BTB), 10, 20, 30, 40, 50, 60 and $70 \mathrm{~km}$ for PRS. The results of these experiments are displayed in Figure 5.17. In Figure 5.17(a) the required OSNR for a BER of $10^{-3}$ at different link lengths is plotted. When looking at the BTB performance it can be seen that PRS provides around $1 \mathrm{~dB}$ of OSNR gain. This is due to the fact that the experimental setup is bandwidth limited and that PRS, just like DB, is more resilient to bandwidth limitation than regular OOK. The OOK modulation is successfully transmitted over a maximum of $10 \mathrm{~km}$ of fiber at OSNR values below $20 \mathrm{~dB}$ and without the use of receiver equalization. If a 16-state MLSE is added then the transmission distance is increased to $20 \mathrm{~km}$. When PRS is employed the transmission range is extended to $30 \mathrm{~km}$ without equalization and to $60 \mathrm{~km}$ with a 16-state MLSE equalizer. If the reach of OOK and PRS are directly compared it can be observed that PRS triples the transmission distance, both with or without MLSE. The minimum length fiber spool available for this experiment was of $5 \mathrm{~km}$, thus the real link lengths might be a few kilometers longer (but less than $5 \mathrm{~km}$ ) than those presented in Figure 5.17(a).

The BER versus OSNR curves for the BTB case and for the longest achieved reach for OOK and PRS are plotted in Figure 5.17(b). While the longest reach is achieved for OSNR values below 20 $\mathrm{dB}$, in order to achieve very low BERs, below $10^{-5}$, an OSNR of up to $25 \mathrm{~dB}$ is required. It can be observed that (both with and without MLSE) when going down to lower BERs $\left(\right.$ BER $<10^{-3}$ ) OOK 
scales better than PRS. While having very low complexity, PRS (without extra DSP) outperforms OOK with 16-state MLSE and thus can be used to decrease complexity of non-coherent optical system in the C-band. It can be concluded from this experiment that, both when not using any receiver DSP and when using a 16-state MLSE, PRS can improve CD tolerance by almost three times in comparison with regular OOK.
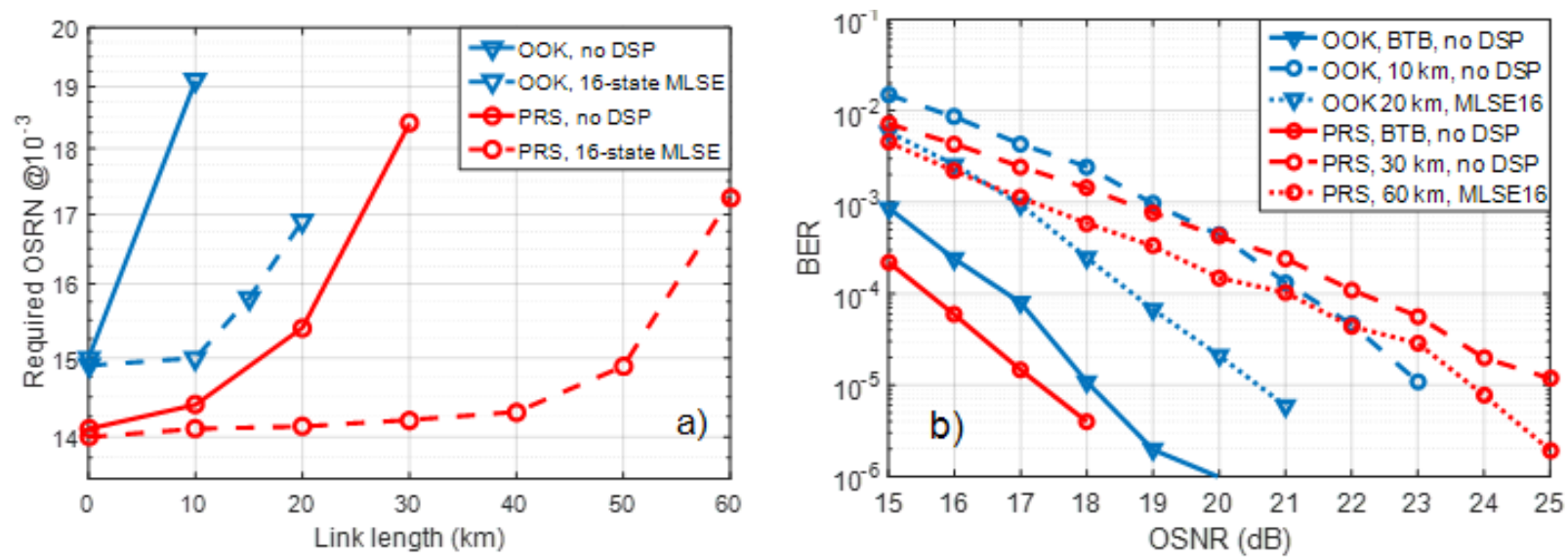

Figure 5.17. OOK versus PRS transmission length (a) and OSNR sensitivity curves for OOK and PRS for $B T B$ and maximum transmission length $(b)$

In this section it is shown that PRS can be employed specifically to combat the effects of CD. The DB signaling, one of the most widespread forms of PRS, requires less bandwidth than traditional non-DB modulation and it also improves transmission distances by approximately 1.4 to 1.8 times when compared with regular OOK. A more advanced form of PRS derived from DB is proposed and it is demonstrated here that it can increase the CD tolerance of a system by up to three times. When using MZMs and the OOK modulation there is no OSNR penalty when employing PRS, however, with other types of intensity modulators, an OSNR loss of around 4 to $5 \mathrm{~dB}$ for BTB can be observed. The PRS scheme presented here can be extended to higher order intensity modulation formats like PAM-4. When doing this the full range of the MZM can no longer be employed and thus we will see a 4-5 dB OSNR penalty regardless of modulator. 


\section{Chapter 6}

\section{Trellis coded modulation}

The current 10, 40, and 100 Gbps WDM solutions employed in short-reach optical networks are not enough to keep up with the ever-growing demand for internet traffic [77]. Recent standardization efforts are focused on delivering a $400 \mathrm{Gbps}$ alternative for short-reach optical communications [97]. Since cost is a major concern, IM-DD solutions are preferred over coherent ones. The OOK modulation format is easy to implement and has high tolerance to noise, thus it is the preferred solution for $4 \lambda \times 10$ and $4 \lambda \times 25$ Gbps WDM IM-DD systems. For the next generation $400 \mathrm{G}$ short-reach transmissions higher data rates are required, where $8 \lambda \times 50$ or $4 \lambda \times 100 \mathrm{Gbps}$ implementations are considered [97]. While OOK has been demonstrated at rates of up to around 100 Gbps [63], [64] it requires expensive high bandwidth components in order to achieve a low enough BER. For this reason research into 400G WDM systems is directed towards more advanced, higher order modulation schemes. The most likely candidates are PAM-4, CAP and DMT [12], [86], [98] . Equalization and FEC are required in order to achieve an error free (i.e. BER $<10^{-15}$ ) transmission at rates of $56 \mathrm{Gbps}$ or higher, when using the aforementioned modulation types. The most common equalizers employed in IM-DD optical systems are the FFE and the MLSE [10], as described also in chapter 3. For FEC, codes like Reed-Solomon (RS) and BoseChauduri-Hocquenghem (BCH) are considered [99].

In most modern optical systems modulation and FEC are treated as two separate processes, but they can also be integrated together. In this section we take a look at such a modulation format that incorporates FEC elements. This type of modulation is called TCM and was introduced by Ungerboeck in 1982 [100]. One of its more known uses is in the 1000BASE-T Gigabit Ethernet standard [101], [102]. To the best of the author's knowledge, TCM has not been investigated in the context of IM-DD 400G WDM optical networks. In this chapter a thorough investigation of TCM for data rates between 56 and 112 Gbps is provided. The main concepts behind TCM are introduced and the designing of such modulations is explained. After that two specific TCM schemes are introduced, the 4D PAM-4 TCM and 4D PAM-5 TCM. In order to provide results that are relevant for next generation $400 \mathrm{G}$ networks, the performance of these TCM solutions is evaluated against that of PAM-4, which is currently one of the most likely candidates for said networks [97]. The experiments are carried out using low-cost, commercially available components, at $1300 \mathrm{~nm}$ wavelength. 


\subsection{Construction of trellis coded modulation schemes}

The construction of the TCM is done at the transmitter side in the digital domain and it has two major components - the set partitioning and the convolutional encoding. These two parts are explained in detail in this chapter. Since convolutional codes are FEC, at the receiver side the direct recovery of the transmitted symbol is not possible, and a decoder is necessary (which in the case of TCM is a Viterbi algorithm-based decoder). In this section a generalized view of TCM is given, with more concrete examples of specific TCM schemes being presented in the following two subchapters.

The TCM is a modulation scheme that integrates elements of FEC in order to increase the noise tolerance. In terms of what can be observed in the channel, a TCM signal is basically a PAM- $n$ signal. To be able to fully benefit from this technique large constellations are required (typically larger than 32 points). Since a one-dimensional signal cannot encode such large number of points, a multidimensional approach is required. In coherent systems the signal has multiple dimensions (e.g. amplitude, phase, polarization) so one symbol could be enough in some scenarios to represent the full constellation. In IM-DD systems, however, only amplitude is used to encode information and in realistic high-speed transmission scenarios only up to 8-level amplitude modulations are feasible. Thus, more than one symbol needs to be grouped together in order to encompass the entire constellation, thus obtaining a multidimensional symbol, which will be referred to here as a supersymbol.

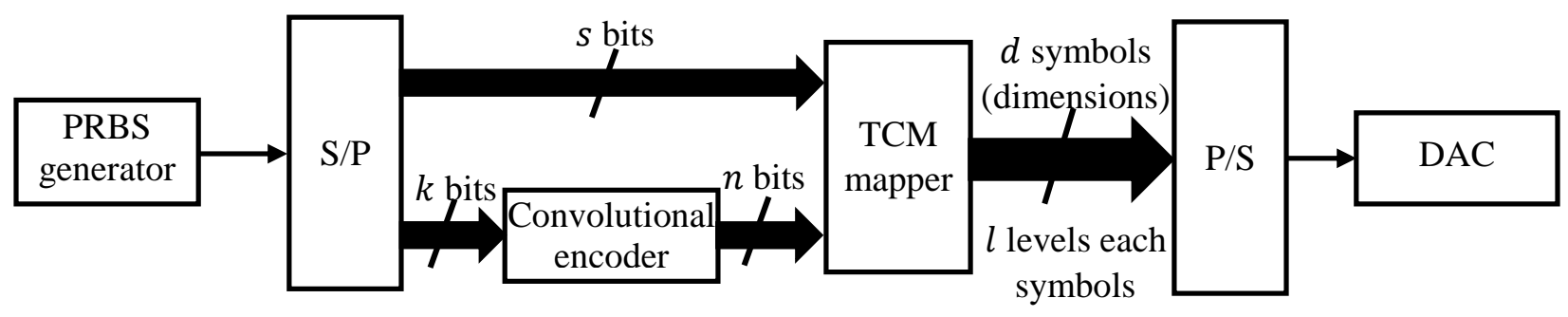

Figure 6.1. TCM generation block diagram

In Figure 6.1 an overview of the TCM generation is presented in the form of a block diagram. Before going into more details let us first define a set of parameters related to the whole generation process:

Table 4. List of parameters related to the generation of TCM

\begin{tabular}{|c|l|}
\hline Notation & Parameter explanation \\
\hline$p$ & Number of information bits for one super-symbol \\
\hline$t$ & Total number of bits mapped onto a single super-symbol \\
\hline$s$ & Number of bits that are being set partitioned \\
\hline$k$ & Number of encoded bits = number of bits at the input of the convolutional encoder \\
\hline
\end{tabular}




\begin{tabular}{|c|l|}
\hline$n$ & Number of bits at the output of the convolutional encoder \\
\hline$r$ & Number of redundancy bits added by the convolutional encoder \\
\hline$m$ & Number of memory registers of the convolutional encoder \\
\hline$d$ & $\begin{array}{l}\text { Number of symbols at the output of the TCM mapper = number of dimensions of the } \\
\text { TCM super-symbol }\end{array}$ \\
\hline$l$ & Number of levels of a single symbol \\
\hline
\end{tabular}

Now let us assume we want to transmit a stream of bits by employing the TCM modulation. A PRBS generator is used to create said bit stream. Next the stream is converted from serial to parallel, where the number of bits at the output of the converter, $p$, represents the number of information bits which are going to be mapped onto a single super-symbol. This number is split into $s$ bits which go directly to the mapper and $k$ bits which are sent to the convolutional encoder.

$$
p=s+k
$$

The convolutional encoder is a linear finite state shift register [19]. The $k$ input bits are shifted along linear shift register. The number of stages (i.e. the number of memory registers) of the encoder, $m$, determines the number states (the total number of possible combinations of values in the registers).

$$
N_{\text {states }, c c}=2^{m},
$$

where $N_{\text {states,cc }}$ is the number of states of the convolutional encoder. The convolutional encoder can be described by a group of generator polynomials ( $n$ polynomials in a group). These polynomials tell us how to obtain each output bit based on the input bits and the values of the memory registers. At the receiver side the convolutional code is decoded by employing the Viterbi algorithm. In order to get the most out of TCM the input to this Viterbi decoder must be soft information. The complexity of the decoder is high and scales exponentially with the number of memory registers, $m$ (complexity is similar to that of MLSE). Most TCM implementations operate with three or four memory registers. Since the classic Viterbi decoder outputs hard information other DSP algorithm that require soft input (like MLSE) cannot be used after the convolutional decoder. Since MLSE is also based on the Viterbi algorithm it also has a hard output, meaning that it can't be used before the convolution decoder either. There is a soft output version of the Viterbi algorithm (SOVA) which can be used together with TCM, however its performance is heavily affected by ISI, as is shown later in this chapter.

Since convolutional codes are a mature area they are not presented in more detail here as that will be outside the scope of this chapter, however more detailed descriptions of this FEC code can be found in [19], [103], [104]. The things that determine which convolutional code to use for a particular TCM are the number of input bits, $k$, the desired code rate and the complexity (which is determined by the number of states). 
The number of bits at the output of the convolutional encoder determines into how many subsets the TCM constellation is going to be partitioned in.

$$
N_{\text {subsets }}=2^{n}
$$

The size of the full TCM constellation is constrained by the total number of bits, $t$, at the input of the mapper as it has to map all these symbols:

$$
N_{c p} \geq 2^{t}
$$

where $N_{c p}$ is the number of TCM constellation points. The $N_{c p}$ is determined by the number of dimensions, $d$, and the number of levels of the chosen modulation, $l$ :

$$
N_{c p}=l^{d}
$$

To better illustrate the generation of a TCM scheme let us consider a more concrete example. Let us assume a PAM-8 transmission with a minimum distance $\left(d_{\min }\right)$ between the amplitude levels of 1 . We assume that the goal of TCM is to raise $d_{\text {min }}$ from 1 to 2 . When doing the set partitioning the $d_{\text {min }}$ between the points within any of the possible subsets is limited by the $N_{\text {subsets }}$. The relation between these two parameters can be expressed as follows:

$$
d_{\text {min,subset }} \leq \sqrt{\log _{2} N_{\text {subsets }}+1}=\sqrt{n+1}
$$

The equality is true when the set partitioning is done optimally. For the example presented here a minimum of eight subsets are required and, consequently, three bits at the output of the convolutional encoder. Each subset should have at least two points meaning that the full constellation should be at least 16 points large. The PAM-8 modulation provides an 8-point constellation; thus, it is not sufficient for our purpose. By grouping two consecutive PAM-8 symbols together we obtain a 2D PAM-8 super-symbol with a total of 64 constellation points. To take full advantage of this we need to assign eight points per subset (three bits), thus making sure all the constellation is used. Since $n=3$, the number of bits at the input of the convolutional encoder, $k$, can be either 1 or $2(k<n, k \neq 0)$. The bits encoding the subset number are more important because an error here automatically translates into errors when determining the point within the subset (we would be looking in the wrong subset) leading to error multiplication. Thus, it is a good idea to use a convolutional code with a $d_{\text {min }}$ larger than $d_{\text {min,subset }}$. A rate $2 / 3$ code $\left(k=2, n=3\right.$ ) with $m=3$ (eight states) has a $d_{\text {min }}=\sqrt{5}=2.24$ [19] and fits all of our requirements. Now we have all the elements required to define the TCM. Since the output super-symbol is made out of two PAM-8 symbols, the TCM presented here can be referred to as 2D PAM-8 TCM. In Figure 6.2 a block diagram of the 2D PAM-8 TCM is shown, where $b_{1}, b_{2}$ and $b_{3}$ are the three bits used to encode the point within a subset, $b_{4}$ and $b_{5}$ are the input to the convolutional encoder and $c_{1}$ is the redundancy bit generated by the convolutional encoder. Bits $b_{4}, b_{5}$ and $c_{1}$ are used to determine which subset is used. The output from the mapper is made out of the two PAM-8 symbols, $S_{1}$ and $S_{2}$. 


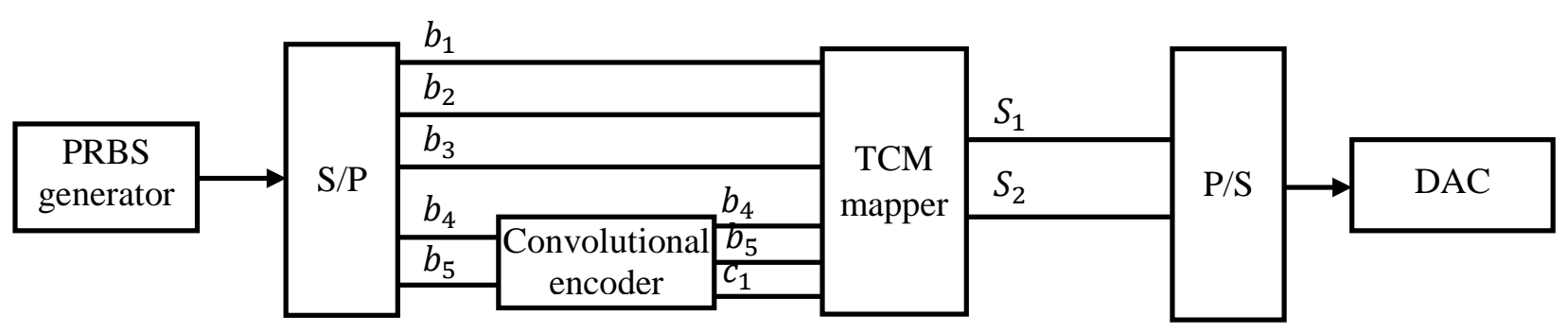

Figure 6.2. 2D PAM-8 TCM generation block diagram

In the optical channel TCM is indistinguishable from its parent modulation (e.g. for the 2D PAM8 TCM the parent modulation is PAM-8). After the TCM signal is converted back to the electrical domain at the receiver side a Viterbi algorithm decoder is used to determine the subset of each super-symbol. After the subset has been determined, the Euclidean distances between the received super-symbol and the subset points are calculated and the closest point is determined. Once the subset and the point within the subset are decoded the original transmitted bits can be recovered. The constellation size and memory of the convolutional code can be increased in order to improve the performance of TCM, however the complexity of the decoder grows exponentially with the memory and linearly with the constellation size.

Typically, TCM is employed to improve the noise tolerance of specific modulations. In the example presented above this modulation is PAM-8 (and the 2D PAM-8 TCM is the resulting TCM scheme). Thus, it makes sense that the results of TCM are compared with the parent modulation. For the particular example shown here, i.e. 2D PAM-8 TCM, five information bits are coded by two PAM- 8 symbols, resulting in a 2.5 bits/symbol transmission rate, while the PAM8 provides a 3 bits/symbol rate. This means that in order to achieve the same bit rate as PAM- 8 , 2D PAM-8 TCM has to transmit at a baud rate that is $6 / 5$ faster than that of the PAM-8. Another option would be to increase the number of levels of the output modulation and consequentially increase the constellation size, such that six information bits can be mapped and we wouldn't have to increase the baud rate (but the noise tolerance would be lowered). For the example presented in this chapter the number of points per subset would be increased from 8 to 16, resulting in a 128point constellation. The number of levels of the modulation at the output of the mapper can be increased to 12 in order to accommodate the new constellation size, resulting in a 2D PAM-12 TCM scheme that operates at the same baud rate as the original PAM-8 modulation.

\subsection{The 4D PAM-4 TCM scheme}

Advanced modulation formats enabled by DSP have been investigated for $400-\mathrm{G}$ datacenters applications in [105]. The mature OOK modulation format has been experimentally demonstrated for applications at $100 \mathrm{Gbit} / \mathrm{s}$ per wavelength rates [63], however such a solution would require very expensive high-bandwidth optics and electronics. The most promising modulation formats based on low-bandwidth, commercially available components are DB PAM-4 [15] and DMT [106]. Due to the DSP complexity, reliability and cost, a 28 GBaud rate is preferable [68]. In this 
chapter the performance of 56 Gbps PAM-4 and TCM transmissions is evaluated and compared, in the context of datacenter applications employing off-the-shelf 20-GHz 3-dB bandwidth TOSA and ROSA devices. The trellis-coded scheme employed here is a 4D PAM-4 version of TCM. The goal is to experimentally prove that 4D PAM-4 TCM outperforms PAM-4 and thus it is a solid solution for next generation $400 \mathrm{G}(8 \lambda \times 50 \mathrm{Gbps}) \mathrm{WDM}$ datacenter networks.

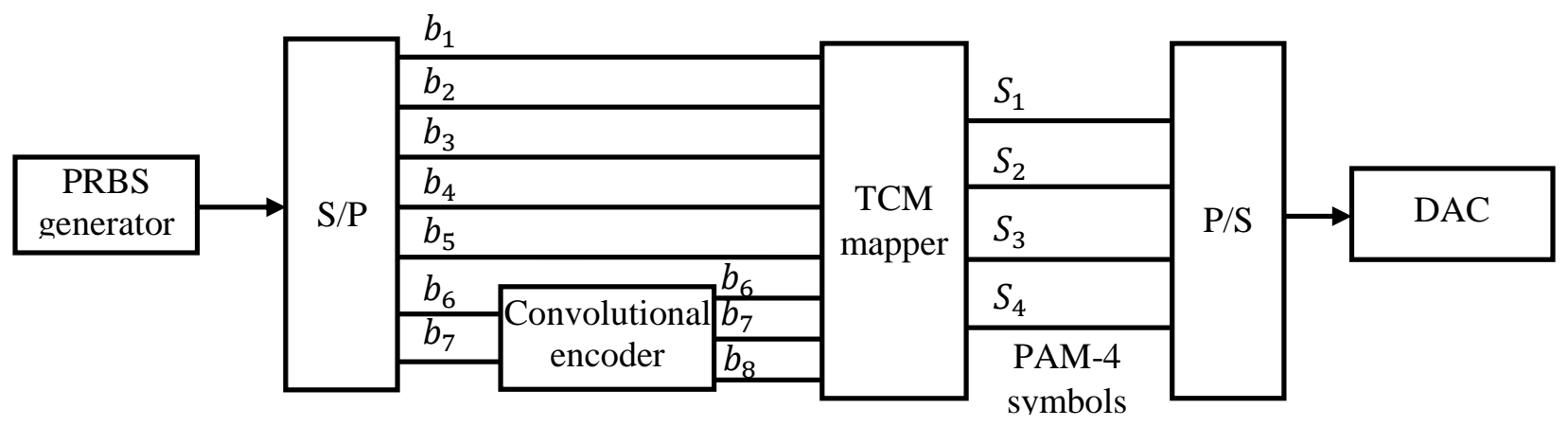

Figure 6.3. 4D PAM-4 TCM generation block diagram

As its name implies, the 4D PAM-4 TCM outputs four PAM-4 symbols at a time. Given the 4level modulation and equation (86), the number of points of the full constellation is equal to 256, meaning that a total of eight bits can be mapped onto this constellation. The generation block diagram of 4D PAM-4 TCM is displayed in Figure 6.3. The transmitted bit sequence is grouped in groups of 7 . The first five out of the seven bits are used to encode the point within a subset. The last two bits are sent to a convolutional encoder. An eight, redundancy bit is generated and together with the other two will encode the number of the subset. Having three bits for this purpose gives us a total of eight subsets. Each subset contains 32 points $(8$ subsets $\times 32$ points/subset $=256$-point constellation).

One aspect of TCM that was not fully explained in the previous section is how the data is partitioned into subsets. This process is introduced and explained in more detail in [100], [107]. From a theoretical point of view, as expressed also by equation (87), the maximum $d_{\text {min }}$ between the points within a subset (i.e. $d_{\text {min,subset }}$ ) is equal to the square root of $n+1$. In the case of 4D PAM-4 TCM the value of $n$ is 3 , resulting in a $d_{\text {min,subset }}$ of $\sqrt{4}=2$. Adapting the technique in [107] to the case presented here, the levels of the PAM-4 output $(0,1,2$ and 3$)$ are separated into two groups: $\mathrm{X}$ - even levels 0 and 2 and $\mathrm{Y}$ - odd levels 1 and 3. When working in 4D with four symbols at a time we can represent the TCM super-symbol in relation to the groups $\mathrm{X}$ and $\mathrm{Y}$. For example the super-symbol $\{1,0,2,3\}$ can be written as YXXY. With four groups for each supersymbol and two possible value in each group, a total of 16 points can be represented. Since the $d_{\text {min }}$ within a group (be it the even or the odd one) is equal to $2\left(d_{\min }=\sqrt{2^{2}+0^{2}}\right)$, the $d_{\min }$ within a four-group super-symbol is also at least 2. However, a 4D PAM-4 TCM subset has 32 points, thus a single four-group super-symbol is not enough to represent all points of a subset. Two four-group symbols are needed such that the $d_{\min }$ of this combination remains equal to 2 . To realize this we simply have to combine any four-group symbol with its inverse (e.g. YXXY with 
XYYX), thus ensuring a distance of at least 1 between each opposing groups $\left(d_{\min }=\right.$ $\sqrt{1^{2}+1^{2}+1^{2}+1^{2}}=\sqrt{4}=2$ ). The representation of each subset based on the odd/even groups is presented in the table below:

Table 5. 4D PAM-4 TCM subsets $\left(D_{\overline{1,8}}\right)$

\begin{tabular}{|c|c|l|}
\hline Subset & 4-group super-symbol representation & Number of points \\
\hline$D_{1}$ & $\mathrm{XXXX}+\mathrm{YYYY}$ & 32 \\
\hline$D_{2}$ & $\mathrm{XXXY}+\mathrm{YYYX}$ & 32 \\
\hline$D_{3}$ & $\mathrm{XXYX}+\mathrm{YYXY}$ & 32 \\
\hline$D_{4}$ & $\mathrm{XXYY}+\mathrm{YYXX}$ & 32 \\
\hline$D_{5}$ & $\mathrm{XYXX}+\mathrm{YXYY}$ & 32 \\
\hline$D_{6}$ & $\mathrm{XYXY}+\mathrm{YXYX}$ & 32 \\
\hline$D_{7}$ & $\mathrm{XYYX}+\mathrm{YXXY}$ & 32 \\
\hline$D_{8}$ & $\mathrm{XYYY}+\mathrm{YXXX}$ & 32 \\
\hline
\end{tabular}

In order to better illustrate the subsets and the constellation, a 3D PAM-4 TCM constellation is shown in Figure 6.4 (not 4D since only 3D can be represented in space).

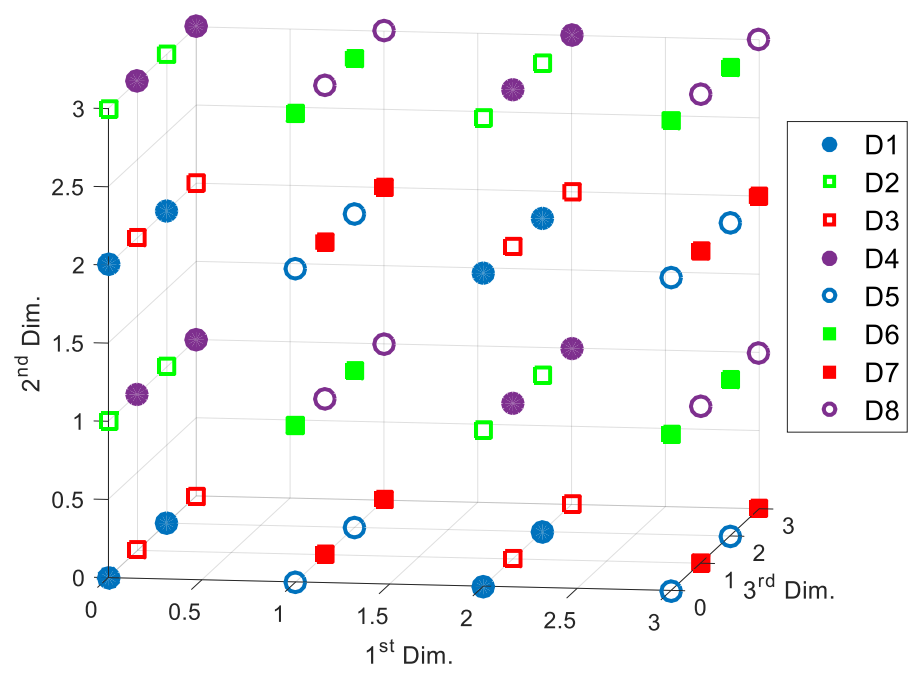

Figure 6.4. 3D PAM-4 TCM constellation representation by subsets

As mentioned above, the convolutional encoder has a 2-bit input and 3-bit output, resulting in a code rate of $2 / 3$. Since the $d_{\min }$ within a subset is equal to 2 and the $d_{\min }$ provided by the encoder should be larger than the one provided by set partitioning (as explained in the previous section), the number of states of the convolutional encoder has to be equal or larger than 8. An 8-state and a 16-state convolutional encoder are tested here (ensuring a $d_{\min }$ of 2.24 and 2.65 respectively [19]). The block diagrams of these encoders are displayed below. 


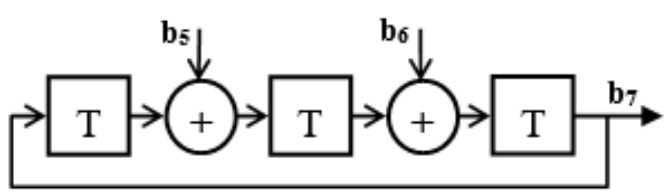

8-state encoder

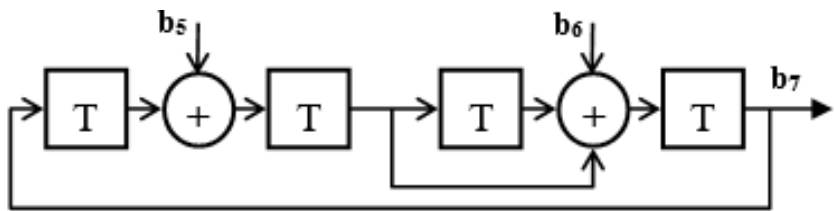

16-state encoder

Figure 6.5. Convolutional encoder block diagram

In order to test the performance of 4D PAM-4 TCM and the difference between the 8-state and 16-state convolutional encoder a simulation is performed, where the TCM scheme and regular PAM-4 are compared. The target is a $56 \mathrm{Gbps}$ BTB transmission at a wavelength of $1310 \mathrm{~nm}$. To achieve this bit rate the PAM-4 operates at 28 GBaud while the 4D PAM-4 TCM requires a transmission rate of 32 GBaud.

$$
B r_{4 D \text { PAM } 4 \text { TCM }}=\frac{b_{\text {total }}}{b_{\text {info }}} * B r_{P A M 4}=\frac{8}{7} * 28 \text { GBaud }=32 \text { GBaud }
$$

The 3-dB bandwidths of the modulator and of the PD are set to $18 \mathrm{GHz}$. The performance is evaluated in terms of receiver sensitivity. The results of this simulation are displayed below, in Figure 6.6. A 21-tap FFE is employed for equalization for both PAM-4 and 4D PAM-4 TCM. The optical noise is set to zero.

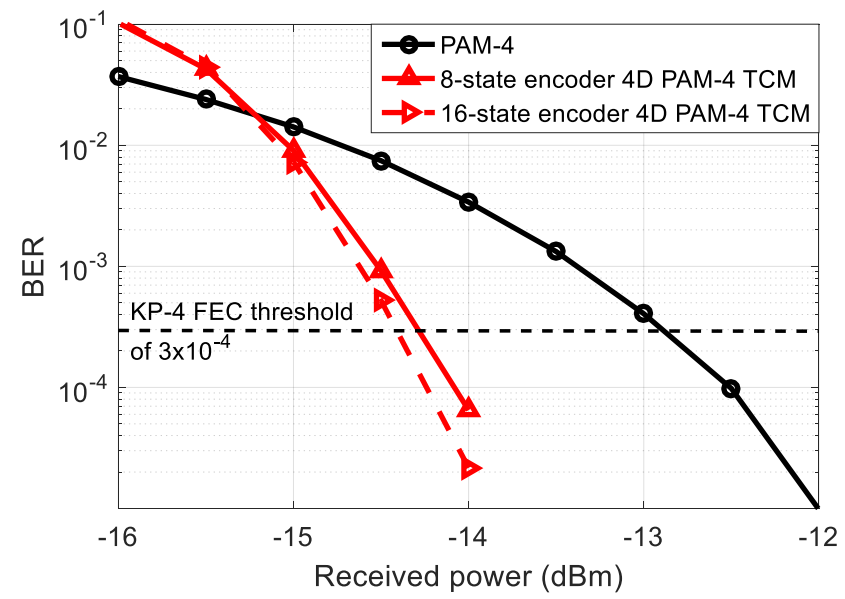

Figure 6.6. Simulation comparison between PAM-4 and 8-state and 16-state convolutional code $4 D$ PAM-4 TCM (at 56 Gbps, BTB)

The results for both types of convolutional encoder are represented at a bit rate of $56 \mathrm{Gbps}$. The 16-state encoder is expected to provide some gain over its 8-state counterpart. For the 4D PAM-4 TCM we observe a small gain of roughly $0.1 \mathrm{~dB}$, in favor of the 16 -state encoder. Due to this small gain it can be concluded that, when talking about a practical implementation, upgrading to a 16state encoder from an 8-state one is not worth the extra complexity. Both versions of the 4D PAM4 TCM outperform the classic PAM-4 at BERs below $10^{-2}$. At the KP-4 FEC limit $\left(3 \times 10^{-4}\right)$ TCM improves sensitivity by $1.4 \mathrm{~dB}$ (when using the 8 -state encoder) to $1.5 \mathrm{~dB}$ (when using the 16-state 
encoder). As the BER decreases so does the gain provided by TCM. This is to be expected when employing a modulation format which integrates FEC elements, explaining also why above $10^{-2}$ 4D PAM-4 TCM performs worse than PAM-4.

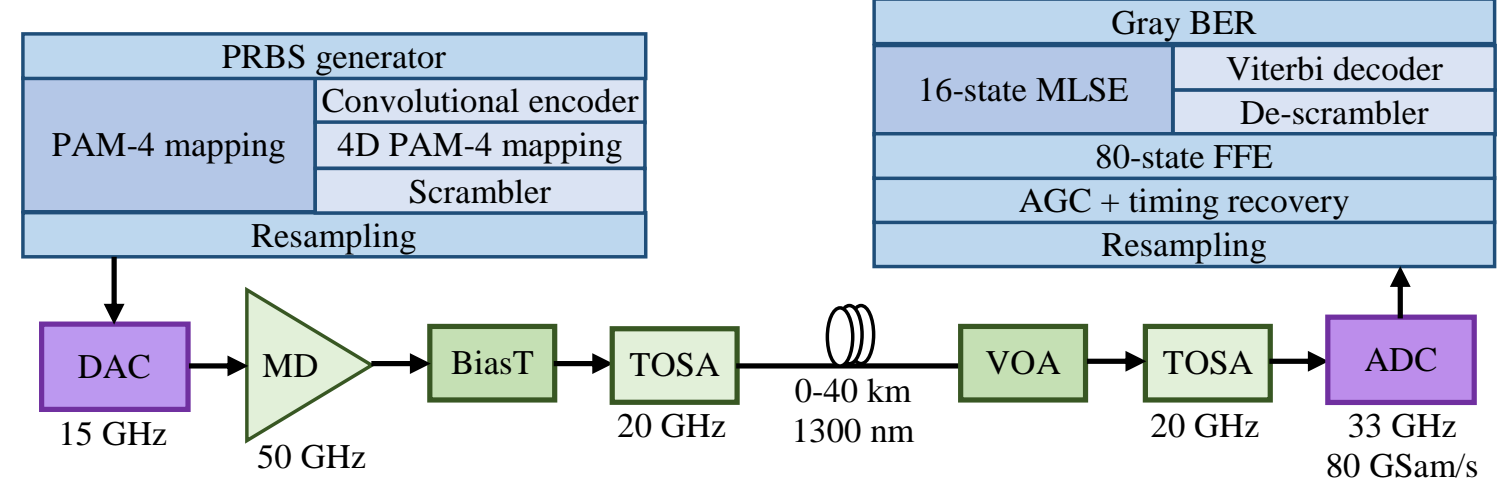

Figure 6.7. 4D PAM-4 TCM vs. PAM-4 experimental setup block diagram

In order to validate the simulation results a series of experiments was performed. The experimental configuration used to assess the TCM performance is shown in Figure 6.7. A pseudo-random nonreturn-to-zero data sequence of $2^{15}$ symbols is generated offline in MATLAB and is loaded to a DAC with 8-bit resolution and a 3-dB bandwidth of $15 \mathrm{GHz}$. The optical link consists of a TOSA, an SSMF spool, and a ROSA. The ROSA input optical power is controlled by a VOA. The received data are captured by a real-time oscilloscope operating at $80 \mathrm{GSamples/s}$ and are processed offline using DSP. In the DSP part, the signal is first resampled, amplified, and then a timing recovery algorithm [51] is used to compensate for the clock frequency offset between the transmitter and receiver clocks. The data are partly recovered using a simple FFE (operating in blind mode and employing the MMA algorithm) that compensates for ISI. Two different TCM decoders (one for the 8-state convolutional code and the other for the 16-state) based on a trellis search (i.e. the Viterbi algorithm) are used to find the most probable transmitted symbol sequence [102]. The decoded PAM-4 symbols (synchronized in groups of four symbols) are de-mapped into bits by using a look-up table (the table consists of 256 entries where four symbols provide seven information bits). The Gray BER is measured at the output of the TCM decoder to evaluate the system's performance in the BTB case as well as after transmissions of $10 \mathrm{~km}, 20 \mathrm{~km}, 30 \mathrm{~km}$, and $40 \mathrm{~km}$.

The performance of the two TCM schemes (i.e. the TCM with an 8- and a 16- states convolutional encoder) is evaluated before and after the trellis decoder for the BTB scenario. Results are presented in Figure 6.8(a), where we refer to the 8-state TCM as TCM1 and to the 16-state TCM as TCM2. Although a sensitivity gain of around $0.1 \mathrm{~dB}$ in favor of TCM2 is expected (as seen in simulations), the experimental results show that both coding methods perform nearly the same. Thus, we infer that the relatively low gain of TCM2 over TCM1 shown in Figure 6.8(a) does not justify the extra complexity imposed by the 16-state convolutional code. In the same figure the BER performance for PAM-4 is also presented. In that case, an 81-tap FIR equalizer and a 16-state 
MLSE are employed at the receiver's DSP to obtain the results. As can be observed, PAM-4 TCM outperforms the conventional PAM-4 scheme by $2 \mathrm{~dB}$ at BERs lower than $10^{-5}$. Note here, that only an 81-taps FIR filter is used before the decoder for the TCM case. Moreover, a tail extrapolation algorithm [108] is used to extend the curves from Figure 6.8(a) down to a BER of $10^{-15}$ as shown in Figure 6.8(b). Here we can see that PAM-4 TCM provides a $4 \mathrm{~dB}$ sensitivity gain compared to PAM-4 at BER values equal to $10^{-15}$. The sensitivity requirements of 4D PAM4 TCM are observed before and after the Viterbi decoder in both Figure 6.8(a) and Figure 6.8(b). Before the decoder the TCM scheme exhibits a loss of about $1 \mathrm{~dB}$ when compared with PAM-4; this is due to the higher baud rate required by the 4D PAM-4 TCM (32 GBaud instead of 28 GBaud). After decoding the performance is greatly improved and TCM outperforms PAM-4, as explained above.
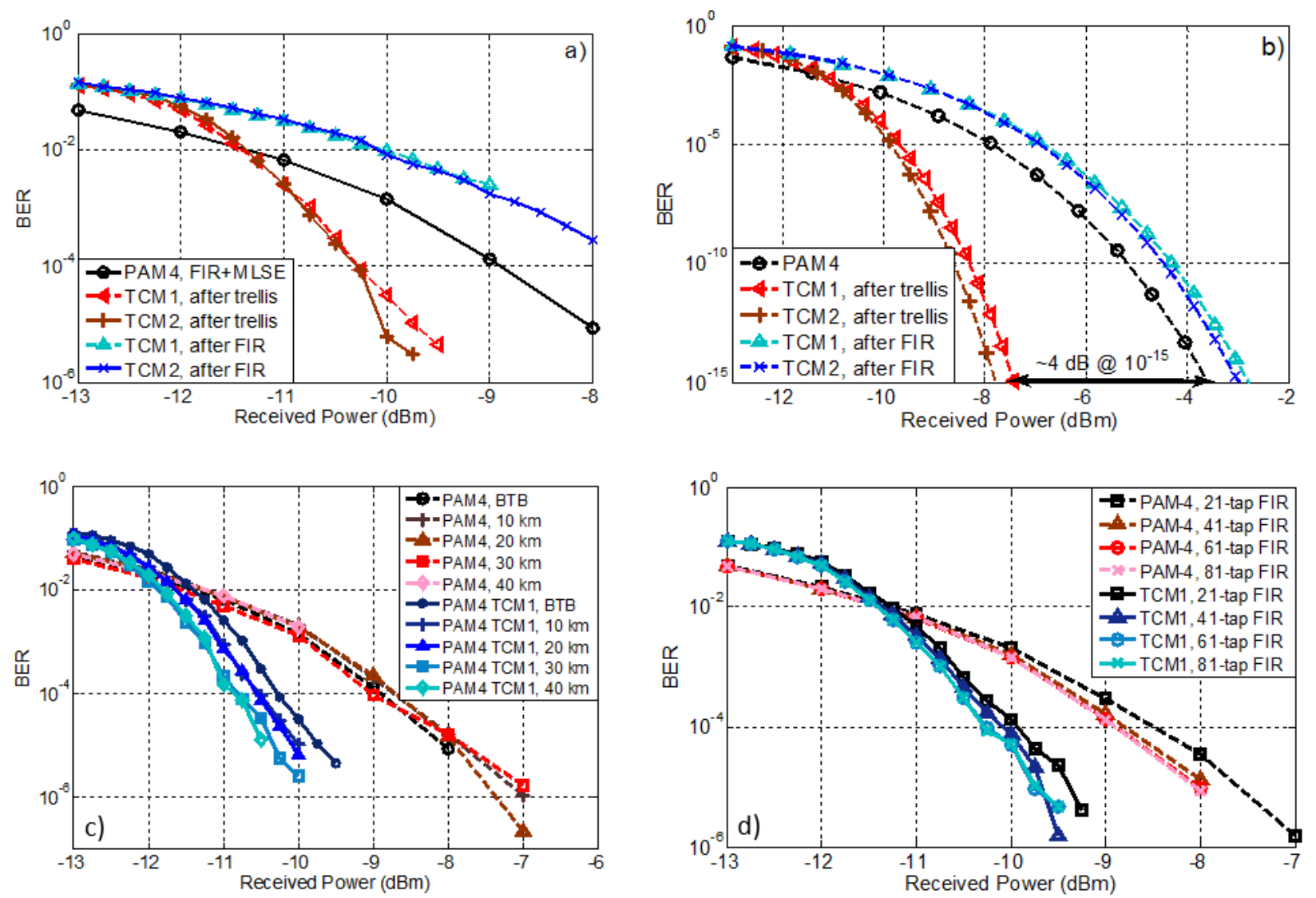

Figure 6.8. Performance comparison between 8-state and 16- state TCM PAM-4 (before and after trellis decoder) and PAM-4. BER curves are obtained by processing the experimental data (a) and by tail extrapolation (b). BER curves for PAM-4 and 8-state TCM for BTB, 10, 20, 30 and $40 \mathrm{~km}(\mathrm{c})$ and for 21, 41, 61 and 81-tap FFEs (d)

Next, the performance of the 8-state 4D PAM-4 TCM and that of the conventional PAM-4 scheme are compared after transmissions over $10 \mathrm{~km}, 20 \mathrm{~km}, 30 \mathrm{~km}$ and $40 \mathrm{~km}$ and the results are presented in Figure 6.8(c). Due to the TOSA's emission wavelength being $1300 \mathrm{~nm}, \mathrm{CD}$ does not have a significant impact on the transmission, leading to negligible variation in performance for different 
transmission distances. At $40 \mathrm{~km}$ the attenuation in the fiber limits the maximum achievable power at the receiver to $-10 \mathrm{dBm}$. As we can see from Figure 6.8(c), in this case, 4D PAM-4 TCM can provide BERs below the KP-4 pre-FEC threshold of $3 \times 10^{-4}$, while transmission is not feasible for PAM-4 for the same sensitivity requirements.

Finally, the influence of the number of taps in the FIR filter on the system's performance is investigated. In Figure 6.8(d) the BER curves when using FIRs with 21, 41, 61 and 81 taps are presented, for both TCM 8-states PAM-4 and conventional PAM-4. The curves for 61-tap and 81tap FFE are almost identical leading to the conclusion that BER cannot be further improved by increasing the number of taps of the FIR beyond 61. Even with a 21-tap FIR the performance is quite close to optimum.

The 4D PAM-4 TCM is shown to be a promising candidate for next generation $400 \mathrm{Gbit} / \mathrm{s}$ data center interconnects. In the experimental results presented here it is proven that the TCM scheme significantly improves power sensitivity when compared to conventional PAM-4 proposed so far in literature for links of up to $40 \mathrm{~km}$ without the need for optical amplifiers. At the KP-4 pre-FEC threshold the investigated scheme enables sensitivity gains of more than $1 \mathrm{~dB}$. Without FEC the estimated potential gain of $4 \mathrm{~dB}$ is observed at a BER of $10^{-15}$.

\subsection{The 4D PAM-5 TCM scheme}

The TCM scheme presented in the previous sub-chapter requires an increase in baud rate, when compared with its parent modulation (i.e. PAM-4), in order to achieve the same bit rate as the classic PAM-4. In this section another TCM scheme is introduced, which does not require higher baud rate. This is achieved by increasing the number of levels of the output modulation from four to five. We will refer to this modulation scheme as 4D PAM-5 TCM. The performance of this TCM variation is evaluated here and compared with the performances of PAM-4 and 4D PAM-4 TCM.

The PAM-4 modulation maps two bits onto one symbol, resulting in a two bits/symbol transmission ratio. The 4D PAM-4 TCM maps seven bits onto four symbols, thus requiring a baud rate 8/7 times higher than the PAM-4 scheme. 4D PAM-5 TCM has an output made out of four PAM-5 symbols, resulting in a 625 points constellation (see equation (86)). Such a constellation can accommodate a total of nine bits, one more than the 4D PAM-4 constellation. Thus, if keeping the same convolutional encoder as in the case of 4D PAM-4 TCM, a total of eight information bits can be transmitted by each 4D PAM-5 super-symbol, instead of seven, as it was the case for the 4D PAM-4 TCM. The transmission rate of the 4D PAM-5 TCM is therefore equal to that of PAM4, i.e. 2 bits/symbol. The drawback is a lower noise tolerance due to the increase in the number of levels. The generation of the 4D PAM-5 TCM super-symbols is illustrated in Figure 6.9. 


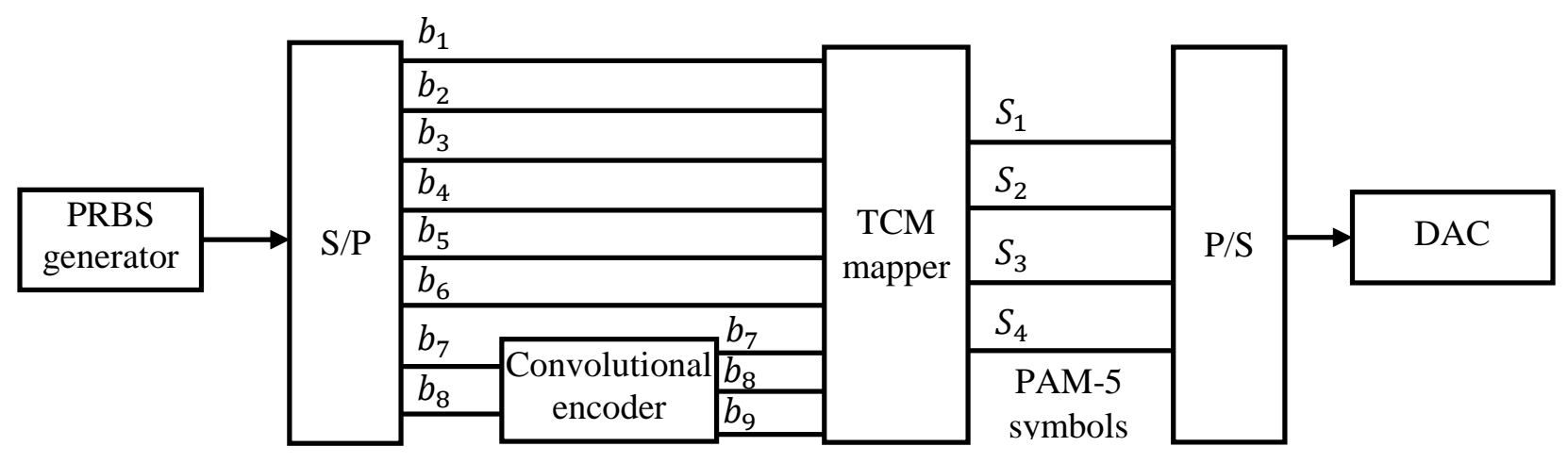

Figure 6.9. 4D PAM-5 TCM generation block diagram

The partitioning process is very similar to the one presented in the previous sub-chapter for 4D PAM-4 TCM. The PAM-4 levels are again organized into two groups, X and Y (even and odd). X comprises of levels $\{0,2,4\}$ and $Y$ of levels $\{1,3\}$. The same convolutional encoders are employed here (i.e. the 8-state and 16-state encoder from Figure 6.5), meaning that again the full constellation is split into eight subsets based on the even and odd groups, as shown in Table 6. Since now the constellation has 625 points, the subsets are also larger. Because the sizes of $\mathrm{X}$ and $\mathrm{Y}$ are no longer equal, the subsets will also vary in the number of points. Only 512 points are required to map all the bits at the input of the TCM mapper, thus the size of each subset is reduced down to 64 points. In order to assure the best noise tolerance only the highest power symbols of each subset are removed. For example if we consider $D_{2}=\mathrm{XXYY}+\mathrm{YYXX}$ which has a total of 72 points, 8 points have to be removed to bring it down to 64 and these eight super-symbols should be the ones with the highest power $-\{4,4,3,3\},\{3,3,4,4\},\{4,4,3,1\},\{3,1,4,4\},\{4,4,1,3\},\{1,3,4,4\},\{4,4,1,1\}$ and $\{1,1,4,4\}$.

Table 6. 4D PAM-5 TCM subsets $\left(D_{\overline{1,8}}\right)$

\begin{tabular}{|c|c|c|c|}
\hline Subset & 4-group super-symbol representation & Number of points & Pruned down to \\
\hline$D_{1}$ & $\mathrm{XXXX}+\mathrm{YYYY}$ & 97 & 64 \\
\hline$D_{2}$ & $\mathrm{XXXY}+\mathrm{YYYX}$ & 78 & 64 \\
\hline$D_{3}$ & $\mathrm{XXYX}+\mathrm{YYXY}$ & 78 & 64 \\
\hline$D_{4}$ & $\mathrm{XXYY}+\mathrm{YYXX}$ & 72 & 64 \\
\hline$D_{5}$ & $\mathrm{XYXX}+\mathrm{YXYY}$ & 78 & 64 \\
\hline$D_{6}$ & $\mathrm{XYXY}+\mathrm{YXYX}$ & 72 & 64 \\
\hline$D_{7}$ & $\mathrm{XYYX}+\mathrm{YXXY}$ & 72 & 64 \\
\hline$D_{8}$ & $\mathrm{XYYY}+\mathrm{YXXX}$ & 78 & 64 \\
\hline
\end{tabular}

In order to better illustrate the subsets and the full constellation, a 3D PAM-5 TCM constellation is shown in Figure 6.4 (not 4D since only 3D can be represented in space). 


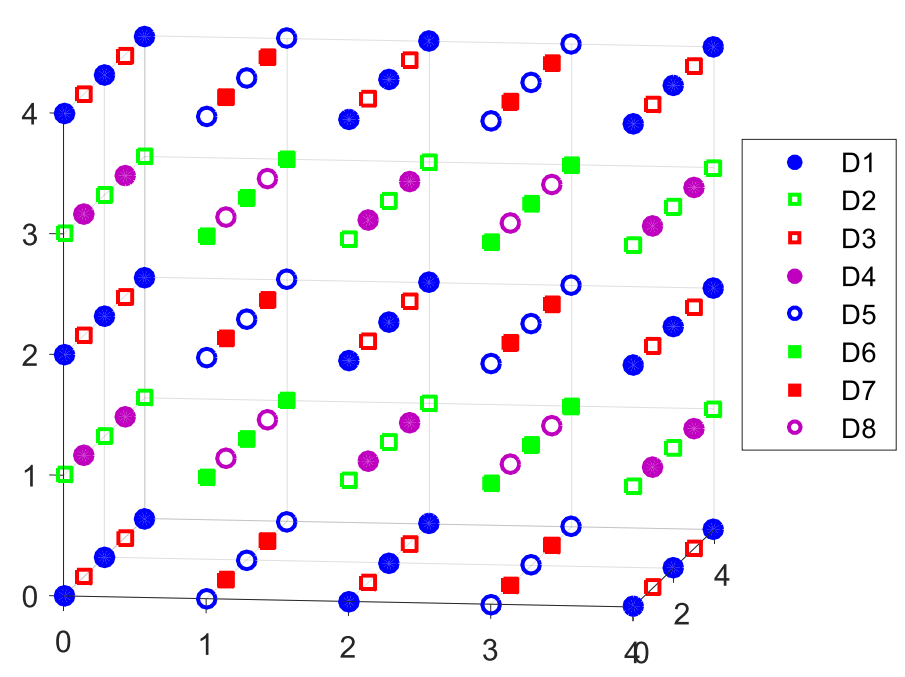

Figure 6.10. 3D PAM-5 TCM constellation representation by subsets

Using the experimental setup presented in Figure 6.11 the performance of 4D PAM-5 TCM is evaluated in order to assess the feasibility of using TCM in next generation 400G WDM optical networks. The results are also compared with the performance of 4D PAM-4 TCM and PAM-4. The capabilities of the 8-state and 16-state convolutional encoders are tested in the 4D PAM-5 scenario. The three modulations (4D PAM-4 and PAM-5 TCM and PAM-4) are also analyzed at different transmission distances and varying baud rates.

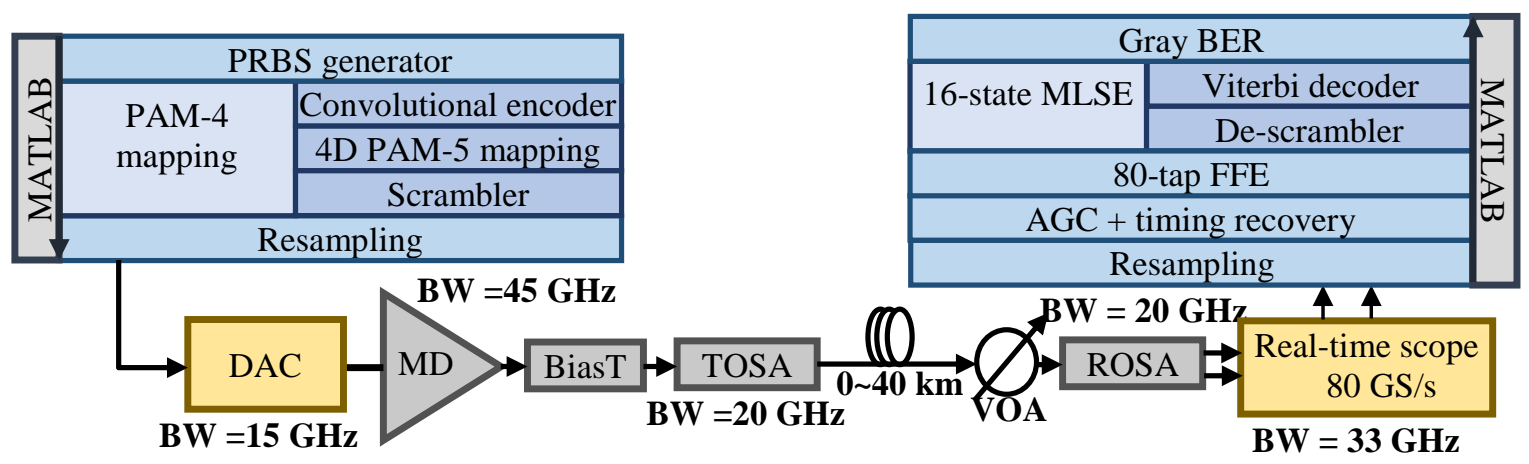

Figure 6.11. Experimental setup for testing the $4 D$ PAM-5 TCM

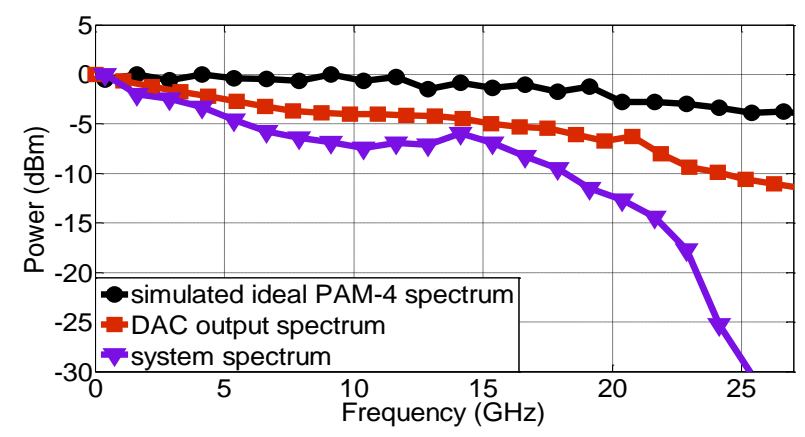

Figure 6.12. The transfer function of a simulated PAM-4 signal, of the DAC, and of the overall system 
At the transmitter side DSP is needed to generate the more complex TCM and to prepare the data for the DAC. Firstly, a PRBS is generated using the MATLAB 'rand' function. A DAC sequence length of 43008 is chosen, which ensures a proper number of samples (multiple of 128, see chapter 2.1.1) for any integer baud rate ranging from 28 to 56 GBaud. For TCM the PRBS generator is followed by a convolutional encoder (8- or 16-state). Next, the bits are mapped onto their corresponding constellation depending on the desired modulation format (PAM-4, 4D PAM-4 TCM or 4D PAM-5 TCM). In the case of PAM-5 TCM the modulation symbols are scrambled to ensure the symmetry of the signal (i.e. symmetry of the probabilities of the symbols) around the 0 level (as required by the FFE). This is done by multiplying the symbol sequence with another pseudo-random sequence of $1 \mathrm{~s}$ and $-1 \mathrm{~s}$ with the same length. Finally, the data is resampled in order to provide the correct number of samples/symbol required by the DAC, given a certain target baud rate. This data is converted into an electrical signal with the help of an 8-bit DAC. The 3-dB bandwidth of the DAC is lower than $15 \mathrm{GHz}$ and its transfer function can be seen Figure 6.12. The amplitude of the signal at the output of the DAC is approximately $400 \mathrm{mV}$. However, the optimum amplitude required by the modulator is around $2 \mathrm{~V}$. As a result, a MD is required to amplify the signal at the output of the DAC. In order to adjust the bias point a biasT is employed. The laser and the modulator are integrated together into a $20 \mathrm{GHz} 3-\mathrm{dB}$ bandwidth TOSA, which operates at $1300 \mathrm{~nm}$ wavelength. The optical link consists of 0 to $40 \mathrm{~km}$ of SSMF. A VOA is added before the receiver in order to adjust the power of the optical signal. The conversion of the optical signal back into the electrical domain is done by a ROSA with differential output and a 3-dB bandwidth of around $20 \mathrm{GHz}$. It is comprised of a photodiode and a TIA. The ROSA achieves optimum performance at a received power of around $-3 \mathrm{dBm}$ and further increasing the power will saturate the receiver photodiode. A $33 \mathrm{GHz}$ bandwidth real-time scope is used to capture offline data at a rate of 80 GSamples/s.

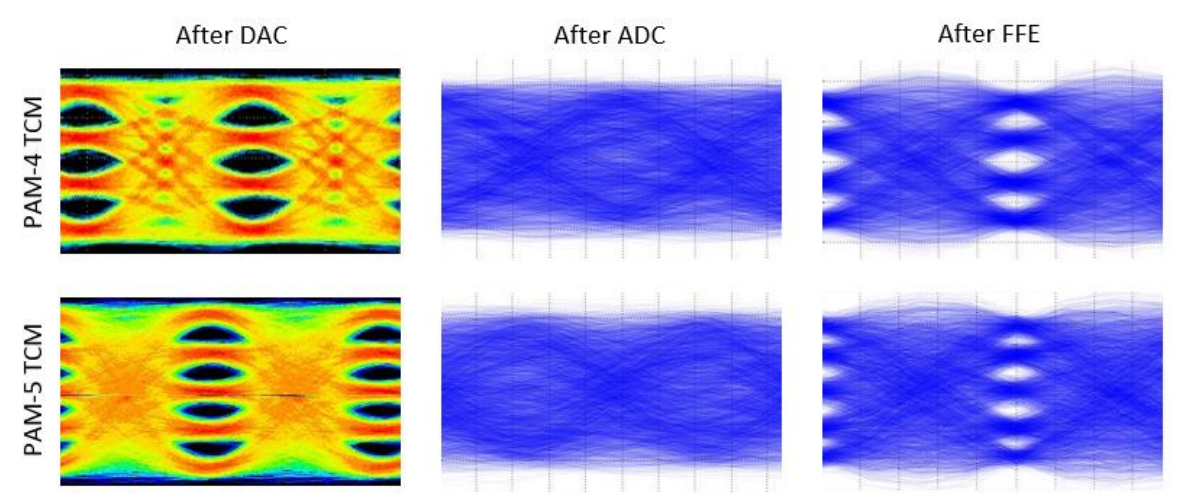

Figure 6.13. Eye diagrams of 4D PAM-4 and PAM-5 TCMs, after DAC, after ADC and after FFE. The eyes presented in this figure are obtained for a 56 Gbps, BTB transmission

The performance of the system is severely degraded, as can be observed from the eye diagrams in Figure 6.13. While at 28 GBaud the BER might be below the pre-FEC threshold, at higher baud rates this is no longer the case. Powerful algorithms are required to bring the BER down to acceptable levels. At the receiver side the data is prepared for equalization. First, the received data 
is resampled to two samples/symbol. The DAC introduces some clock phase variations which affect the performance of the FFE. For this reason timing recovery, based on the Mueller and Müller algorithm [51], is performed after data resampling. Out of the two samples/symbol only one is used by the MM algorithm. Following the timing recovery, an AGC module is employed in order to remove the DC component and to adjust the signal swing. Two DSP algorithms are implemented, the FFE and the MLSE. The FFE is used by all three modulation types that are investigated in this section. To compute the tap coefficients of the FFE, the MMA algorithm [109] is employed. At high baud rates this equalizer alone is not enough to bring the BER of the system below the pre-FEC limit. The second equalizer implemented here is the MLSE equalizer. In the case of PAM-4 modulation the typical MLSE based on Euclidian distance metrics and the Viterbi algorithm [110] is used. The Viterbi algorithm outputs hard values but TCM, which also employs the Viterbi algorithm in its decoder, requires soft inputs. This means the MLSE algorithm needs to be modified to output soft values in order to work in conjunction with TCM and thus the classic MLSE hard output algorithm is used only for PAM-4. For the TCM schemes the SOVA MLSE solution is employed. The last step after equalization is to calculate the Gray BER.

Due to the noise and bandwidth limitations of the experimental setup, the hard decision BER of the offline data is typically higher than $10^{-2}$, which puts it well above the pre-FEC limit. To bring the BER down to acceptable levels equalization is required. First the effects of FFE are analyzed. The ratio between performance and complexity for FFEs is controlled by the number of taps used. The higher the number of taps the better the FFE will perform. If the number of taps is equal or greater than the channel memory, further increasing the number of taps will not improve the BER anymore. We evaluate the performance of a FFE with different number of taps, ranging from 4 to 100 (i.e. 4, 8, 12, 20, 40, 60, 80, 100) for TCM and from 20 to 100 (i.e. 20, 40, 60, 80, 100) for PAM-4. The results of this evaluation can be seen in Figure 6.14(a) for the PAM-4, 4D PAM-4 TCM and 4D PAM-5 TCM schemes, where the required power to go below the pre-FEC threshold is shown, for the different modulation/number of FFE taps combinations. It can be observed that an 80-tap FFE delivers the best results and that increasing the number of taps to 100 or more does not bring any noticeable gain. In the case of TCM we also notice that for a 20-tap FFE the BERs are really close to those obtained with an 80-tap FFE and only when using less than 20 taps does the performance start to drop significantly. Thus, we can conclude that the best performancecomplexity ratio is provided by a 20-tap FFE. However, in the case of PAM-4, using only 20 taps will reduce the power sensitivity by about $0.4 \mathrm{~dB}$ at BERs below the KP4 pre-FEC threshold. In the interest of presenting the best results, the 80-tap FFE is used throughout the rest of this section of the thesis. 

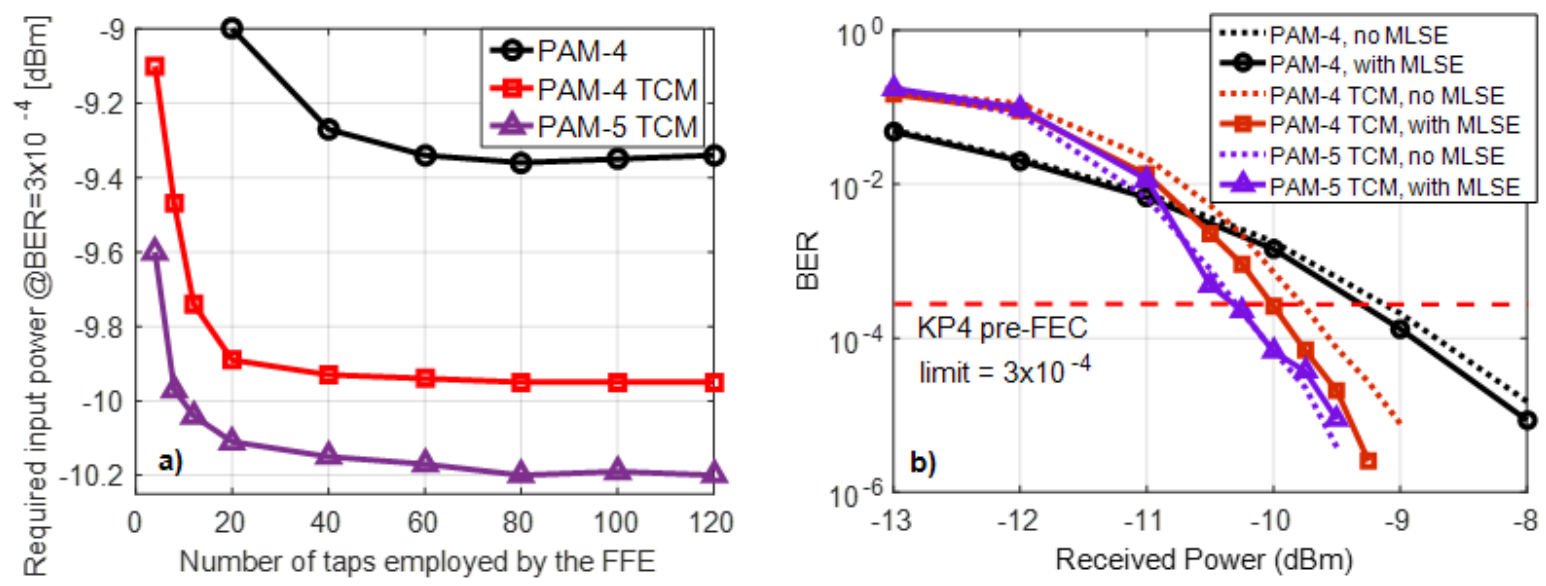

Figure 6.14. The number of FFE taps versus the input power required to obtain a BER of $3 \times 10^{-4}$ for PAM-4, PAM-4 TCM and PAM-5 TCM schemes (a) and Comparison of performance for TCM and PAM4 with and without MLSE (b)

The MLSE is a powerful algorithm used to compensate for bandwidth limitation or residual CD. Its main drawback is its complexity, which increases exponentially with the number of memory symbols taken into account by the Viterbi MLSE decoder. Unlike the PAM-4 modulation where MLSE with hard output can be used, the MLSE algorithm used for TCM must output soft values in order for the TCM decoder to work properly. For this purpose we employ the SOVA MLSE [111]. In this experiment we use a memory length of 2, resulting in 16 states for a PAM-4 signal or 25 states for a PAM-5 one. In Figure 6.14(b) the results for the 16/25-state MLSE at a bit rate of $56 \mathrm{Gbps}$ are shown. With dotted lines are represented the BER curves after an 80-tap FFE without using any MLSE and the continuous lines show the same curves when the MLSE equalizer is added. We observe that for PAM-5 TCM and PAM-4 modulations MLSE does not bring any significant gain, which means that the FFE is able to compensate for most of the ISI introduced by bandwidth limitations. On the other hand for the PAM-4 TCM we start seeing an improvement when the MLSE is used. This is due to the fact that the PAM-4 TCM has a bit/symbol ratio of 7/4 and in order to reach a bit rate of $56 \mathrm{Gbps}$, a baud rate of $32 \mathrm{Gbaud}$ (instead of 28 GBaud required by PAM-4 and PAM-5 TCM) is necessary. This means that the PAM-4 TCM is affected more by bandwidth restrictions and as a result the FFE cannot correct all ISI anymore. If the bit rate is increased the MLSE starts improving power sensitivity for PAM-4 and also for 4D PAM-5 TCM.

Just like in the previous section, the experimental setup here operates at a wavelength of $1300 \mathrm{~nm}$, which implies that the influence of $\mathrm{CD}$ on the quality of the optical signal should be very small. In order to test this supposition the 4D PAM-5 TCM was transmitted BTB and over links of 10, 20, 30 and $40 \mathrm{~km}$ and its performance in terms of receiver sensitivity is compared with that of $4 \mathrm{D}$ PAM-4 TCM and PAM-4. The results of these experiments are displayed below, in Figure 6.15(a)(e). 

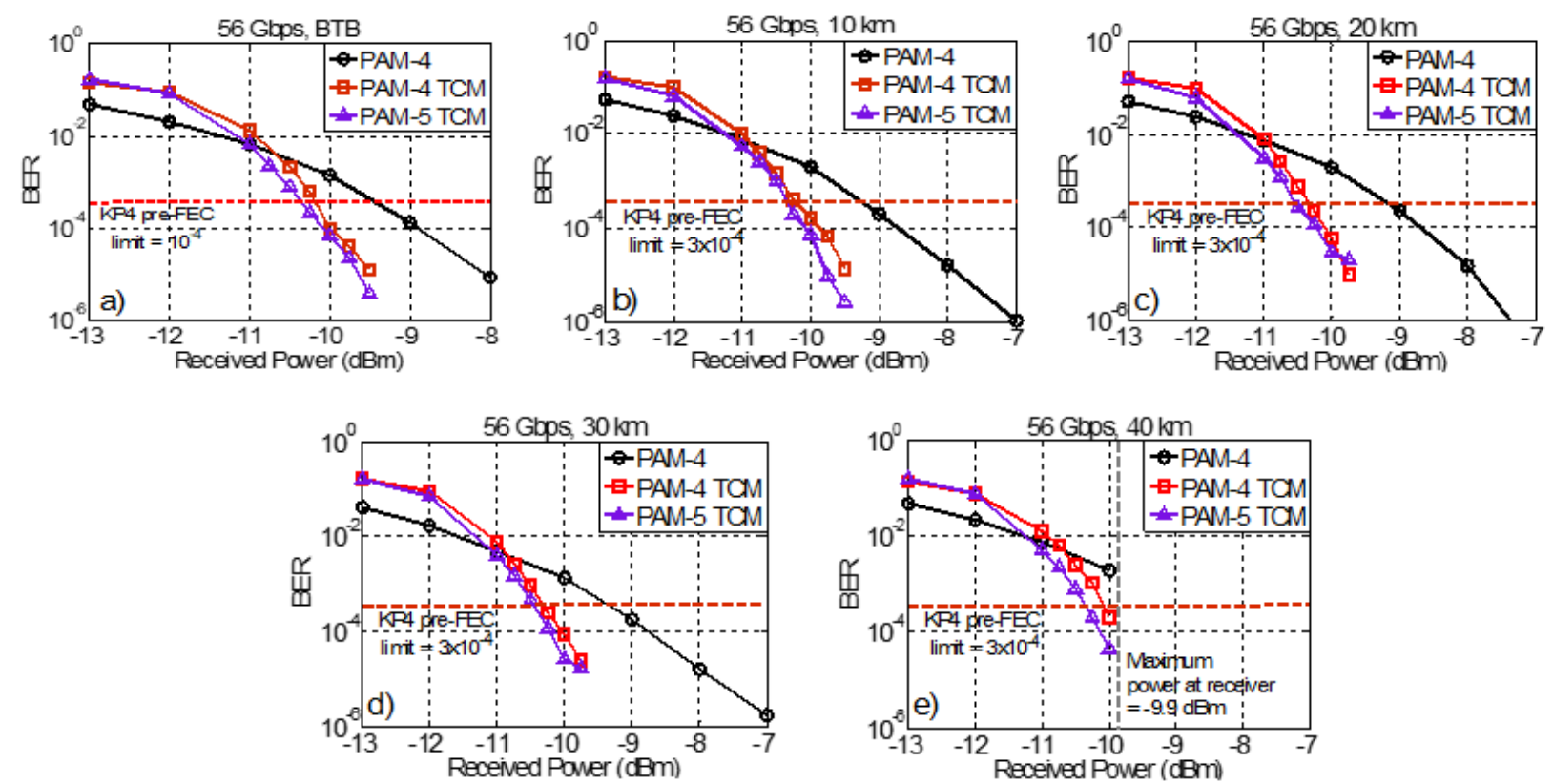

Figure 6.15. Comparison of performance of PAM-4 TCM (squares), PAM-5 TCM (triangles) and of PAM-4 (circles) for BTB (a) and links of $10 \mathrm{~km}(\mathrm{~b}), 20 \mathrm{~km}(\mathrm{c}), 30 \mathrm{~km}(\mathrm{~d})$ and $40 \mathrm{~km}(\mathrm{e})$ at $56 \mathrm{Gbps}$

It can be observed that the results remain approximately the same, no matter the link length, just as expected. The downside is that the attenuation in the link is higher than it would normally be at wavelengths of around $1550 \mathrm{~nm}$. The power of the optical signal at the output of the TOSA is 4 $\mathrm{dBm}$. Every $10 \mathrm{~km}$ of SSMF will attenuate the signal by about $3.5 \mathrm{dBm}$. For a $40 \mathrm{~km}$ link the maximum achievable power is $-9.9 \mathrm{dBm}$. If we take another look at Figure 6.15(a)-(e) we see that, in order to reach BERs below the KP4 pre-FEC limit of $3 \times 10^{-4}$, PAM-4 TCM and PAM-5 TCM require a minimum power of $-10 \mathrm{dBm}$ and of $-10.25 \mathrm{dBm}$ respectively, while PAM-4 requires at least $-9 \mathrm{dBm}$. This means that TCM enables a $40 \mathrm{~km}$ transmission, where with PAM-4 only $30 \mathrm{~km}$ are achievable. Another phenomena that can be observed in Figure 6.15 is that performance at 20 and $30 \mathrm{~km}$ is slightly better (i.e. $0.2-0.3 \mathrm{~dB}$ ) than in the BTB, 10 and $40 \mathrm{~km}$ cases. This is due to the negative chirp of the TOSA's modulator (the CD sign is positive).

The two most probable implementations of PAM-4 in the next generation 400G optical networks are $8 \lambda \times 50$ and $4 \lambda \times 100$ Gbps. It has been showed in this and previous sections that TCM outperforms PAM-4 at a data rate of 56 Gbps, making it a suitable candidate for an $8 \lambda \times 50$ Gbps transmission. Next the three modulations are evaluated experimentally at higher bit rates (i.e. 64, 72, 80, 88, 96, 104 and 112 Gbps), where the corresponding BER curves are shown in Figure 6.16(b)-(h). For all these rates the performance of the different modulation formats is evaluated for BERs that are below the KP4 pre-FEC limit of $3 \times 10-4$. 

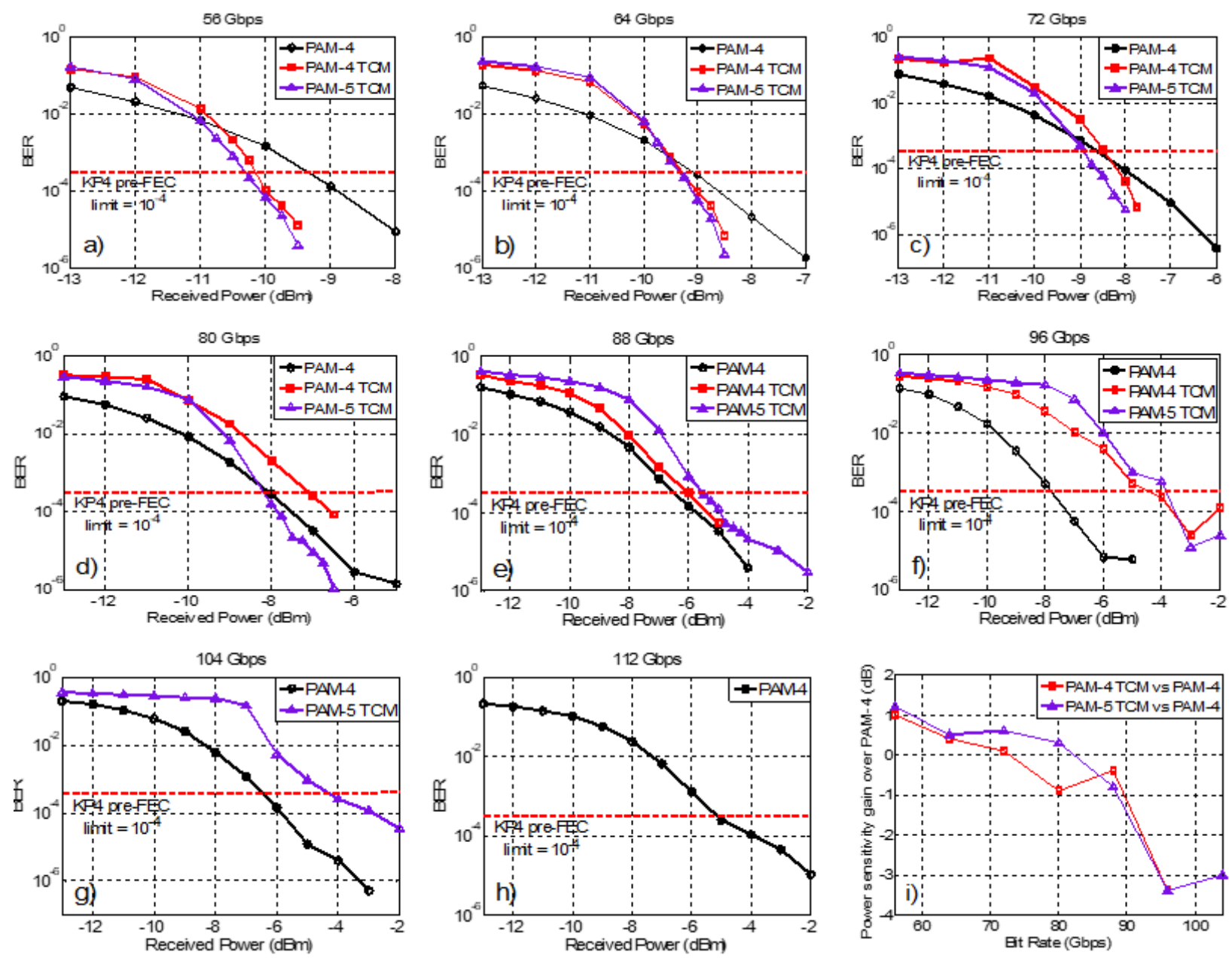

Figure 6.16. Power sensitivity comparison between 4D PAM-4 and PAM-5 TCM and PAM-4 at 56 (a), 64 (b), $72(\mathrm{c}), 80(\mathrm{~d}), 88(\mathrm{e}), 96(\mathrm{f}), 104(\mathrm{~g}), 112 \mathrm{Gbps}(\mathrm{h})$, and the gain of TCM over PAM-4 at bit rates between 56 and 112 Gbps (i)

At 64, 72, and 80 Gbps the performance balance stays more or less the same, with 4D PAM-5 TCM still being the best option. However, we can see that the gain in terms of power sensitivity provided by TCM slowly decreases as we increase the bit rate. At 80 Gbps PAM-5 TCM will improve upon PAM-4 by only 0.3-0.5 dB, while 4D PAM-4 TCM is no longer capable of outperforming PAM-4. At 88 Gbps PAM-4 performs best out of the three modulation schemes, improving power sensitivity by about $0.4 \mathrm{~dB}$ when compared to 4D PAM-5 TCM. When we further increase transmission speed to $96 \mathrm{Gbps}$ we observe that the MMA used to compute the tap coefficients of the FFE is no longer able to converge to the correct tap values. The MMA is set up to detect a 4-level (for PAM-4 TCM and PAM-4) or a 5-level (for PAM-5 TCM) signal. Because of the very limited bandwidth of the experimental setup ISI is present and thus, for bit rates of 96 Gbps or higher, we receive a DB signal. This means that any received symbol is in fact the sum of the corresponding transmitted symbol and the symbol transmitted previously [83], resulting in a 7-level (for PAM-4 and PAM-4 TCM) or a 9-level (for PAM-5 TCM) signal. In order for the FFE to work properly we modify the MMA to detect seven or nine levels for bit rates of 96 Gbps or 
higher. No extra encoding is required at the transmitter side as the MLSE equalizer is capable of decoding the DB signal on its own. When switching to a DB signal the difference in performance between PAM-4 and TCM gets much larger in the favor of PAM-4. At 96 Gbps a gap of more than $3 \mathrm{~dB}$ between TCM and PAM-4 can be observed (in Figure 6.16(f)). When increasing the bit rate to $104 \mathrm{Gbps}$, the DB MMA algorithm is no longer able to converge properly in the case of PAM4 TCM and thus BERs below the KP4 pre-FEC threshold cannot be achieved. The same happens for both TCM schemes at 112 Gbps.

In Figure 6.16(i) the results from Figure 6.16(a)-(h) are summarized by plotting the power sensitivity differences between PAM-4 TCM and PAM-4 and between PAM-5 TCM and PAM-4. Here we can better observe two phenomena: the power sensitivity of TCM drops faster than that of PAM-4 when we increase the bit rate and that both TCM schemes suffer a major penalty in performance when switching to a DB MMA (i.e. rates of 96 Gbps or higher). The first problem that is stated in this paragraph has different roots for the two TCM schemes. In the case of 4D PAM-4 TCM, because the bits/symbol ratio is lower than that of PAM-4 (i.e. 1.75 instead of 2), a higher baud rate is required in order to achieve the same bit rate (i.e. baud rate PAM-4 TCM = 8/7 times PAM-4 baud rate). This makes 4D PAM-4 TCM more sensitive to bandwidth limitations. Its PAM-5 counterpart does not suffer from the same issue as it has the same bits/symbol rate as PAM-4. However, unlike the PAM-4 where we deal with a 4-level signal, PAM-5 TCM has five amplitude levels. When increasing data rates, the noise in the FFE also increases. Since PAM-5 TCM has an extra level, the performance of the FFE will degrade faster (when increasing data rate) than in the case of PAM-4. The second issue is caused by the way the SOVA decoder works in conjunction with DB signals. The soft-output MLSE equalizer uses the trellis path metrics (forward and backward) to compute the probability (this probability is the output of the algorithm) of all symbols at any given time. This will work best when the probabilities of the levels of the input signal (which is the output of the FFE) are equal. While this is true for a PAM-4 signal, when using DB this is no longer the case. Since PAM-4 does not require the use of SOVA only the TCM schemes are affected. Advanced DSP techniques like turbo equalization might help to improve TCM performance at high baud rates but due to their complexity cost they are not considered in this thesis. 


\section{Chapter 7}

\section{Multidimensional multilevel set-partitioned modulation}

The rapid growth of datacenters is currently the main driving force for faster non-coherent short reach optical networks. Research into IM-DD systems is focused on $100 \mathrm{Gbps}$ per wavelength (net rate) transmissions. The PAM-4 modulation scheme seems to be preferred [112], due to its relatively low complexity and power consumption (in comparison with DMT). Recently IM-DD transmissions of around 200 Gbps or higher have been demonstrated using PAM-4 [67], [113], DMT [114], [115], and OOK [65]. These works typically employ some very high bandwidth components developed in-house and/or they multiplex two or more DAC channels.

The goal of this chapter is to demonstrate bit rates higher than 200 Gbps, using however only commercially available components. These components will inevitably introduce severe bandwidth limitations in the transmission system, leading to ISI. By using a FFE the ISI can be almost completely removed [15]. Another limitation when employing standard commercially available solutions is the limited sample rate of the DACs, which can reach a maximum of 100 GSamples/sec [116]. This means that baud rates higher than 100 GBaud are not possible (without severe penalties) and thus PAM-4 cannot currently go beyond $200 \mathrm{Gbps}$. To solve this issue and also to relax the bandwidth requirements a modulation format based on PAM-8 instead of PAM-4 is proposed. From previous results (sub-chapter 5.3, Figure 5.6) it can be observed that PAM-4 and PAM-8 exhibit a so called BER floor (higher modulation order $=>$ higher BER floor), which is caused by the electrical noise. Traditional DSP approaches like FFE and MLSE cannot combat the noise. In order to mitigate its effects, we have to increase the Euclidean distance between the constellation points. In order to do this the most widespread approach is to use TCM [100], [117], which was presented in chapter 6 . There it has been showed that TCM does not provide any gain when used in DB transmissions. Since DB is an essential DSP technique in combating the effects of ISI, this means that TCM cannot be used effectively for 200 Gbps transmissions.

In this chapter a new multidimensional modulation scheme based on set-partitioning and intensity modulation is presented. In the first part of this chapter the main principles behind this modulation are introduced and its generation is explained. In the second part a specific multidimensional setpartitioning modulation, referred to as the 3D PAM-8 scheme, is shown to improve noise tolerance in both DB and non-DB transmission systems, both in simulation and experimentally. The experimental setup is built only out of commercially available components. 


\subsection{Generation of multidimensional set-partitioned modulation schemes}

We saw in the previous chapter that the TCM scheme has problems in heavily bandlimited systems and especially when DB signaling is employed. In order to achieve data rates of $200 \mathrm{Gbps}$ or more, DB is an indispensable tool. The main idea behind multidimensional set-partitioned modulation is to keep the set-partitioning and multidimensionality elements of TCM but remove the FEC (convolutional code) part, as illustrated in Figure 7.1.

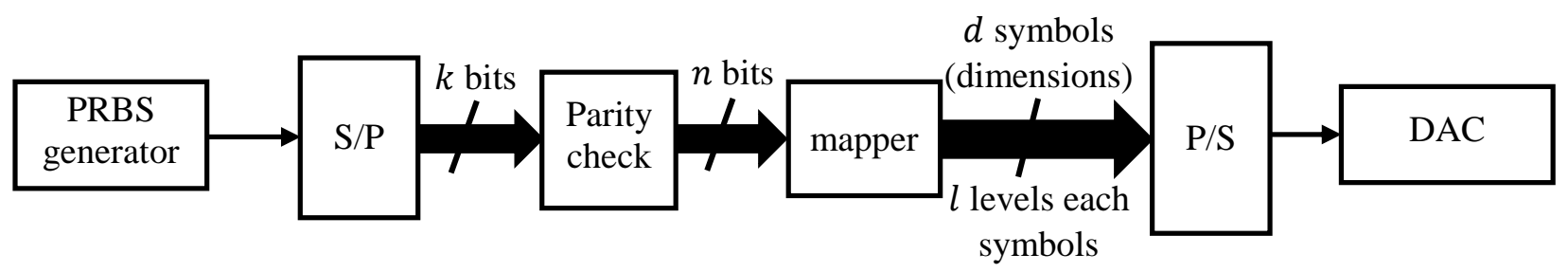

Figure 7.1. Multidimensional set-partitioned modulation generation block diagram

The PRBS to be transmitted is parallelized in groups of $k$ bits. These are then sent to a setpartitioning block which outputs $n$ bits, out of which $r$ bits are redundancy bits:

$$
n=k+r
$$

The maximum value of information bits in one group is limited by the parameters of the multidimensional super-symbols: $d$, the number of dimensions and $l$, the number of levels of the parent modulation. The number of constellation points can be calculated by using equation (86). As example let us consider a 4D PAM-4 version of the multidimensional set-partitioned modulation. Given this scheme $d=4$ and $l=4$, resulting in a full constellation of 256 points. A total of eight bits can be mapped onto this constellation, meaning that $n \leq 8$. Since $r \geq 1$, the number of information bits per one 4D PAM-4 super-symbol is $k=n-k \leq 7$.

Because FEC is no longer used here, the set-partitioning process is slightly different than in the case of TCM. The full constellation is going to be split into several subsets. The number of subsets is determined by $r$.

$$
N_{\text {subsets }}=2^{r}
$$

This time however these bits which encode the subset number are not encoded by a convolutional encoder like in the case of TCM and they will not carry any information. Instead they are used to ensure that the $k$ information bits are always mapped onto the same subset. Since the $d_{\min }$ within one subset is larger that $d_{\min }$ of the full constellation (see Figure 7.2), by placing all of the information in a single subset the noise tolerance of the modulation is increased. The trade-off of the set-partitioning technique is a decrease in spectral efficiency due to higher baud rate requirements. If the 4D PAM-4 modulation is considered again and $r$ is 1 , based on equation (87), the $d_{\min }$ is increased from 1 to 1.41 but the required baud rate is $8 / 7$ times higher. If $r$ is increased 
to 2 then the number of information bits is reduced to 6 , the $d_{\min }$ is increased from 1 to 1.73 and the required baud rate is $8 / 6$ times higher.

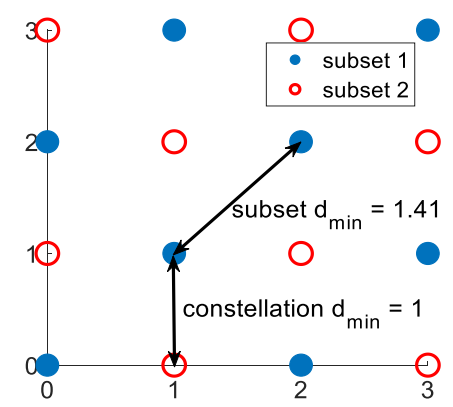

Figure 7.2. 2D PAM-4 constellation and set-partitioning when $r=1$

As the number of redundancy bits $r$ is increased, the relative $d_{\min }$ gain (the gain of $r$ minus the gain of $r-1$ ) is smaller and smaller while the baud rate penalty would increase at an accelerated rate, as can be seen from Table 7 (where a PAM-4 baud rate of 56 GBaud is assumed). As such it can be concluded that a $r$ of 1 is the optimum redundancy size for multidimensional set-partitioned modulation schemes and only this value of $r$ is considered in this thesis.

Table 7. The $d_{\min }$ and baud rate in relation with the number of redundancy bits for the $4 D P A M-4$ scheme

\begin{tabular}{|c|c|c|c|}
\hline redundancy & $d_{\min }$ & relative $d_{\min }$ gain & Baud rate [GBaud] \\
\hline$r=0 \rightarrow$ classic PAM-4 & 1 & 0 & 56 \\
\hline$r=1$ & 1.41 & 0.41 & 64 \\
\hline$r=2$ & 1.73 & 0.32 & 74.66 \\
\hline$r=3$ & 2 & 0.27 & 89.6 \\
\hline$r=4$ & 2.23 & 0.23 & 112 \\
\hline$r=5$ & 2.45 & 0.22 & 149.3 \\
\hline$r=6$ & 2.64 & 0.19 & 224 \\
\hline
\end{tabular}

When $r=1$, the redundancy bit can be calculated by using the following formula:

$$
b_{r}=\left[\begin{array}{llll}
b_{1} & b_{2} & \ldots & b_{k}
\end{array}\right] *\left[\begin{array}{c}
2^{(d-1) \% d} \\
2^{(d-2) \% d} \\
\vdots \\
2^{(d-k) \% d}
\end{array}\right]
$$

where $b_{r}$ is the redundancy bit and $d$ is the number of dimensions. We will refer to equation (91) throughout this chapter as the set-partitioning operation or simply set-partitioning. Once the redundancy bit has been computed the bits are mapped onto the $d$ PAM- $l$ symbol by reshaping the bits vector to a $d$ by $l$ matrix and converting it from binary to $l$-base, as demonstrated below:

$$
\left[\begin{array}{llll}
S_{1} & S_{2} & \cdots & S_{l}
\end{array}\right]=\operatorname{bi2de}\left(\operatorname{reshape}\left(\left[\begin{array}{llll}
b_{1} & b_{2} & \cdots & b_{n}
\end{array}\right], d, l\right)\right)
$$


To make these calculations clearer a numerical example is given next. Let us assume we want to transmit the following information bits: $\left[\begin{array}{lllllll}1 & 0 & 0 & 0 & 1 & 1 & 0\end{array}\right]$ as a 4D PAM-4 super-symbol. The redundancy bit can be calculated using equation (91):

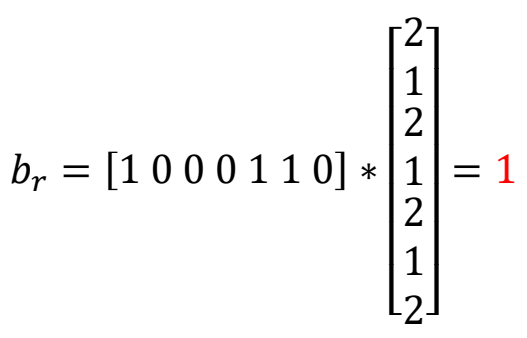

and the super-symbol can be computed with the help of equation (92):

$$
\left[\begin{array}{llll}
S_{1} & S_{2} & S_{3} & S_{4}
\end{array}\right]=\operatorname{bi2de}\left(\left[\begin{array}{ll}
1 & 0 \\
0 & 0 \\
1 & 1 \\
0 & 1
\end{array}\right]\right)=\left[\begin{array}{llll}
2 & 0 & 3 & 1
\end{array}\right]
$$

In previous chapters it has been shown that DB signaling is a key element of high-speed IM-DD optical bandlimited system. The main advantage of multidimensional set-partitioning modulation over TCM is that it can still provide a gain in DB systems. In order to enable the easy decoding of the DB signal at the receiver side equations (73)-(75) are employed. At the transmitter side, before the data is loaded into the DAC, differential encoding is applied (as described by (73)). The transmission system will act similar to a DB filter and thus we do not have to apply this filter in the digital domain. If we assume a DB 2D PAM-8 transmission then the received data has 15 levels, in comparison to just eight levels for a non-DB signaling. Thus, the received constellation also changes when DB is employed. However, although the number of levels is almost doubled, the ratio between the $d_{\text {min }}$ of the DB and non-DB versions of the constellation and subsets stays the same (i.e. 1.41), as can be seen in Figure 7.3. Since the DB constellation is more sensitive to noise (due to more levels) it is expected that DB gain of set-partitioning schemes will be smaller than the non-DB version.
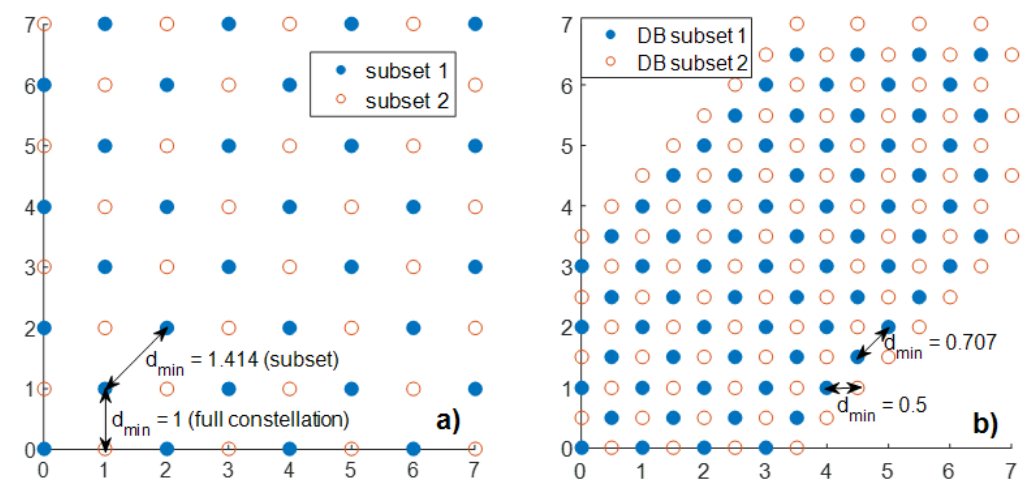

Figure 7.3. The 2D PAM-8 subsets (a) and 2D DB PAM-8 subsets after the receiver (b) 
Since the technique presented in this section requires only one bit of redundancy, no matter the dimensionality of the output super-symbol, it follows that the higher the dimensionality of the modulation the lower the baud rate required (but not lower or equal than the baud rate required by the parent modulation). For example, if we consider a 2D PAM-4 transmission with a target bit rate of $100 \mathrm{Gbps}$, the baud rate required to achieve it should be equal to the required baud rate of

the classic PAM-4 (i.e. 50 GBaud) times $\frac{n}{k}=1.33$ (see equation (88) for more details on this calculations), resulting in a baud rate of $66.6 \mathrm{GBaud}$. If the dimensionality is increased to 4D then the $\frac{n}{k}$ would drop to $8 / 7=1.14$ resulting in a required baud rate of 57.14 in order to reach a 100 Gbps speed. If the required baud rate is decreased when dimensionality is increased then an improvement in performance is also expected. However, the complexity of the decoding (which is based on calculating the Euclidean distance to all points in the subset) increases exponentially with the dimensionality and number of levels of the parent modulation. Thus, realistically, the complexity of the decoder is too high when the number of dimensions is higher than four or five.

\subsection{The 3D PAM-8 multidimensional set-partitioning modulation}

As explained in the introduction of this chapter the target multidimensional set-partitioning techniques is to increase the distance between the constellation points of the modulation. In more practical terms the goal is to enable $200+$ Gbps optical transmissions employing only commercially available components. At the moment of the writing of this thesis the fastest available DAC on the market has a sample rate of maximum 100 GSamples/s. This means that by using the PAM-4 modulation we can transmit a baud rate of maximum $100 \mathrm{GBaud}$, resulting in a transmission speed of $200 \mathrm{Gbps}$. While it is possible to transmit signals with less than one sample/symbol (thus enabling PAM-4 at speeds exceeding $200 \mathrm{Gbps}$ ) this comes at the cost of severe performance penalties which are too big in the current context. When using a multidimensional set-partitioning modulation we also have to consider the extra baud rate required to accommodate the extra redundancy bits (see example from previous section, last paragraph). Thus, in order to achieve bit rates higher than 200 Gbps the PAM-8 was chosen as the parent modulation. As shown in Table 7 increasing the number of redundancy bits $r$ beyond one provides diminishing returns. To keep implementation complexity low the modulation scheme introduced in this section is limited to only 3D. Taken all the above observations into consideration a 3D PAM-8 set-partitioning scheme with $r=1$ redundancy bits (to which we refer to in this section simply as 3D PAM-8) is proposed for achieving data rates higher than 200 Gbps when employing only commercially available components. The generation of this modulation scheme is shown below.

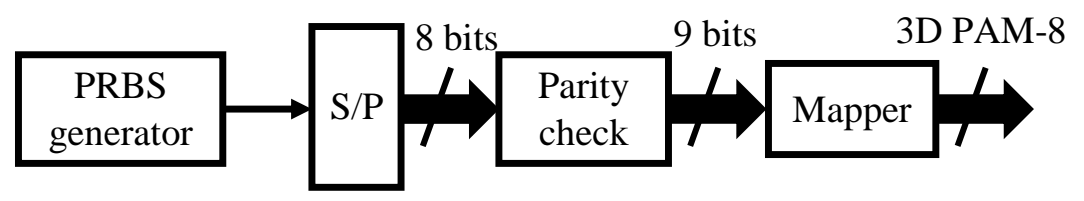

Figure 7.4. 3D PAM-8 set-partitioning modulation generation block diagram 
In classic TCM the transmitted bits are separated in groups. Each group of bits is mapped onto a multidimensional PAM constellation [100]. The full constellation is partitioned into several subsets (power of two number of sets) such that the minimum distance, $d_{\text {min }}$, between the points of one subset is maximized and is larger than the $d_{\min }$ between the points in the full constellation [107], [118]. Some of the bits in one group encode the point within a subset and the others encode the subset number. A convolutional encoder is employed to increase the $d_{\text {min }}$ between the bits encoding the subset number. Soft information and a Viterbi decoder are required at the receiver side in order to recover the original bits. The decoding process loses accuracy in the presence of ISI and when DB is employed [4]. To solve this problem the 3D PAM-8 removes the convolutional encoder and keeps only the set partitioning technique of the TCM, similarly to the approach in [119].

The generation of a 3D PAM-8 symbol is illustrated in Figure 7.4. Firstly, the transmitted bits are organized in groups of 8 . The 3D PAM-8 constellation has a total of 512 points (i.e. the number of levels to the power of number of dimensions), meaning that a maximum of nine bits can be mapped onto this constellation. By using eight bits only 256 points are needed. Thus, the full constellation can be split in two subsets, as shown in Figure 7.3(a) (just 2D for clarity). All of the 256 symbols are mapped to subset 1 . This is done by adding a ninth redundancy bit, as described by the following equation:

$$
b_{9}=\left(b_{3}+b_{6}\right) \% 2
$$

where ' $\%$ ' is the modulo operator. If $b_{1}$ is considered the MSB, then the 3D PAM-8 symbol is obtained by converting the nine bits from binary to an octal base, as described by equation (94):

$$
S_{i}=4 b_{3(i-1)+1}+2 b_{3(i-1)+2}+b_{3(i-1)+3}
$$

If a DB transmission is desired then an extra step is required after the mapper, namely a differential encoder. The bandwidth limited transmission system acts as a $1+1 z^{-1}$ filter, resulting in a received signal with 15 levels. The resulting 3D DB PAM-8 constellation is displayed in Figure 7.3(b) (in 2D only again for clarity). Since only eight out of the nine bits carry information, the baud rate of 3D PAM- 8 must be increased by $9 / 8$ in order to transmit at the same bit rate as the classic PAM-8. If we assume a PAM-8 signal with an amplitude ranging from 0 to 7 then the $d_{\min }=1$. The same holds true for the full 3D PAM-8 constellation. However, due to the encoding, only half of the points (subset 1) are used to map the information bits onto the symbols and thus the $d_{\text {min }}$ is increased from 1 to 1.41 (see Figure 7.3(a)). The same $d_{\min }$ gain ratio (i.e. 1.41) is true for the DB case, where the $d_{\text {min }}$ is raised from 0.5 in the case of DB PAM- 8 to 0.707 for 3D DB PAM-8 (see Figure 7.3(b)). At the receiver side, for each of the 3D PAM-8 symbols the Euclidean distance to each of the points in subset 1 is calculated and the closest constellation point to the received symbol is determined. To recover the transmitted bits this point is converted back from octal to binary. In the case of regular PAM-8 only eight Euclidean distances have to be computed, while for 3D PAM-8 256 are required; thus, the noise tolerance gain comes at the cost of increased 
decoder complexity. In the case of a DB transmission an extra modulo 8 operation is required after the closest constellation point has been identified.

In order to evaluate the capabilities of the 3D PAM-8 scheme a couple of simulations are performed. First, we evaluate the maximum gain, in terms of SNR, that the multidimensional modulation scheme proposed here can provide. For this purpose a transmission where only AWGN is added is simulated. The results of this simulation are shown in Figure 7.5. At the 7\% HD-FEC threshold it can be seen that 3D PAM-8 provides a gain of $1.4 \mathrm{~dB}$ in terms of SNR. The lower the BER the larger the gain gets, with a gain of $1.9 \mathrm{~dB}$ at a BER of $10^{-4}$. Since bandwidth is not simulated for this simulation, DB signaling is not applicable.

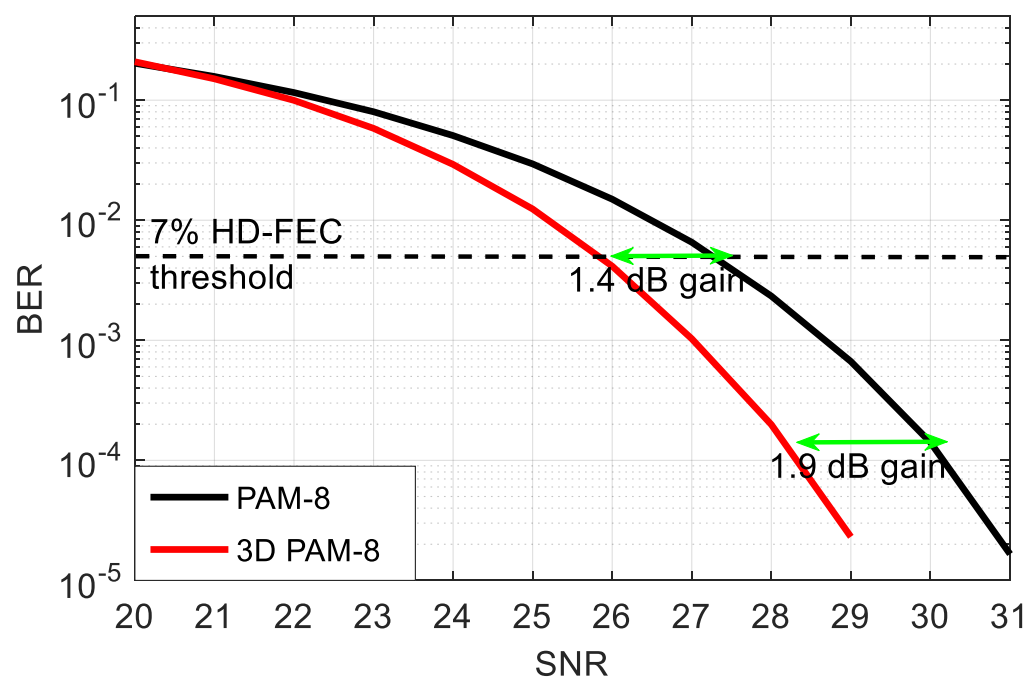

Figure 7.5. Performance comparison between PAM-8 and 3D PAM-8 in the presence of noise and without bandwidth constraints

Next, a more realistic simulation is performed. A 270 Gbps transmission speed is assumed. The 3$\mathrm{dB}$ bandwidths of the transmitter and receiver are set to $80 \mathrm{GHz}$ for a classic non-DB transmission and to $30 \mathrm{GHz}$ when a DB approach is used. The noise introduced by the different electrical components is added on the received photocurrent. The amount of electrical noise is dependent on the desired SNR. The results from this simulation scenario are displayed in Figure 7.6. For the non-DB case a gain of $1.3 \mathrm{~dB}$ in terms of SNR is observed at the FEC threshold. This gain rises to $1.9 \mathrm{~dB}$ at a BER of $10^{-4}$. The gains are almost the same as in the previous simulation, which is to be expected when there is enough bandwidth. For DB this is not the case anymore, thus the performances of both DB PAM-8 and 3D DB PAM-8 drop by almost $3 \mathrm{~dB}$ in comparison with the non-DB scenario. The gain between DB PAM-8 and 3D DB PAM-8 is also decreased, from 1.3 to $1 \mathrm{~dB}$ at the FEC threshold and from 1.9 to $1.1 \mathrm{~dB}$ at $10^{-4}$ BER, as can be observed in Figure 7.6. 


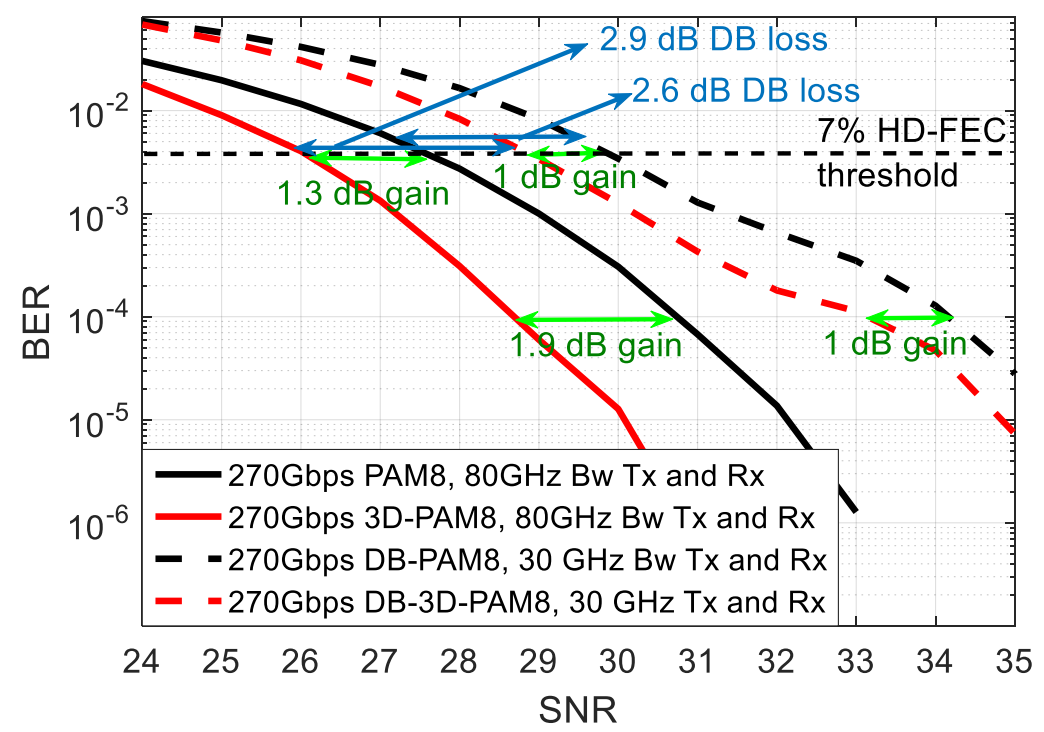

Figure 7.6. Performance comparison between PAM-8 and $3 D$ PAM-8 (continuous lines) and DB PAM-8 and $3 D$ DB PAM-8 (dashed lines)

In order to experimentally test the benefits of 3D PAM-8 a setup made out of only commercially available components is employed. The block diagram of the experimental setup can be seen in Figure 7.7. The first steps are to generate a PRBS in MATLAB, to calculate the redundancy bit, to perform Gray coding and to map the resulting bits onto the PAM-8 or 3D PAM-8 constellation (see previous section for a more detailed description of the 3D PAM-8 mapping). The last step in the digital domain at the transmitter side is to resample the data. After resampling the analog signal is generated with the help of an AWG with a 3-dB bandwidth of $32 \mathrm{GHz}$. The signal at the output of the AWG has a PtP amplitude of $200 \mathrm{mV}$, which is not enough to drive the modulator. Thus, the AWG is followed by a 6-dB attenuator and a 24-dB gain driver (for a total amplification of 18 $\mathrm{dB}$ ). The signal is converted from electrical to optical by a $30 \mathrm{GHz}$ bandwidth single-drive MZM. The optical signal to the MZM is provided by a CW laser at a wavelength of $1551.6 \mathrm{~nm}$ and an output power of $13 \mathrm{dBm}$. The optical signal is transmitted BTB and converted back to the electrical domain by a PD receiver (the input power to the PD is $7 \mathrm{dBm}$ ). Received data is sampled at a rate of 160 GSamples/sec by a $63 \mathrm{GHz}$ Bandwidth real-time oscilloscope. The offline data is resampled to two samples/symbol and then timing recovery is performed. To mitigate the influence of the limited bandwidth (the $3-\mathrm{dB}$ bandwidth of the whole system is only $13 \mathrm{~dB}$ ) and the effects of nonlinearities a Volterra filter is employed [120]. This filter has 121 linear taps, 36 taps for the second order kernel and 84 taps for the third order kernel. If 3D PAM-8 is transmitted then a Euclidean distance decoder is added to recover the original transmitted data (more details on decoding in the previous section). In the end Gray decoding is performed and the BER is calculated in order to evaluate the performance of the transmission. 


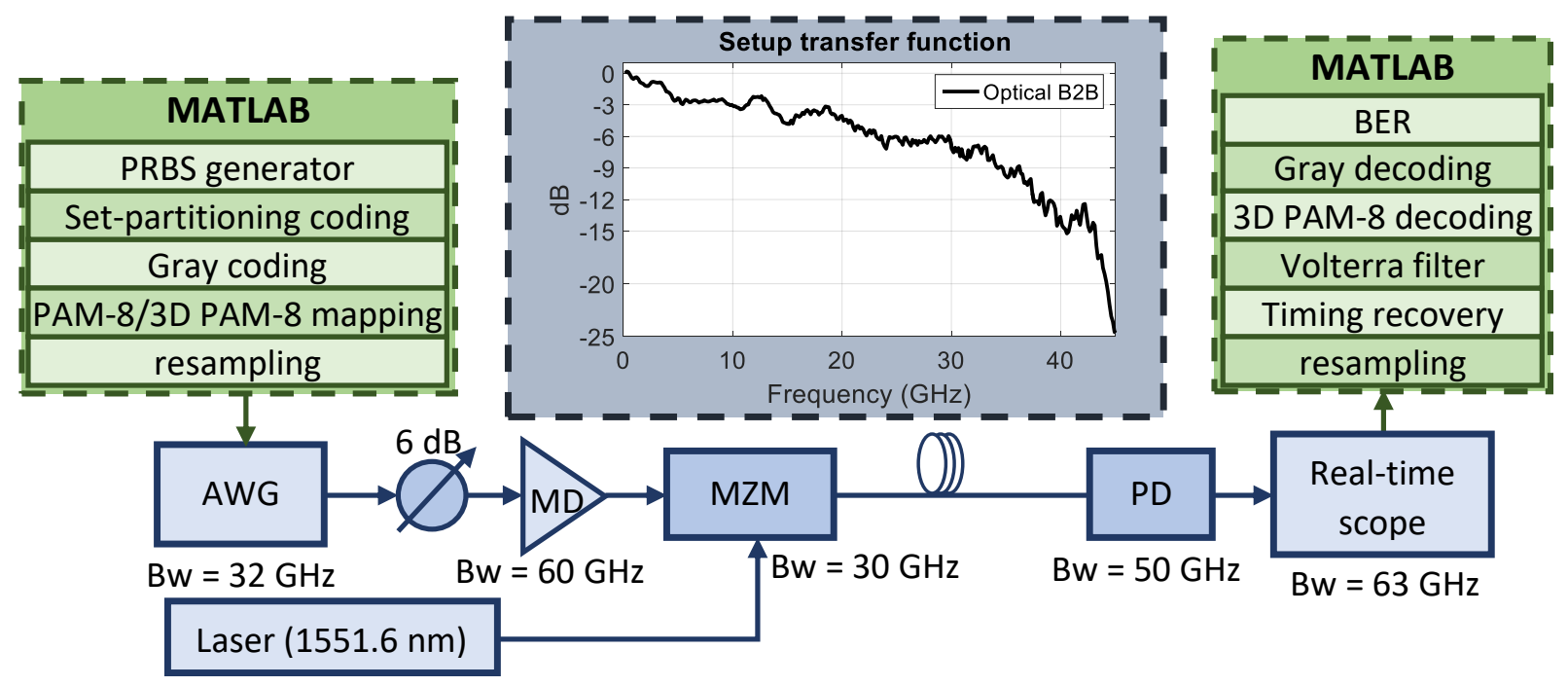

Figure 7.7. 3D PAM-8 experimental setup and system transfer function

The performance of PAM-8 and 3D PAM-8 is tested at bit rates ranging from 56 up to 240 Gbps. The results of this investigation are displayed in Figure 7.7. At lower rates the non-DB versions of PAM-8 and 3D PAM-8 are employed. At 56 Gbps the multidimensional modulation lowers the BER by roughly 10 times. At this transmission rate the transfer function of the whole system (see inset to Figure 7.7) is not limiting too much the bandwidth of the signal, meaning that the results here can be compared with the simulation results from Figure 7.6 (continuous line curves); when looking at BERs around $10^{-4}$ in simulation, it can be observed that the BER is lowered by more than 10 times when the 3D PAM-8 is used, which is very close to the reduction seen in the experimental results. As the bit rate is increased the BER gain of 3D PAM-8 becomes smaller and smaller. At a rate of $180 \mathrm{Gbps}$ the 3D PAM-8 scheme can no longer outperform the classic PAM8. As the transmission rate is increased, the effects of ISI become more and more severe, overshadowing the influence of noise. Since 3D PAM-8 improves noise tolerance but is more sensitive to bandwidth limitations (due to it requiring higher baud rates than PAM-8 in order to achieve the same bit rate), it makes sense that its performance degrades faster than that of PAM8. At bit rates higher than $180 \mathrm{Gbps}$ the non-DB approach fails to provide BERs below the FEC threshold, for both PAM-8 and its multidimensional counterpart.

In order to achieve a net rate of $200 \mathrm{Gbps}$ or more, a transmission rate of at least $214 \mathrm{Gbps}$ is necessary, when accounting for the FEC overhead. The currently commercially available components limit the usable spectrum to below $45 \mathrm{GHz}$, as can be seen in the inset to Figure 7.7, where the spectral power for frequencies above $40-45 \mathrm{GHz}$ is severely attenuated. As shown in the previous paragraph classic PAM-8 cannot go beyond $180 \mathrm{Gbps}$ in these conditions, thus a technique such as DB signaling is required to increase the spectral efficiency of the signal. The experimental results for DB PAM-8 and 3D DB PAM-8 are shown in Figure 7.8 (the dashed line curves), for bit rates ranging from $180 \mathrm{Gbps}$ and up to $240 \mathrm{Gbps}$. If the performance of DB at 180 Gbps is compared with that of non-DB at the same bit rate it can be observed that DB achieves lower BER. In fact, when targeting the same BER, the DB approach enables BTB transmissions 
that are around 40 to $60 \mathrm{Gbps}$ faster. By using DB signaling the spectral efficiency of the signal is greatly improved at the cost of noise tolerance (the same PtP voltage now contains 15 levels instead of 8). Thus, noise becomes again the major impediment and as a result the 3D DB PAM-8 outperforms the DB PAM-8, although the maximum BER gain is decreased when compared with the maximum gain in the non-DB case. For all the rates between 180 and 240 Gbps the multidimensional modulation outperforms DB PAM-8, lowering the BER by about three times. When comparing with the simulation results (Fig.4, dashed lines) similar gains can be observed, where 3D DB PAM-8 lowers the BER by roughly 3-4 times for BERs below the FEC threshold.

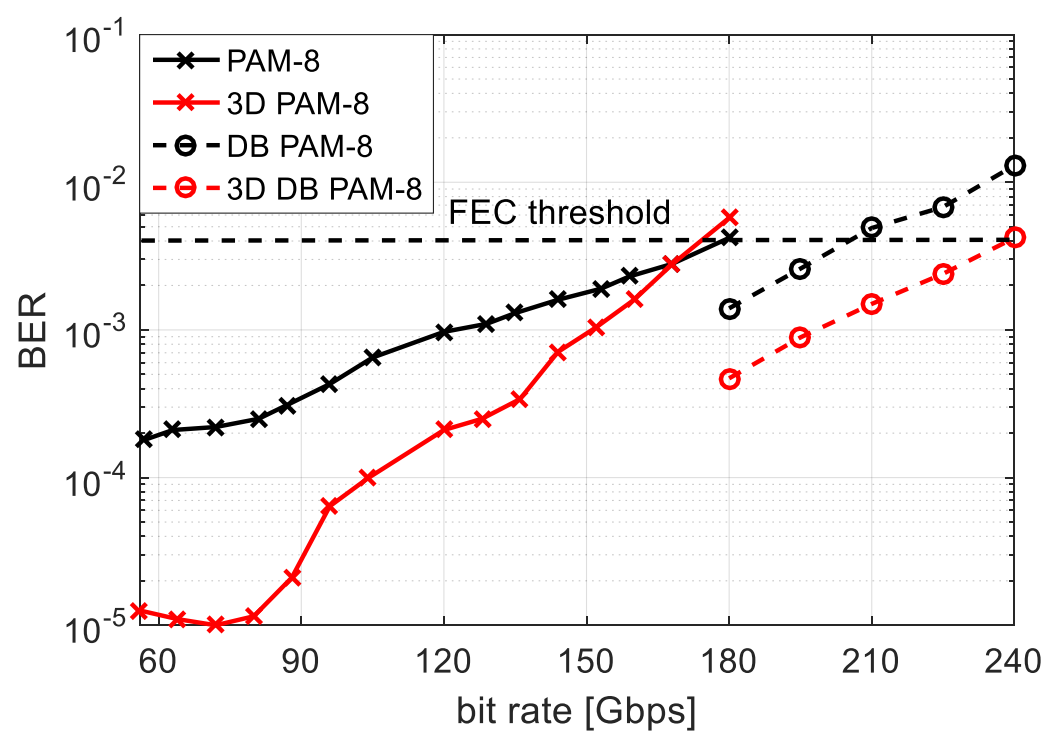

Figure 7.8. BER performance comparison between PAM-8 and $3 D$ PAM-8 for bit rates from 56 to 180 Gbps (continuous lines) and between DB PAM-8 and $3 D$ DB PAM-8 for bit rates between 180 and 240 Gbps (dashed lines)

The DB PAM-8 modulation can achieve BERs lower than the 4×10-3 FEC threshold for transmission rates of up to $205 \mathrm{Gbps}$. This is below the $214 \mathrm{Gbps}$ required for a $200 \mathrm{G}$ singlelambda data rate and thus it can be concluded that PAM-8, be it non-DB or DB, cannot be employed in such high-speed optical transmission. On the other hand, with 3D DB PAM-8, bit rates of up to $240 \mathrm{Gbps}$ are achieved at a BER below the FEC threshold, making it a viable option for the next generation of high speed intra- and inter-datacenter networks. The experimental setup used here limits the 3D PAM-8 transmission to a maximum of $240 \mathrm{Gbps}$ due to the limited sampling rate of the AWG (90 GSamples/sec).

When aiming for $200 \mathrm{G}$ or more single-lambda IM-DD transmissions the current commercially available components introduce severe bandwidth limitations, making the use of DB signaling a must. The SNR of such transmission systems is also a big limiting factor. TCM is usually employed to improve the noise tolerance, however it does not work properly in conjunction with DB. In this thesis a simplified, less complex form of TCM, referred to here as 3D PAM-8, is proposed. This scheme improves SNR sensitivity by $1.4 \mathrm{~dB}$ at the $7 \%$ HD-FEC threshold in transmission systems 
with no significant ISI. In both simulations and experiments it is demonstrated that 3D PAM-8 can reduce the BER (when compared with PAM-8) by more than 10 times in the non-DB case and by roughly three times when DB is employed. In this section it has been proven also that, when using only commercially available components, 3D PAM- 8 enables transmission rates of up $240 \mathrm{Gbps}$ at BERs below the FEC limit, while classic PAM-8 is unable to achieve such high data rates. 


\section{Chapter 8 Conclusions}

In this Ph.D. thesis the most common signal processing techniques employed in state-of-the-art non-coherent optical transmission systems are investigated. On top, some novel IM-DD DSP approaches are considered and their advantages and drawbacks are analyzed. While contemporary optical IM-DD systems have to deal with increasing amounts of CD and bandwidth limitations as the transmission rates also increase, it is shown in this that DSP can be used to greatly mitigate their effects and some novel approaches are suggested in order to keep optical non-coherent system competitive in the race with coherent solutions for the short-reach market.

The first two chapters introduce the challenges facing modern IM-DD optical system and give an overview of such system. The different components employed in the experimental setups used to test the different DSP algorithm are presented. Because of the difficulties the author encountered when looking for written instructions on building and operating an optical experimental setup, the aim of chapter 2 is to enable the reader to easily build an experimental setup to test his/her algorithms and to give an overview of how to use each component.

The investigation into DSP techniques for multilevel intensity modulation starts with chapter 3, where the two most widespread algorithms, the FFE and the MLSE, are analyzed. It is shown that the FFE is a very strong equalizer capable of compensating for large amounts of bandwidth limitations but is very sensitive to CD. On the other hand, MLSE can compensate for the effects of both bandwidth limitations and CD very well, however, its implementation complexity is higher than that of FFE and linear improvements in its performance come at the cost of exponential increases in its complexity. At least one of these two DSP algorithms are present in most noncoherent optical systems available today.

While FFE and MLSE can be employed to effectively equalize the received signal and to combat the CD from the link, respectively, they cannot improve the noise tolerance of the transmission system. The main factor determining the sensitivity to noise of a transmission is the modulation. Since in this thesis only the intensity modulation is investigated, the sensitivity to noise is determined mainly by the number of amplitude levels of the modulation (the higher the number of levels, the less noise can be tolerated). While in a pure electrical transmission the optimal constellation shaping would be equidistant and equiprobable modulation levels, in optical noncoherent transmissions (which combine electrical and optical) this is no longer the case. In the fourth chapter the optimum geometrical and probabilistic shaping of PAM schemes is investigated 
and it is shown experimentally that the noise tolerance can be greatly increased by employing the constellation shaping techniques presented in this chapter.

As the demand for bit rate per wavelength has increased in the last few years to 100 Gbps and beyond, so have the bandwidth requirements on components. However, the bandwidth of the commercially available components has not kept up the pace resulting in an increase in the bandwidth limitations in modern IM-DD optical systems. In chapter 5 a DSP technique meant to combat the effects of bandwidth restrictions (i.e. the partial response signaling) is investigated in detail. It is proven here that the most common form of PRS, namely the DB signaling, is an essential technique for high speed, low-bandwidth systems, providing significant received power sensitivity gains for heavily bandwidth limited systems. More advanced forms of PRS can be also customized to combat the influence of CD. In this thesis, such PRS is shown to be capable of extending the range of a non-coherent optical transmission by up to three times. In the same chapter the concept of DPE is also introduced. This technique is shown to be capable of mitigating the effects of a lack of bandwidth and/or reducing the complexity of the receiver DSP (i.e. the complexity of the FFE).

In the high-speed IM-DD optical transmission experiments performed during this Ph.D. research, it is common to see a so-called error floor (i.e. the BER does not go below a certain value, no matter the input power to the PD). The error is caused mainly by noise. In chapter 6 another technique which combat the effects of noise is presented, the TCM. The TCM incorporates FEC elements together with set-partitioning techniques and multidimensional modulation in order to provide a modulation that has increased distance between constellation points. It is demonstrated in both simulation and experimentally that TCM can drastically improve the sensitivity of a system in the right scenarios and as a result can also enable longer transmissions in the O-band. The drawbacks of this technique are its sensitivity to bandwidth limitations (which means it cannot be used together with DB signaling) and its high implementation complexity, limiting the scenarios in which TCM is usable. In chapter 6 a modified version of TCM is proposed, the multidimensional set-partitioning modulation. In this version the FEC components are removed, while the setpartitioning and multidimensional modulation are kept. By doing this, although the maximum gain achievable is reduced, the modulation is no longer highly sensitive to bandwidth restrictions and the complexity is also greatly reduced. This modulation scheme can now be combined with DB signaling, enabling transmission rates higher than $200 \mathrm{Gbps}$ and $800 \mathrm{Gbps}$ to 1 Tbps WDM systems, thus proving that IM-DD solutions are still viable for next generation of short-reach optical transceivers.

While the feasibility of optical transmissions faster than $200 \mathrm{Gbps}$ per lambda has been proven in chapter 7, the achieved BERs are quite high and close to the FEC threshold, as can be observed in Figure 7.8. Although in the laboratory environment BERs below the FEC threshold are achieved, in real-life practical implementation there are additional things to consider (such as price and power consumption of the components used or additional connectors) which will negatively influence the overall performance of the optical system. This means that, from a product point of 
view, the experimental BER should be considerably lower than the FEC threshold. As a result of this, the main goal going forward is to further improve the performance of the high speed optical non-coherent transmission. The rough target is to achieve a BER below $10^{-5}$ for transmission rates larger than 200 Gbps. One investigation direction is to improve the ISI tolerance of TCM. In chapter 6 it has been shown that TCM can greatly improve noise tolerance but is susceptible to the effects of bandwidth limitations. If the negative influence of ISI on TCM could be minimized then this modulation technique could significantly reduce the BER floor in $800 \mathrm{Gbps}$ WDM optical systems. Since in IM-DD transmissions power consumption is a major concern, reducing the complexity of the DSP is also of great interest. The Volterra filter, which combines the linear FFE with a second and third order nonlinear equalizer, requires a very high number of taps to function properly in the context of very high-speed optical transmissions. Another future research direction is to investigate the training of the Volterra filter and determine the most significant taps. This would help 'trim' the Volterra equalizer by removing linear and nonlinear taps that don't contribute in any significant way, thus helping reduce DSP complexity and, consequentially, power consumption. 


\section{Annex A. Publications}

Chapter 3 and Chapter 4 - The experimental results from studying the MLSE algorithm where published in the following journal paper:

- C. Prodaniuc, N. Stojanovic, and Z. Qiang, "Performance of Fractionally Spaced MLSE in OOK and PAM-4 Bandwidth Limited Optical Systems," in TELFOR Journal, vol. 7, no. 2, pp. 74-79, 2016

The concept of geometrical constellation shaping was proposed due to its potential for mitigating the negative influence of fiber dispersion. The experimental demonstration was published in:

- C. Prodaniuc, N. Stojanovic, F. Karinou, G. Goeger, Z. Qiang, and R. Llorente, "56 Gb/s, PAM-4 Transmission Over 25 km, Using IQ Modulator and Unequally Spaced Levels," in Conference of Laser Science (CLEO) proceedings, 2015

Chapter 5 - The first three sections of this chapter investigate the performance of PAM schemes in high-bitrate transmission scenarios. DB PAM-4 modulation was proposed as an interesting option for reducing bandwidth requirements. This work was published in:

- C. Prodaniuc, N. Stojanovic, F. Karinou, Z. Qiang, Thomas Dippon, and Roberto Llorente, "PAM-n Solutions for Low-Cost Implementations of 100 Gbps/Lambda Transmissions," in European Conference on Optical Communications (ECOC) proceedings, 2016

The fourth section investigates another technique for mitigating the influence of ISI caused by limited bandwidth. The findings were published in:

- C. Prodaniuc, N. Stojanovic, Z. Qiang, and R. Llorente, "Two-tap Digital Pre-Emphasis for Low-Bandwidth 112 Gbps, PAM-4 Transmissions," in IEEE Photonics Conference (IPC) proceedings, 2016

The last section of this chapter proposes a novel approach based on using PRS to mitigate the impact of CD. This technique was reported in the following journal paper:

- C. Prodaniuc, N. Stojanovic, F. Karinou, and R. Llorente, "Partial response signaling for improved chromatic dispersion tolerance in intensity modulation optical transmissions," in OSA Optics Express, vol. 26, no. 3, pp. 3013-3019, 2018

Chapter 6 - this chapter is dedicated to the investigation of TCM schemes. The generalized TCM scheme is introduced and then the performance is analyzed, both in simulation and experimentally. 
The findings from the analysis were published first in a conference paper. A complete in-depth analysis was presented in a journal paper:

- C. Prodaniuc, N. Stojanovic, Z. Qiang, F. Karinou, T. Lee, K. Engenhardt, and R. Llorente, "Experimental Demonstration of 56 Gb/s 4D-PAM-5 Trellis Coded Modulation for $400 G$ WDM Metro-Access Networks," in the Optical Fiber Communication Conference and Exhibition (OFC) proceedings, 2016

- C. Prodaniuc, N. Stojanovic, F. Karinou, Z. Qiang, and R. Llorente, "Performance Comparison between 4D Trellis Coded Modulation and PAM-4 for Low-cost 400 Gbps WDM Optical Networks," in IEEE/OSA Journal of Lightwave Technology, vol. 34, no. 22, pp. $5308-5316,2016$

Chapter 7 - A simplified version of TCM using multidimensional set-partitioning techniques, which does not employ any FEC elements, is proposed in this chapter. Its performance and advantages over TCM are reported in the following article:

- C. Prodaniuc, N. Stojanovic, C. Xie, Z. Liang, J. Wei, and R. Llorente, "3-Dimensional PAM8 Modulation for 200 Gbps/lambda Optical Systems," in Elsevier Optics Communications, 2018 


\section{References}

[1] F. O. Components, "What is the Meaning of $100 \mathrm{G}$ Channels Networks to Service Providers," [Online]. Available: http://www.fiber-optic-components.com/what-is-the-meaning-of-100gchannels-networks-to-service-providers.html.

[2] CISCO, "The zettabyte era: trends and analysis," White paper, July 2017.

[3] L. -. M. Research, "Optical connectivity cloud sales forecast bright," [Online]. Available: https://www.cablinginstall.com/articles/2018/05/cloud-sales-Iw.html.

[4] C. Prodaniuc, N. Stojanovic, F. Karinou, Z. Qiang and R. Llorente, "Performance comparison between 4D trellis coded modulation and PAM-4 for low-cost 400 Gbps WDM optical networks," J. of Light. Tech. , vol. 34, no. 22, pp. 5308-5316, 2016.

[5] L. Zhang and N. S. C. P. a. C. X. J. Wei, "Beyond 200-Gb/s DMT transmission over 2-km SMF based on a low-cost architecture with single-wavelength, single-DAC/ADC and single-PD," in ECOC We1H.1, Rome, 2018.

[6] [Online]. Available: http://100glambda.com/specifications/send/2-specifications/1-400g-fr4technical-spec-d1p0.

[7] K. Grobe and M. Eiselt, Wavelength division multiplexing: A practical engineering guide, Wiley Telecom, 2014.

[8] K. Tamura, D. Lewis and P.Jones, "25G SMF Optics for Next Generation Enterprise / Campus / Data Centre Applications," [Online]. Available: http://www.ieee802.org/3/ad_hoc/ngrates/public/15_09/tamura_nge_01_0915.pdf.

[9] S. Kanazawa, T. Fujisawa, K. Takahata, Y. Nakanishi, H. Yamazaki, Y. Ueda, W. Kobayashi, Y. Muramoto, H. Ishii and H. Sanjoh, "56-Gbaud 4-PAM (112-Gbit/s) operation of flip-chip interconnection lumped-electrode EADFB laser module for equalizer-free transmission," in OFC, W4J.1, Anaheim, 2016.

[10] C. Chen, X. Tang and Z. Zhang, "Transmission of 56-Gb/s PAM-4 over 26-km single mde fiber using maximum likelihood sequence estimation," in OFC, Th4A.5, Valencia, 2015.

[11] J. Lee, P. Dong, N. Kaneda and Y.-K. Chen, "Discrete multi-tone transmission for short-reach optical connections," in OFC, Th1G.1., Anaheim, 2016. 
[12] K. Zhong, X. Zhou, T. Gui, Q. Sui, Y. Gao, L. Wang, A. P. T. Lau and C. Lu, "Advanced modulation formats for 100Gb/s/lambda short reach applications," in ICOCN, Nanjing, China, 2015.

[13] F. Karinou, N. Stojanovic, C. Prodaniuc, M. Agustin, J. Kropp and N. N. Ledentsov, "Solutions for 100/400-Gb/s Ethernet systems based on multimode photonic technologies," J. of Light. Tech., vol. 35, no. 15, pp. 3214 - 3222, 2017.

[14] C. Prodaniuc, N. Stojanovic, Z. Qiang and R. Llorente, "Two-tap digital pre-emphasis for lowbandwidth 112 Gbps, PAM-4 transmissions," in IPC, MB4.4, Waikoloa, Hawaii, 2016.

[15] N. Stojanovic, Z. Qiang, C. Prodaniuc and F. Karinou, "Performance and DSP complexity evaluation of a 112-Gbit/s PAM-4 transceiver employing a 25-GHz TOSA and ROSA," in ECOC, Tu.3.4.5, Valencia, 2015.

[16] MathWorks, "MATLAB homepage," [Online]. Available: https://www.mathworks.com/products/matlab.html.

[17] CCITT, "ITU-T recommendation 0.150," [Online]. Available: http://www.itu.int/rec/T-REC-O.150199210-S.

[18] SHF. [Online]. Available: https://www.shf-communication.com/wpcontent/uploads/datasheets/datasheet_shf_12103_a.pdf.

[19] J. G. Proakis, Digital communications, The McGraw-Hill Companies, Inc., 2001.

[20] K. Technologies, "M8196A 92 GSa/s Arbitrary Waveform Generator," [Online]. Available: https://www.keysight.com/en/pd-2583267-pn-M8196A/92-gsa-s-arbitrary-waveformgenerators? $\mathrm{nid}=-33319.1139580 \& \mathrm{cc}=\mathrm{US} \& \mid \mathrm{c}=\mathrm{e}$. .

[21] H. Zwickel, S. Wolf, Y. Kutuvantavida, C. Kieninger, M. Lauermann, W. Freude and C. Koos, "120 Gbit/s PAM-4 signaling using a silicon-organic hybrid (SOH) Mach-zehnder modulator," in ECOC, Duesseldorf, 2016.

[22] Q. Zhang, N. Stojanovic, T. Zuo, L. Zhang, C. Prodaniuc, F. Karinou, C. Xie and E. Zhou, "Single-lane $180 \mathrm{~Gb} / \mathrm{s}$ SSB-duobinary-PAM-4 signal transmission over $13 \mathrm{~km} \mathrm{SSMF,"} \mathrm{in} \mathrm{OFC,} \mathrm{Tu2D.2,} \mathrm{Los}$ Angeles, 2017.

[23] F. Karinou, N. Stojanovic and C. Prodaniuc, " $56 \mathrm{~Gb} / \mathrm{s} 20-\mathrm{km}$ transmission of PAM-4 signal employing an EML in C-band without in-line chromatic dispersion compensation," in ECOC, M.2.C, Duesseldorf, 2016.

[24] D. Che, F. Yuan, Q. Hu and W. Shieh, "Complex modulation of directly modulated lasers for medium reach optical communications," in OFC, Tu2A.5, Anaheim, 2016.

[25] J. Zhang, T. Ye, X. Yi, C. Yu and K. Qiu, "An efficient hybrid equalizer for 50 Gbps PAM-4 singal transmission over $50 \mathrm{~km}$ SSMF in a 10-GHz DML-based IM/DD system," in CLEO, SF1L.1, San Jose, 2017. 
[26] J. Lavrencik, S. Varughese, V. A. Thomas, G. Landry, Y. Sun, R. Shubochkin, K. Balemarthy, J. Tatum and S. E. Ralph, "4-lambda x 100Gbps VCSEL PAM-4 transmission over 105m of wide band multimode fiber," in OFC, Tu2B.6, Los Angeles, 2017.

[27] N. Eiselt, H. Griesser, J. Wei, R. Hohenleitner, A. Dochhan, M. Ortsiefer, M. H. Eiselt, C. Neumeyr, J. J. V. Olmos and I. T. Monroy, "Experimental demonstration of $84 \mathrm{~Gb} / \mathrm{s}$ PAM-4 over up to $1.6 \mathrm{~km}$ SSMF using a 20-GHz VCSEL at 1525 nm," J. of Light. Tech. , vol. 35, no. 8, pp. 1342-1349, 2017.

[28] F. Karinou, N. Stojanovic, A. Daly, C. Neumeyr and M. Ortsiefer, "1.55- $\mu$ m long -wavelength VCSEL-based optical interconnects for short-reach networks," J. of Light. Tech., vol. 34, no. 12, pp. 2897-2904, 2016.

[29] L. N. Binh, "Optical fiber communication systems with MATLAB and Simulink models", London: CRC Press, Taylor\&Francis Group, 2015.

[30] X. Lin, Electroabsorption modulators used for all-optical signal processing and labelling, $\mathrm{PhD}$ Thesis: Danish Technical University, 2004.

[31] VPIphotonics. [Online]. Available: http://vpiphotonics.com/Tools/ComponentDesign/.

[32] M. Suzuki, Y. Noda, H. Tanaka, S. Akiba, Y. Kushiro and H. Isshiki, "Monolithic integration of InGaAsP/InP distributed feedback laser and electroabsorption modulator by vapor phase epitaxy," Journal of Lightwave Technology, Vols. LT-5, no. 9, pp. 1277-1285, 1987.

[33] W. Franz, "Einfluß eines elektrischen Feldes auf eine optische Absorptionskante," Z. Naturforschung, vol. 13a, p. 484-489, 1958.

[34] L. V. Keldysh, "Behavior of non-metallic crystals in strong electric fields," Soviet Physics JETP, vol. 6, no. 33, pp. 763-770, 1958.

[35] Y. Jiang, High-speed electronic signal processing for pre-compensation in optical communications, PhD thesis, Queen's University, Ontarion, Canada, 2011.

[36] G. P. Agrawal, "Lightwave technology - telecommunications systems", New Jersey: John Wiley \& Sons, Inc., 2005.

[37] M. Morant, A. Macho and R. Llorente, "On the suitability of multicore fiber for LTE-advanced MIMO optical fronthaul systems," Journal of Lightwave Technology, vol. 34, no. 2, pp. 676-682, 2016.

[38] R. Llorente, A. Macho, D. Garcia-Rodriguez, A. Zainullin, M. Morant and J. L. Corral, "Towards multidimensional multiplexing in multicore fiber optical data links," in International Conference on Transparent Optical Networks, Trento, Italy, 2016.

[39] R. Paschotta, "Polarization Mode Dispersion," RP Photonics Consulting GmbH, [Online]. Available: https://www.rp-photonics.com/polarization_mode_dispersion.html. 
[40] S. Ten and M. Edwards, "An introduction to the fundamentals of PMD in fibers," Corning Incorporated, New York, 2006.

[41] S. P. Singh and N. Singh, "Nonlinear effects in optical fibers: origin, management and applications," Progress in Electromagnetic Research, vol. 73, pp. 249-275, 2007.

[42] D. Felice, "A study of the nonlinear Schrodinger equation for optical fibers," 2016.

[43] C. Prodaniuc, N. Stojanovic, Z. Qiang, F. Karinou, T. Lee, K. Engenhardt and R. Llorente, "Experimental demonstration of $56 \mathrm{~Gb} / \mathrm{s}$ 4D-PAM-5 trellis coded modulation for 400G WDM metro-access networks," in OFC, Anaheim, 2016.

[44] I. 61280-2-9:2009, "Fibre optic communication subsystem test procedures - Part 2-9: Digital systems - Optical signal-to-noise ratio measurement for dense wavelength-division multiplexed systems," International standard, 2009.

[45] R. W. World, "What is OSNR-Optical Signal to Noise Ratio," [Online]. Available: http://www.rfwireless-world.com/Terminology/What-is-OSNR.html.

[46] S. M. Abuzariba, Theoretical calculation of system performance of fiber optic network with chromatic dispersion, polarization mode dispersion, polarization dpendant loss, and amplifier spontaneous emission noise, University of Canada, Ottawa, 2010.

[47] H. A. Haus, Electromagnetic noise and quantum optical measurements, New York: Springer, 2000.

[48] S. Bottacchi, Noise and signal interference in optical fiber transmission systems, Singapore: Wiley, 2008.

[49] O. Ozolins, X. Pang, M. I. Olmedo, A. Kakkar, A. Udalcovs, S. Gaiarin, J. R. Navarro, K. M. Engenhardt, T. Asyngier, R. Schatz, J. Li, F. Nordwall, U. Westergren, D. Zibar, S. Popov and G. Jacobsen, "100 GHz externally modulated laser for optical interconnects," J. of Light. Tech., vol. 35, no. 6, pp. 1174-1179, 2017.

[50] Finisar, "200G/100G Tunable C-Band CFP2-ACO Coherent Optical Transceiver," [Online]. Available: https://www.finisar.com/optical-transceivers/ftlc3322×3nl.

[51] K. H. Mueller and M. Müller, "Timing recovery in digital synchronous data receivers," IEEE Trans. Commun., vol. 24, no. 5, p. 516, 1976.

[52] F. M. Gardner, "A BPSQ/OPSK timing-error detector for sampled receivers," IEEE Trans. on Communications, Vols. COM-34, no. 5, pp. 423-429, 1986.

[53] H. Meyr, M. Moeneclaey and S. A. Fechtel, Digital communication receivers, Canada: John Wiley \& Sons, Inc., 1998.

[54] R. E. Best, Phased-locked loops, McGraw-Hill, 1999. 
[55] B. P. Lathi and R. A. Green, Essentials of digital signal procesing, New York: Cambridge University Press, 2014.

[56] A. Beasley and A. Cole-Rhodes, "A blind decision feedback equalizer for QAM signals based on the constant modulus algorithm," in MILCOM 2006 - 2006 IEEE Military Communications conference, 2006.

[57] A. J. Viterbi, "Error bounds for convolutional codes and an asymptotically optimum decoding algorithm," IEEE Trans. Information Theory, Vols. IT-13, pp. 260-269, Apr. 1957.

[58] H. Kobayashi, "Correlative level coding and maximum likelihood decoding," IEEE Trans. Information Theory, Vols. IT-17, pp. 64-74, Sept. 1971.

[59] G. D. Forney, "Maximum likelihood sequence estimation of digital sequences in the presence of intersymbol interference," IEEE Trans. Information Theory, Vols. IT-18, pp. 363-378, May 1972.

[60] J. F. Hayes, "The Viterbi algorithm applied to digital data transmission," IEEE Commun. Mag. , vol. 13, no. 2, pp. 15-20, Mar. 1975.

[61] N. Stojanovic, Z. Qiang and C. Prodaniuc, "Fractionally spaced MLSE in bandlimited optical systems using intensity modulation and direct detection," in TELFOR, Belgrade, 2014.

[62] "IEEE P802.3ba 40Gb/s and 100Gb/s Ethernet Task Force," [Online]. Available: http://www.ieee802.org/3/ba/.

[63] J. Lee, N. Kaneda, T. Pfau, A. Konczykowska, F. Jorge, J.-Y. Dupuy and Y.-K. Chen, "Serial 103.125$\mathrm{Gb} / \mathrm{s}$ transmission over $1 \mathrm{~km}$ SSMF for low-cost, short-reach optical interconnects," in OFC, Th5A.5, 2014.

[64] W. Hartmann, M. Lauermann, S. Wolf, H. Zwickel, Y. Kutuvantavida, J. Luo, A. K.-Y. Jen, W. Freude and C. Koos, "100 Gbit/s OOK using a silicon-organic hybrid (SOH) modulator," in ECOC, PDP1.4, Valencia, 2015.

[65] X. Chen, S. Chandrasekhar, S. Randel, G. Raybon, A. Adamiecki, P. Pupalaikis and P. J. Winzer, "Allelectronic $100-\mathrm{GHz}$ bandwidth digital-to-analog converter generating PAM signals up to 190 Gbaud," Journal of Lightwave Tech. , vol. 35, no. 3, pp. 411-417, Feb. 2017.

[66] T. K. Chan and W. I. Way, "112 Gb/s PAM4 transmission over 40km SSMF using 1.3 um gainclamped semiconductor optical amplifier," in OFC, Th3A.4., San Jose, 2015.

[67] S. Kanazawa, H. Yamazaki and Y. Nakanishi, "Transmission of 214-Gbit/s 4-PAM signal using an ultra-broadband lumped-electrode EADFB laser module," in OFC, Th5B.3, Anaheim, 2016.

[68] "http://www.ieee802.org/3/bs/public/index.html," [Online].

[69] A. Dochhan, M. O. Al-Dwairi and W. Rosenkranz, "Optimization of cost effiecient multilevel-ASK modulation formats under the constraint of chromatic dispersion," in OFC/NFOEC, EMJ7 , 2010. 
[70] L. F. Suhr, J. J. V. Olmos, B. Mao, X. Xu, G. N. Liu and I. T. Monroy, "112-Gbit/s x 4-lane duobinary4-PAM for 400GBase," in ECOC, Tu.4.3.2, Cannes, France, 2014.

[71] T. A. Eriksson, M. Chagnon, F. Buchali, K. Schuh, S. T. Brink and L. Schmalen, "56 Gbaud probabilistically shaped PAM8 for data center interconnects," in ECOC, Tu.2.D.4, Gothenburg, 2017.

[72] P. Schulte and G. Böcherer, "Constant composition distribution matching," IEEE Trans. Inf. Theory, vol. 38, no. 1, pp. 430-434, 2016.

[73] T. V. Ramabadran, "A coding scheme fro m-out-of-n codes," IEEE Trans. Comunn., vol. 38, no. 8, pp. 1156-1163, 1990.

[74] G. Böcherer, F. Steiner and P. Schulte, "Fast probabilistic shaping implementation for long-haul fiber-optic communication systems," in ECOC, Tu.2.D.3, Gothenburg, 2017.

[75] T. Yoshida, M. Karlsson and E. Agrell, "Short-block-length shaping by simple mark ratio controllers for granular and wide-range spectral efficiencies," in ECOC, Tu.2.D.2, Gothenburg, 2017.

[76] F. Buchali, G. Bocherer, W. Idler, L. Schmalen, P. Schulte and F. Steiner, "Experimental demonstration of capacity increase and rate-adaptation by probabilistically shaped 64-QAM," in ECOC, PDP3.4, Valencia, 2015.

[77] R.-J. Essiambre and R. W. Tkach, "Capacity trends and limits of optical communication networks," Proceedings of the IEEE, vol. 100, no. 5, pp. 1035-1055, 2012.

[78] J. Zhou, C. Yu, M. Gurusamy and H. Kim, "25-Gb/s OOK and 4-PAM transmission over >35-km SSMF using directly modulated 1.5-um VCSEL," in OFC, Th1G.7, Anaheim, 2016.

[79] K. Szczerba, M. Karlsson and A. L. P. A. Andrekson, "Intersymbol interference penalties for OOK and 4-PAM in short-range optical communications," in OFC, OW4A.3, 2013.

[80] Z. Liu, T. Richter, C. Schubert, D. J. Richardson and R. Slavik, "Practical considerations on discrete multi-tone transmission for cost-effective access networks," in OFC, M3J.4, 2015.

[81] S. Walklin and J. Conradi, "Multilevel signaling for increasing the reach of $10 \mathrm{~Gb} / \mathrm{s}$ lightwave systems," Journal of Lightwave Technology, vol. 17, no. 11, pp. 2235-2248, 1999.

[82] L. F. Suhr, J. J. V. Olmos, C. Peucheret and I. T. Monroy, "Direct modulation and detection link using polybinary signaling," in OECC/ACOFT, Melbourne, 2014.

[83] J. J. V. Olmos, L. F. Suhr, B. Li and T. I. Monroy, "Five-level polybinary signaling for $10 \mathrm{Gbps}$ data transmission systems," Optics Express, vol. 21, no. 17, pp. 20417-20422, 2013.

[84] "IEEE P802.3bs 400 Gb/s Ethernet Task Force," 2018. [Online]. Available: http://www.ieee802.org/3/bs/. 
[85] N. Stojanovic, C. Prodaniuc, F. Karinou and Z. Qiang, "56-Gbit/s 4-D PAM-4 TCM transmission evaluation for 400-G data center applications," in OFC, Anaheim, 2016.

[86] L. Tao, Y. Ji, J. Liu, A. Pak, T. Lau, N. Chi and C. Lu, "Advanced modulation formats for short reach optical communication systems," IEEE Network, vol. 23, no. 6, pp. 6-13, 2013.

[87] P. J. Winzer and R.-J. Essiambre, "Advanced optical modulation formats," Proceedings of the IEEE, vol. 94, no. 5, pp. 952-985, 2006.

[88] J. Wei, N. Eiselt, H. Griesser, K. Grobe, M. Eiselt, J. J. V. Olmos, I. T. Monroy and J.-P. Elbers, "Demonstration of the first real-time end-to-end 40-Gb/s PAM-4 for next-generation access applications using 10-Gb/s transmitter," J. of Light. Tech., vol. 34, no. 7, pp. 1628-1635, 2016.

[89] X. Pang, O. Ozolins, S. Gaiarin, J. R. N. A. Kakkar, M. I. Olmedo, R. Schatz, A. Udalcovs, U. Westergren, D. Zibar, S. Popov and G. Jacobsen, "Experimental study of 1.55-um EML-based optical IM/DD PAM4/8 short reach systems," IEEE Photonics Tech. Letters, vol. 29, no. 6, pp. 523526, 2017.

[90] S. Walklin and J. Conradi, "On the relationship between chromatic dispersion and transmitter filter response in duobinary optical communication systems," IEEE Photonics Tech. Letters, vol. 9, no. 7, pp. 1005-1007, 1997.

[91] J. Wang and K. Petermann, "Small signal analysis for dispersive optical fiber communication systems," J. of Light. Tech. , vol. 10, no. 1, pp. 96-100, 1992.

[92] N. Alic, G. C. Papen, R. E. Saperstein, R. Jiang, C. Marki, Y. Fainman, S. Radic and P. A. Andrekson, "Experimental demonstration of $10 \mathrm{~Gb} / \mathrm{s}$ NRZ extended dispersion-limited reach over $600 \mathrm{~km}-\mathrm{SMF}$ link without optical dispersion compensation," in OFC, OWB7, 2006.

[93] F. Fresi, G. Meloni, M. Secondini, F. Cavaliere, L. Poti and E. Forestieri, "Short-reach distance extension through CAPS coding and DSP-free direct detection receiver," in ECOC, Th2.P2.SC3.10, Duesseldorf, 2016.

[94] E. Forestieri and G. Prati, "Novel optical line codes tolerant to fiber chromatic dispersion," J. of Light. Tech. , vol. 19, no. 11, pp. 1675-1684, 2001.

[95] D. Slepian and H. O. Pollak, "Prolate spheroidal wave functions, Fourier analysis and uncertainty I," The Bell System Tech. J., vol. 40, no. 1, pp. 43-63, 1961.

[96] H. J. Landau and H. O. Pollak, "Prolate spheroidal wave functions, Fourier analysis and uncertainty - II," The Bell System Tech. J., vol. 40, no. 1, pp. 65-84, 1961.

[97] [Online]. Available: http://www.ieee802.org/3/bs/public/15_01/index.shtml.

[98] J. L. Wei, J. D. Ingham, D. G. Cunningham, R. V. Penty and I. H. White, "Perfromance and power dissipation comparisons between $28 \mathrm{~Gb} / \mathrm{s}$ NRZ, PAM, CAP, and optical OFDM systems for data communication applications," J. of Light. Tech. , vol. 30, no. 20, pp. 3273-3280, 2012. 
[99] S. Bhoja, V. Parthasarathy and Z. Wang, "FEC Codes for 400 Gbps $802.3 \mathrm{bs,"} \mathrm{[Online].} \mathrm{Available:}$ http://www.ieee802.org/3/bs/public/14_11/parthasarathy_3bs_01a_1114.pdf.

[100] G. Ungerboeck, "Channel coding with multilevel/phase signals," IEEE Trans. on Information Theory, Vols. IT-28, no. 1, pp. 55-67, 1982.

[101] M. Hatamian, O. E. Agazzi, J. Creigh, H. Samueli, A. J. Castellano, D. Kruse, A. Madisetti, N. Yousefi, K. Bult, P. Pai, M. Wakayama, M. M. McConell and M. Colombatto, "Design considerations for gigabit Ethernet 1000BASE-T twisted pair transceivers," Proc. of the IEEE Custom Integrated Circuits Conf. , pp. 335-342, 1998.

[102] E. F. Haratsch and K. Azadet, "A 1-Gb/s joint equalizer and trellis decoder for 1000BASE-T gigabit Ethernet," IEEE J. of Solid-State Circuits, vol. 36, no. 3, pp. 374-384, 2001.

[103] "convolutional coding," [Online]. Available: http://web.mit.edu/6.02/www/f2010/handouts/lectures/L8.pdf.

[104] C. Langton, "Coding and decoding with convolutional codes," [Online]. Available: http://complextoreal.com/wp-content/uploads/2013/01/convo.pdf.

[105] X. Xu, E. Zhou, G. N. Liu, T. Zuo, Q. Zhong, L. Zhang, Y. Bao, X. Zhang, J. Li and Z. Li, "Advanced modulation formats for 400-Gbps short-reach optical inter-connection," Optics Express, vol. 23, p. 492, 2015.

[106] T. Tanaka, M. Nishihara, T. Takahara, W. Yan, L. Li, Z. Tao, M. Matsuda, K. Takabayashi and J. C. Rasmussen, "Experimental demonstration of 448-Gbps+ DMT transmission over 30-km SMF," in OFC, M2I.5, 2014.

[107] J. E. Kardontchik, "4D encoding in level-one's proposal for 1000BASE-T," Advanced Micro Devices, Sunnyvale, CA, 1997.

[108] N. Stojanovic, "Tail extrapolation in MLSE receivers using nonparametric channel model estimation," IEEE Trans. on Signal Processing, vol. 57, no. 1, 2009.

[109] G. Giunta and F. Benedetto, "A signal processing algorithm for multi-constant modulus equalization," in Int. Conf. on Telecommunications and Signal Processing, 2013.

[110] B. Sklar, "How I learned to love the trellis," IEEE Signal Processing Mag. , vol. 20 , no. 3, pp. 87102, 2003.

[111] J. Hanegauer and P. Hoeher, "A Viterbi algorithm with soft-decision outputs and its applications," Proc. GLOBECOM, vol. 3, pp. 1680-1686, 1989.

[112] [Online]. Available: http://100glambda.com/specifications/send/2-specifications/1-400g-fr4technical-spec-d1p0. 
[113] H. Mardoyan, M. A. Mestre, J. M. Estaran, F. Jorge, F. Blache, P. Angelini, A. Konczykowska, M. Riet, V. Nodjiadjim, J.-Y. Dupuy and S. Bigo, "84-, 100-, and 107-GBd PAM-4 intensity-modulation direct-detection transceiver for datacenter interconnects," J. of Light. Tech. , vol. 35, no. 6, pp. 1253-1259, 2017.

[114] X. Chen, C. Antonelli, S. Chandrasekhar, G. Raybon, J. Sinsky, A. Mecozzi, M. Shtaif and P. Winzer, "218-Gb/s single-wavelength, single-polarization, single-photodiode transmission over $125-\mathrm{km}$ of standard singlemode fiber using Kramers-Kronig detection," in OFC, Th5B.6, Los Angeles, 2017.

[115] H. Yamazaki, M. Nagatani, F. Hamaoka, S. Kanazawa, H. Nosaka, T. Hashimoto and Y. Miyamoto, "300-Gbps discrete multi-tone transmission using digital-preprocessed analog-multiplexed DAC with halved clock frequency and supressed image," in ECOC, Duesseldorf, 2016.

[116] [Online]. Available: http://micram.net/products/vega-application-systems/.

[117] L.-F. Wei, "Trellis-coded modulation with multidimensional constellation," IEEE Trans. on Information Theory, Vols. IT-33, no. 4, 1987.

[118] G. Ungerboeck, "Trellis-coded modulation with redundant signal set partitioning II: state of the art," IEEE Comm. Mag. , vol. 25, no. 2, 1987.

[119] R. Rios-Müller, J. Renaudier, M. A. Mestre and H. Mardoyan, "Multi-dimension coded PAM4 signalling for 100Gb/s short-reach transceivers," in OFC, Th1G.4, Duesseldorf, 2016.

[120] N. Stojanovic, F. Karinou, Z. Qiang and C. Prodaniuc, "Volterra and Wiener equalizers for shortreach 100G PAM-4 applications," J. of Light. Tech. , vol. 35, no. 21, 2017. 